Florida International University FIU Digital Commons

\title{
Comprehending Performance of Cross-Frames in Skewed Straight Steel I-Girder Bridges
}

Jawad H. Gull

jgull001@fiu.edu

DOI: $10.25148 /$ etd.FI14040810

Follow this and additional works at: https://digitalcommons.fiu.edu/etd

Part of the Civil Engineering Commons, Computational Engineering Commons, and the Structural Engineering Commons

\section{Recommended Citation}

Gull, Jawad H., "Comprehending Performance of Cross-Frames in Skewed Straight Steel I-Girder Bridges" (2014). FIU Electronic Theses and Dissertations. 1128.

https://digitalcommons.fiu.edu/etd/1128 


\section{FLORIDA INTERNATIONAL UNIVERSITY}

Miami, Florida

COMPREHENDING PERFORMANCE OF CROSS-FRAMES IN SKEWED

STRAIGHT STEEL I-GIRDER BRIDGES

A dissertation submitted in partial fulfillment of the

requirements for the degree of

DOCTOR OF PHILOSOPHY

in

CIVIL ENGINEERING

by

Jawad Hussain Gull

2014 
To: Dean Amir Mirmiran

College of Engineering and Computing

This dissertation, written by Jawad Hussain Gull, and entitled Comprehending Performance of Cross-frames in Skewed Straight Steel I-Girder Bridges, having been approved in respect to style and intellectual content, is referred to you for judgment.

We have read this dissertation and recommend that it be approved.

$\begin{array}{r}\text { Yimin Zhu } \\ \hline \text { Arindam Gan Chowdhury } \\ \hline \text { Ton-Lo Wang } \\ \hline \text { Amir Mirmiran } \\ \hline \text { Atorod Azizinamini, Major Professor }\end{array}$

Date of Defense: February 20, 2014

The dissertation of Jawad Hussain Gull is approved.

$\begin{array}{r}\text { Dean Amir Mirmiran } \\ \text { College of Engineering and Computing } \\ \hline \begin{array}{r}\text { Dean Lakshmi N. Reddi } \\ \text { University Graduate School }\end{array}\end{array}$

Florida International University, 2014 
(C) Copyright 2014 by Jawad Hussain Gull

All rights reserved. 


\section{DEDICATION}

I dedicate this dissertation to my daughter (Iman) and my wife (Maria) for loving, supporting and believing me, to my mother and father for giving me thinking beyond the conventional, to my brothers and sisters for admiring my achievements, and my adviser (Dr. Azizinamini) for his outstanding vision and guidance throughout the course of research. 


\section{ACKNOWLEDGMENTS}

First of all, I am thankful to the Divine Creator who has shown me the path that leads to success. He bestowed me with mercy, kinship, and contentment through difficult times.

This dissertation would not have been written without the love, affection, moral support, constant prays and encouragement of my wife, daughter, brothers, sisters, and all the rest of my family. My heartiest gratitude goes to my wife, Maria, for her love, patience, continuous encouragement, and the moral support.

I would like to recognize my adviser, Dr. Atorod Azizinamini, for his outstanding vision and intelligence in delineating the path of research. I really admire him for his down to earth approach in solving engineering problems, openness to new ideas, and involving graduate student in many research projects to broaden their understanding of the structural engineering.

I would like to thank Dr. Donald W. White and Dr. Todd Helwig for holding meetings, presentations and brainstorming sessions on framing of the skewed bridges. I am grateful to Dr. White for his time to have direct communications with me on my research. I would like to appreciate my dissertation committee members, Dr. Amir Mirmiran, Dr. Ton-Lo Wang, Dr. Arindam Gan Chowdhury, and Dr. Yimin Zhu for their constructive comments on my dissertation.

Special thanks to Dr. Yakel, for helping me by explaining theoretical concepts, technical details and efficient use of analysis software. Thanks to Corey Ginsberg from Center for Excellence in Writing for helping me with the technical write up. I would like 
to thank my fellow graduate students, Alireza Mohammadi, Huy Pham, Ramin Taghinezad for their friendship and collaboration.

I would like to thank Edgar Polo for helping me on the experimental work both in the laboratory and in the field. I would like to thank Haydee Caneda, Rachel Garcia and administrative staff at Florida International University for helping out with the departmental and university policies and procedures.

I would like to acknowledge the support of Florida Department of Transportation for providing the funding and holding technical discussions on various issues related to skewed bridges. I am thankful to Dennis Golabek, Jerry Hocking, Ben Goldsberry, Robert Robertson, and Sam Fallaha for their input. Special thanks to Dennis Golabek and Jerry Hocking for carrying out additional analysis to verify some of the recommendations presented in the dissertation. 


\title{
ABSTRACT OF THE DISSERTATION \\ COMPREHENDING PERFORMANCE OF CROSS-FRAMES IN SKEWED \\ STRAIGHT STEEL I-GIRDER BRIDGES
}

\author{
by
}

Jawad Hussain Gull

Florida International University, 2014

Miami, Florida

\section{Professor Atorod Azizinamini, Major Professor}

The effects of support in steel bridges can present significant challenges during the construction. The tendency of girders to twist or layovers during the construction can present a particularly challenging problem regarding detailing cross-frames that provide bracing to steel girders. Methods of detailing cross-frames have been investigated in the past to identify some of the issues related to the behavior of straight and skewed steel bridges. However, the absence of a complete and simplified design approach has led to disputes between stakeholders, costly repairs and delays in the construction.

The main objective of this research is to develop a complete and simplified design approach considering construction, fabrication and detailing of skewed bridges. This objective is achieved by comparing different detailing methods, understanding the mechanism by which skew effects develop in steel bridges, recommending simplified methods of analysis to evaluate them, and developing a complete and simplified design procedure for skew bridges.

Girder layovers, flange lateral bending stress, cross-frame forces, component of vertical deflections, component of vertical reactions and lateral reactions or lateral 
displacements are affected by detailing methods and are referred as lack-of-fit effects. The main conclusion of this research is that lack-of-fit effects for the Final Fit detailing method at the steel dead load stage are equal and opposite to the lack-of-fit effects for the Erected Fit detailing method at the total dead load stage. This conclusion has helped using 2D grid analyses for estimating these lack-of-fit effects for different detailing methods.

3D erection simulations are developed for estimating fit-up forces required to attach the cross-frames to girders. The maximum fit-up force estimated from the 2D grid analysis shows a reasonable agreement with the one obtained from the erection simulations. The erection sequence that reduces the maximum fit-up force is also found by erection simulations.

The line girder analysis is recommended for calculating cambers for the Final Fit detailing method. A combination of line girder analysis and 2D grid analysis is recommended for calculating cambers for the Erected Fit detailing method. Finally, flowcharts are developed that facilitate the selection of a detailing method and show the necessary design checks. 


\section{TABLE OF CONTENTS}

CHAPTER

PAGE

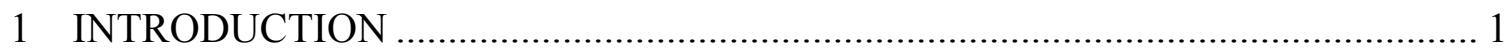

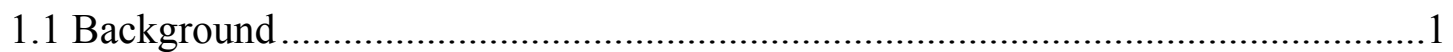

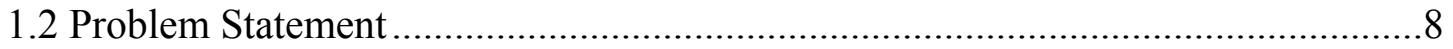

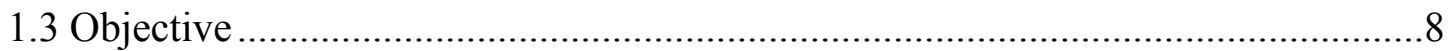

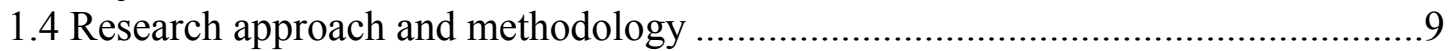

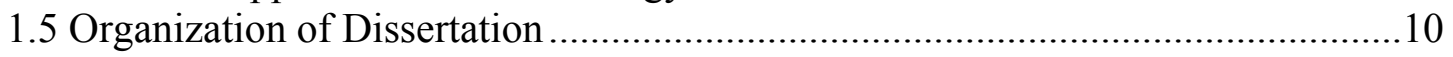

2 COMPARISON OF ERECTED FIT AND FINAL FIT DETAILING METHODS .. 13

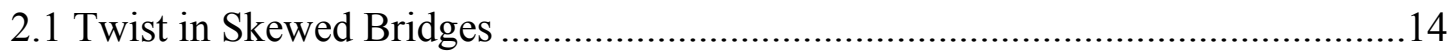

2.2 Existing and Proposed Terminologies ………………......................................16

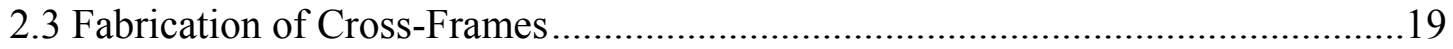

2.4 3D FEM analysis for Different Detailing Methods .............................................21

2.5 Structural Responses of the Skewed Bridges Affected By Different Detailing

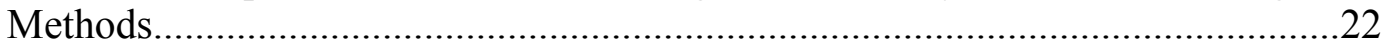

2.5.1 Girder Layovers....................................................................... 23

2.5.2 Vertical Deflections................................................................... 25

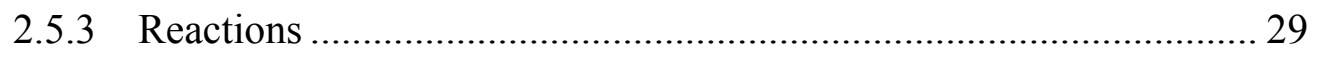

2.5.4 The Flange Lateral Bending Stress .................................................... 34

2.5.5 Cross-frame Forces........................................................................ 36

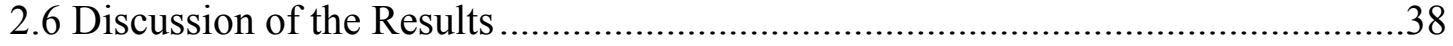

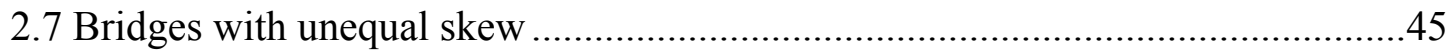

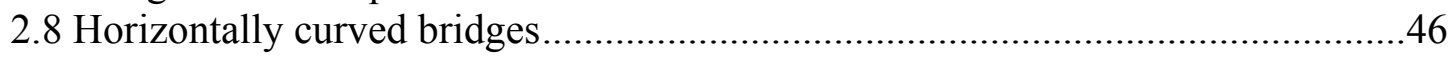

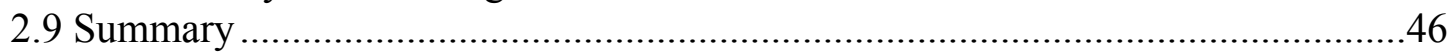

3 METHODS OF ANALYSIS FOR DIFFERENT DETAILING METHODS ............. 48

3.1 Method of analysis for the Erected Fit Detailing Method ………….......................48

3.1.1 1D Line Girder Analysis .............................................................. 48

3.1.2 2D Grid Analysis......................................................................... 49

3.1.3 3D FEM analysis .......................................................................... 51

3.2 Comparison of Different Methods of Analysis for the Erected Fit ........................51

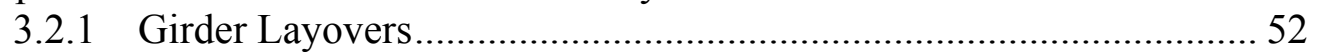

3.2.2 Component of the Vertical Deflections Due to the Lack-of-fit......... 55

3.2.3 Component of Vertical Reactions Due to Lack-of-fit ........................ 56



3.2.5 Cross-frame Forces......................................................................... 61

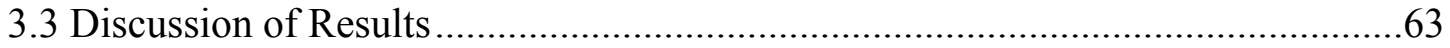

3.4 Methods of Analysis for the Final Fit Detailing Method........................................64

3.4.1 Reversing 2D GA Results for the Erected Fit ...................................... 65

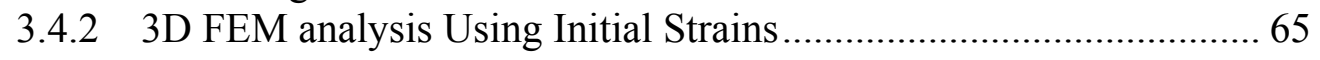

3.4.3 3D FEM analysis Using Dead and Live Cross-frames...................... 71

3.5 Comparison of Different Methods of Analysis for the Final Fit Detailing

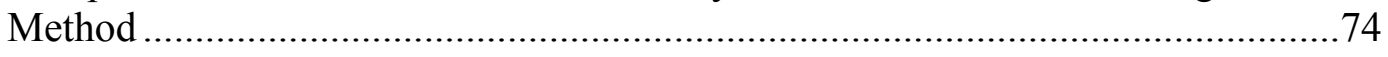




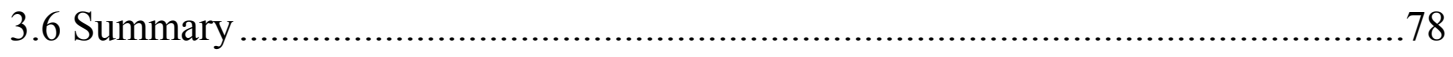

4 METHODS OF CALCULATING FIT-UP FORCES …………………………....... 80

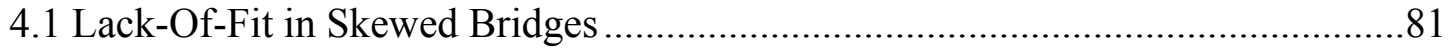

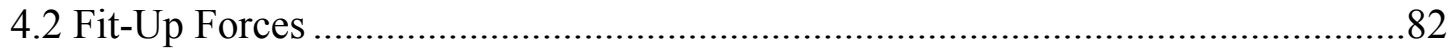

4.3 Proposed Methods of Calculating Fit-Up Forces....................................................8

4.3.1 Cross-frame Forces Method …………………................................. 84

4.3.2 3D Erection Simulation Method...................................................... 86

4.4 Discussion and Comparison of Fit-Up Forces ....................................................91

4.5 Effect of Different Practices on Fit-Up Forces .....................................................95

4.5.1 Different Erection Sequences ............................................................ 96

4.5.2 Distance of the First Intermediate Cross-frame from Support .......... 99

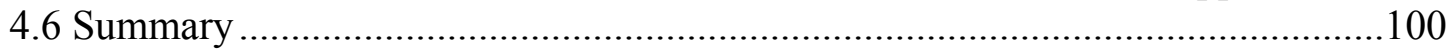

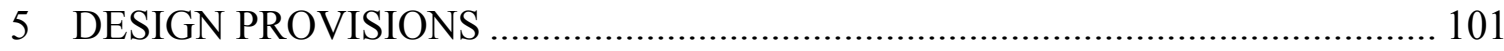

5.1 Recommendation on Calculation of Cambers ....................................................101

5.1.1 Verification of Recommendation Using Numerical Models............ 103

5.1.2 Summary and Discussion on Numerical Analysis .......................... 109

5.2 Structural Responses of the Skewed Bridges Affected By Detailing Methods ...110

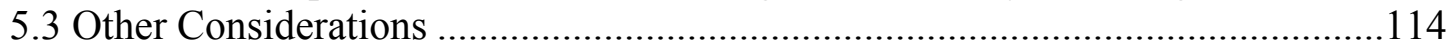

5.4 Parametric Studies .................................................................................

5.4.1 Effect of Cross-frame Stiffness …………………........................ 116

5.4.2 Effect of the Distance of the First Intermediate Cross-frame from Support ................................................................................ 118

5.4.3 Effect of Cross-frame Orientation ............................................... 122

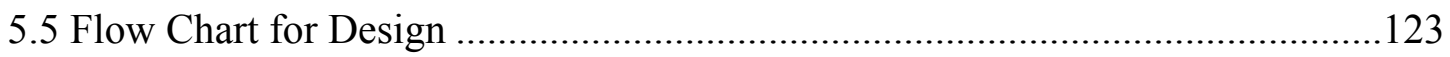

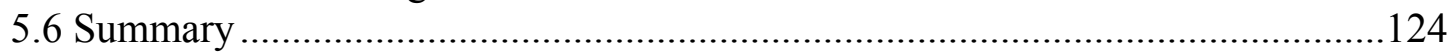

6 CONCLUSIONS AND RECOMMENDATIONS …………………..................... 126



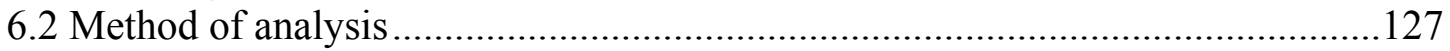

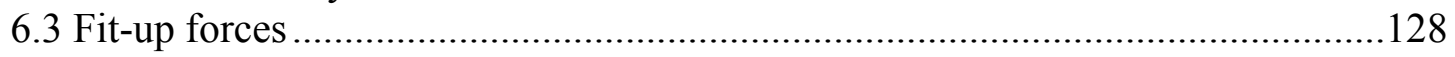

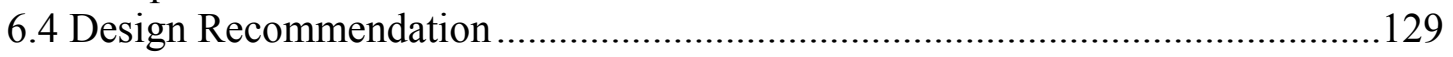



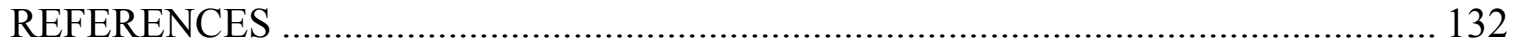

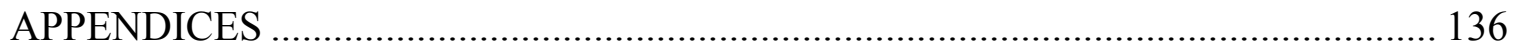




\section{LIST OF TABLES}

TABLE

PAGE

Table 4.1: Absolute Maximum Fit-Up force from different methods ............................94

Table 4.2: Absolute Maximum Fit-Up force from different erection sequences ............99

Table 5.1: Method of calculation of camber for different detailing methods ................103

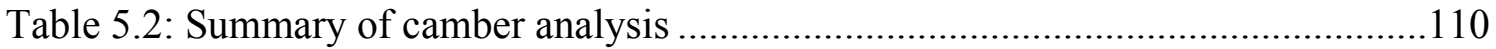

Table 5.3: Structural issues related to the Erected Fit and the Final Fit detailing

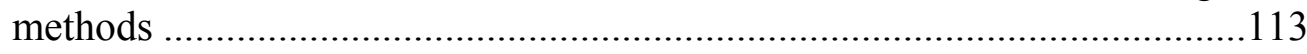

Table 5.4: Summary of effect area of cross-frame members on cross-frame forces......117 


\section{LIST OF FIGURES}

FIGURE

PAGE

Figure 2.1: Main sources of twist in straight skewed bridges.....................................16

Figure 2.2: The Erected Fit and the Final Fit detailing methods ...................................19

Figure 2.3: Differential camber in a skewed bridge .................................................20

Figure 2.4: Options for the Erected Fit detailing ...................................................21

Figure 2.5: The layovers in Girder 1 of Bridge A for different detailing methods at different loading stages .................................................................25

Figure 2.6: Vertical deflection in Girder 5 of Bridge A for different detailing methods at different loading stages.

Figure 2.7: Component of vertical deflection due lack-of-fit in Girder 5 of Bridge A for different detailing methods at different loading stages .....

Figure 2.8: Vertical reactions $\left(\mathrm{R}_{\mathrm{Y}}\right)$ of Bridge A for different detailing methods at different loading stages....

Figure 2.9: Component of vertical reaction due to lack-of-fit $\left(\mathrm{R}_{\mathrm{Y} 2}\right)$ for Bridge $\mathrm{A}$ for different detailing methods at different loading stages

Figure 2.10: Lateral reactions $\left(\mathrm{R}_{\mathrm{Z}}\right)$ of Bridge $\mathrm{A}$ for different detailing methods at different loading stages.

Figure 2.11: Flange lateral bending stress in top flange of Girder 1 of Bridge A for different detailing methods at different loading stages.

Figure 2.12: Cross-frame forces in top chord of cross-frames in Bay 7 of Bridge A for different detailing methods at different loading stages

Figure 2.13: Equal and opposite lack-of-fit

Figure 2.14: Cross-frame forces development mechanism explained in NCHRP 725

Figure 2.15: Cross-frame forces development mechanism.

Figure 3.1: Comparison of the layovers calculated by different analysis method for Girder 8 of Bridge A 
Figure 3.2: Comparison of the layovers calculated by different analysis method for Girder 8 of Bridge B...

Figure 3.3: Comparison of the $\mathrm{D}_{\mathrm{Y} 2}$ calculated by different analysis methods for Bridge A. .55

Figure 3.4: Comparison of the $\mathrm{D}_{\mathrm{Y} 2}$ calculated by different analysis methods for Bridge B.

Figure 3.5: Comparison of the $\mathrm{R}_{\mathrm{Y} 2}$ calculated by different analysis methods for Bridge A.

Figure 3.6: Comparison of $\mathrm{R}_{\mathrm{Y} 2}$ calculated by different analysis method for Bridge B-Erected Fit at the TDL stage

Figure 3.7: Comparison of flange lateral bending stress calculated by different analysis method in Girder 8 of Bridge A.

Figure 3.8: Comparison of flange lateral bending stress calculated by different analysis methods in Girder 4 of Bridge B

Figure 3.9: Comparison of cross-frame forces calculated by different analysis method for Bridge A.

Figure 3.10: Comparison of cross-frame forces calculated by different analysis method for Bridge B

Figure 3.11: Configurations to calculate initial strain in the cross-frames that are perpendicular to girder web

Figure 3.12: Configurations to calculate initial strain in the cross-frames that are parallel to skew

Figure 3.13: Application of concrete dead load on girders after killing cross-frame elements ....

Figure 3.14: Removal of concrete dead load from girders after making crossframe elements alive

Figure 3.15: Comparison of girder layovers calculated by different analysis methods

Figure 3.16: Comparison of the component of vertical deflection due to lack-of-fit $\left(D_{Y 2}\right)$ calculated by different analysis methods .76

Figure 3.17: Comparison of the vertical reactions due to lack-of-fit $\left(\mathrm{R}_{\mathrm{Y} 2}\right)$ calculated by different analysis methods.... 
Figure 3.18: Comparison of the flange lateral bending stress calculated by different analysis methods

Figure 3.19: Comparison of cross-frame forces calculated by different analysis methods .77

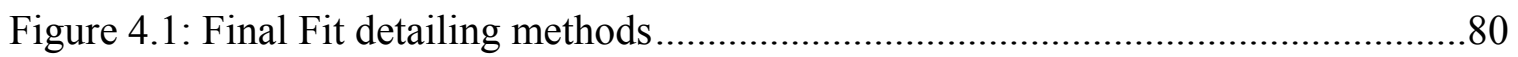

Figure 4.2: Differential camber in a skewed bridge .............................................. 82

Figure 4.3: Fit-up forces required to attach the cross-frames to the girders .....................83

Figure 4.4: Fit-up forces by resolving cross-frame forces at connection points...............86

Figure 4.5: Steps followed to calculate fit-up forces in erection simulation. ...................89

Figure 4.6: Geometry of Girder 4 of Bridge $\mathrm{C}$ after completion of erection....................90

Figure 4.7: Lateral Fit-up forces applied on Girder 3 of Bridge $\mathrm{C}$ for erecting cross-frames in Bay 3.

Figure 4.8: Vertical Fit-up forces applied on Girder 3 of Bridge $\mathrm{C}$ for erecting cross-frames in Bay 3

Figure 4.9: Cross-frame forces in $1^{\text {st }}$ cross-frame of Bay 1 of Bridge $\mathrm{C}$ during the erection.

Figure 4.10: Erection sequences for attaching cross-frames to girders

Figure 4.11: Fit-up force at the top of Girder 8 of Bridge A for erecting the crossframes in Bay 8

Figure 5.1: Verification of camber recommendation for the Erected Fit detailing method-Bridge A Girder 1

Figure 5.2: Verification of camber recommendation for the Final Fit detailing method-Bridge A Girder 1

Figure 5.3: Verification of camber recommendation for the Erected Fit detailing method-Bridge B Girder 1

Figure 5.4: Verification of camber recommendation for the Final Fit detailing method-Bridge B Girder 1

Figure 5.5: Verification of camber recommendation for the Erected Fit detailing method-Bridge C Girder 1 
Figure 5.6: Verification of camber recommendation for the Final Fit detailing method-Bridge C Girder 1

Figure 5.7: Effect of reducing the area of cross member on cross-frame forces in Bridge A-Erected Fit at the TDL stage.

Figure 5.8: Framing plans to study effects of the location of the first intermediate cross-frame from the support in Bridge A....

Figure 5.9: Variation of structural responses by changing the location of first intermediate cross-frame from the support in Bridge A- Erected Fit detailing under the TDL

Figure 5.10: Framing planes to study effects of location of $1^{\text {st }}$ intermediate crossframe from the support in a continuous bridge.

Figure 5.11: Variation of cross-frame forces by changing location of the first intermediate cross-frame from the support-Erected Fit at the TDL stage

Figure 5.12: Variation of vertical reactions by changing the location of the first intermediate cross-frame from the support-Erected Fit at the TDL stage

Figure 5.13: Different cross-frame orientations.

Figure 5.14: Layovers for different cross-frame orientations-Erected Fit at the TDL stage.

Figure 5.15: Flow chart to guide designer to deal with skewed bridges 124 


\section{ACRONYMS AND ABBREVIATIONS}

$\begin{array}{ll}\text { NL } & \text { No Load } \\ \text { TDL } & \text { Steel Dead Load } \\ \text { CDL } & \text { Total Dead Load } \\ \text { IGA } & \text { Concrete Dead Load } \\ \text { LGA } & \text { Line Girder Analysis } \\ \text { GA } & \text { Grid Analysis } \\ \text { 2D } & \text { Two dimensional } \\ \text { 3D } & \text { Three dimensional } \\ \text { FEM } & \text { Finite Element Method } \\ \text { SIP } & \text { Sit-In-Place } \\ \text { NCHRP } & \text { National Cooperative Highway Research Program } \\ \text { AASHTO } & \text { American Association of State Highway and Transportation Officials } \\ \text { CFs } & \text { Cross-frames }\end{array}$




\section{INTRODUCTION}

\subsection{Background}

In geometric design of roadways, there are a number of issues that require a roadway to overpass an obstruction at an angle other than ninety degrees. These issues include geometrical constraints of intersecting roadways, geological restrictions of the terrain surrounding the bridge, as well as other factors. Overpassing an obstruction at an angle other than ninety degrees requires construction of a skewed bridge having supports at an angle from the perpendicular to the longitudinal axis of the bridge. These supports that are not perpendicular to the longitudinal axis of the bridge are called skewed supports. Skewed supports in steel bridges lead to interactions between adjacent girders and the bracing that can result in a number of problems during the construction of skewed bridges.

One example of the construction issue is the construction of the skewed bridge in Wichita Falls, Texas. The cross slope after casting of deck was zero percent compared to six percent required by design. The problem delayed the project for more than one year and required two million US dollars to retrofit the bridge [1].

National Cooperative Highway Research Program (NCHRP) Synthesis 345 [2] summarizes some of the problems in construction of steel bridges, in particular, skewed and curved bridges. The synthesis states that erectors have reported the problems with regard to deflections, web plumpness, and tolerances to be applied to girder plumpness.

American Association of State Highway and Transportation Officials (AASHTO) (2012) [3]also recognized problems in construction of steel I-girder bridges as Article 
C6.7.2 states, "In some cases, failure to engineer the erection to achieve the intended final position of the girder, or to properly investigate potential outcome when detailing to achieve an intended final position of the girders, has resulted in construction delays and claims."

Numerical studies [4] on skewed bridges have identified flange lateral bending and accompanied flange lateral bending stress as one of the problems in skewed bridges. Compression flanges of bridges are discretely braced during the construction. AASHTO (2012) [3] article 6.10.1.6 states, "Lateral bending stresses in discretely braced flange shall be determined by structural analysis." To evaluate flange lateral bending stress, a refined analysis technique, such as finite element analysis, is required. This analysis is generally a very time-consuming task for design engineers.

Construction of the 63-degree skew simply supported bridge at Etna Interchange, S.R. 0028 shown tendency of the girders to move in lateral direction during the construction. Due to this lateral movement of the girders the bottom flanges move across the pot bearing and were no longer centered over the bearing [5]. Study of scaled model of a skewed bridge with girders made from poster board indicated girder movements due to rotations and differential deflection [6].

These problems discussed above are generally associated with the detailing methods used for detailing the cross-frames and girders [7] [8] [9] [10] [11] [12]. Detailing terminologies that are commonly used to describe the methods of detailing cross-frames and girders in steel bridges with skewed supports include No Load Fit (NLF), Steel Dead Load Fit (SDLF), Total Dead Load Fit (TDLF), consistent detailing, and inconsistent detailing [7] [8] [9] [10] [11] [12]. These terminologies generally refer to 
the plumb condition of the web at a particular loading stage (NLF, SDLF, and TDLF), or refer to the fact that girder and cross-frame might be detailed for the web to be plumb at different load stages (inconsistent detailing) or the same load stage (consistent detailing).

AASHTO (2012) [3] Article C6.7.2 describes two erected positions of I-girders in straight skewed and horizontally curved bridges. The girders can be erected as webs plum or webs out-of-plumb at three different loading stages. These loading stages are 1) No Load Stage 2) Steel Dead Load Stage 3) Total Dead Load Stage.

The use of these abstract terminologies described above has contributed to miscommunication between individuals in the bridge industry and has further led to the belief that skewed steel bridges are difficult to detail, design and construct. Therefore, there is a need to introduce simplified terminologies that are consistent with the field practice.

Different structural responses affected by detailing method, used for detailing cross-frames and girders, have been identified in the literature [7] [8] [9] [10] [11] [12]. These structural responses include vertical deflections, girder layovers, flange lateral bending stress, vertical reactions, and cross-frame forces. However, the girders in a skewed bridge also have tendency to move laterally on the support as indicated in the previous study [5] on the construction of skewed bridge. Therefore, there is a need to carry out additional three dimensional finite element method (3D FEM) analyses and identify the additional structural responses affected by detailing methods.

3D FEM analyses carried out for the skewed bridges detailed with different detailing methods also indicate the structural responses affected by different detailing methods have different magnitudes at different loading stages of construction [8] [9]. 
However, these studies do not establish a relationship of a response to a loading stage for different detailing methods. This relationship is important in identifying the mechanism by which responses related to different detailing methods are generated in the structure at different loading stages.

Most of the numerical studies carried out on detailing methods for skewed and horizontally curved bridges use 3D FEM analyses to determine the structural responses related to methods of detailing. These 3D FEM analyses are generally avoided in practice as much as possible. There are three main reasons to avoid 3D FEM analyses. 1) The level of effort and time required is high, 2) lack of consensus on importance of different details that can be included in the 3D FEM analyses and 3) lack of techniques to model certain details. For example, some studies [13] [14] emphasize modeling of Sit-In-Place (SIP) form into numerical models while others [8] do not include SIP in 3D FEM analyses. Similarly, there are different techniques available to model bearing pads in the bridges. The bearing pads can be modeled by torsional springs, compression-only struts, solid elements or layered shells.

Therefore, it is important to have simplified methods of analysis to be used in practice. These methods of analysis should be accurate enough to capture responses of the skewed bridges in relation to their detailing method.

There are different simplified methods of analysis the can be used to evaluate the responses of the skewed bridges due to the lack-of-fit of the cross-frames between the girders. One dimensional Line Grid Analysis (1D LGA) and two dimensional Grid Analysis (2D GA) are commonly used in practice. Prior studies have indicated that 1D 
LGA and 2D GA, not taking into account the warping stiffness of the girders, evaluate erroneous structural responses [15] [16].

The improved 2D GA introduced in the literature [7] [9]utilizes torsional constant's expression, derived in an earlier study [17], taking into account the warping stiffness. However, this improved 2D GA can be used for the bridges detailed with the No Load Fit detailing method. A concept of using 2D grid analysis for SDLF and TDLF detailing methods is given in NCHRP 725 [9]. This concept uses initial strains to model initial lack-of-fit between cross-frames and their connections to the girders, similar to what is used in 3D FEM analysis. Calculation of initial strains for every single crossframe member is cumbersome and error prone and therefore might not be used in practice. It is important to develop simplified 3D FEM analysis and 2D GA methods that can be used for all the detailing methods without involving tedious calculations.

Another important issue related to the TDLF detailing method is calculation of fitup force required to connect the cross-frames to girders during the erection of the steel bridge. The erection of the steel bridges was studied in the past. Analytical investigation of the erection sequence of the single span curved and skewed S.R. 2008 Ramp A-1 Bridge is presented in the literature [18]. This investigation monitors displacements, reactions, crane loads, and steel stresses during the erection of the skewed and curved bridges.

Field studies [19] on the erection of steel bridges has indicated that site constraints, methods of detailing cross-frames and the type of equipment used for the erection can have a significant influence on the erection of curved and skewed steel bridges. Some of these problems have been addressed by carrying out construction 
simulations [20] [21] [22]. These construction simulations however do not evaluate the effect of different sequence of attaching the cross frames to the girders on the fit-up forces.

These studies address some of the problems faced in the erection of the steel bridges, however, do not provide a method of estimating fit-up forces for the TDLF detailing method. The estimation of fit-up forces is important for making decisions regarding the selection of the detailing method and for making arrangements to apply the fit-up force if the TDLF is selected for detailing the bridge.

In addition to detailing methods, large stiffness of the cross-frame is also considered a contributing factor to the structural responses due to the lack-of-fit of crossframes between the girders in skewed bridges. Different configuration of the cross-frame, such as X-frame, K-frame, V-frame or Z-frames, can be used in framing a bridge. Each configuration can have different rolled shape, such as L-section, C-section, WT-section. Earlier tests on a single cross-frame of different configurations have shown that X-frame and K-frame have almost the same stiffness [23]. Also, Z-frame with single L-section has less stiffness, [24] but is not generally used in practice. X-type cross-frame using Lsections has less stiffness compared to the stiffness of the same cross-frame in a computer model using truss elements for the cross-frame's members. The reason for the smaller stiffness of X-type cross-frame using L-section compared to the stiffness X-type crossframe using other section is bending of L-section members due to eccentric connection [25].

The stiffness of the intermediate cross-frames can also be decreased by arranging them parallel to the skewed supports. One concern with arranging the intermediate cross- 
frame parallel to the skewed supports is the effectiveness of the brace point. Consequently, the stiffness requirement was developed for the cross-frames that are parallel to the skewed support [26]. Different connections for these skew cross-frames are also studied to develop a connection that is stiff enough to provide stability to the framing system [27].

Another concept to decrease the stiffness of the cross-frames is to use the lean-on bracing system [28] [29]. This concept has been implemented to a bridge with nearly a 60 degree skew support in Lubbock, Texas. Decreasing the stiffness of the cross-frames is good on the one hand, but can also result in large lateral displacements of bridge framing during casting of the concrete deck. There can be other framing options that do not rely on decreasing the stiffness of the cross-frames significantly and can be helpful in decreasing the responses of the skewed bridges affected by the detailing method. These framing options might be the distance of first intermediate cross-frame from the support, camber used for detailing the cross-frames, and the area of cross-frame members to be used in analysis. These options need to be evaluated to recommend design provisions.

AASHTO (2012) [3] Article 6.7.2 states, "Steel structures should be cambered during fabrication to compensate for dead load deflection and vertical alignment." The dead load vertical deflections in highly skewed bridges are complicated and depend on the method of detailing the cross-frames. Therefore, in order to camber the girders to meet AASHTO requirements, dead load vertical deflections need to be calculated correctly, taking into account the detailing method used for detailing the cross-frame.

Discussing different issues related to the framing of skewed bridges provides elements to develop a complete and simplified design approach that can be used in 
practice. Therefore, it is important to develop a coherent design approach by evaluating different framing options, structural responses associated with detailing methods, and methods of calculating cambers in skewed bridges.

\subsection{Problem Statement}

Lack of a simplified design approach considering the design, detailing, fabrication, erection and construction has resulted in claims, lawsuits, and other structural issues in the construction skewed bridges.

Following are the main question asked by the profession

- What is the effect of different detailing options on the construction of skewed bridges?

- What structural responses need to be checked to make sure that construction is safe and meets the requirements?

- What is the force required to fit the cross frames between their connection to girders?

- What method of analysis should be used for calculating cambers and what is the design procedure to be followed?

\subsection{Objective}

The main objective of this research is to comprehend the performance of cross frames in skewed bridges that can lead to simplified design approach for design, construction, fabrication and detailing the cross frames in straight skewed I-girder bridges.

This objective is achieved by completing the followings tasks: 
- Introduction of simplified terminologies consistent with field practices.

- Identification of structural responses affected by different detailing methods.

- Comparison of different detailing methods for straight skewed I girder bridges.

- Recommendation on the use of simplified methods of analysis for different detailing methods.

- Development of the erection simulation method based on three dimensional finite element method models simulating erection of a skewed bridge for the calculation of fit-up forces.

- Development of simplified methods for estimating the fit-up forces that can be used in the design office.

- Verification of simplified methods for calculating fit-up by the erection simulation method.

- Recommendation of method of analysis to be used for calculating camber for different detailing methods.

- Recommendation of specific structural responses that need to be considered in the design of skewed bridges.

- Comparison of different framing options available to designers for skewed bridges.

- Development of a simplified design flow chart for each detailing method.

\subsection{Research approach and methodology}

Structural responses affected by different detailing methods were identified by reviewing literature and carrying out 3D FEM analyses in ANSYS [28]. Different numerical and analytical analyses were conducted to understand the mechanism by which different structural responses develop in skewed bridges. Once the mechanism was understood, simplified methods of analysis are developed to come up with the simplified and practical design approach. The 2D grid analysis program is written in MATLAB [29] in order to have a tool for carrying out a simplified analysis for evaluation of structural 
responses in skewed bridges. Results obtained from the $2 \mathrm{D}$ grid analysis program are compared with the results obtained from the 3D FEM analysis to recommend a simplified method of analysis for the evaluation of structural responses in skewed bridges.

ANSYS Parametric Design Language (APDL) is used to develop the erection simulation for the estimating fit-up forces. Geometry updating capabilities of ANSYS has been utilized to update the bridge geometry after the erection of each cross-frame. The erection simulation not only provided a way of estimating the fit-up forces, but also showed how cross-frame forces changes during the erection of the skewed bridge detailed with the Final Fit detailing method.

Discussion of different issues related to the framing of skewed bridges, and development of simplified procedures to calculate the required responses, provided the elements required to develop a complete and simplified design approach that can be used in practice. Finally, flow charts and tables are developed to explain the design procedure and make recommendations on different design issues.

\subsection{Organization of Dissertation}

This dissertation is organized in six main chapters. Chapter 2 describes two detailing methods (Erected Fit, Final Fit) used for detailing the cross-frames in straight skewed I-Girder bridges. This chapter identifies different structural responses affected by different detailing methods at different construction stages of skewed bridges. Detailed discussion is provided on the relative importance and the magnitude for different loading stages for different detailing methods. It has been shown that these structural responses at different loading stages are affected primarily due to the lack-of-fit of the cross-frames 
between their connections to girders. This chapter concludes that lack-of-fit effects for the Final Fit detailing method at the Steel Dead Load stage are equal and opposite to the lack-of-fit effects for the Erected Fit detailing method at the Total Dead Load Stage.

Chapter 3 discusses different methods of analysis that can be used for calculating structural responses affected by different detailing methods. Different methods of analysis available in the literature are discussed and compared for both Erected Fit and Final Fit detailing methods. Two new methods of analysis are introduced for calculating lack-of-fit effects for the Final Fit detailing method at the Steel Dead Load stage. The new methods are very simple compared to the methods of analysis available in the literature for the Final Fit detailing method. Analysis results obtained from the new methods are compared to the available methods followed by a discussion explaining the reasons for agreement and differences. This chapter concludes that a single grid analysis can be used to calculate different responses affected by different detailing methods at different loading stages.

Chapter 4 discusses the fit-up forces that are required to fit the cross-frames, detailed with the Final Fit detailing method, between their connection points to the girders at the Steel Dead Load stage. This chapter introduces the 2D grid analysis and the 3D finite element method (FEM) to calculate the fit-up forces. 3D FEM attempts to mimic the erection of cross-frames following a particular erection sequence used in practice. The chapter provides the comparison of fit-up forces calculated from the 3D FEM and the 2D grid analysis method, followed by a discussion. The effect of different construction practices, such as, the erection sequence and the distance of the first intermediate cross-frame from the support, is evaluated. This chapter concludes that the 
simple 2D grid analysis can be used to estimate the fit-up forces. Erecting the cross-frame starting from the middle of a bay and moving toward the ends of the bay requires less maximum fit-up force compared to other erection sequences.

Chapter 5 discusses different design provisions for framing of straight skew Igirder bridges. Recommendations are made on the method of analysis for calculating the camber for different detailing methods. These recommendations are verified by carrying out the numerical analysis for different detailing methods at different loading stages. Different framing options, such as, the distance of the first intermediate cross-frame from the support, arranging cross-frames parallel to supports, and decrease in the stiffness of cross-frames, that a designer might consider for the framing a skewed bridge, are discussed. This chapter summarizes different structural responses due to the lack-of-fit of cross-frames between their connections to girders at different loading stages. Important structural responses related to the method of detailing are identified and a flow chart is developed to recommend a design procedure for each detailing method.

Chapter 6 provides the summary and conclusions of the research carried on the performance of cross-frames in the straight skewed I-girder bridges. 


\section{COMPARISON OF ERECTED FIT AND FINAL FIT DETAILING METHODS}

Earlier studies have reported a number of problems in the straight skewed steel bridges both during the girder erection and the placement of the concrete bridge deck. These problems include the excessive twist of the girders, uplift at the support locations, development of flange lateral bending stresses, and the difficulty in fitting the crossframes between their connections to girders during the erection [7] [8] [9] [31] [5]. These problems are generally associated with the detailing method used for the girders and cross-frames. Detailing terminologies that are commonly used for steel bridges with skewed supports include the No Load Fit (NLF), Steel Dead Load Fit (SDLF), Total Dead Load Fit (TDLF), consistent detailing, and inconsistent detailing[7] [8] [9][10] [11] [12]. These terminologies generally refer to the plumb condition of the web at a particular loading stage (NLF, SDLF, and TDLF) or refer to the fact that girder and cross-frames might be detailed for the web to be plumb at different loading stages (inconsistent detailing) or the same loading stage (consistent detailing). The use of these terminologies have contributed to the miscommunication between individuals in the bridge industry and have further led to the belief that the skewed steel bridges are difficult to detail, design and construct.

Different detailing methods have been investigated in the past to identify and compare the structural responses affected by the detailing methods. However, for the TDLF detailing method, 2D grid analysis cambers or 3D FEM cambers calculated from the vertical deflection of girders attached with cross-frames under the Total Dead Load, were used to simulate lack-of-fit effects [7] [8][9]. It should be noted that vertical 
deflection/camber obtained from the 2D GA or the 3D FEM analysis of girders attached with cross-frames are different from the deflection/camber obtained from the 1D Line Girder Analysis (LGA) or the Isolated Girder Analysis (IGA). The difference in camber can result in different lack-of-fit effects for SDLF and TDLF detailing methods. Therefore, it is important to understand the mechanism by which lack-of-fit effects develop in the skewed steel bridges.

The objectives of this chapter are to introduce of simplified terminologies consistent with field practices, identify the structural responses that are affected by different detailing methods, comparison of different detailing methods, and explaining the mechanism by which lack-of-fit effects develop in straight skewed I girder bridges. Before having detailed discussions on the particular issues described above, two primary sources of the twist in the skewed steel I-girder bridges are described in the following section.

\subsection{Twist in Skewed Bridges}

One of the contributing factors for the excessive girder twist in steel I-girder bridges with skewed supports is the relatively low torsional stiffness of the steel I-section during the construction. There are two main sources of the girder twist in straight skewed I-girder bridges: 1) the twist induced by the rotation of cross-frames parallel to skewed support 2) the twist induced by the differential girder vertical deflection that occurs at the two ends of cross-frames oriented perpendicular to girder web. Figure 2.1 shows the two different cross-frame orientations that can contribute to the girder twist. Figure 2.1(a) depicts a cross-frame parallel to skewed supports located at an exterior support of a 
skewed girder system. Flexure in the girders from gravity loads results in major axis bending that causes ends of the girders to rotate. Cross-frames attached to these ends rotate about their own axis that is parallel to skewed supports because the torsional stiffness of the cross-frames is very high compared to the torsional stiffness of the girders. This cross-frame rotation has a component parallel to the longitudinal axis of the girders resulting in twist of the girders. Figure 2.1(b) shows an intermediate cross-frame that is oriented perpendicular to girder webs and connects two adjacent skewed girders at different locations along the length of the individual girders. As the girder deflect from the applied dead load, the two ends of the cross-frame experience a differential vertical displacement. Since the in-plane stiffness of the cross-frames is very high compared to the torsional stiffness of the girders, differential vertical displacement at the ends of the cross-frame leads to torsional deformations in the girders.

The twist in the girders due to cross-frames is well understood. Previous studies have resulted in analytical expressions for both twist caused by the rotation of the crossframe at bearing line and twist caused by the differential deflection by assuming the cross-frames to be rigid [32] [8]. 


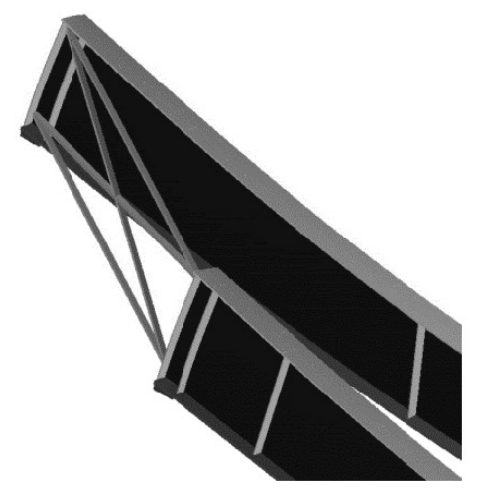

(a) Twist due to rotation of cross frames at bearing lines

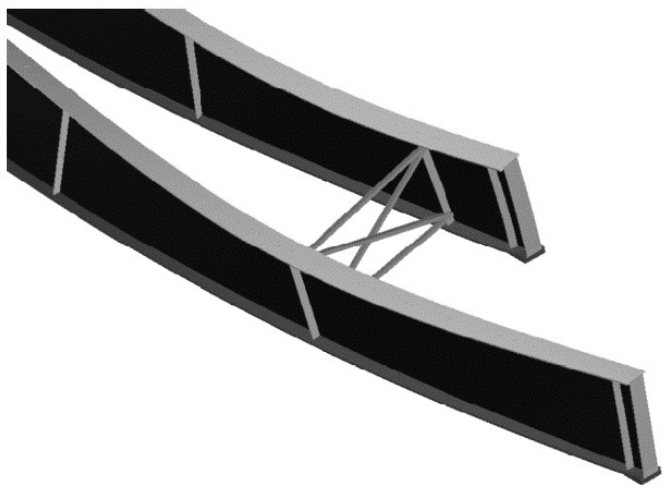

(b) Twist due to differential deflection

Figure 2.1: Main sources of twist in straight skewed bridges

\subsection{Existing and Proposed Terminologies}

The girder webs in straight skewed bridges can be detailed to be plumb at one of the different construction loading stages. As noted earlier, there are generally three stages that are used to reference when the girder webs are plumb: 1) the no load (NL) stage, 2) the steel dead load (SDL) stage, or 3) the total dead load (TDL) stage [33]. The definition of consistent detailing would be the case of both the girders and the crossframes to be detailed so that the webs are plumb at the same stage. The girders are often fabricated to be plumb at the NL stage, however cross-frames can be fabricated for web plumb at either NL or SDL or TDL stage. The term inconsistent detailing would be used to describe the situation where the girder webs are detailed to be plumb in one stage (usually the NL stage) and the cross-frames are detailed for the web to be plumb at a different stage (i.e. the SDL or TDL stages). Another set of terminologies, no load fit (NLF), steel dead load fit (SDLF), and total dead load fit (TDLF), is also used to describe above three scenarios. When the NLF method is employed, the cross-frames are 
fabricated for the web to be plumb at the NL stage. As the name implies, both the girder and cross-frame are detailed to fit when the girders rest on the ground in their fabricated NL geometry. However, once dead load is applied, the girder experiences twist due to bearing line rotation and differential deflection as explained earlier. When the SDLF method is employed, the cross-frames are fabricated for the web to be plumb at the SDL stage. In this scenario, both the girders and cross-frames are detailed to fit when the girders are erected and supported at the bearing lines (SDL stage). Similarly, when the TDLF method is employed, the cross-frames are fabricated for the web to be plumb at the TDL stage. In this scenario, both the girders and cross-frames are detailed to fit when the girders are supported at the bearing lines under the total construction dead load.

Attaching the cross frames to the girder require minimum effort or force for SDLF. This is because cross frames are generally attached to girder after placing the girders on support thereby girders deflected to the SDL condition that matches the crossframes detailing condition. If the NLF scenario is used, significant force may be necessary to attach the cross-frames to girders in case of un-shored or partially shored erection. If the TDLF scenario is used, significant force may be necessary to attach the cross-frames to girders. This is because at the time of the steel erection the girders are not deflected by the dead load from the concrete deck. As an example, consider the TDLF case in which the girders and cross-frames have been detailed for the web plumbness under the full construction dead load or TDL. In this case, cross-frames are detailed for out of plumb girder webs during the erection or SDL stage and that requires twisting of girders to attach the cross-frames. The amount of force necessary to attach the crossframes is highly dependent on the bridge geometry. 
The terms NLF, SDLF, and TDLF are generally idealized stages that may not actually occur in common practice. For example, in a typical steel bridge fabrication, using bolted field splices, the girders are fabricated for the NL stage (i.e. laydown). During the erection, holding cranes or temporary supports may be necessary to position the girders for installing cross-frame. Therefore, this stage is usually somewhere between the NL stage and SDL stage at the start of erection and gets close to the SDL stage near the completion of erection. As a result, the development of simplified terminologies that are consistent with the erection practices is desirable.

To reduce the miscommunication in this dissertation, the detailing terminologies the Erected Fit and the Final Fit are introduced in lieu of the NLF, SDLF, TDLF, consistent detailing, and inconsistent detailing.

In the Erected Fit detailing method, the cross-frames are detailed to fit between connections to girders at the erection or the SDL stage as shown in Figure 2.2 (a). These cross-frames do not fit between the connections to girders after the deck is casted or the TDL stage as shown in Figure 2.2 (a). In the Final Fit detailing method, the cross-frames are detailed to fit between the connections to girders after deck is casted or TDL stage as shown in Figure 2.2 (b). These cross-frames do not fit between the girders at the erection or the SDL stage as shown in Figure 2.2 (b). It is important to clarify here that Erected Fit is same as SDLF and Final Fit is same as TDLF for different analyses results shown in this dissertation.

Due to the lack-of-fit of cross-frames between the connections to girders, additional structural responses are developed in the skewed bridges. These structural responses, henceforth called lack-of-fit effects, are girder layovers, a component of 
vertical deflections, a component of reactions, flange lateral bending stresses, and crossframe forces.

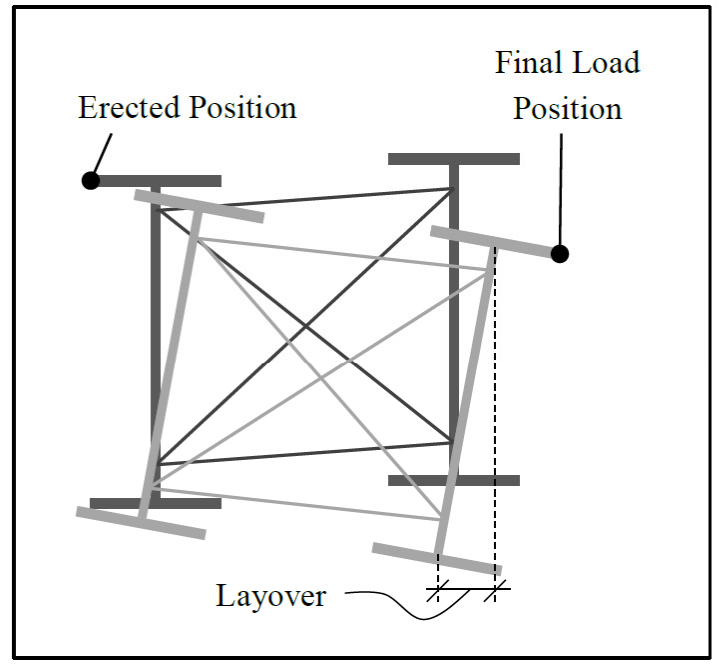

(a) Erected Fit detailing method

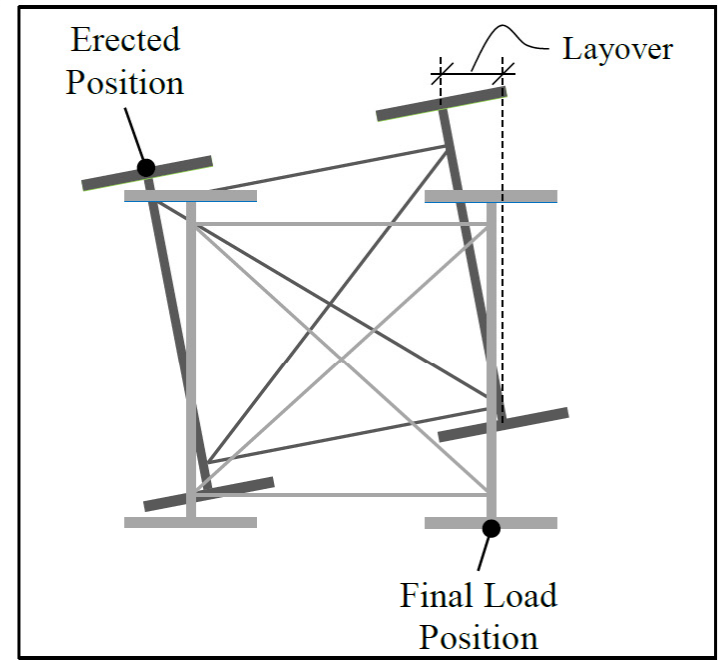

(b) Final Fit detailing method

\section{Figure 2.2: The Erected Fit and the Final Fit detailing methods}

\subsection{Fabrication of Cross-Frames}

Since cross-frames in skewed bridges are typically perpendicular to the girder web, the braces connect the two adjacent girders at different elevations due to the camber and the cross-slope. The difference in the elevation of girders due to the cross slope remains same at different loading stages and therefore does not contribute to lack-of-fit effects. However, the difference in girders' elevations due to the camber does contribute to lack-of-fit effects and is illustrated in Figure 2.3, which depicts the camber diagrams for three girders of a skewed bridge. The figure shows some of the detailing complexities that the differential camber produces with respect to cross-frame detailing. 


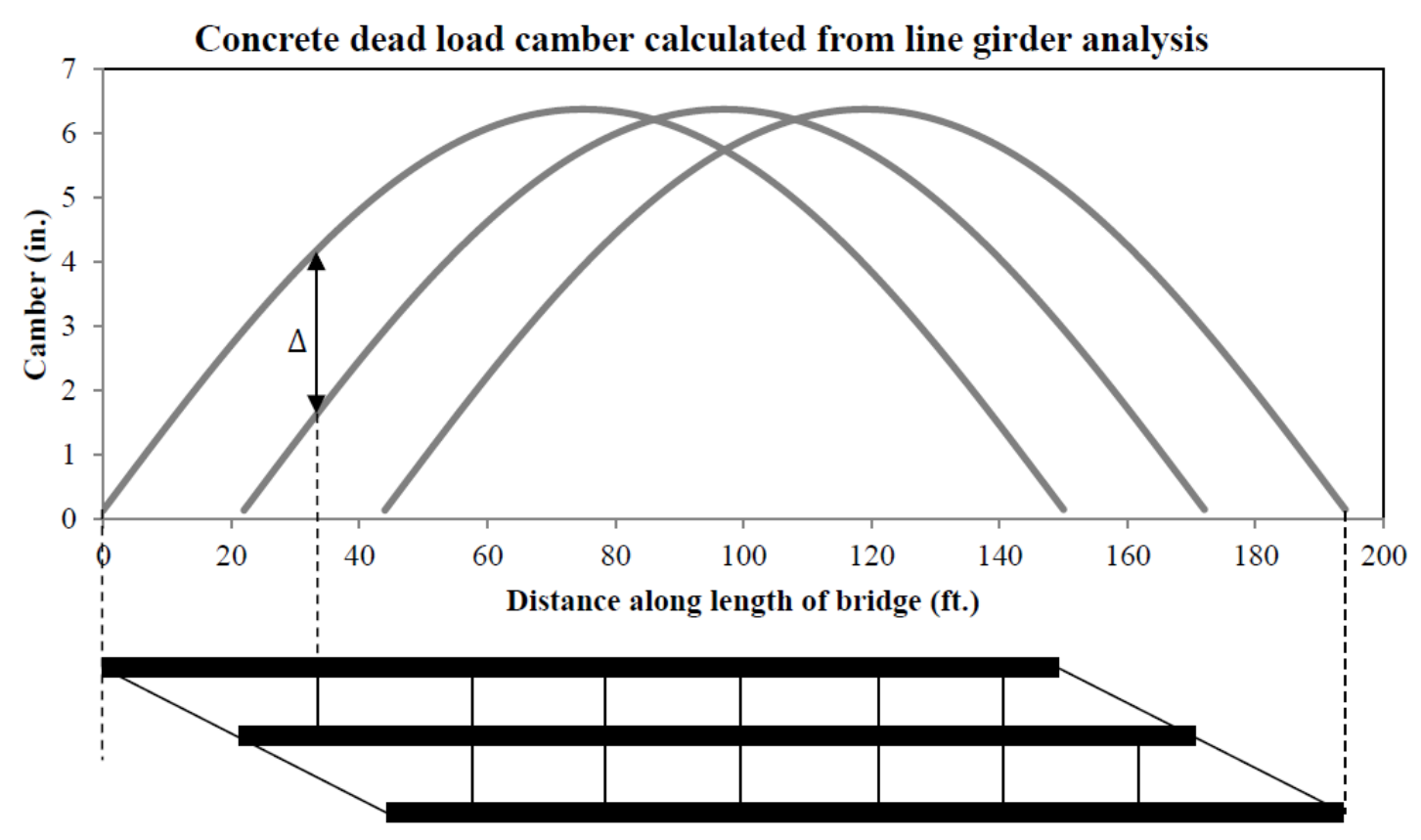

\section{Figure 2.3: Differential camber in a skewed bridge}

For the Erected Fit detailing method the cross-frames need to be fabricated taking into account the difference in in elevation of the girders due to camber $(\Delta)$. There are two approaches to fit the cross-frame to account for the differential camber or drop $(\Delta)$ :

a. Variable member lengths for each cross-frame as shown in Figure 2.4 (a).

b. The connection points of the cross-frames can be adjusted as shown in Figure 2.4 (b).

Option ' $a$ ' that is the variable member lengths for each cross-frame seems to have a lot of accompanied detailing and fabrication work. However, this option is generally used in the practice [34]because, the difference in elevation of girders due to the cross slope is built into the cross-frames regardless of the detailing methods used for the 
bridge. Therefore, automated jigs are developed that facilitate fabricating cross-frame members with different lengths for different drops.

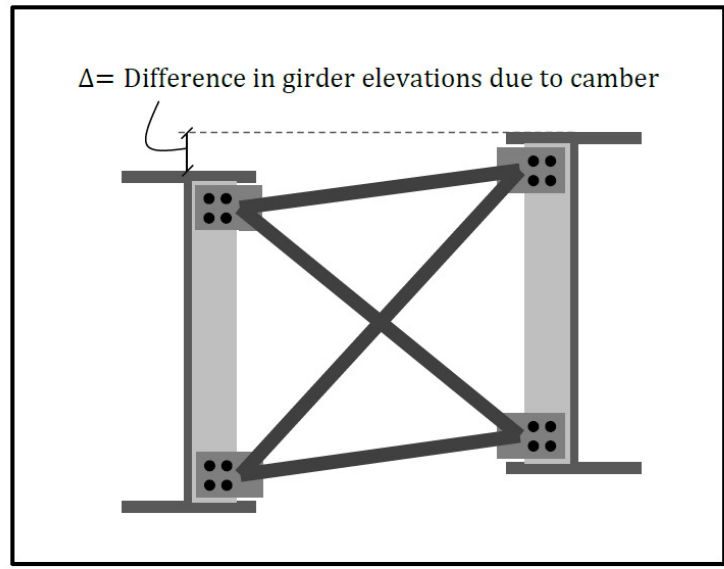

(a) Change lengths of cross frame members

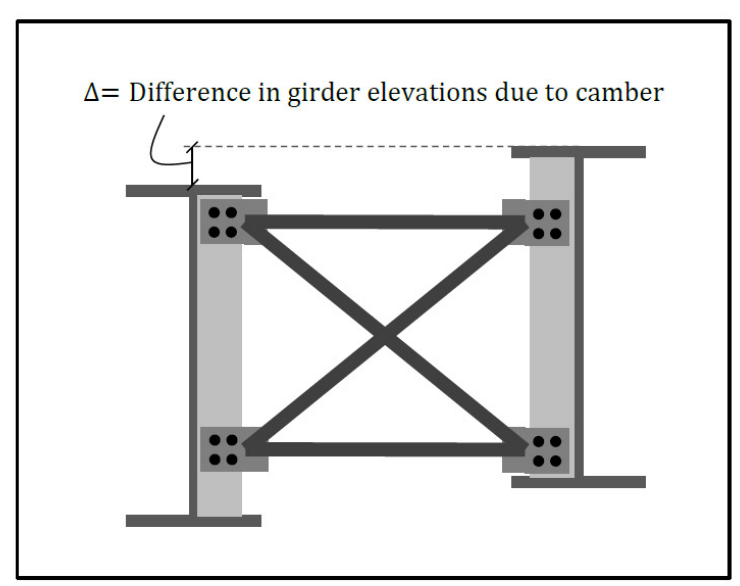

(b) Change location of connection of points

\section{Figure 2.4: Options for the Erected Fit detailing}

For the Final Fit detailing method cross-frames are fabricated to fit between their connections to girders at the TDL stage. The presence of the TDL removes the differential camber from the connection points. Therefore, cross-frames for the Final Fit detailing method are fabricated without taking into account the TDL cambers.

\subsection{D FEM analysis for Different Detailing Methods}

During the conduct of research, numerous three dimensional analyses were carried out using ANSYS [29]. Three dimensional Finite Element Method (3D FEM) analyses can be used with different modeling techniques. For example, in a 3D FEM analysis the flanges can be modeled using either beam elements or shell elements with or without bearing pads. Results presented in this dissertation are from 3D FEM analyses having flanges modeled by shell elements. These models also include bearing pads that are modeled by solid elements having the modulus of elasticity of $10 \mathrm{ksi}$. 
The 3D FEM analysis for the Erected Fit detailing method can be accomplished by applying the SDL on girders without attaching cross-frames. Once the SDL is applied, cross-frames are attached to the girders followed by the application of the concrete dead load (CDL).

The 3D FEM analysis for the Final Fit detailing method can be carried using two different approaches. The first approach uses imposing initial strains in cross-frame members. Carrying out the 3D FEM analysis by using initial strain approach [8] requires the following steps:

Step 1. Build the entire model of the bridge steel frame having girders attached together with cross-frames.

Step 2. Impose initial strain in the cross-frame members to simulate the lack-of-fit between cross-frames and girders at the SDL stage (initial strains are calculated from the camber diagram see section 3.4.2 for details).

Step 3. Apply the load simulating the wet concrete weight.

The second approach relies on applying the concrete dead load followed by activating the cross-frame members followed by removing the concrete dead load. Detail of this approach is provided in section 3.4.3. The results presented in this chapter are obtained from the initial strain approach.

\subsection{Structural Responses of the Skewed Bridges Affected By Different Detailing Methods}

Different structural responses affected by methods of detailing the cross-frames are identified from the literature review and conducting 3D FEM analyses. The structural 
responses that are affected by lack-of-fit of cross-frames between their connections girders include girder layovers, deflections, reactions, flange lateral bending stress, and cross-frame forces. The components of these structural responses affected by the lack-offit henceforth called lack-of-fit effects. These lack-of-fit effects appear after attaching the cross-frames to girders at a loading stage at which cross-frames do not fit between their connections to girders. Therefore, these effects appear at the TDL stage for the Erected Fit detailing method and at the SDL stage for the Final Fit detailing method.

Four bridges that are Bridge A, Bridge B, Bridge $\mathrm{B} 2$ and Bridge $\mathrm{C}$, were used to compare the lack-of-fit effects for the Final Fit and the Erected Fit detailing methods at different loading stages. 3D FEM analyses of all three bridges yielded similar comparisons; therefore, the results obtained from Bridge A are used in section 2.5.1 through 2.5.5. The results obtained for Bridge B, Bridge B2 and Bridge $\mathrm{C}$ are shown in Appendix B.

Bridge A is an extreme case of straight skewed bridges and is used to show extreme skew effects in previous studies [1] [2] [3]. Bridge A has $300 \mathrm{ft}$. long, 144 inches deep girders simply supported on $70.4^{\circ}$ skewed supports. The girders of Bridge A are braced with X-type cross-frames containing L6 x $6 \times 1$ angles. The bridge uses staggered cross-frames between 9 girders that are spaced at $9.25 \mathrm{ft}$. c/c spacing. The detailed framing plan and girder sizes are shown in Appendix A.

\subsubsection{Girder Layovers}

The girder layovers are defined as the lateral displacement from the center of top flange to the center of bottom flange at any particular section of the girder. As long as the 
load levels are less than a small fraction of the critical elastic buckling load at the factored strength load levels, girder layovers are not of any structural consequence, i.e., they do not have any significant impact on the strength of the structural system [36]. The NCHRP 725 report [9] recommends that when the factored loads under the appropriate strength load combinations are less than approximately $10 \%$ of the estimated elastic buckling load level, global second-order amplification can be neglected in the strength checks. In addition, AASHTO [3] Article 6.10.1.6 currently allows the engineer to neglect local amplification of flange lateral bending stresses between cross-frame locations when the factored loads are less than $15 \%$ of the estimated elastic lateraltorsional bucking load for a given girder unbraced length. Both of the above limits are based on judgment. If these limits are satisfied at factored load levels, or if they are not satisfied but second-order amplification is addressed in the calculation of the factored strength load requirements, then the impact of any dead load girder layovers on the strength of the system is negligible.

However, it is recommended that girder layovers should be calculated at the relevant loading stage of the construction and be communicated to the parties involved (owner, fabricators, contractors and erector) in the construction of skewed bridges. For the Erected Fit, girder layovers appear after casting of the deck. For the Final Fit, girder layovers appear at the SDL stage after attaching the cross-frames to girders.

The girder layovers, along the length of Girder 1 of Bridge A, are obtained from the 3D FEM analysis for different detailing methods at different loading stages of construction and are shown in Figure 2.5. The following observations can be noted by inspecting the data presented in Figure 2.5 for the Final Fit detailing method: 
- Girder layovers are zero at the TDL stage because for the Final Fit detailing method cross-frames are fabricated to fit between their connections to girders and there is no lack-of-fit between the cross-frames and their connections to girders.

- Girder layovers are not zero at the SDL stage because for the Final Fit detailing method cross-frames do not fit between their connections to girders at the SDL stage. Therefore, when cross-frames are connected to girders, girder layovers appears in the girders due to the lack-of-fit.

Reverse is true for the Erected Fit detailing method. Also notice that girder layovers for the Final Fit at the SDL stage are equal and opposite to girder layovers for the Erected Fit at the TDL stage. Similar observations were observed for other bridges analyzed as part of this study. Results for these bridges are shown in Appendix B.

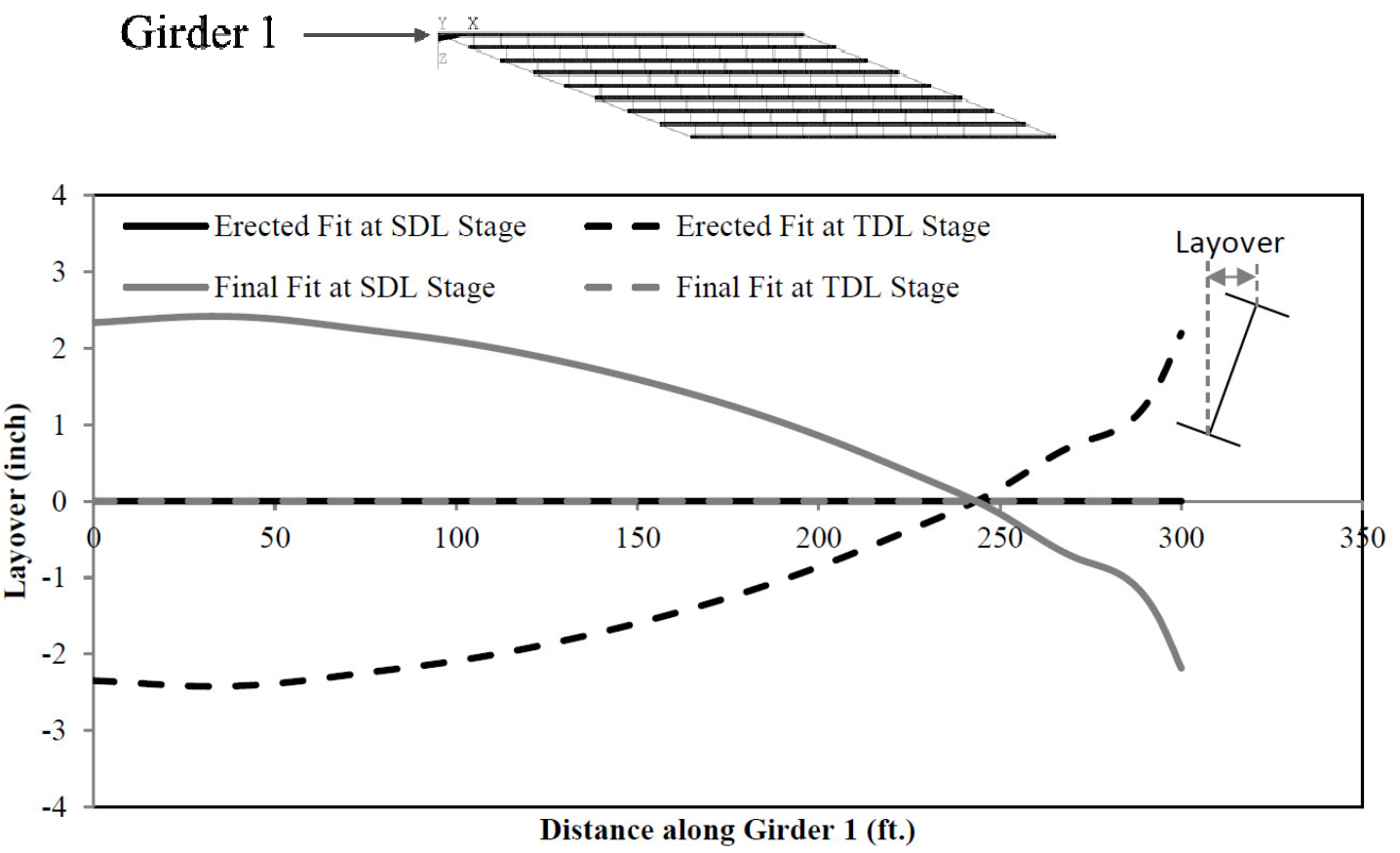

Figure 2.5: Girder layovers in Girder 1 of Bridge A for different detailing methods at different loading stages

\subsubsection{Vertical Deflections}

In straight skew I-girder bridges, the vertical deflections of the girders are affected by the lack-of-fit of cross-frames between their connections to girders. The 
effect on vertical deflections might be temporary or permanent depending on the method of detailing. Therefore, it is important to calculate vertical deflections taking into account the detailing method and camber the girders accordingly.

Vertical deflections for Bridge A are obtained from the 3D FEM analysis for different detailing methods at different loading stages of the construction and are shown in Figure 2.6. As mentioned earlier that vertical deflections of girders at a particular loading stage may or may not be affected by the lack-of-fit of cross-frames between their connections to girders depending on the detailing method used. Therefore, the vertical deflections obtained from isolated girder analysis (IGA) are also plotted in Figure 2.6 to make clear comparison. In IGA, dead loads are applied to isolated girders not connected to each other with the cross-frames. Therefore, IGA deflections do not include any vertical deflections due to the lack-of-fit of cross-frames to their connections to girders.

Following observations can be made by inspecting data presented in Figure 2.6:

- For the Final Fit detailing method at the TDL stage vertical deflections are in good agreement with vertical deflections from the IGA for the TDL. Therefore, it can be argued that vertical deflections for the Final Fit detailing method are not affected by the lack-of-fit at the TDL stage.

- Vertical deflections for the Final Fit detailing method at the SDL stage are not in a good match with the vertical deflections obtained from IGA for the SDL. Therefore, vertical deflections are affected by the lack-of-fit for the Final Fit detailing method at the SDL stage.

Reverse is true for the Erected Fit detailing method. Similar results were obtained for other bridges analyzed as a part of this study. 


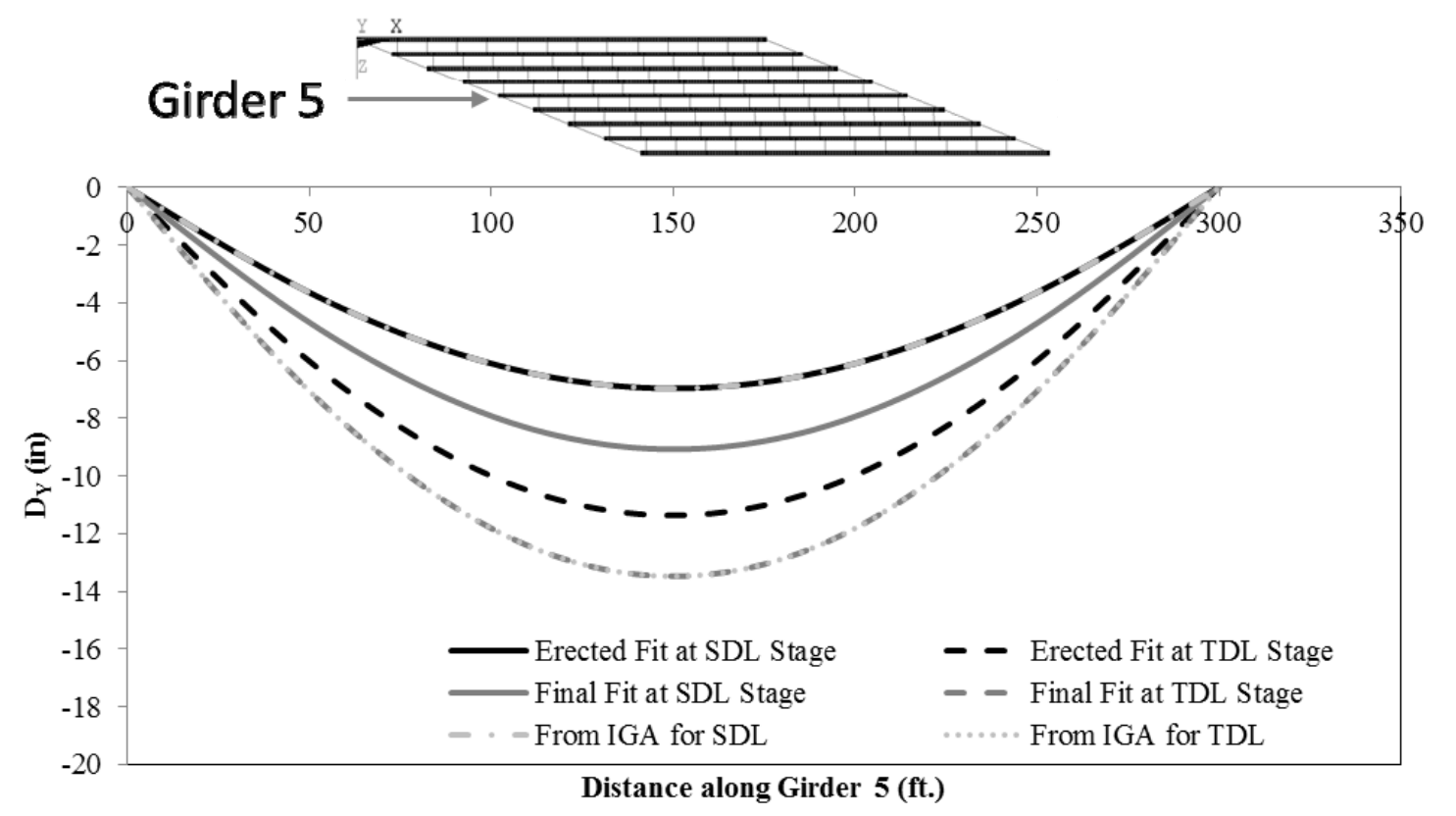

Figure 2.6: Vertical deflection in Girder 5 of Bridge A for different detailing methods at different loading stages

Vertical deflections are divided into two components in order to further distinguish the component of vertical deflections due to the lack-of-fit. The vertical deflection $\left(\mathrm{D}_{\mathrm{Y}}\right)$ at a particular loading stage can be divided into two components as shown by the following equation.

$$
\mathrm{D}_{\mathrm{Y}}=\mathrm{D}_{\mathrm{Y} 1}+\mathrm{D}_{\mathrm{Y} 2}
$$

$\mathrm{D}_{\mathrm{Y} 1}$ is the component of vertical deflections from the dead load and can be estimated from Isolated Girder Analysis (IGA) without attaching the cross-frames. $\mathrm{D}_{\mathrm{Y} 2}$ is the component of vertical deflections due to the lack-of-fit and can be estimated by rearranging Eq. (2.1) as follows:

$$
\mathrm{D}_{\mathrm{Y} 2}=\mathrm{D}_{\mathrm{Y}}-\mathrm{D}_{\mathrm{Y} 1}
$$


Component of the vertical deflection due to lack-of-fit $\left(\mathrm{D}_{\mathrm{Y} 2}\right)$ is obtained for different detailing methods at different loading stages and plotted in Figure 2.7.

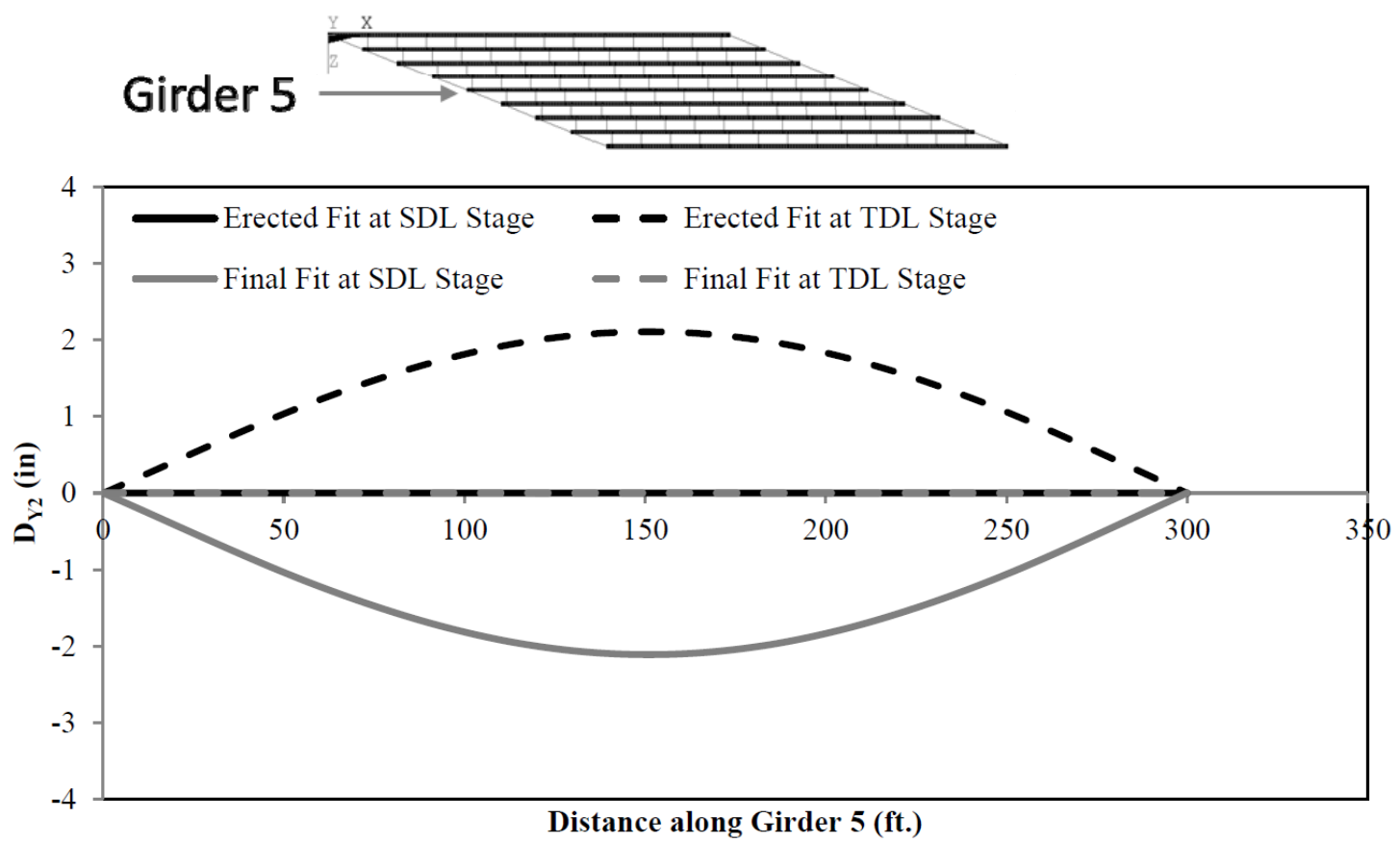

Figure 2.7: Component of the vertical deflection due lack-of-fit in Girder 5 of Bridge A for different detailing methods at different loading stages

Following observations can be made by inspecting the data presented in Figure 2.7:

- For the Final Fit detailing method, $\mathrm{D}_{\mathrm{Y} 2}$ is zero at the TDL stage because for the Final Fit detailing method cross-frames are detailed to fit between their connections to girders and there is no lack-of-fit.

- For the Final Fit detailing method, $\mathrm{D}_{\mathrm{Y} 2}$ is not zero at the SDL stage because for the Final Fit detailing method cross-frames do not fit between their connections to girders at the SDL stage.

Reverse is true for the Erected Fit detailing method. Also notice that $\mathrm{D}_{\mathrm{Y} 2}$ for the Final Fit at the SDL stage is equal and opposite to $\mathrm{D}_{\mathrm{Y} 2}$ for the Erected Fit at the TDL 
stage. Similar observations were observed for other bridges analyzed as part of this study. These results are shown in Appendix B.

\subsubsection{Reactions}

In straight skewed I-girder bridges, the girders can have negative vertical reactions resulting in lifting of girders from supports after the erection is complete or after the casting of concrete deck depending on the detailing method used. Therefore, it is important to check vertical reactions for straight skewed bridges in order to know chances of lift up.

Vertical reactions for Bridge A are obtained from the 3D FEM analysis for different detailing methods at different loading stages of the construction and are shown in Figure 2.8. Similar to vertical deflections, vertical reactions of girders at a particular loading stage may or may not be affected by the lack-of-fit of cross-frames depending on the detailing method used. Therefore, vertical reactions obtained from isolated girder analysis (IGA) are also plotted in Figure 2.8 to make clear comparison. As described earlier, in IGA, dead loads are applied to isolated girders not connected to each other with the cross-frames. Therefore, vertical reactions obtained from IGA do not include any vertical reactions due to the lack-of-fit of cross-frames.

Following observations can be made by inspecting data presented in Figure 2.8:

- For the Final Fit detailing method at the TDL stage, vertical reactions are in good agreement with vertical reactions from the IGA for the TDL. Therefore, it can be argued that vertical reactions for the Final Fit detailing method are not affected by the lack-of-fit at the TDL stage.

- Vertical reactions for the Final Fit detailing method at the SDL stage are not in a good match with the vertical reactions obtained from IGA for the SDL. Therefore, 
the vertical reactions are affected by the lack-of-fit for the Final Fit detailing method at the SDL stage.

Reverse is true for the Erected Fit detailing method. Similar results were obtained for other bridges analyzed as a part of this study.

Further, investigating Figure 2.8 reveals that for Bridge A negative vertical reactions can be seen at obtuse corners of the bridge (Support 1 of Girder 9 and Support 2 of Girder 1) for the Final Fit detailing method at the SDL stage. However, the SDL stage is a transient stage in the construction of skewed bridges. Therefore, such negative vertical reactions causing the girders to lift up from the support are temporary and are not problematic. The girders are seated on the supports once concrete deck is placed, as shown in Figure 2.8 for the Final Fit detailing method at the TDL stage (all vertical reactions are positive and uniform). Therefore, one only need to check for the uplift at the TDL stage for the Erected Fit detailing method, because any negative vertical reaction at the TDL stage is permanent. 


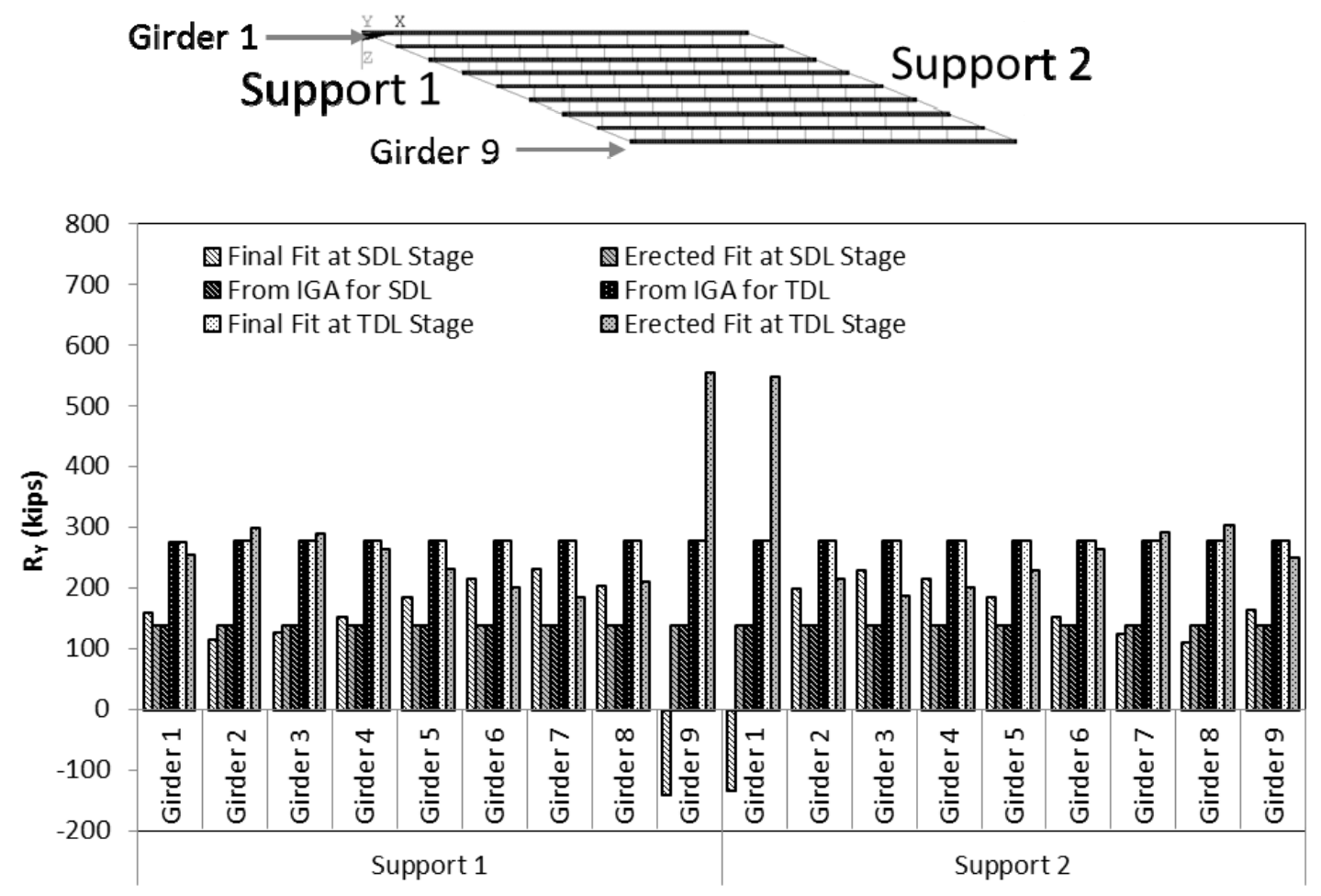

Figure 2.8: Vertical reactions $\left(R_{Y}\right)$ of Bridge $A$ for different detailing methods at different loading stages

In order to further distinguish the component of vertical reactions due to the lackof-fit, vertical reactions are divided into two components. A vertical reaction at a particular loading stage consists of two components as shown by the following equation

$$
\mathrm{R}_{\mathrm{Y}}=\mathrm{R}_{\mathrm{Y} 1}+\mathrm{R}_{\mathrm{Y} 2}
$$

$\mathrm{R}_{\mathrm{Y} 1}$ is component of the vertical reaction from dead load and can be estimated from isolated girder analysis (IGA). $\mathrm{R}_{\mathrm{Y} 2}$ is the component of vertical reaction due to lackof-fit and can be estimated by rearranging Eq. (2.2) as follows

$$
\mathrm{R}_{\mathrm{Y} 2}=\mathrm{R}_{\mathrm{Y}}-\mathrm{R}_{\mathrm{Y} 1}
$$


The component of the vertical reaction due to the lack-of-fit $\left(\mathrm{R}_{\mathrm{Y} 1}\right)$ is obtained for different detailing methods at different loading stages and plotted in Figure 2.9.
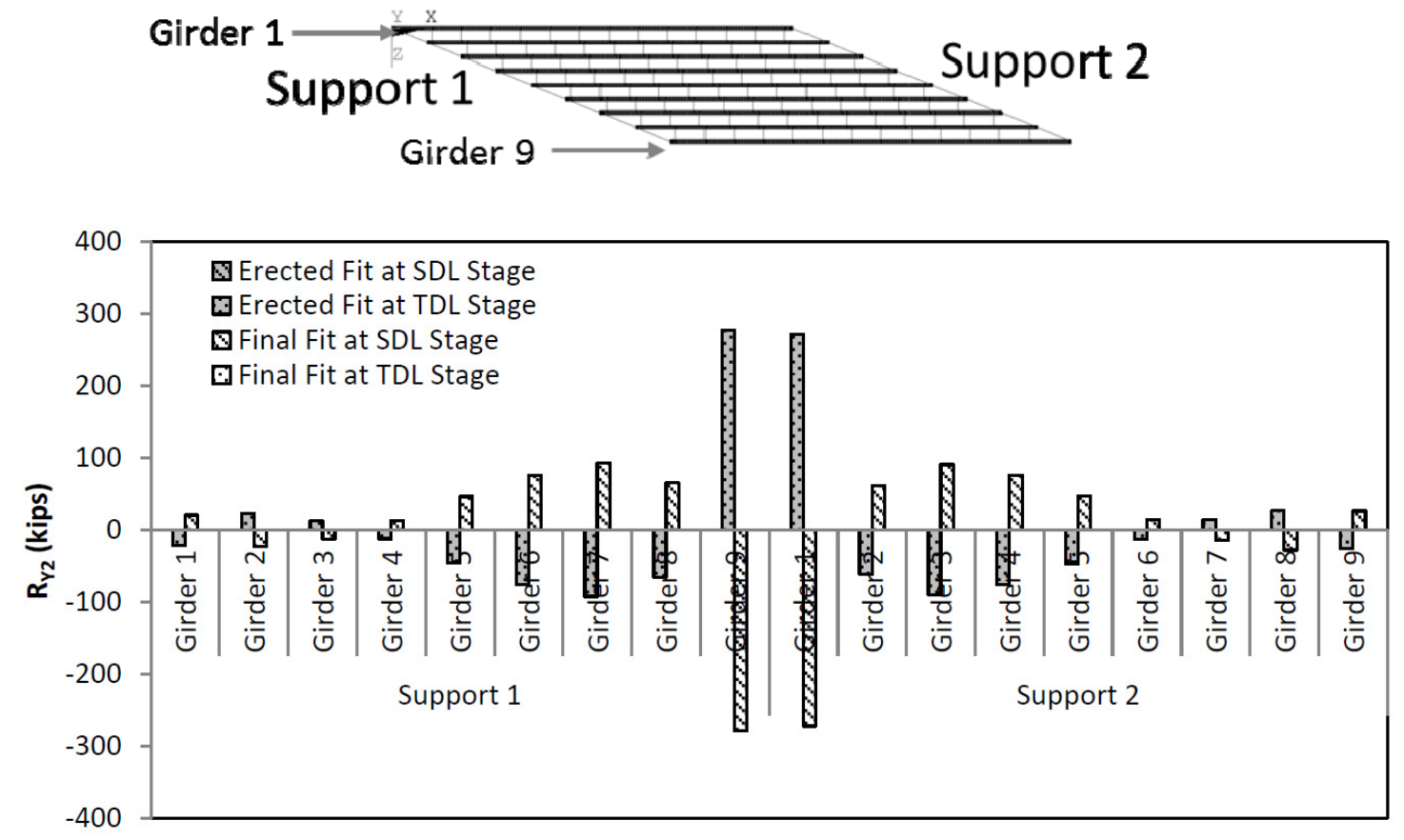

Figure 2.9: Component of vertical reaction due to lack-of-fit $\left(R_{Y 2}\right)$ for Bridge $A$ for different detailing methods at different loading stages

Following observations can be made by inspecting data presented in Figure 2.9:

- For the Final Fit detailing method, $\mathrm{R}_{\mathrm{Y} 2}$ is zero at the TDL stage because for the Final Fit detailing method cross-frames are fabricated to fit between their connections to girders and there is no lack-of-fit.

- For the Final Fit detailing method, $\mathrm{R}_{\mathrm{Y} 2}$ is not zero at the SDL stage because for the Final Fit detailing method cross-frames do not fit between the girders at the SDL stage.

Reverse is true for the Erected Fit detailing method. Also notice that the $\mathrm{R}_{\mathrm{Y} 2}$ for the Final Fit at the SDL stage is equal and opposite to $\mathrm{R}_{\mathrm{Y} 2}$ for the Erected Fit at the TDL 
stage. Similar observations were observed for other bridges analyzed as a part of this study.

Twisting of girders at supports causes bottom flanges to move across the bearings. This lateral movement of bottom flanges has been mentioned earlier in a study [5]on construction of the 63-degree skew simply supported bridge at Etna Interchange. If this lateral movement of bottom flanges is restrained by guided bearings or any other means, lateral reactions develop at constraints. Magnitude of these lateral reactions or lateral displacement of bottom flanges depends on the degree of lateral constraint.

The 3D FEM models in this dissertation uses lateral constraints to restrain the lateral movement of bottom flanges. A lateral restraint results in development of a lateral reaction $\left(\mathrm{R}_{\mathrm{Z}}\right)$ that is evaluated for girders of Bridge $\mathrm{A}$ for different detailing methods at different loading stages and are shown in Figure 2.10. Lateral reactions for the Final Fit detailing method at the SDL stage are equal and opposite to the lateral reactions for the Erected Fit detailing method at the TDL stage. 

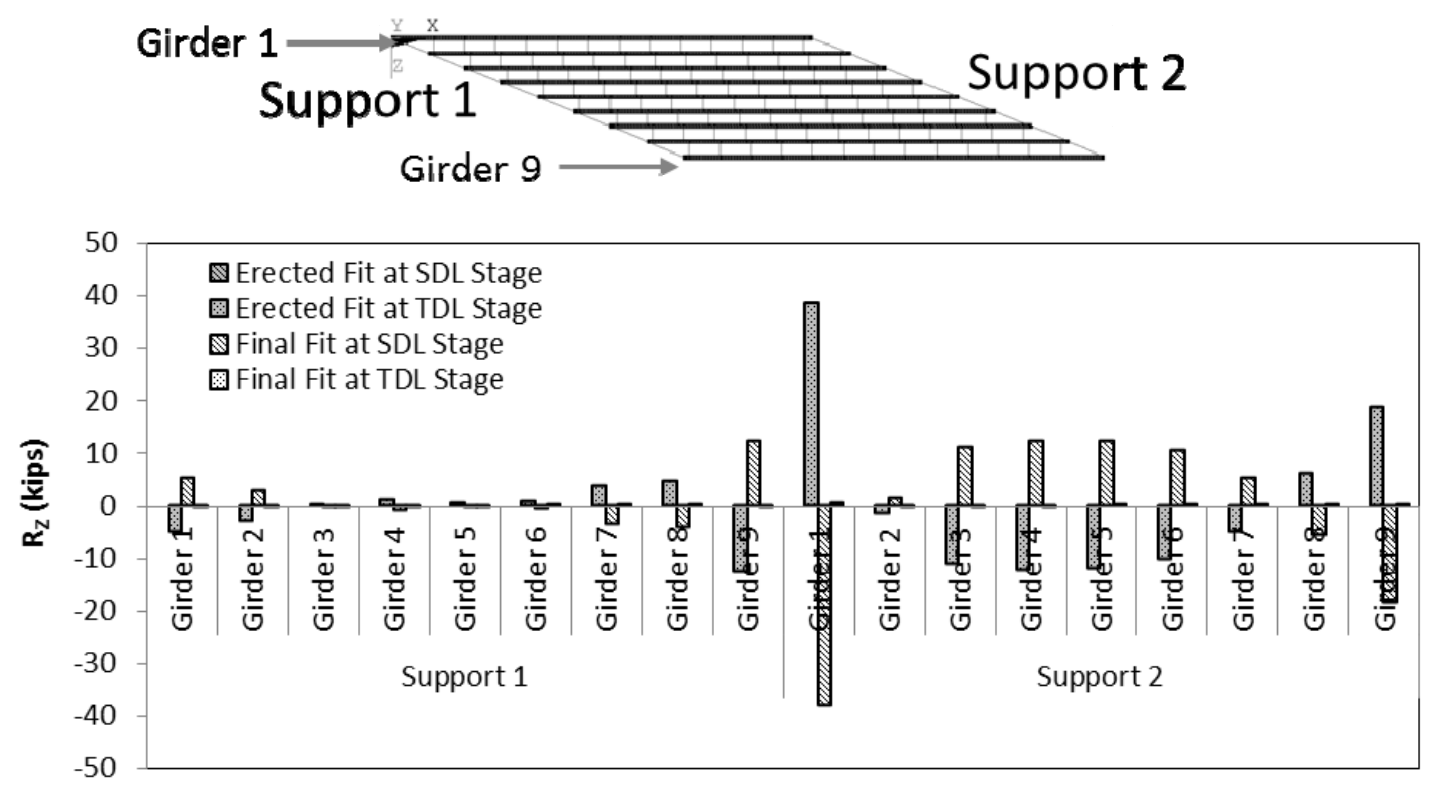

Figure 2.10: Lateral reactions $\left(\mathrm{R}_{\mathrm{Z}}\right)$ of Bridge $A$ for different detailing methods at different loading stages

\subsubsection{The Flange Lateral Bending Stress}

The flange lateral bending stress $\left(f_{l}\right)$ needs to be checked both for the Erected Fit and the Final Fit detailing method in order to meet AASHTO [3] requirements. As stated in earlier, 3D FEM analyses with flanges modeled with shell elements are used to obtain the results presented in this chapter. $f_{l}$ is calculated for the top flange of bridge girders. In order to obtain $f_{l}$, the mean value of the longitudinal stress at the two edges of the top flange is subtracted for the longitudinal stress at one of the edge of the top flange.

$f_{l}$ in top flange of Girder 1 of Bridge A is obtained from the 3D FEM analysis is for different detailing methods at different loading stages of construction and is shown in 
Figure 2.11. The following observations can be made by inspecting the data presented in Figure 2.11:

- For the Final Fit detailing method, $f_{l}$ is close to zero at the TDL stage. This behavior is because of the fact that for the Final Fit detailing method, the crossframes are fabricated to fit between their connections to girders at the TDL stage. Therefore, these cross-frames do not apply any lateral load on girders at the TDL stage. The lateral loads applied by the cross-frames to the flanges due to lack-offit at the SDL stage are the main reason for $f_{l}$ in skewed bridges.

- At the SDL stage, $f_{l}$ has a significant magnitude for the Final Fit detailing method.

- For the Erected Fit detailing method, the cross-frames are fabricated to fit between their connections to girders at the SDL stage and do not fit between their connections to girders at the TDL stage. Therefore, the corresponding crossframes forces act on the bridge girders at the TDL stage and result in $f_{l}$ at the TDL stage.

- At the SDL stage, $f_{l}$ is close to zero for the Erected Fit detailing method.

- It can be noted that $f_{l}$ for the Erected Fit detailing method at the TDL stage is almost equal and opposite to $f_{l}$ for the Final Fit detailing method at the SDL stage. Similar observations were observed for other bridges analyzed as part of this study.

The flange major axis bending stress, $f_{b}$ is higher at the TDL stage compared to the $f_{b}$ at the SDL stage for both detailing methods. $f_{l}$ for the Final Fit detailing method can be less critical compared to $f_{l}$ for the Erected Fit detailing method as it appears when the $f_{b}$ is relatively low. However, if wind loads are significant then $f_{l}$ for the Final Fit detailing method can be more critical compared to $f_{l}$ for the Erected Fit detailing method. 


\section{Girder 1}
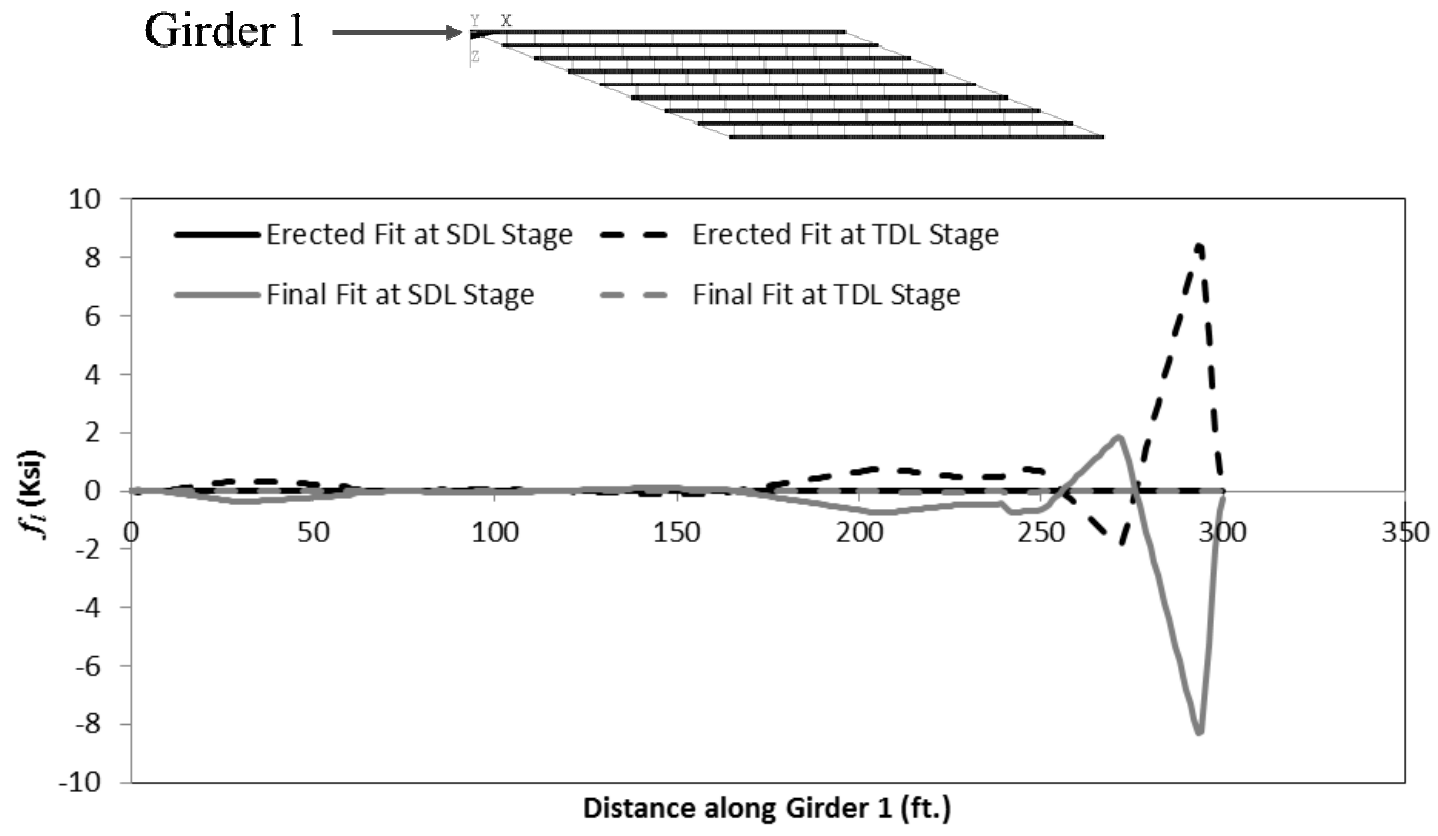

Figure 2.11: Flange lateral bending stress in top flange of Girder 1 of Bridge $A$ for different detailing methods at different loading stages

\subsubsection{Cross-frame Forces}

In general, the cross-frame forces for the Erected Fit at the TDL stage are equal and opposite to the cross-frame forces for the Final Fit at the SDL stage. It should be noted that skewed steel bridges have been constructed successfully for many years, and to the author's knowledge there have been no reported field problems with the cross-frame forces.

Forces in top chord members of cross-frames in Bay 7 of Bridge A are obtained from the 3D FEM analysis for different detailing methods at different loading stages of the construction and are shown in Figure 2.12. The following observations can be noted by inspecting the data presented in Figure 2.12: 
- For the Final Fit detailing method, the cross-frame forces are very small at the TDL stage. This behavior is because of the fact that for the Final Fit detailing method, the cross-frames are fabricated to fit between the connections to girders at the TDL stage. Therefore, these cross-frames do not develop forces.

- For the Erected Fit detailing method the cross-frames are fabricated to fit between their connections to girders at the SDL stage and do not fit between their connections to girders at the TDL stage. Therefore, these cross-frames develop significant forces at the TDL stage.

- At the SDL stage, the cross-frame forces are zero for the Erected Fit detailing method and are significant for the Final Fit detailing method.

Similar observations were observed for other bridges analyzed as part of this study.

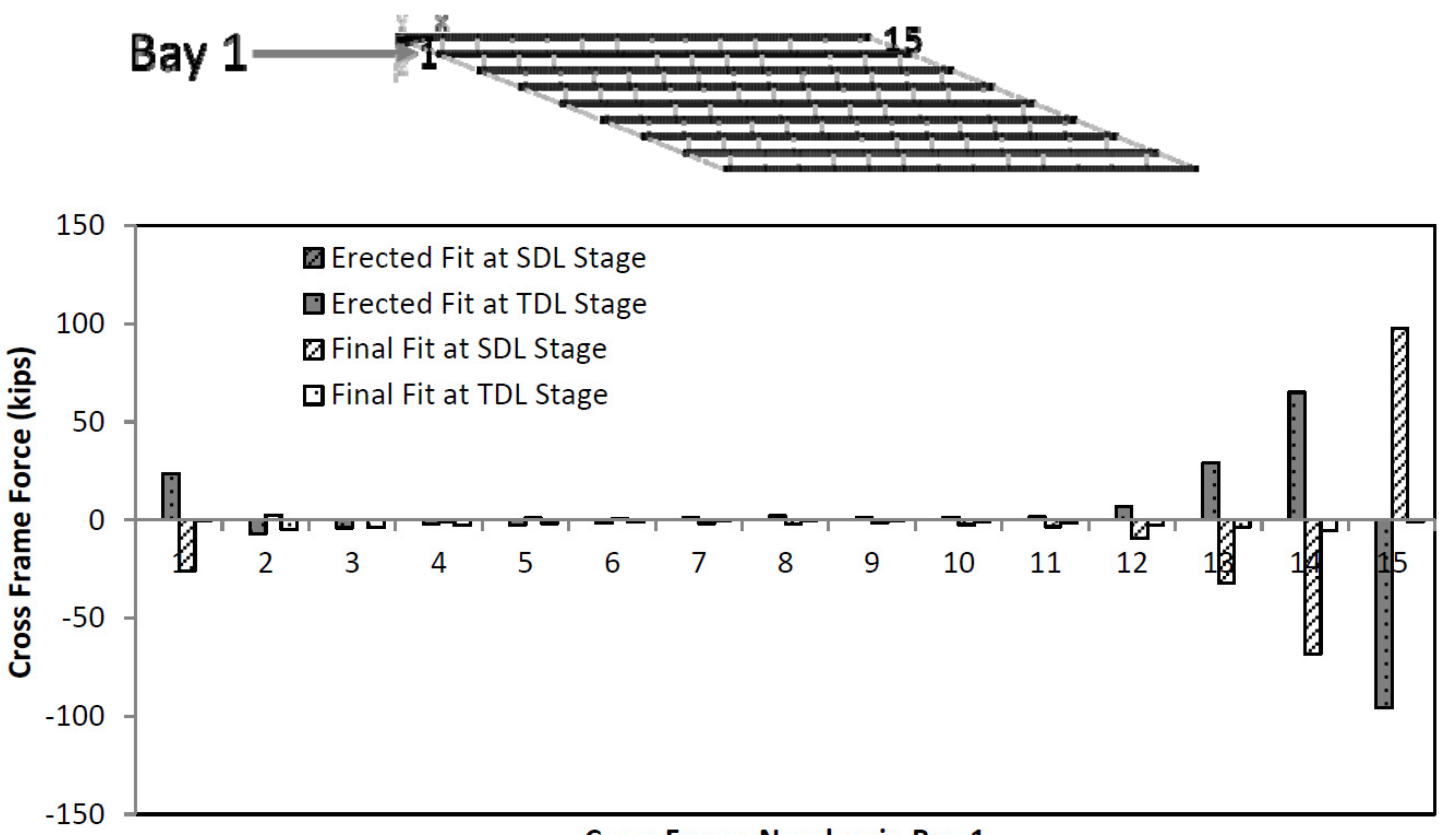

Cross Frame Number in Bay 1

Figure 2.12: Cross-frame forces in top chord of cross-frames in Bay 7 of Bridge A for different detailing methods at different loading stages 


\subsection{Discussion of the Results}

The comparison of the Erected Fit and the Final Fit presented in section 2.5.1 to section 2.5.5 shows that lack-of-fit effects for the Final Fit detailing method at the SDL stage are equal and opposite to lack-of-fit effects for the Erected Fit detailing method at the TDL stage. This observation can be explained by Figure 2.13 assuming the in-plane stiffness of the cross-frames to be very large compared to the torsional stiffness of the girders.

Before explaining the equal and opposite lack-of-fit shown in Figure 2.13, following facts are worth noting:

1. The cross-frame members' length for the Final Fit detailing method is different from the cross-frame members' length for the Erected Fit detailing method as shown in Figure 2.13. Regardless of these small differences in the length of crossframe members in different detailing methods, the assumption that in-plane stiffness of cross-frames is very large compared to torsional stiffness of the girders stands correct.

2. In a linear elastic static analysis of the steel bridge frame under the dead loads, girders with or without attaching cross-frames do not deflect by the elastic lateral torsional buckling. Further, including the initial imperfection and the material nonlinearity in the static analysis cannot capture elastic buckling modes of the bridge frame.

Keeping the above two facts in mind, Figure 2.13 shows the Erected Fit and the Final Fit detailing method at different loading stages. For the Erected Fit detailing method the cross-frame fit between the girders at the SDL stage as shown in Figure 2.13 (a). However, if the concrete dead load is applied without connecting the girders by cross-frames; the girders get deflected following a line or isolated girder vertical 
deflections and assume a configuration shown in Figure 2.13 (b). Notice the distance between cross-frames and their connections $(\Delta)$ shown in Figure 2.13 (b) is from application of the concrete dead load on line or isolated girders. For the Final detailing method cross-frames are detailed to fit between their connections to girders at the TDL stage as shown in Figure 2.13 (d). However, if the concrete dead load is removed without connecting the girders by cross-frames; the girders get deflected following line or isolated girder vertical deflections and assume a configuration shown in Figure 2.13 (c). Notice the distance between cross-frames and their connections to girders $(\Delta)$ shown in Figure 2.13 (c) is from the removal of the concrete dead load on line or isolated girders.

Since, $\Delta$ for both Erected Fit and Final Fit is from the line or isolated girder analysis, it can be stated that $\Delta$ at the TDL stage for the Erected Fit detailing method is equal and opposite to $\Delta$ at the SDL stage for the Final Fit detailing method.

Lack-of-fit effects appear for the Erected Fit detailing at the TDL stage and the Final Fit detailing method at the SDL stage only when cross-frames are connected to the girders. Given the equal and opposite distances between cross-frames and their connections to the girders for these detailing methods at these loading stages, the lack-offit effects are also equal and opposite for these detailing methods at these loading stages.

It is important to note that the lack-of-fit effects also include a component of the vertical deflection. For example if the concrete dead load is applied on girders after attaching cross-frames, detailed with Erected Fit detailing method, the vertical deflection of a girder includes a component of the vertical deflection due to the lack-of-fit. Therefore, these vertical deflections should not be used to simulate lack-of-fit effects for the Final Fit detailing method. Using these vertical deflections to simulate lack-of-fit 
effects in the Final Fit detailing method is equivalent to considering the lack-of-fit effect twice in the Final Fit detailing method and results in erroneous responses.

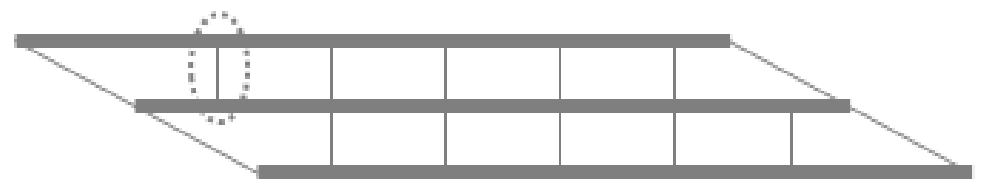

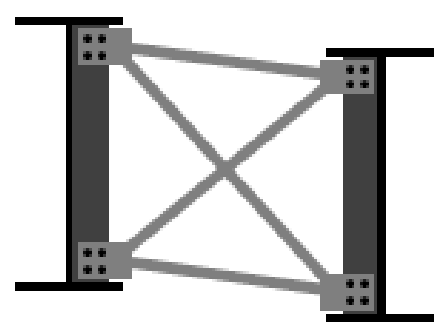

(a) Erected Fit at SDL stage

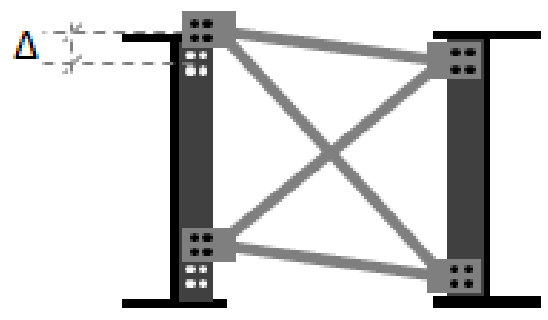

(b) Erected Fit at TDL stage

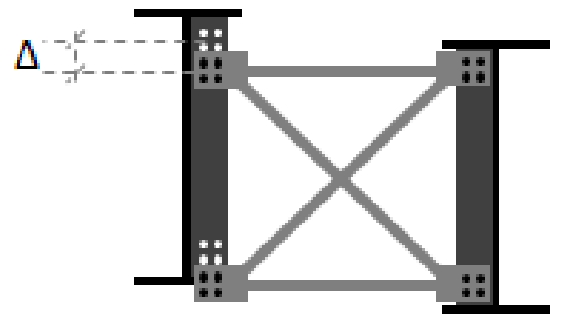

(c) Final Fit at SDL stage

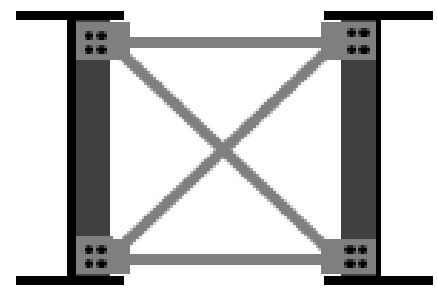

(d) Final Fit at TDL stage

\section{Figure 2.13: Equal and opposite lack-of-fit}

It is important to distinguish the mechanism explained above from the mechanism explained in NCHRP 725 [9]. In order to be consistent with NCHRP 725, terminologies used in NCHRP 725 [9] for different detailing methods are used in the following discussion. Moreover, it is convenient to compare and explain the mechanism in terms of the no load fit and the steel dead load fit (Erected Fit) from the practical stand point. 
Cross-frame forces development mechanism explained in NCHRP 725 [9] is shown in Figure 2.14. The cross-frame forces generally develop in all the cross-frame members; however, in Figure 2.14 cross-frame force is shown in only one member of the cross-frame in order to keep the figure simple. For the no load fit (NLF) there is no force in the cross-frame at the no load (NL) stage as shown in Figure 2.14 (a). The cross-frame forces appear due to application of the steel dead load (SDL) for the NLF at the SDL stage as shown in Figure 2.14 (b).

For the steel dead load fit (SDLF) the cross-frames do not fit between their connections to the girders at the NL stage. This situation is described as initial lack-of-fit in NCHRP 725[9] as shown in Figure 2.14 (c). Locked-in forces developed in the crossframes due to initial lack-of-fit after the cross-frames are forced to make connections with the girders as shown in Figure 2.14 (d). Once the SDL is applied, the cross-frame forces developed due to the SDL try to balance the locked-in forces for the SDLF at the SDL stage as shown in Figure 2.14 (e).

Although, the mechanism of developing cross-frame forces explained in NCHRP 725 [9] is consistent, however, it fails to answer: 1) why cross-frame forces for the NLF at the SDL stage are equal and opposite to the cross-frame forces for the SDLF at the NL stage?, 2) why girders need to be cambered using the line girder analysis (LGA) or the isolated girder analysis (IGA) for the SDL when the SDLF is used for detailing the crossframes. Answers to these questions and difference of the mechanism presented in this dissertation to the mechanism explained in NCHRP 725 [9] are made clear in the following discussion. 


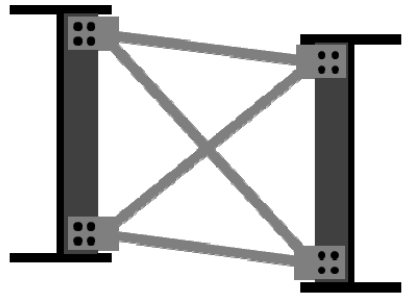

(a) NLF at NL stage

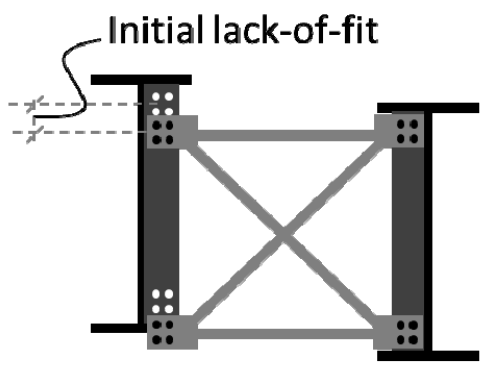

(c) SDLF at NL stage before connecting cross-frames

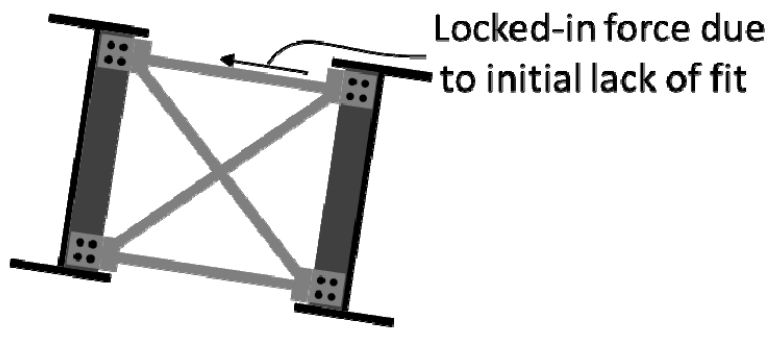

(d) SDLF at NL stage after connecting cross-frames

(b) NLF at SDL stage

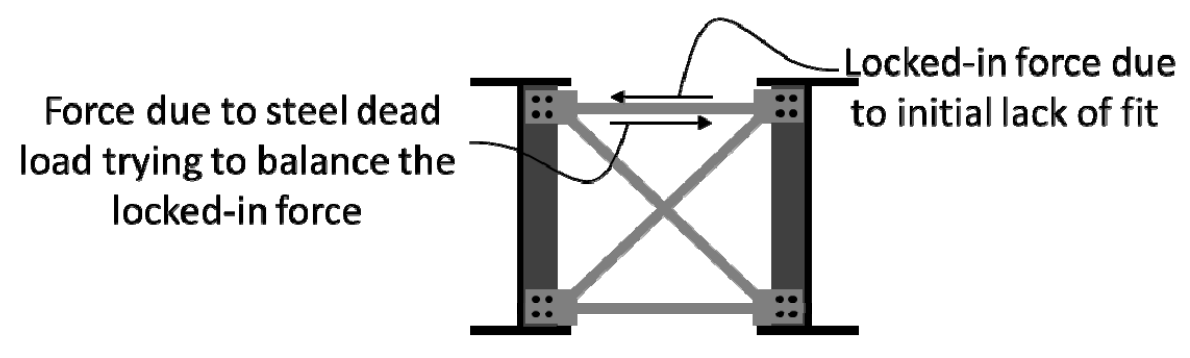

(e) SDL at SDL stage

Figure 2.14: Cross-frame forces development mechanism explained in NCHRP 725

Figure 2.15 shows the mechanism by which cross-frames forces develop due to the lack-of-fit of cross-frames between their connections to the girders regardless of the detailing method. Figure 2.15 has an additional stage for the NLF at the SDL stage that represents a hypothetical situation in which girders are placed at supports but crossframes are not connected to girders. 
The mechanism explained in this research postulates that cross-frames forces, similar to other lack-of-fit effects, develop after connecting the cross-frames to the girders at a loading stage in which there is a lack-of-fit between the cross-frames and their connections to girders. Magnitude of cross-frame forces is proportional to the lackof-fit similar to the magnitude of other lack-of-fit effects for a given bridge geometry and member sizes. The application or the removal of the dead load (the steel dead load or concrete dead load or total dead load) moves the girders to different vertical positions creating or removing the lack-of-fit. The dead load does not cause any force in the crossframes directly as mentioned in NCHRP 725 [9] instead it is the lack-of-fit of crossframes between their connection to girders that produces the cross-frame forces and other lack-of-fit effects. This fact is further explained through Figure 2.15.

For the NLF at the NL stage there is no force in the cross-frames because there is no lack-of-fit between the cross-frames and their connection points to the girders as shown in Figure 2.15 (a). This situation is very similar to the SDLF at the SDL stage and there is no force in the cross-frames because there is no lack-of-fit between the crossframes and their connections to the girders as shown in Figure 2.15 (f).

Once the SDL is applied for the NLF without connecting the cross-frames, the SDL move the girders to a different position in which cross-frames do not fit between their connections to the girders as shown in Figure 2.15 (b). This situation is very similar to the SDLF at the NL stage where removal of the steel dead load from isolated girder creates a lack-of-fit between cross-frames and their connection to the girders. Notice removal of the dead load from isolated girders would create a lack-of-fit that is equal to 
and apposite to the lack of created by application of dead load on isolated girders as shown in Figure 2.15 (b) and Figure 2.15 (d).

If cross-frames are connected to the girders for NLF at SDL there are forces in cross-frames due to the lack-of-fit as shown in Figure 2.15 (c). This situation is very similar to the SDL at the NL stage as shown in Figure 2.15 (e). Since, magnitude of cross-frame forces is proportional to the lack-of-fit for a given bridge geometry and member sizes, the cross-frame forces for the NLF at the SDL stage are equal and opposite to the cross-frame forces for the SDLF at the NL stage.

The difference between the vertical deflection of isolated girders and the girders attached together with cross-frames is the component of the vertical deflection due to lack-of-fit $\left(\mathrm{D}_{\mathrm{Y} 2}\right)$ as explained earlier. Since $\mathrm{D}_{\mathrm{Y} 2}$ is zero for the SDLF at the SDL, therefore the girders should be camber using isolated or line girder analysis for SDL when using SDLF. 


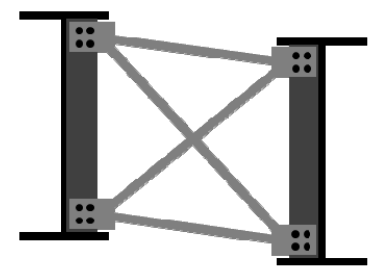

(a) NLF at NL stage

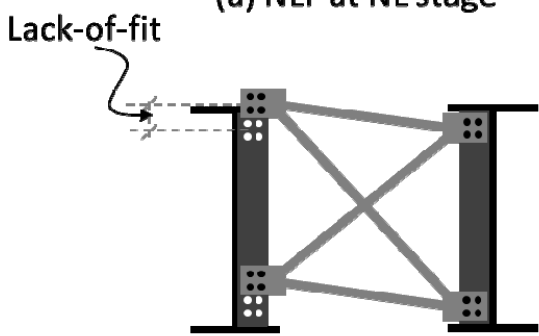

(b) NLF at SDL stage before connecting cross-frames

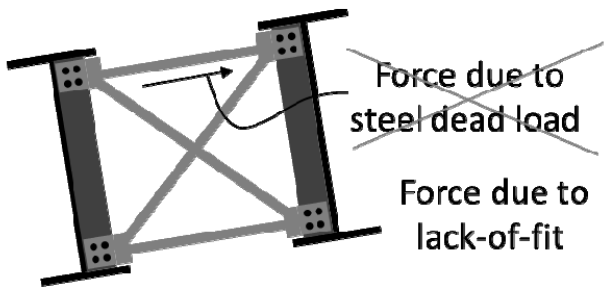

(c) NLF at SDL stage after connecting cross-frames

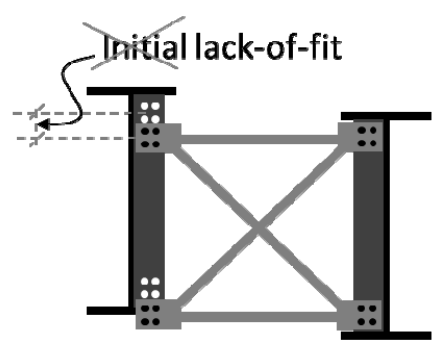

(d) SDLF at NL stage before connecting cross-frames

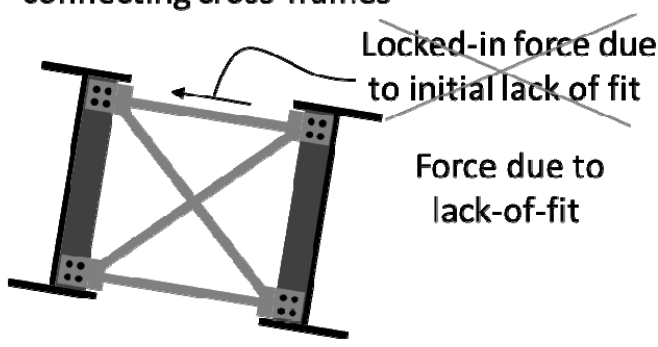

(e) SDLF at NL stage after connecting cross-frames

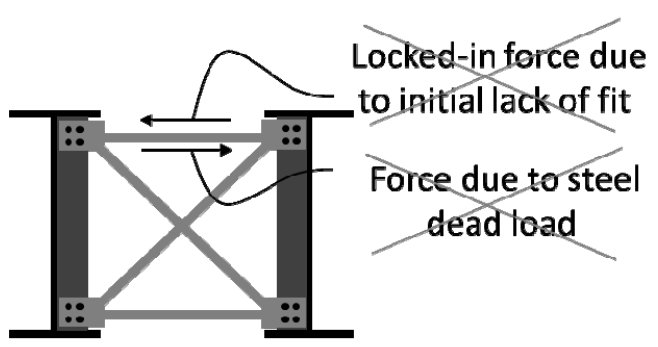

(f) SDL at SDL stage

Figure 2.15: Cross-frame forces development mechanism

\subsection{Bridges with unequal skew}

The conclusion that the lack-of-fit effects for the Final Fit detailing method at the SDL stage are equal and opposite to the lack-of-fit effects for the Erected Fit detailing method at the TDL stage is also valid for straight bridges with supports having unequal skew. The detailed comparison of detailing method at different loading stages for a straight bridge with unequal skew is shown in Appendix B. 


\subsection{Horizontally curved bridges}

Cross-frames in horizontally curved bridges can be detailed to fit between the girders at the no load (NL) stage only. The cross-frames in the curved bridges cannot be detailed to fit at the steel dead load (SDL) stage or the total dead load (TDL) stage due to following reasons:

- Isolated curved girders without cross-frames attached have static instability compared to isolated straight girders that do not have a static instability.

- For detailing the cross-frame using the SDLF or the TDLF, line or isolated girder analysis cambers are required. For a curved girder, an isolated or a line girder analysis cannot be obtained because of the static instability of the isolated curved girder.

- Isolated curved girders can be made statically stable by placing the torsional constraint at supports. However, analysis with these boundary conditions results in excessive twist of the girders in the free span after application of dead load. It is not useful to detail the cross-frames to fit between excessively twisted girders under dead load.

It is important to note that both straight and curved girders have buckling instability in absence of lateral support (cross-frames or diaphragms). However, this buckling instability is entirely a different phenomenon and cannot be considered in a simple 1D line girder static analysis.

\subsection{Summary}

This chapter describes two detailing methods (the Erected Fit and the Final Fit) used for detailing cross-frames in straight skewed I-girder Bridges. Different structural responses affected by the two detailing method at different construction stages of skewed bridges are identified. These structural responses or component of these structural 
responses are called as the lack-of-fit effects. Most important conclusion in this chapter is that the lack-of-fit effects for the Final Fit detailing method at the steel dead load stage are equal and opposite to the lack-of-fit effects for the Final Fit detailing method at the total dead load stage.

Numerical analysis results shown in this chapter are obtained from 3D FEM analyses. However, 3D FEM analyses are generally avoided in practice because of their cost and complexity as explained in Chapter 1 . The next chapter compares the lack-of-fit effects calculated from 3D FEM analyses to the lack-of-fit effect calculated from simplified analyses to recommend simplified methods of analysis for calculating these lack-of-fit effects. 


\section{METHODS OF ANALYSIS FOR DIFFERENT DETAILING METHODS}

Different methods of analysis that are used for steel bridges include traditional 2D Grid Analysis (GA), the improved 2D GA, and 3D FEM analysis [1] [2] [3][6] [7] [8]. Currently, the 2D GA can be used for the no load fit detailing method only, and a 3D FEM analysis, with initial strains in the cross-frame members to simulate the lack-of-fit, is required for dead load fit (Erected Fit and Final Fit) detailing methods.

The objective of this chapter is to introduce different methods of analysis that can be used to calculate the lack-of-fit effects for the Erected Fit detailing method at the TDL stage and the Final Fit detailing method at the SDL stage. The comparison of different methods is done to recommend a single simplified method of analysis that can be used to calculate the lack-of-fit effects with reasonable accuracy for both Erected Fit and Final Fit detailing method.

\subsection{Method of analysis for the Erected Fit Detailing Method}

Different methods of analysis that are used for calculation of different structural responses for the NLF detailing method are discussed at length in NCHRP 725 [3]. A brief summary of these methods is provided here.

\subsubsection{D Line Girder Analysis}

In 1D line girder analysis (LGA) the girders are analyzed as line elements without any cross-frame attached to them. 1D LGA mentioned in this chapter refers to the numerical analysis of a bridge girder modeled with line elements. 


\subsubsection{D Grid Analysis}

Generally, 2D grid analysis (GA) models the cross-frames and girders with the elements having three degrees of freedom at each node. However, the 2D GA used in this chapter refers to a modeling technique in which each node has six degrees of freedom (three translations and three rotations), but entire structural model of the bridge is in a single horizontal plane. This chapter uses two types of 2D GA, the traditional 2D GA, and the improved 2D GA.

The torsional stiffness of the girders is estimated by the St. Venant term using the torsional constant $(J)$ in the traditional 2D GA. In the improved 2D GA, the torsional stiffness of the girder is modeled by using an equivalent torsional constant $\left(J_{e q}\right)$ that takes into account both the St. Venant and warping terms in the calculation of the torsional stiffness. The detailed expressions for obtaining $J_{e q}$ for I-sections are given in the literature [17]. These expressions are derived based on the assumption that both ends of the unbraced length $\left(L_{b}\right)$ are either fix-fix or fix-pin.

$$
\begin{gathered}
J_{e q_{(f x-f x)}}=J\left[1-\frac{\sinh \left(p L_{b}\right)}{p L_{b}}+\frac{\left[\cosh \left(p L_{b}\right)-1\right]^{2}}{p L_{b} \sinh \left(p L_{b}\right)}\right]^{-1} \\
J_{e q_{(f x-p i n)}}=J\left[1-\frac{\sinh \left(p L_{b}\right)}{p L_{b} \cosh \left(p L_{b}\right)}\right]^{-1}
\end{gathered}
$$


Where

$$
p=\sqrt{\frac{G J}{E C_{w}}}
$$

$G$ is the modulus of rigidity and can be approximated by $G=\frac{E}{2(1+v)}, E$ is the modulus of elasticity of the material, $v$ is Poisson's ratio, and $C_{w}$ is the warping constant.

Following expression can be used for calculating $J$ and $C_{w}$ for an I-section.

$$
\begin{gathered}
J=\frac{1}{3}\left(b_{t f} t_{t f}^{3}+h_{w} t_{w}^{3}+b_{b f} t_{b f}^{3}\right) \\
C_{w}=\frac{h_{w}^{2} b_{b f}^{3} t_{b f}}{12\left(1+\left(\frac{b_{b f}}{b_{t f}}\right)^{3}\left(\frac{t_{b f}}{t_{t f}}\right)\right)}
\end{gathered}
$$

In the above expressions, $h_{w}$ is height of web, $b_{t f}$ and $b_{b f}$ is width of top flange and bottom flange respectively, and $t_{t f}$ and $t_{b f}$ is thickness of top flange and bottom flange respectively.

A cross-frame in the $2 \mathrm{D}$ GA is modeled using a beam element with a moment of inertia $\left(I_{e q}\right)$ that matches the flexural stiffness of the truss representation of the crossframe. The beam also has a cross section area $\left(A_{e q}\right)$ that matches the axial stiffness of the cross-frame system. The traditional 2D GA uses the Euler Bernoulli beam stiffness matrix whereas the improved 2D GA employed here uses an equivalent beam stiffness that matches the stiffness of a truss idealization of the cross-frames exactly within their plane. Detailed derivations and expressions for these stiffness matrices are provided in [7] and [9]. In order to clarify the stiffness matrix for girder and cross frames used in this 
dissertation, the expressions for stiffness matrices of girders and cross-frames are provided in Appendix C.

It should be noted that in the Erected Fit detailing method the lack-of-fit effects such as girder layovers, the component of the vertical deflection due to lack-of-fit, crossframe forces, the component of vertical reactions due to lack-of-fit, lateral reactions, and flange lateral bending stress appear after placement of the wet concrete (the TDL stage). Therefore, in order to carry out an Erected Fit analysis using the 2D grid method, a complete model of the structure is constructed with cross-frames attached to the girders followed by activating the concrete dead load (CDL) only. Structural responses obtained from the LGA for the SDL can be added to the structural responses obtained from the 2D GA for the CDL to obtain the structural responses at the TDL stage for Erected Fit detailing method.

\subsubsection{D FEM analysis}

As mentioned earlier in section 2.4, 3D FEM analysis can be used with different levels of modeling details. 3D FEM analyses employed in this study use flanges modeled with shell elements and bearing pads to model the boundary conditions. 3D FEM analysis for the Erected Fit detailing method can be accomplished by following the same steps that are described above for the 2D GA.

\subsection{Comparison of Different Methods of Analysis for the Erected Fit}

Different methods of analysis discussed in the above sections are used to evaluate the lack-of-fit effects for the Erected Fit detailing method at the SDL stage. These lackof-fit effects include girder layovers, the component of the vertical deflection due to lack- 
of-fit, the component of vertical reaction due to lack-of-fit, the flange lateral bending stress, and cross-frame forces. In the following sections each lack-of-fit effect is obtained from different methods of analysis and compared to recommend a method of analysis for calculating the lack-of-fit effects.

Three different bridges (Bridge A, Bridge B and Bridge C) were analyzed to support the discussion in this chapter. Limited results for two bridges (Bridge A and Bridge B) shown within the chapter. Detailed results are shown in Appendix C. Bridge A uses staggered cross-frames and Bridge B uses contiguous cross-frames. Details of these bridges are provided in Appendix A.

\subsubsection{Girder Layovers}

Girder layovers can be obtained from 3D FEM analysis by subtracting lateral displacement of the center of the top flange from the lateral displacement of the center of the bottom flange. For calculating girder layovers from 2D GA twist of the girder is multiplied by the height of the girder. Girder layovers, obtained from different methods of analysis, are compared for Girder 1 of Bridge A in Figure 3.1 and for Girder 1 of Bridge B in Figure 3.2 for the Erected Fit detailing method at the TDL stage. For Bridge A, the traditional 2D grid analysis does not give a good estimate of girder layovers. The difference between girder layovers, obtained from different methods of analysis, is not significant for Bridge B.

(Note: Figures 3.1 through 3.10 use 2D GA Trd to connote traditional 2D GA, and 2D GA Imp to connote improved 2D GA.) 


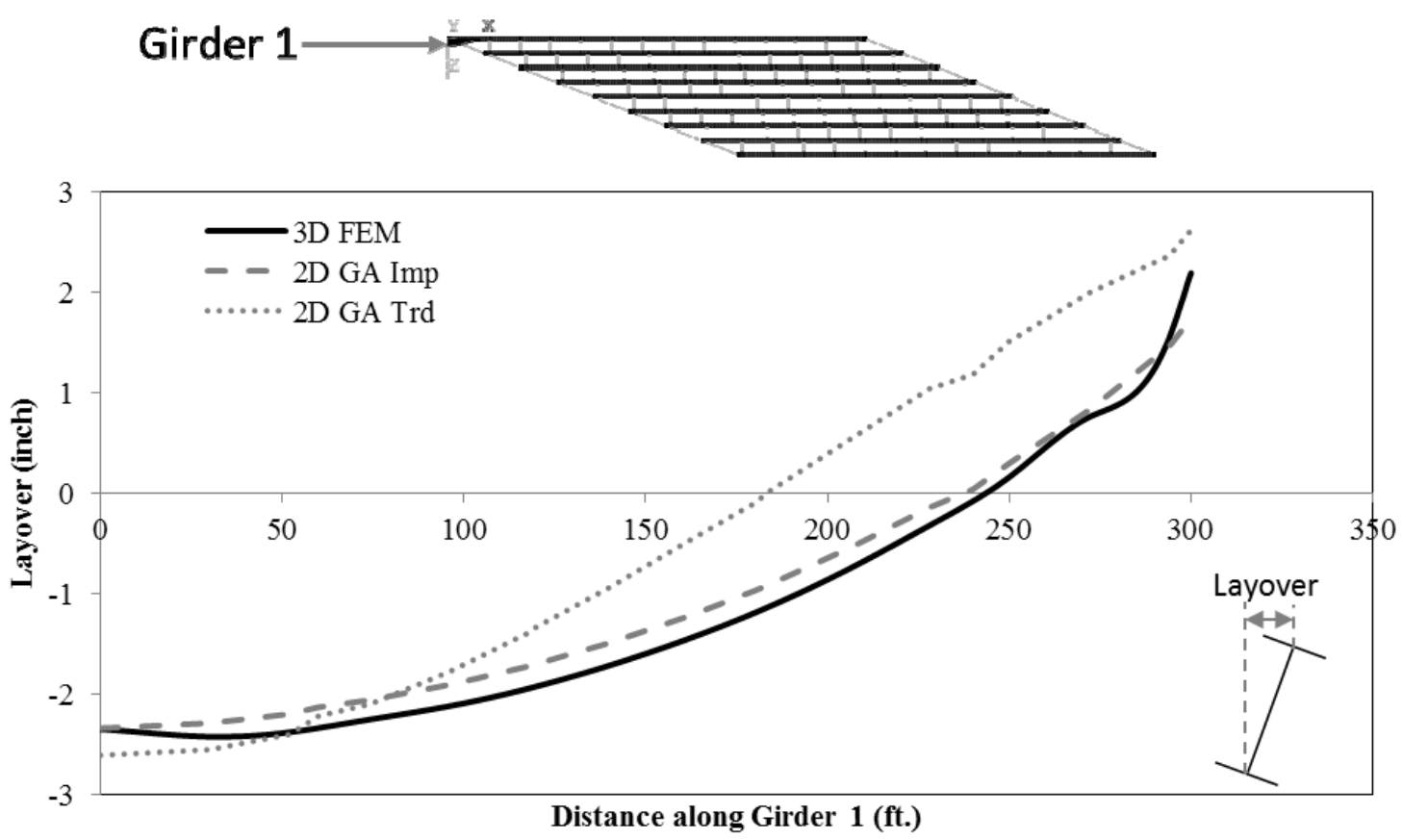

Figure 3.1: Comparison of girder layovers calculated by different analysis method for Girder 1 of Bridge A

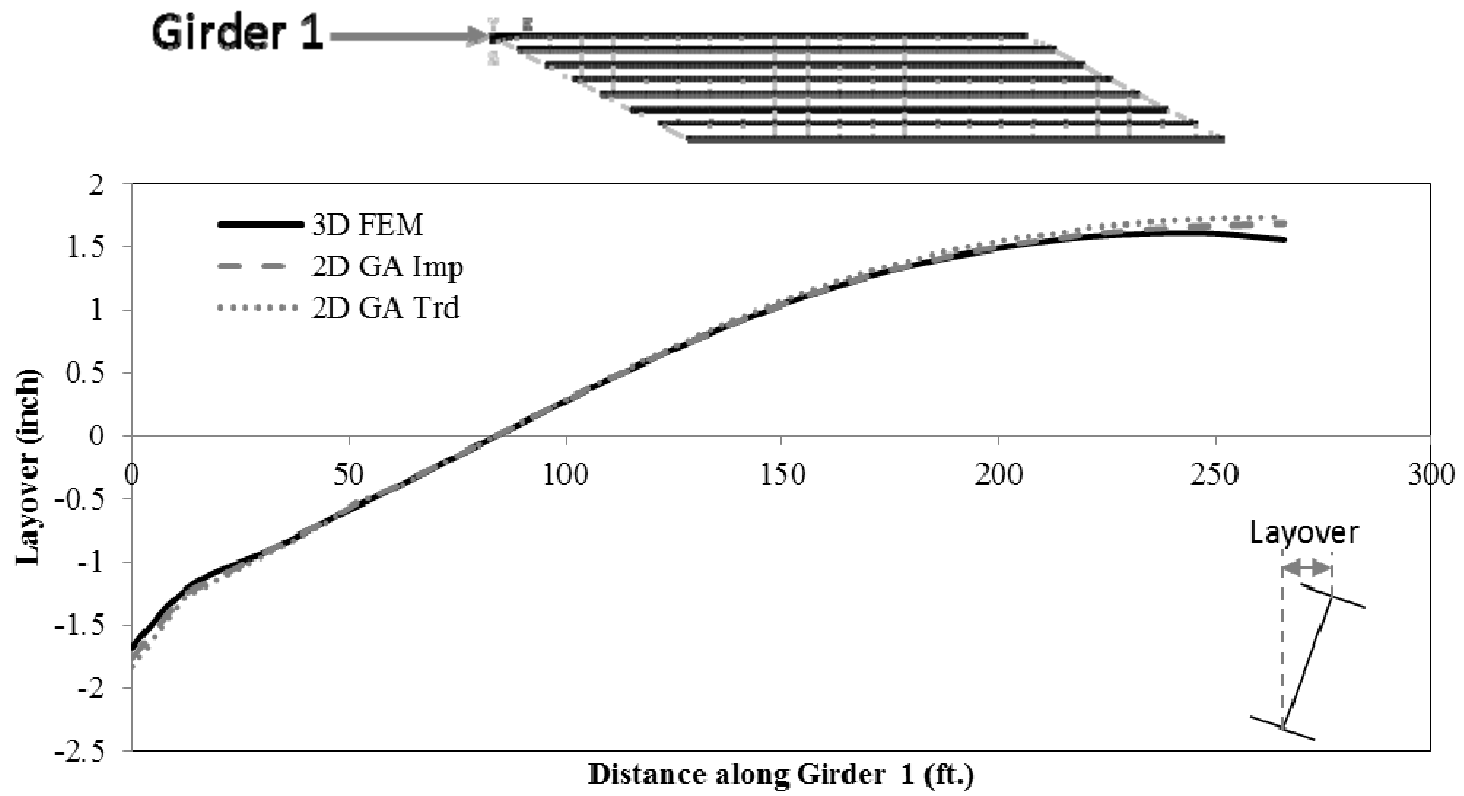

Figure 3.2: Comparison of girder layovers calculated by different analysis method for Girder 1 of Bridge B 
Traditional 2D grid analysis method gives poor estimates of girder layovers for Bridge A and good estimates of girder layovers for Bridge B. This is also true for other structural responses such as vertical reactions and cross-frame forces except for flange lateral bending stress $\left(f_{l}\right)$. This is because the cross-frames are staggered in the Bridge A compared to arrangement of cross-frames along contiguous lines in Bridge B.

When cross-frames are staggered, a cross-frame connects only two girders and forces from one cross-frame to the other cross-frame in the adjacent bay have to be transferred through the girder. On the other hand, when cross-frames are arranged in contiguous lines, cross-frame forces can be directly transferred from one cross-frame to the other cross-frame in the adjacent bay. Therefore, in staggered framing, lack-of-fit effects are dependent on the torsional stiffness of girders. Since the traditional 2D GA does not model the torsional stiffness of girders accurately, lack-of-fit affects are not estimated correctly by the traditional 2D GA.

In contiguous framing, cross-frames are arranged along contiguous lines and can directly transfer the forces along a cross-frame line without relying on torsional stiffness of girders. Therefore, lack-of-fit affects (except for the flange lateral bending stress, $f_{l}$ ) are not affected by the torsional stiffness of girders. The detailed discussion on $f_{l}$ is provided in section 3.2.4.

It is recommended that girder layovers should be calculated using the improved 2D GA rather than the traditional 2D GA, since the improved 2D GA gives better estimates of all responses both for contiguous framing and staggered framing. 


\subsubsection{Component of the Vertical Deflections Due to the Lack-of-fit}

The component of the vertical deflection due to the lack-of-fit $\left(\mathrm{D}_{\mathrm{Y} 2}\right)$ for the Erected Fit detailing method appears at the TDL stage. Generally, $\mathrm{D}_{\mathrm{Y} 2}$ is highest in interior girders and lowest in fascia girders. The component of the vertical deflection from the lack-of-fit can be obtained using Eq. (2.1) for 3D FEM analyses and 2D GAs. The $\mathrm{D}_{\mathrm{Y} 2}$ is obtained from different methods of analysis, and is compared for Girder 5 of Bridge A in Figure 3.3 and for Girder 4 of Bridge B in Figure 3.4 for the Erected Fit detailing method at the TDL stage.

For Bridge $\mathrm{A}$, traditional 2D grid analysis does not give a good estimate of $\mathrm{D}_{\mathrm{Y} 2}$. The difference between $\mathrm{D}_{\mathrm{Y} 2}$ obtained from different methods of analysis is not significant for Bridge B. Improved 2D grid analysis tends to give higher estimates of $\mathrm{D}_{\mathrm{Y} 2}$ for both bridges compared to the one obtained from 3D FEM analysis.
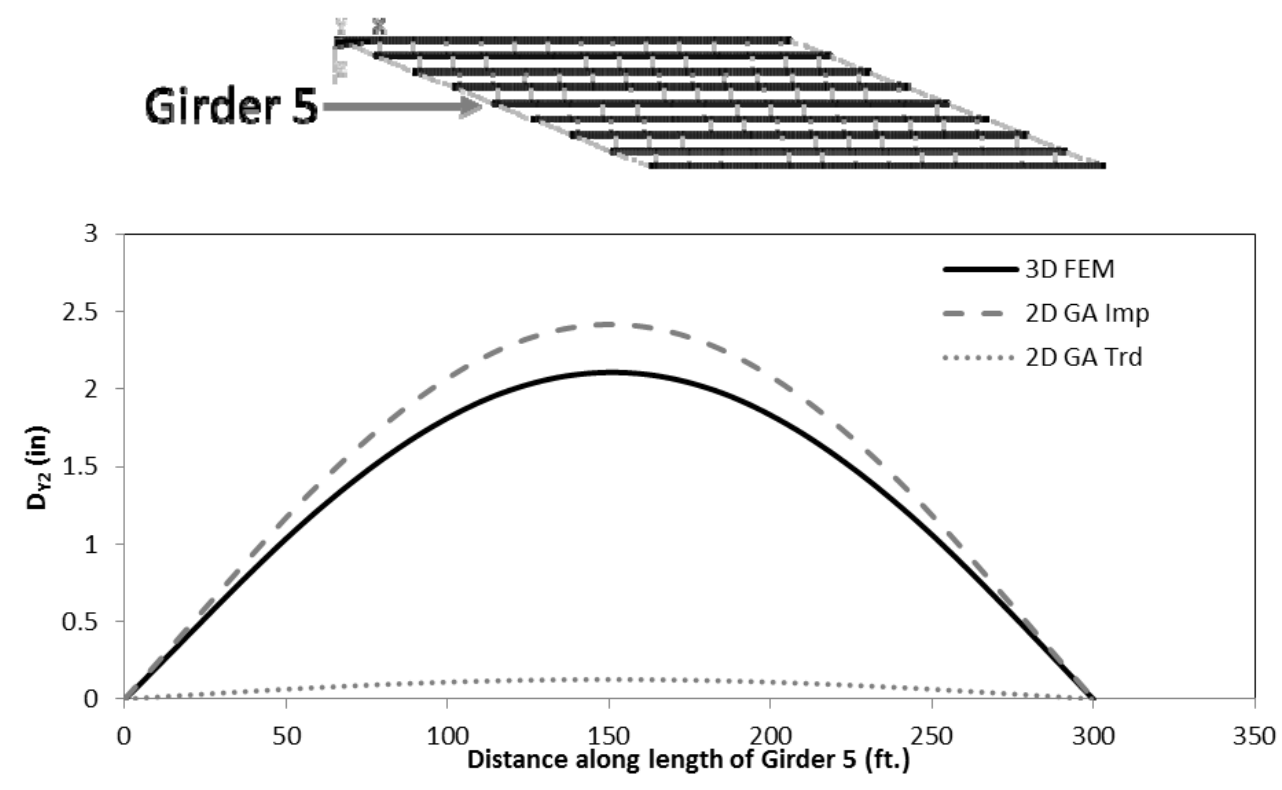

Figure 3.3: Comparison of the $D_{Y 2}$ calculated by different analysis methods for Bridge A 

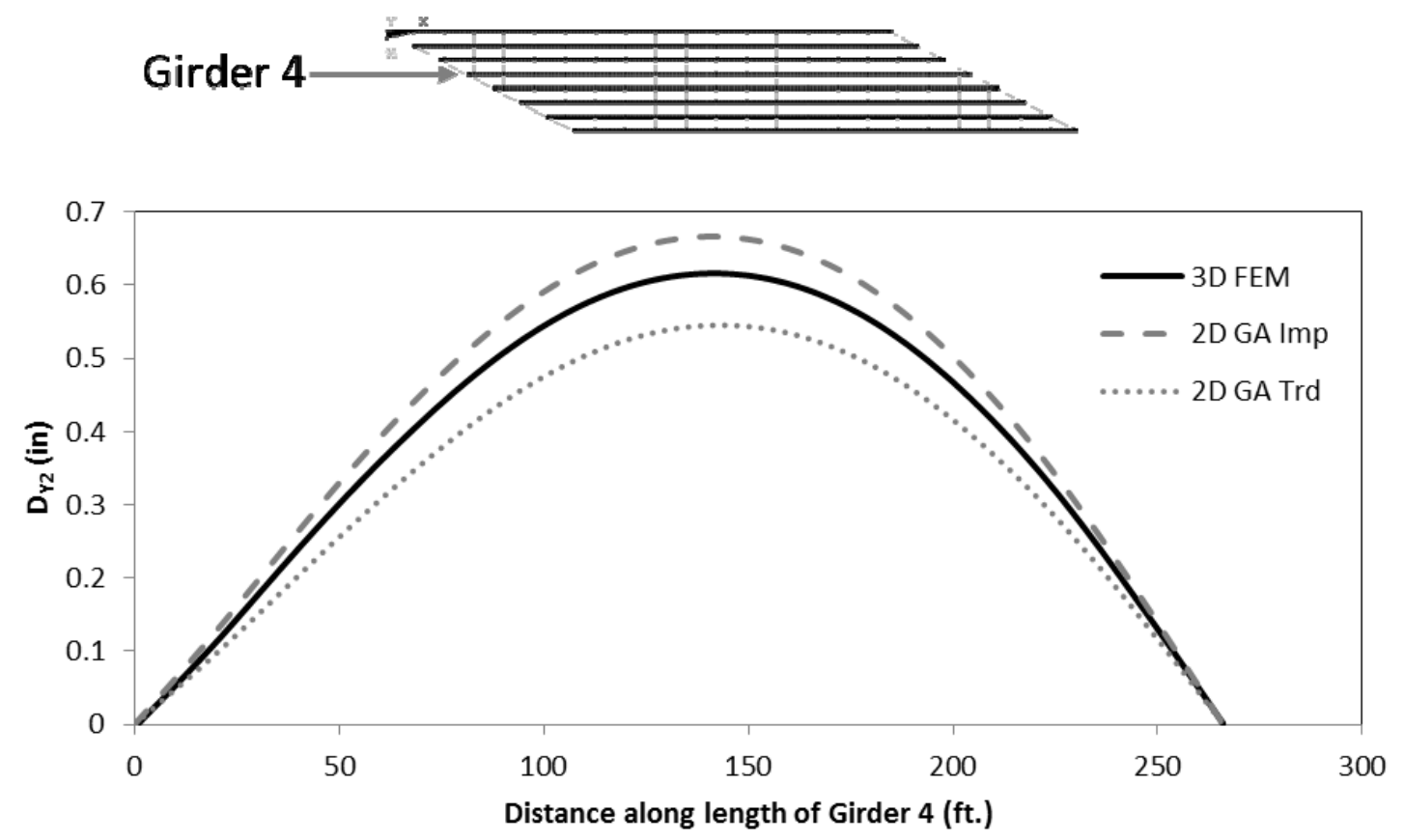

Figure 3.4: Comparison of the $D_{\mathrm{Y} 2}$ calculated by different analysis methods for Bridge $B$

\subsubsection{Component of Vertical Reactions Due to Lack-of-fit}

The component of vertical reactions due to the lack-of-fit $\left(\mathrm{R}_{\mathrm{Y} 2}\right)$ for the Erected Fit detailing method appear at the TDL loading stage. $\mathrm{R}_{\mathrm{Y} 2}$ is highest at the obtuse corners of the skewed bridge. $\mathrm{R}_{\mathrm{Y} 2}$ can be obtained using Eq. (2.2) for both 3D FEM analyses and 2D GAs. $\mathrm{R}_{\mathrm{Y} 2}$ obtained from different methods of analysis is compared for Bridge A in Figure 3.5and for Bridge B in Figure 3.6 for the Erected Fit detailing method at the TDL stage. As expected the traditional 2D grid analysis gives very low estimates of the $\mathrm{R}_{\mathrm{Y} 2}$ for Bridge A and reasonable estimates of $\mathrm{R}_{\mathrm{Y} 2}$ for Bridge $\mathrm{B}$. The improved $2 \mathrm{D}$ grid analysis gives the highest estimates of $\mathrm{R}_{\mathrm{Y} 2}$ for both bridges. It can be concluded that the improved 2-D grid analysis is sufficient to calculate $\mathrm{R}_{\mathrm{Y} 2}$. 


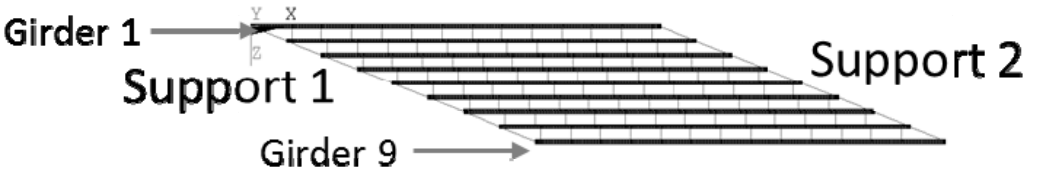

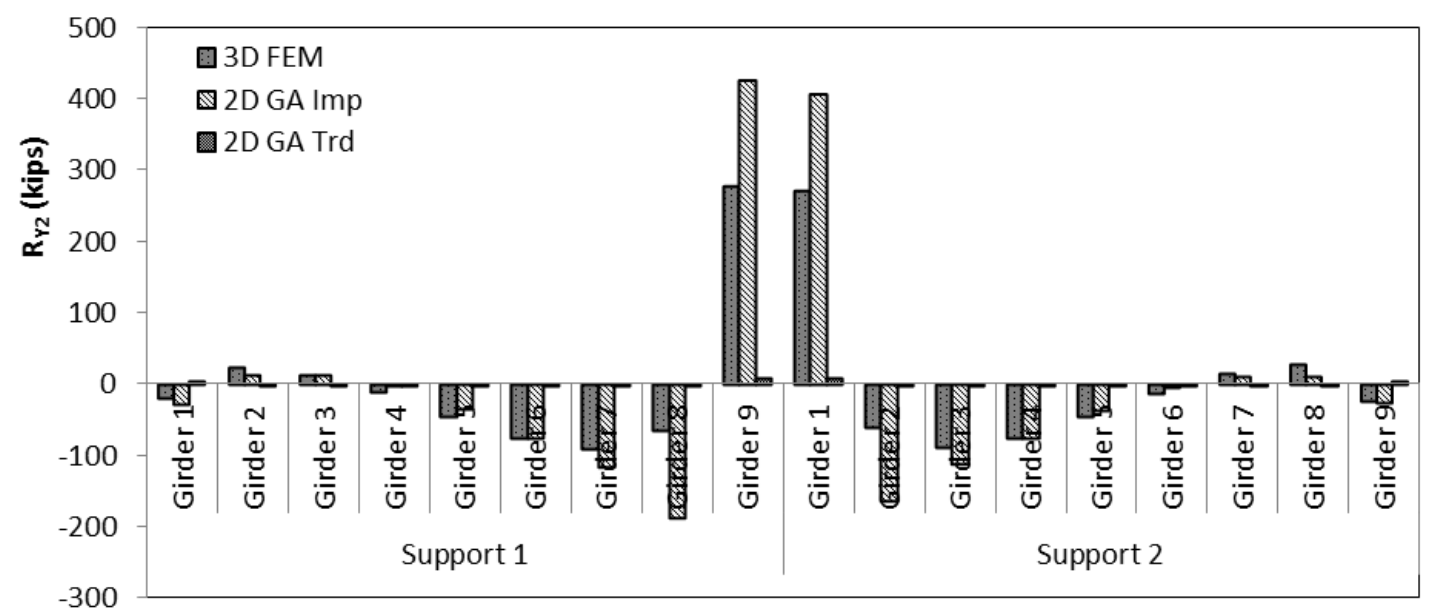

Figure 3.5: Comparison of the $R_{Y 2}$ calculated by different analysis methods for Bridge A
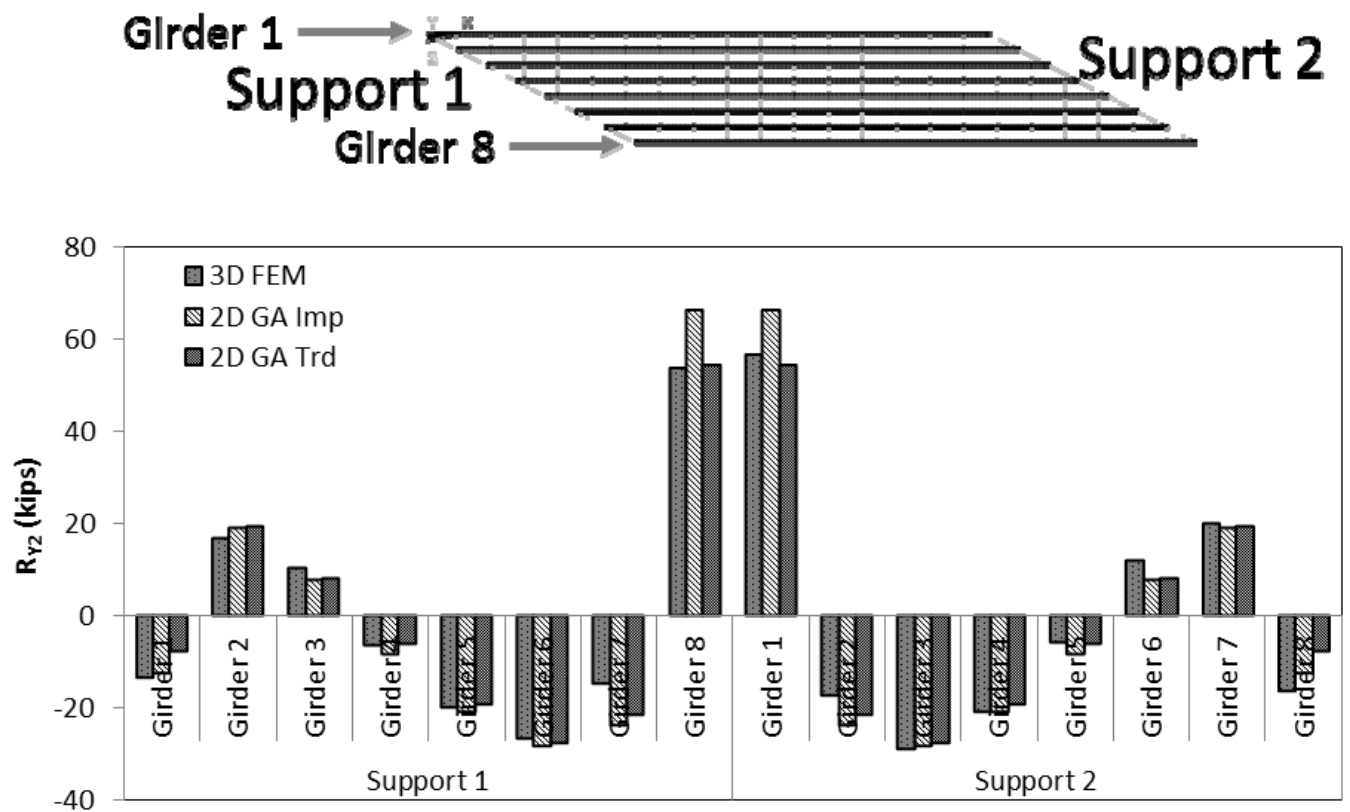

Figure 3.6: Comparison of $R_{Y_{2}}$ calculated by different analysis method for Bridge $B-$ Erected Fit at the TDL stage 


\subsubsection{Flange Lateral Bending Stress $\left(f_{i}\right)$}

The procedure to calculate the flange lateral stress from the 2D grid analysis for the Erected Fit detailing method has been specified in literature [9] and [7]. A brief summary of the procedure is provided here. Displacements corresponding to the concrete dead load from the 2D GA are used to calculate forces in cross-frame members. These forces are then resolved into vertical and lateral components at the connection point of the cross-frame and the girder. The flange is assumed simply supported or fixed ended between connections adjacent to the connection at which lateral force is obtained. Using the lateral bending moment at the location of lateral load of this idealized beam model, the lateral stress is calculated using flexural formula. In order to obtain $f_{l}$ from 3D FEM analysis, the mean value of the longitudinal stress at the two edges of the top flange is subtracted for the longitudinal stress at one of the edges of the top flange.

Flange lateral bending stresses obtained from different methods of analysis are compared for Girder 8 of Bridge A in Figure 3.7 and for Girder 4 of Bridge B in Figure 3.8 for the Erected Fit detailing method at the TDL stage. It can be noticed in both Figure 3.7and Figure 3.8 that $f_{l}$ is almost zero for the traditional 2D grid analysis that does not include warping term in modeling the torsional stiffness of girders. More appropriate values of $f_{l}$ are obtained by modeling the torsional stiffness of the girder correctly, i.e., taking into account the warping torsional stiffness. This warping torsional stiffness is incorporated into the improved 2-D grid analysis. Increase in the torsional stiffness of the girder by incorporating the warping stiffness makes the girder stiffer. The stiffness attracts more force and therefore flange lateral bending stresses increase. The effect is 
more pronounced in $f_{l}$ than in the vertical deflections since small movement can have large effect in stress.

In the 2D grid analysis two assumptions can be made for the segment of girder between three consecutive cross-frames for the calculation of the lateral moment as explained in NCHRP 725 [9]. Assuming a simply supported (s-s) boundary condition for the segment gives more value of the lateral moment and thereby conservatively estimates $f_{l}$, whereas assuming a fix-fix boundary condition for the segment gives un-conservative estimates of $f_{l}$. The boundary condition is somewhere between fix-fix and s-s in reality. However, such boundary condition is difficult to model. Results of this study indicate that the average of $f_{l}$ values obtained based on the two assumption constitutes an acceptable approach, which is in agreement with the recommendations of NCHRP 725 [9].

It can be concluded that the improved 2-D grid analysis with an average value of $f_{l}$ constitute an acceptable approach to approximate $f_{l}$. 

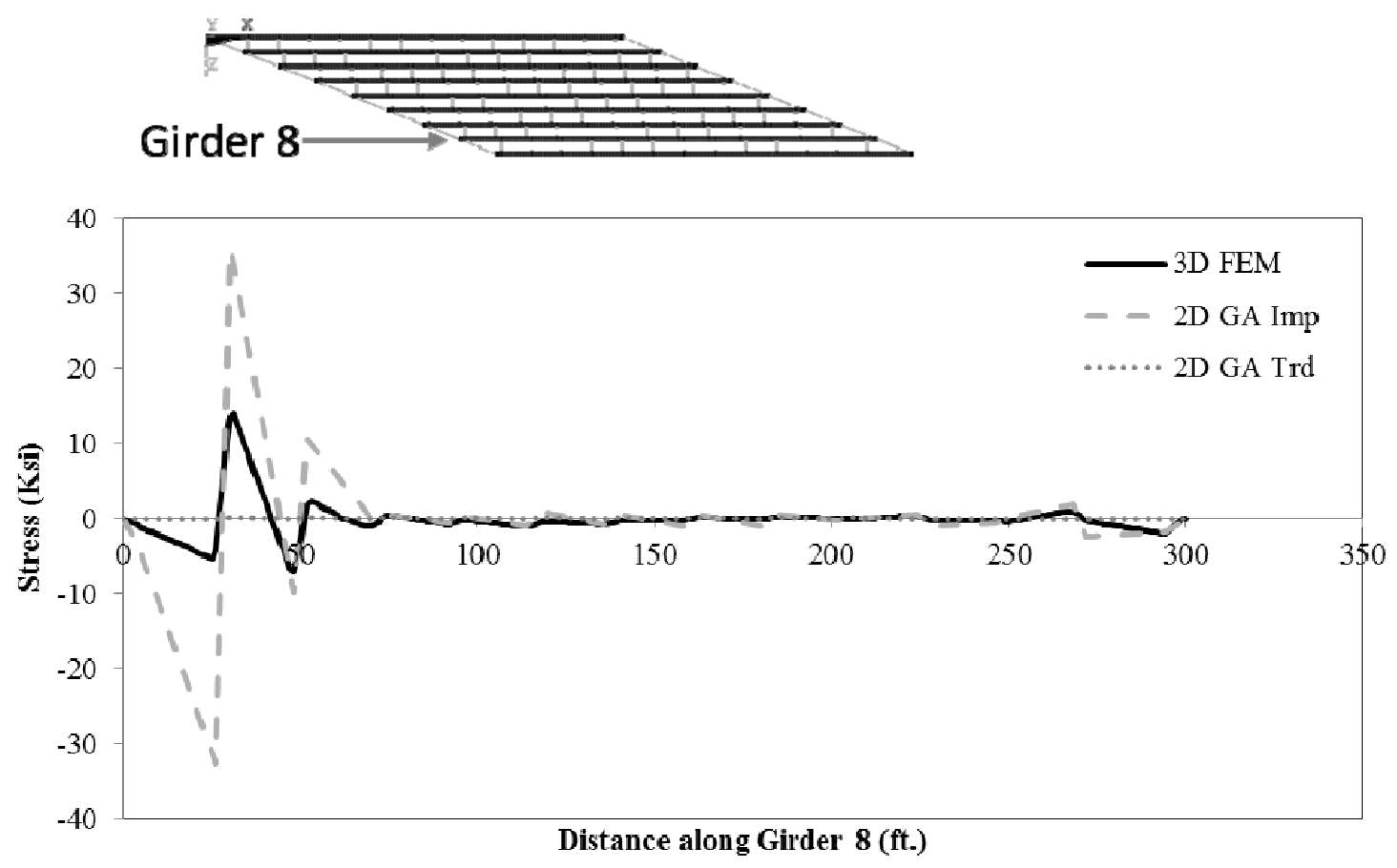

Figure 3.7: Comparison of flange lateral bending stress calculated by different analysis method in Girder 8 of Bridge $A$
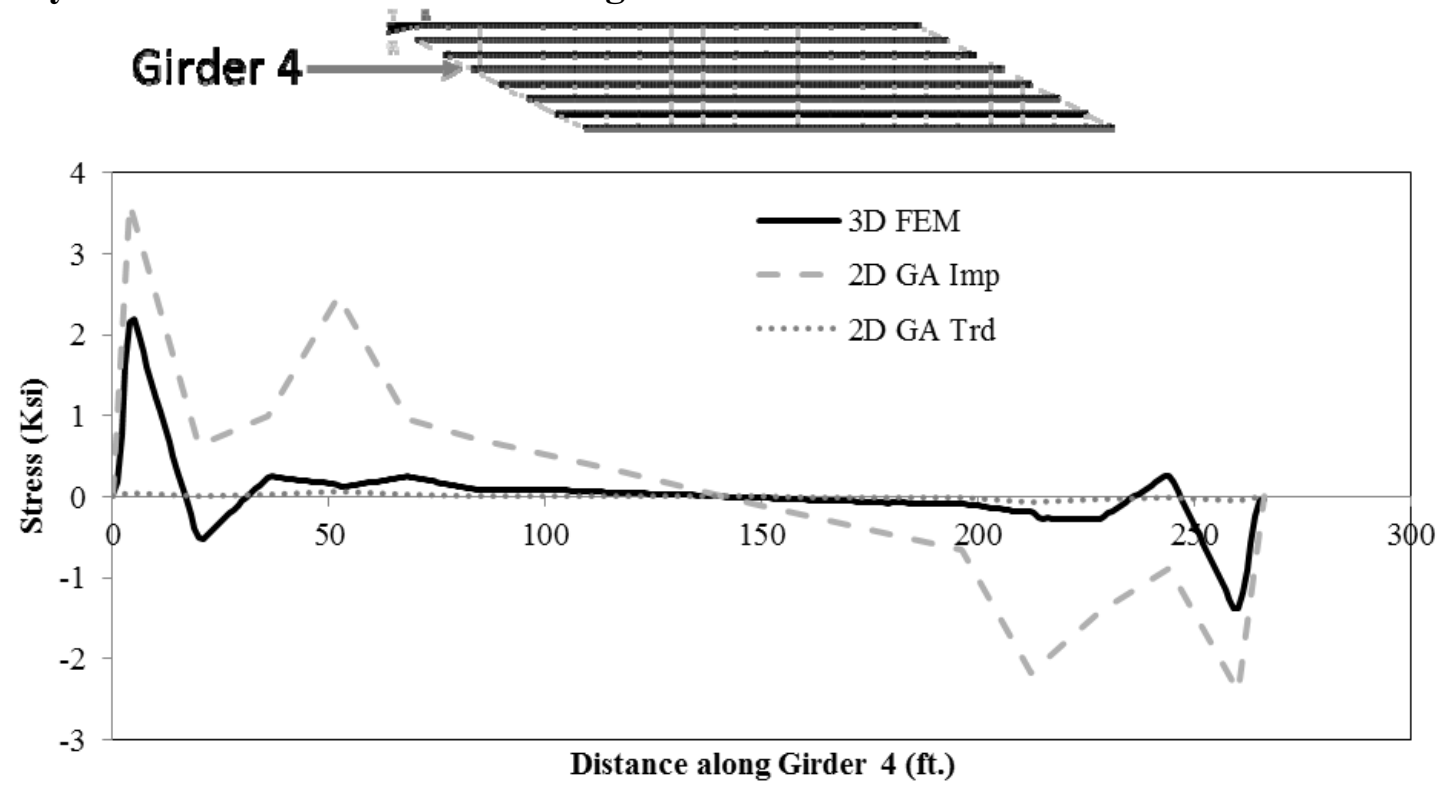

Figure 3.8: Comparison of flange lateral bending stress calculated by different analysis methods in Girder 4 of Bridge B 


\subsubsection{Cross-frame Forces}

Cross-frame forces can be obtained from the 2D GA by multiplying displacements at connections of a cross-frame to girders to the axial stiffness of members in the cross-frame. Detail of this approach is given in NCHRP 725 [9]. Cross-frame forces from the 3D FEM analysis can be directly obtained from forces in link elements used for modeling cross-frame members. Comparison of cross-frame forces obtained from different methods of analysis is done and is shown for the top chord of cross-frames in bay 1 of Bridge A in Figure 3.9 and for the top chord of cross-frames in bay 4 of Bridge B in Figure 3.10 for the Erected Fit detailing method at the TDL stage.

It can be observed that the difference between cross-frame forces obtained from different methods of analysis is significant. Comparison also indicates that cross-frame forces are highest for the improved 2D grid analysis and lowest for the 3D FEM analysis in case of Bridge A. The improved 2D-grid analysis significantly over-estimates crossframe forces compared to the 3D FEM analysis. Cross-frame forces evaluated from the traditional 2D-grid analysis are essentially zero, due to the gross underestimation of the girder torsional stiffness in the traditional 2D-grid methods for Bridge A. The difference in cross-frame forces for Bridge B is not very significant. The results of a broad range of analyses on the different bridges demonstrate that the improved 2-D grid analysis is

sufficient to calculate the cross-frame forces. The results from the improved 2D-grid analysis are generally accurate and conservative compared to results from 3D FEM analysis. 


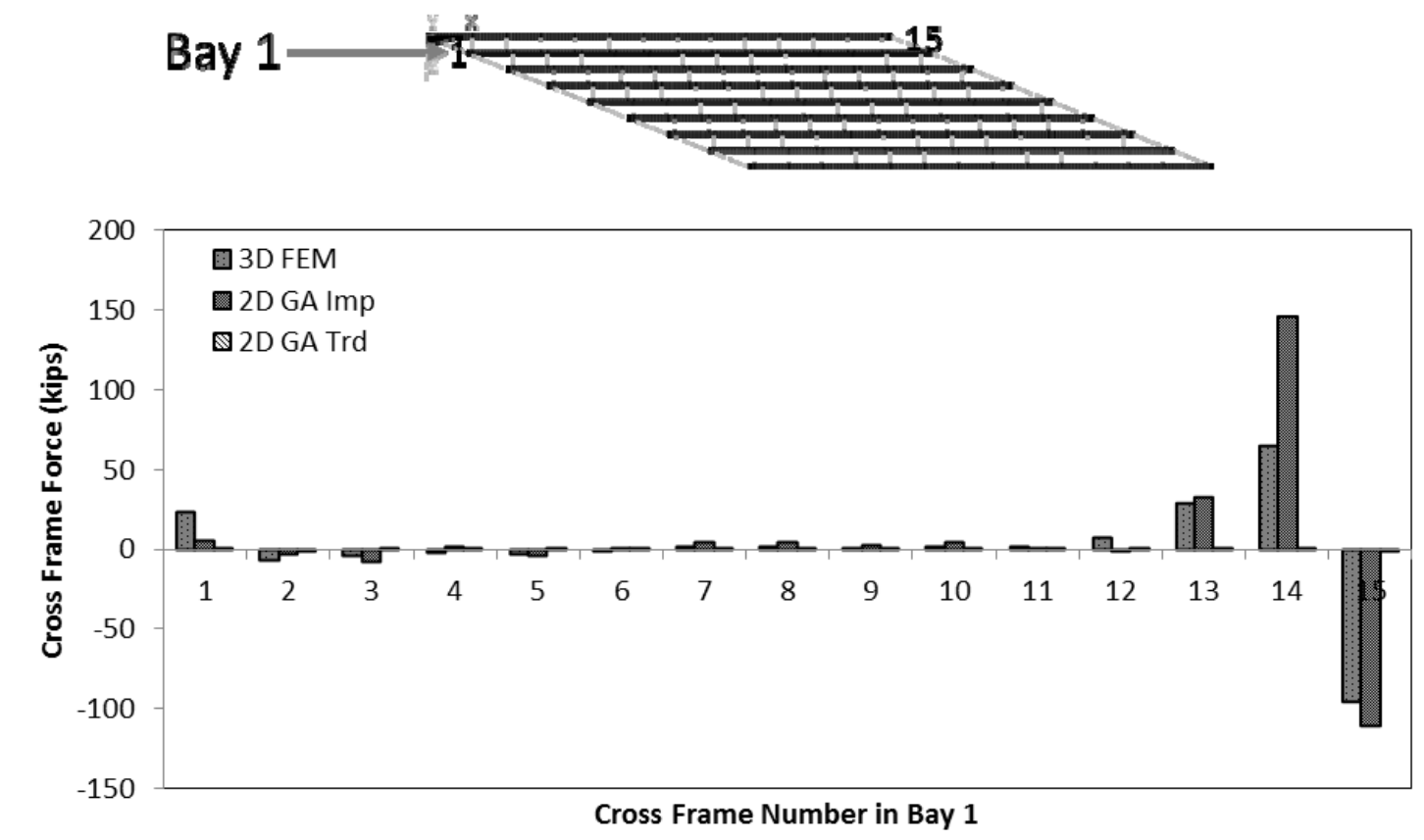

Figure 3.9: Comparison of cross-frame forces calculated by different analysis method for Bridge A

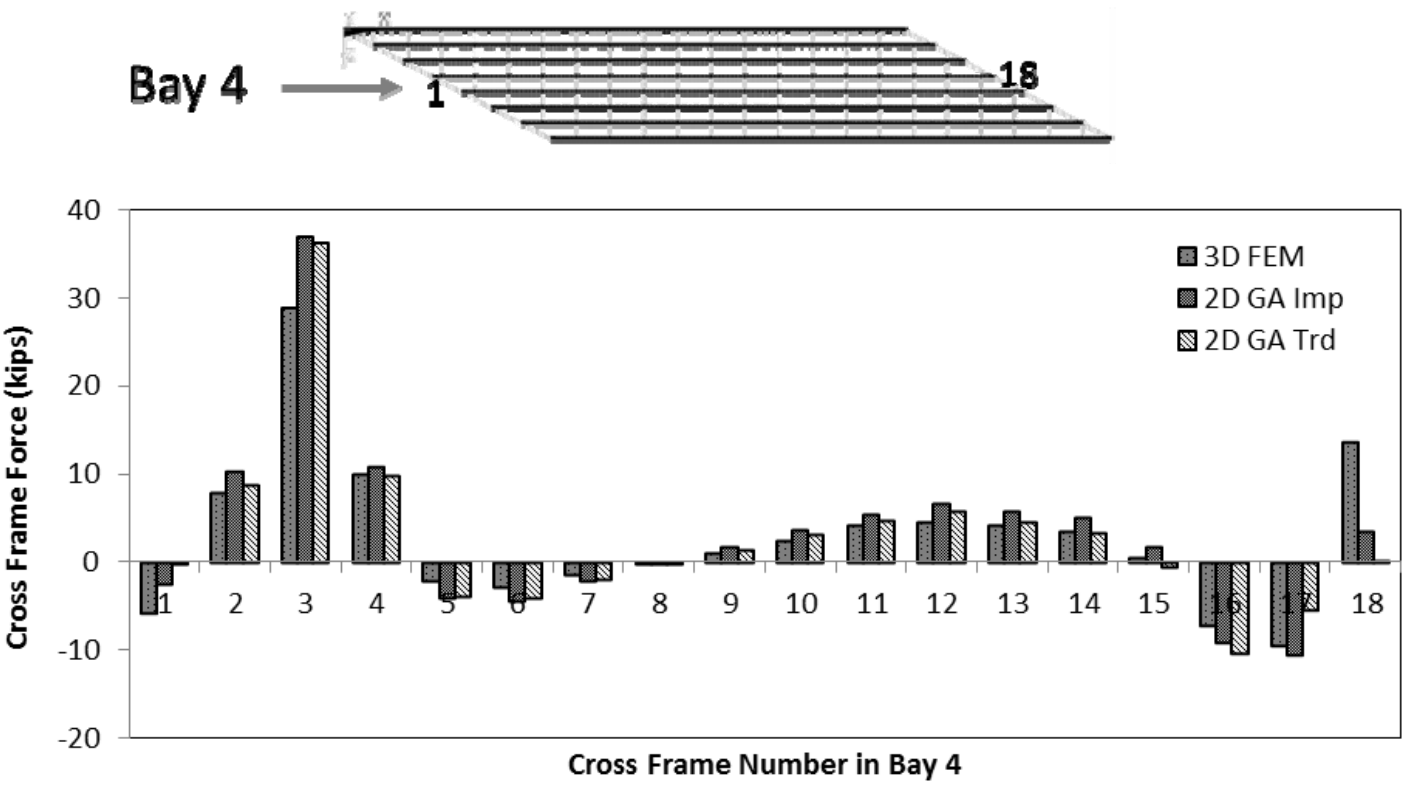

Figure 3.10: Comparison of cross-frame forces calculated by different analysis method for Bridge $B$ 


\subsection{Discussion of Results}

Lack-of-fit effects are significantly influenced by the torsional stiffness of girder for the straight skewed bridges having staggered cross-frames (Bridge A). With that being said, any increase or decrease in the estimated values of torsion stiffness may increase or decrease the lack-of-fit effects. The traditional 2D GA underestimates the torsional stiffness of the girders. This underestimation impacts cross-frame forces, component of vertical reaction, lateral reaction, and flange lateral bending stress significantly. The improved 2D GA analysis uses an equivalent torsional constant that gives good estimate of the torsional stiffness in most of cases. However, when staggered distance between two adjacent cross-frames in two adjacent bays become small (for example less than one fourth of the girder depth as in case of Bridge A), the equivalent torsional constant overestimates the torsional stiffness of the small segment of girder between the two adjacent cross-frames. The effect of this overestimation is more pronounce in case of component of reaction due to lack-of-fit, cross-frame forces, and flange lateral bending stress. The component of reaction due to lack-of-fit, cross-frame forces, and flange lateral bending stress are overestimated by the improved 2D GA due to overestimation of the torsional stiffness of the small girder segments between staggered cross-frames. Therefore, it is recommended to avoid small staggered distances while using the improved 2D GA. There are good chances that results from the improved 2D GA blow up in case a very small staggered distance is entered in the program by mistake.

In case of a straight skewed bridge with cross-frames arranged in contiguous lines, such as, Bridge $\mathrm{B}$ and Bridge $\mathrm{C}$, the only lack-of-fit effect sensitive to torsional stiffness of the girders is flange lateral bending stress. The improved 2D GA also 
overestimates the lack-of-fit effects for Bridge $\mathrm{B}$ and Bridge $\mathrm{C}$; however, the overestimation is not significant.

Table 3.1: Performance of traditional and improved 2D GA

\begin{tabular}{|l|c|c|c|c|}
\hline \multirow{2}{*}{ Lack-of-fit effect } & \multicolumn{2}{|c|}{ Staggered cross-frames } & \multicolumn{2}{c|}{ Contiguous cross-frames } \\
\cline { 2 - 5 } & $\begin{array}{c}\text { Traditional } \\
\text { 2D GA }\end{array}$ & $\begin{array}{c}\text { Improved } \\
\text { 2D GA }\end{array}$ & $\begin{array}{c}\text { Traditional } \\
\text { 2D GA }\end{array}$ & $\begin{array}{c}\text { Improved } \\
\text { 2D GA }\end{array}$ \\
\hline Girder Layovers & Poor & Ok & Ok & Ok \\
\hline Vertical Reaction & Poor & Ok & Ok & Ok \\
\hline Cross-frame forces & Poor & Ok & Ok & Ok \\
\hline Vertical Deflection & Poor & Ok & Ok & Ok \\
\hline Flange lateral bending stress & Poor & Ok & Poor & Ok \\
\hline
\end{tabular}

It is important to remember that these comparisons are made between two analysis methods and not with experimentally measured responses. As long as an analysis method gives a reasonable trend with conservative estimates of a response, it can be utilized for the design purpose. It is general practice to simplify the structure while carrying out the analysis for design purpose. Therefore, the results of this simplified analysis might not match perfectly with the measured response of the structure; however, can be used for design purpose.

\subsection{Methods of Analysis for the Final Fit Detailing Method}

The main objective of this section is to introduce the use of simplified and existing 3D FEM analysis for the Final Fit detailing method. Following methods analysis can be used to calculate lack-of-fit effects for the Final Fit detailing method at the SDL stage:

1. Reversing the $2 \mathrm{D}$ GA results for the Erected Fit 
2. 3D FEM analysis using initial strains

3. 3D FEM analysis using Dead and Live cross-frames

The first method in the above list is a simplified method, introduced in this research because of the main conclusion made in chapter 2 . The second method is a $3 \mathrm{D}$ FEM analysis method introduced in NCHRP 725 [9]. The third method is another 3D FEM analysis method; however, much simpler compared to the second method and evaluate lack-of-fit effects with same accuracy. Detailed description of each method is provided in the following sections.

\subsubsection{Reversing 2D GA Results for the Erected Fit}

Numerical studies carried out in chapter 2 has shown that the lack-of-fit effects for the Final Fit detailing method at the SDL stage are equal and opposite to the lack-offit effects for the Erected Fit detailing method at the TDL stage. Lack-of-fit effects for the Erected Fit detailing method at the TDL stage can be obtained from the improved 2D grid analysis and reversing their sign gives lack-of-fit effects for the Final Fit detailing method at the SDL stage.

\subsubsection{D FEM analysis Using Initial Strains}

In this method different components of bridge are modeled as described in section 2.4 and section 3.1.3. However, the only difference is that for the Final Fit detailing method the cross-frames do not fit between their connections to girders at the SDL stage. This lack-of-fit at the SDL stage is modeled by using initial strains in cross-frame members.

These initial strains are calculated using different configurations for intermediate cross-frames perpendicular to girder web and cross-frames parallel to skewed support. 
The configurations of cross-frames and girders to calculate initial strains are shown in Figure 3.11 for cross-frames perpendicular to webs and in Figure 3.12 for a cross-frame parallel to skew. Configuration 1 represents a real situation in which cross-frames do not fit between their connections to girders at the SDL stage for the Final Fit detailing method. Configuration 2 represents an imaginary condition in which cross-frame members are deformed (stretched or shortened) to make connections that were not made in the configuration 1. Configuration 2 is an imaginary high-energy configuration of the system lacking the equilibrium. Once the system is allowed to establish the equilibrium, it attains its lowest energy state. After the equilibrium is established, the system has a real configuration of the steel framing for the Final Fit detailing method at the SDL stage after connecting cross-frames to girders.

For any orientation of cross-frames, parallel to a skew or perpendicular to a web, the initial strain $\left(\varepsilon_{\text {Initial }}\right)$ in any cross-frame member can be calculated by the following formula:

$$
\varepsilon_{\text {Initial }}=\frac{L_{1}-L_{2}}{L_{2}}
$$

Where, $L_{1}$ is the length of a cross-frame member in configuration 1 , and $L_{2}$ is the length of a cross-frame member in configuration 2 . The two configurations of the crossframes are shown in Figure 3.11, for a cross-frame that is perpendicular to webs, and are shown in Figure 3.12, for cross-frames parallel to a skew.

The length of members of a cross-frame, perpendicular to girder web, in configuration 1 (shown in Figure 3.11) can be calculated as follows: 


$$
\begin{gathered}
L_{T C_{1}}=L_{B C_{1}}=S \\
L_{D 1_{1}}=L_{D 2_{1}}=\sqrt{S^{2}+h_{b}^{2}}
\end{gathered}
$$

Where $L_{T C_{1}}, L_{B C_{1}}, L_{D 1_{1}}, L_{D 2_{1}}$ are lengths of top chord (TC), bottom chord (BC), diagonal 1 (D1) and diagonal 2 (D2) members of the cross-frame in configuration $1, S$ is the spacing between girders, and $h_{b}$ is the height of bracing.

Similarly, the length of the cross-frame members that are perpendicular to webs in configuration 2 of Figure 3.11 can be calculated as follows:

$$
\begin{aligned}
& L_{T C_{2}}=L_{B C_{2}}=\sqrt{S^{2}+\Delta^{2}} \\
& L_{D 1_{2}}=\sqrt{S^{2}+\left(h_{b}-\Delta\right)^{2}} \\
& L_{D 2_{2}}=\sqrt{S^{2}+\left(h_{b}+\Delta\right)^{2}}
\end{aligned}
$$

Where, $L_{T C_{2}}, L_{B C_{2}}, L_{D 1_{2}}, L_{D_{2}}$ are lengths of top chord (TC), bottom chord (BC), diagonal 1 (D1) and diagonal 2 (D2) members of the cross-frame in configuration 2.

The difference in elevation of the girders' section to be connected by the crossframe, $\Delta$, is obtained from the concrete dead load camber calculated from the line girder analysis (LGA) or the isolated girder analysis (IGA) in this study. $\Delta$ can also obtained from the concrete dead load camber calculated from the vertical deflection of the system of girders and cross-frames attached together. In NCHRP 725 [9], $\Delta$ for dead loads was obtained from the vertical deflection of the system of girders and cross-frames attached together. It is important to mention that $\Delta$ calculated from the dead deflection of isolated 
girders is different from $\Delta$ calculated from the dead deflection of the system of girders and cross-frame attached together. The difference between the two depends on the bridge geometry, size of different components and the magnitude of dead loads. In some case such as, Bridge A, this difference can be significant and results in significant difference in calculated lack-of-fit effects. Calculation of $\Delta$ from the vertical deflection of isolated girders in this study is justified in chapter 2 of this dissertation.

It should be noted that $\Delta$ is introduced here is the difference in elevation of girders at the SDL stage for simplicity. The difference in elevations of girders also come from the bridge cross slope that is built into the cross-frames regardless of the detailing method. Therefore, to be more precise, $\Delta$ is the difference in elevation of cross-frames and their connections to girders due to detailing method as shown in Figure 3.11.

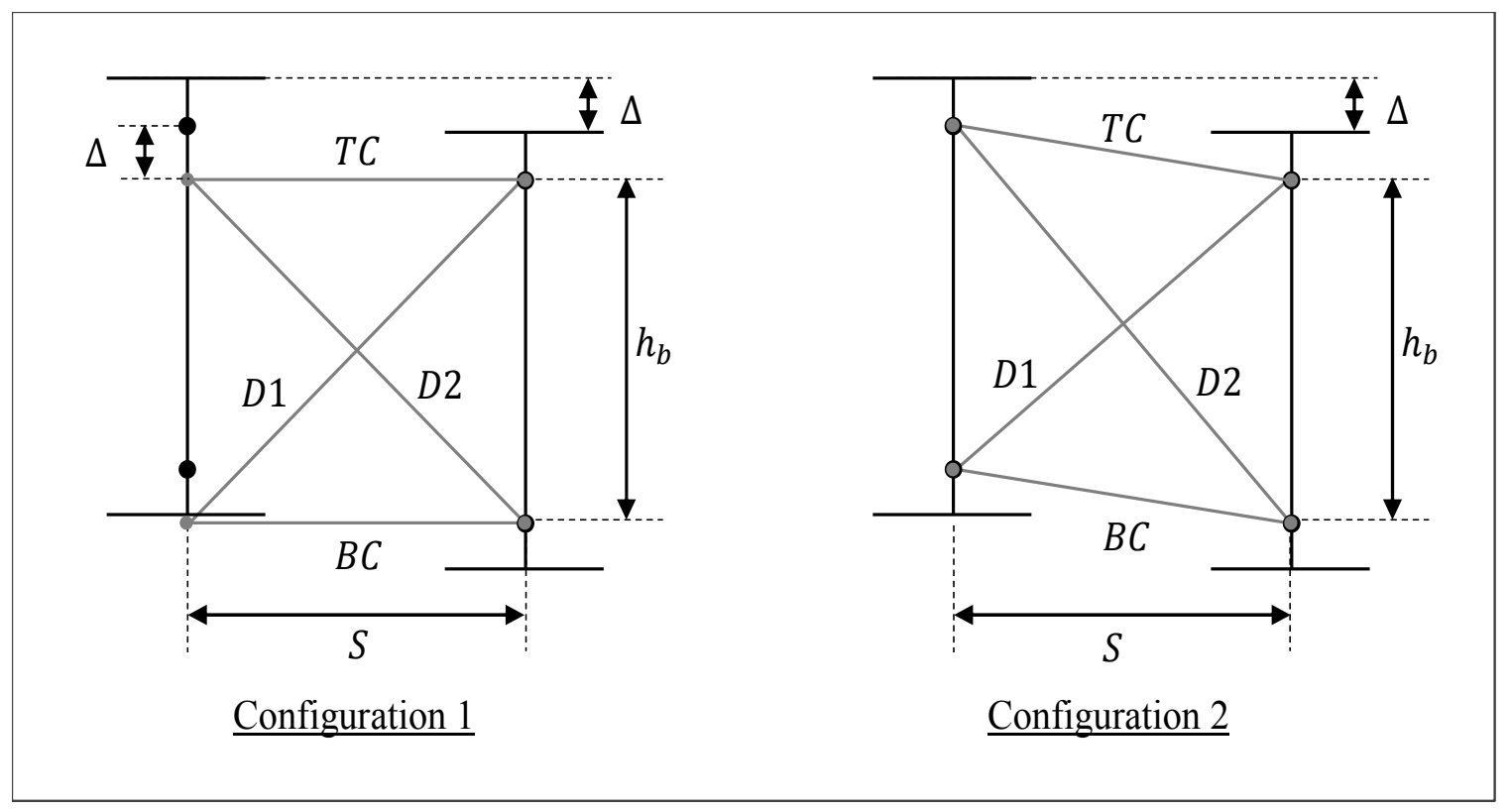

Figure 3.11: Configurations to calculate initial strain in the cross-frames that are perpendicular to girder web 
Lack-of-fit in the cross-frames that are parallel to skewed supports, occurs due to major axis bending rotation of the girder section as shown in Figure 3.12. Figure 3.12 illustrates the configuration of cross-frames parallel to the skewed support at the bearing lines. The intermediate cross-frames parallel to skew also have similar configurations. Configuration 1 in Figure 3.12 shows that the cross-frame does not fit between its connections to girders due to major axis bending rotation $(\phi)$ of girders' ends. In configuration 2, the cross-frame is deformed to make the connections as described previously for cross-frames perpendicular to the girder web.

The length of members of a cross-frame, parallel to the skew support, in configuration 1 (shown in Figure 3.12) can be calculated as follows:

$$
\begin{gathered}
L_{T C_{1}}=L_{B C_{1}}=\sqrt{\Delta_{x}^{2}+S^{2}} \\
L_{D 1_{1}}=L_{D 2_{1}}=\sqrt{\Delta_{x}^{2}+h_{b}^{2}+S^{2}}
\end{gathered}
$$

Neglecting the displacement in Y-direction of connections and taking $\sin \theta \cong \theta$ it can be shown that the length of cross-frame members in configuration 2 can be calculated as follows:

$$
\begin{gathered}
L_{T C_{2}}=L_{B C_{2}}=\sqrt{\Delta_{x}^{2}+S^{2}} \\
L_{D 1_{2}}=\sqrt{\left(\Delta_{x}-\phi \cdot h_{b}\right)^{2}+h_{b}^{2}+S^{2}} \\
L_{D 2_{2}}=\sqrt{\left(\Delta_{x}+\phi \cdot h_{b}\right)^{2}+h_{b}^{2}+S^{2}}
\end{gathered}
$$

And

$$
\Delta_{x}=S \times \tan \theta
$$


Where, $\theta$ is the skew angle and $\phi$ is the major axis bending rotation of isolated girders due to concrete dead load at the location of the cross-frame. $\phi$ is positive (counter clockwise) for the situation shown in Figure 3.12. $\phi$ is negative (clockwise) for the other end of the girders shown in Figure 3.12.

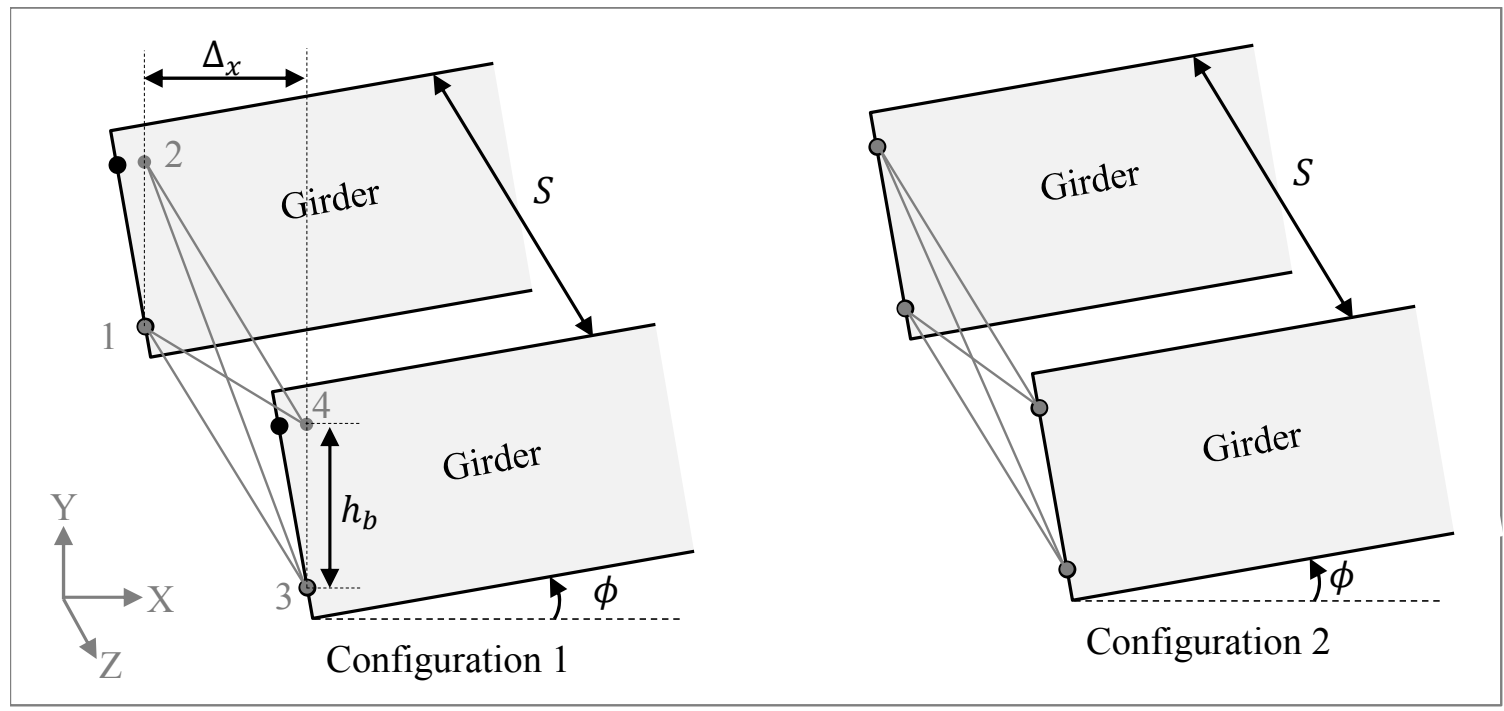

Figure 3.12: Configurations to calculate initial strain in the cross-frames that are parallel to skew

In order to get the SDL configuration for the Final Fit detailing method, complete model of the bridge is built with cross-frames attached to girders. A particular value of initial strain is assigned to each cross-frame member that can be calculated based on location and orientation of the cross-frame and type of the cross-frame member as described above. Once initial strains are assigned to all the cross-frame members, the static analysis is run without applying any external load. In this static analysis the cross- 
frame members expand or contract depending on the magnitude of the initial strain and establish equilibrium with girders. Once equilibrium is established, the steel framing of bridge achieves its stable lowest possible energy configuration. The geometry of the bridge obtained after the equilibrium is established represents the bridge's geometry at the SDL stage for the Final Fit detailing method.

\subsubsection{D FEM analysis Using Dead and Live Cross-frames}

Lack-of-fit of cross-frames to their connection to girder at Steel Dead Load (SDL) stage for the Final Fit detailing method can also be simulated by using birth and death option for the cross-frame elements. Detail description of birth and death elements is provided in ANSYS Advance Analysis Techniques Guide [36] and a detailed example with both analytical and birth and death solution is provided in ANSYS Mechanical APDL Verification Manual [37] and in Appendix E of this dissertation. A brief summary of using birth and death technique in 3D FEM analysis is provided here.

When an element is killed or made dead, its stiffness is reduced by a sever reduction factor (1.0E-06 by default). Element loads associated with the dead element are zeroed out of the load vector. When an element is born or made live, its stiffness and loads are returned to their full original values.

In the Final Fit detailing method, cross-frames fit between their connections to girders after application of the concrete dead load. Therefore, in this analysis the concrete dead load is applied on the girders to deflect the girders to a position in which crossframes fit between their connections to girders. Once girders are deflected by the concrete 
dead load, the cross-frames are made alive. After that, the concrete dead load is removed to get the SDL responses for the Final Fit detailing method.

It is two steps FEA after completing the bridge geometry with cross-frames attached as described follows:

Step 1: All the cross-frame elements are killed (using EKILL command in ANSYS) and the concrete dead load is applied as shown in Figure 3.13.

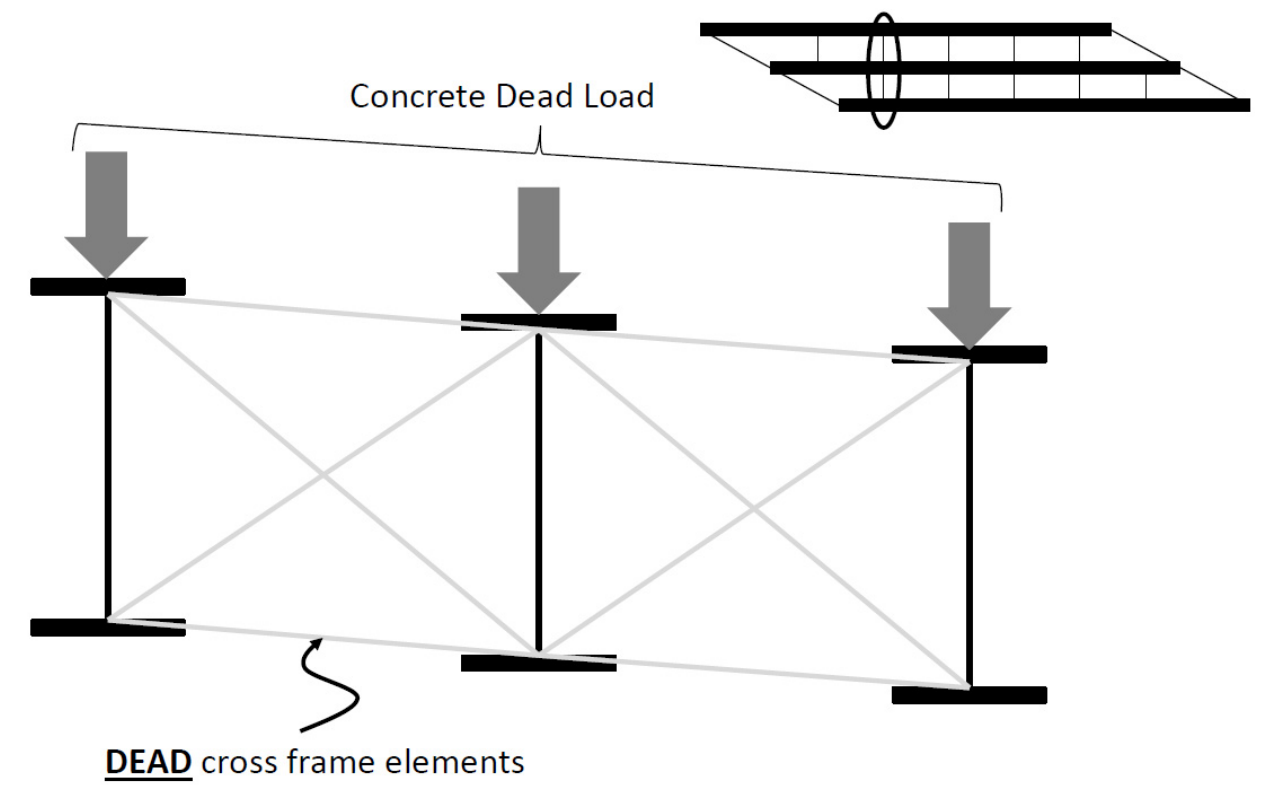

Figure 3.13: Application of the concrete dead load on girders after killing crossframe elements

Step 2: After the concrete dead load has deflected the girders, all the cross-frame elements are made alive (using EALIVE command in ANSYS) and the concrete dead load is removed (made zero) as shown in Figure 3.14. 


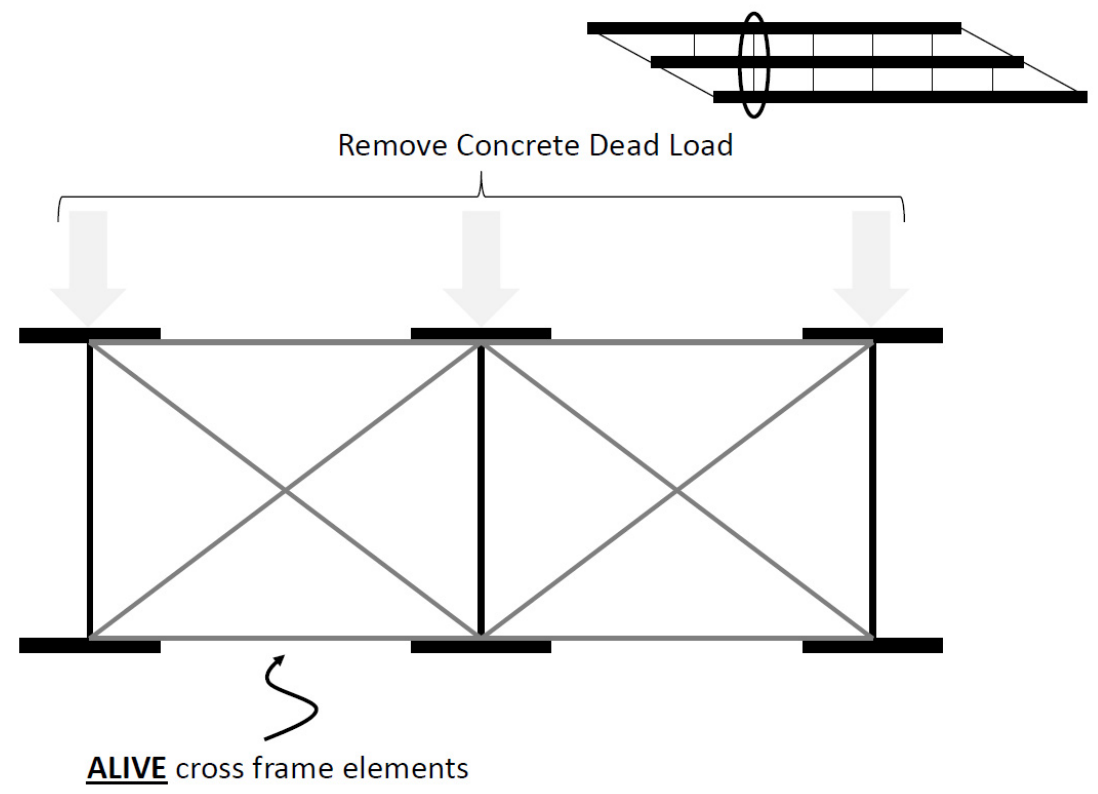

Figure 3.14: Removal of the concrete dead load from girders after making crossframe elements alive

At the completion of step 2, the SDL configuration of bridge framing is obtained for the Final Fit detailing method. The SDL configuration of a bridge can also be obtained by applying the negative concrete dead load to the system of girders and crossframes. However, this would result in major axis bending moments and stresses in the girders due to the negative concrete dead load which is not true in reality. Application and removal of the concrete dead load avoid creating major axis bending moments and stresses in the girder and represents a real situation.

It is also worth noting that the method of dead and live cross-frames does not involve laborious calculation of the initial strain for every single cross-frame member and gives the same results as the method of initial strains. The detailed comparison of different responses obtained from different method of analysis is done in section 4.2. 


\subsection{Comparison of Different Methods of Analysis for the Final Fit Detailing Method}

Different lack-of-fit effects such as, girder layovers, the component of the vertical deflection due to lack-of-fit $\left(\mathrm{D}_{\mathrm{Y} 2}\right)$, the component of vertical reaction due to lack-of-fit $\left(\mathrm{R}_{\mathrm{Y} 2}\right)$, the flange lateral bending stress $\left(f_{l}\right)$, and cross-frame forces are compared for different methods of analysis in Figure 3.15 to Figure 3.19for the Final Fit detailing method at the SDL stage.

3D FEM analysis using initial strains and 3D FEM analysis using dead and live cross-frame element gives almost the same estimates of different lack-of-fit effects for all the straight skewed bridges studied as a part of this research. This fact further endorses the mechanism explained in section 2.6 of this dissertation. When cross-frame elements are killed and the concrete dead load is applied, the girders deflect as isolated girders. After the girders are deflected due to the concrete dead load, cross-frames are activated. Notice, cross-frames have zero forces as they fit perfectly between their connections to girders at the TDL stage. Once the concrete dead load is removed, the girders go back to their original position; however, due to presence of active cross-frames girders do not end up at the same position they started with. This difference in position is created by lack-offit of cross-frames between their connections to girders and should not be taken into account while calculating the initial strains for modeling the lack-of-fit.

Reasonable estimates of lack-of-fit effects are obtained by reversing improved 2D grid analysis for both bridges. The overestimation of responses by reversing the improved 2D GA results for the Final Fit detailing method is similar to the overestimation of 
responses by the improved 2D GA for the Erected Fit detailing method and is discussed in detail in section 3.3.

(Note: Figure 3.15 through Figure 3.19 use 3D FEM initial strains to connote 3D FEM analysis using initial strains, 3D FEM Dead \& Live to connote 3D FEM analysis using dead and live cross frame elements, and 2D GA reversed to connote improved 2D grid analysis with reverse sign of results.)


Figure 3.15: Comparison of girder layovers calculated by different analysis methods 


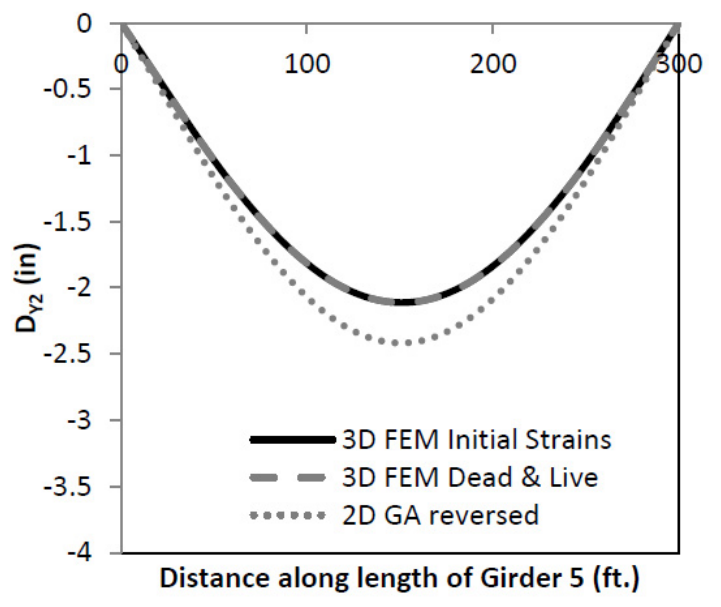

Bridge A

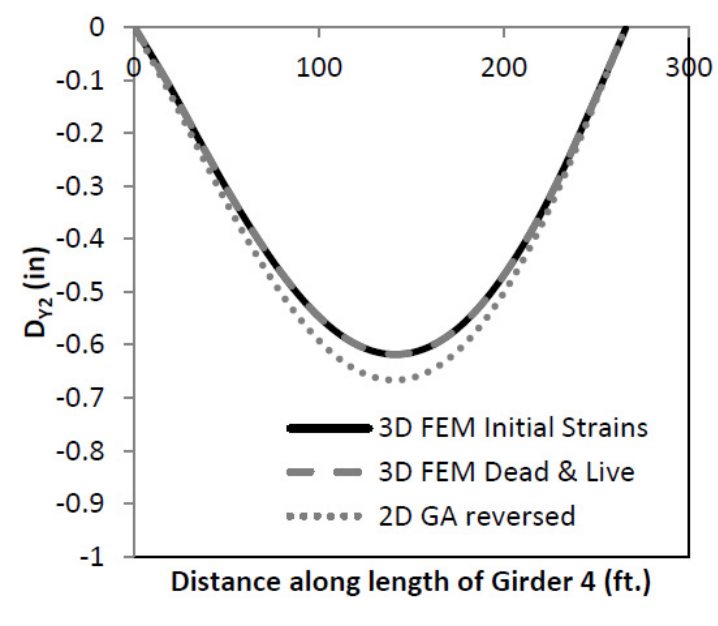

Bridge $B$

Figure 3.16: Comparison of the component of the vertical deflection due to lack-offit $\left(D_{\mathrm{Y} 2}\right)$ calculated by different analysis methods

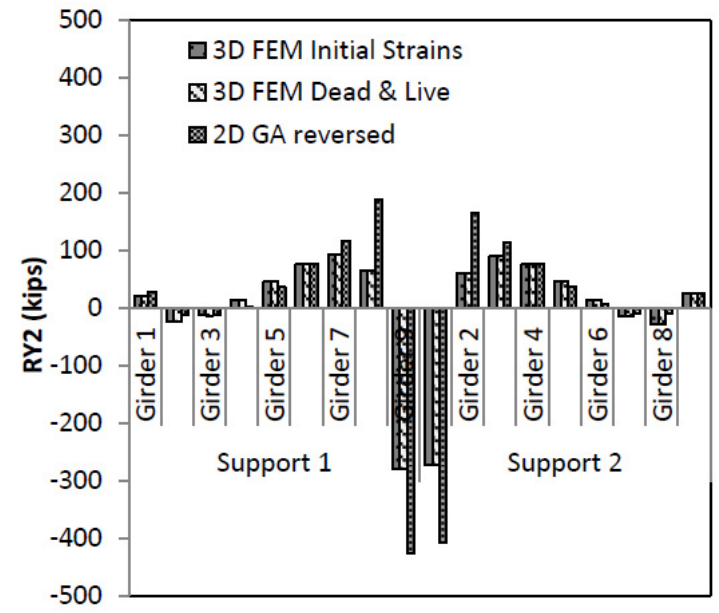

Bridge A



Bridge $B$

Figure 3.17: Comparison of the vertical reactions due to lack-of-fit $\left(\mathbf{R}_{\mathrm{Y} 2}\right)$ calculated by different analysis methods 


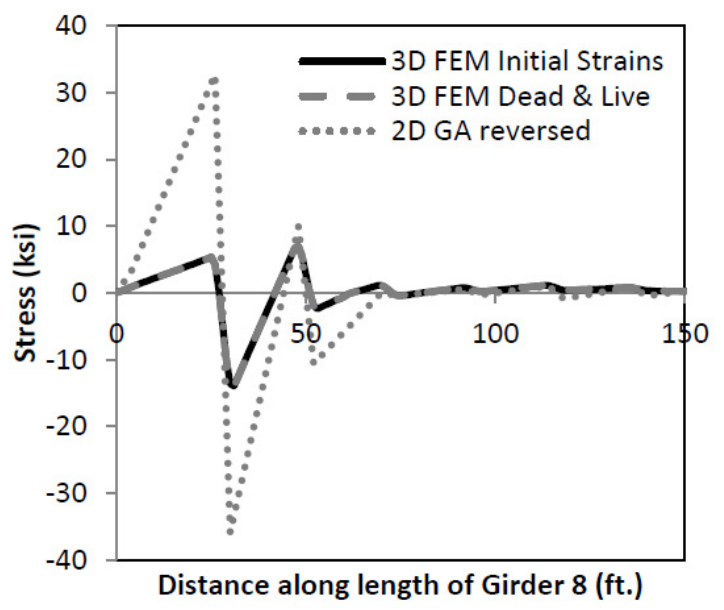

Bridge $\mathrm{A}$

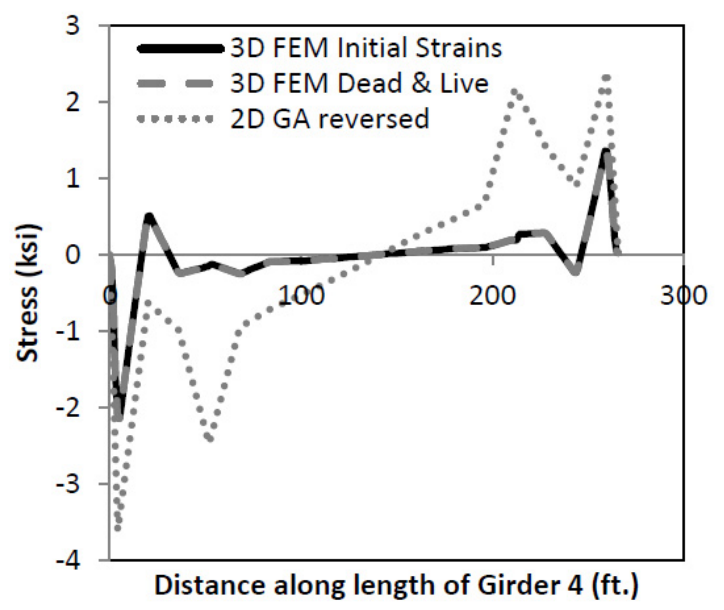

Bridge $B$

Figure 3.18: Comparison of the flange lateral bending stress calculated by different analysis methods


Figure 3.19: Comparison of cross-frame forces calculated by different analysis methods 


\subsection{Summary}

This chapter discusses different methods of analysis that can be used for calculating structural responses affected by different detailing methods. Different methods of analysis available in literature are discussed and compared for both Erected Fit and Final Fit detailing methods.

It is important to note that NCHRP 725 [9] has used 2D grid analysis for no load fit detailing method only. A concept of using 2D grid analysis for steel dead load fit (Erected Fit) and total dead load fit (Final Fit) is described in NCHRP 725 [9]; however, numerical results using this concept were not shown. Another contribution made by this chapter is to caution the designer for using the improved 2D GA for the staggered crossframes having small stagger distance. The improved 2D GA might give high estimates of lack-of-fit effects depending on the staggered distance.

The main conclusion of this chapter is that improved 2D grid analysis can be used to calculate the lack-of-fit effects for different detailing methods at different loading stages. Further, a simplified 3D FEM analysis is introduced to get the state of the skewed bridge frame, detailed with the dead load detailing method, at different loading stages. This 3D FEM analysis does not require tedious initial strain calculations and give the same results as obtained from method of initial strains.

For the erected fit detailing method, performance of improved and traditional 2D GA is different for different framing options as follows:

- For bridges with contiguous cross frames, traditional 2D GA gives reasonable estimates of all responses except for flange lateral bending stress and improved 2D GA gives reasonable estimates of all responses. 
- For bridges with staggered cross frame, traditional 2D GA gives erroneous estimates of all the responses and improved 2D GA gives reasonable estimates of all responses. However, when stagger distance is small, $J_{e q}$ in improved 2D GA has very high value resulting in overestimation of lack-of-fit effects.

For the Final Fit detailing method, the cross-frames are connected to girders during the erection of steel bridge by using fit-up forces. Methods of analysis, both the 3D FEM analysis and the simplified 2D GA, are not available to estimate these fit-up forces. Next chapter describes these fit-up forces in detail and also provide both 3D FEM analysis and simplified 2D GA to estimate these forces. 


\section{METHODS OF CALCULATING FIT-UP FORCES}

In the Final Fit detailing method, the web of different girders is out-of-plumb at the completion of the erection and before casting of the concrete deck or the steel dead load (SDL) stage and ideally deflect into the plumb position after casting the deck or the total dead load (TDL) stage as shown in Figure 4.1. Some owners, erectors, fabricators and detailers prefer Final Fit detailing method because of the plumb girder web at the final permanent loading stage (TDL stage). It should be noted that for the final fit, the webs may not be perfectly plumb at the TDL stage. This can be due to uncertainties in the actual restraints at bearings, or differential curing of the concrete bridge deck, as well as several other factors.

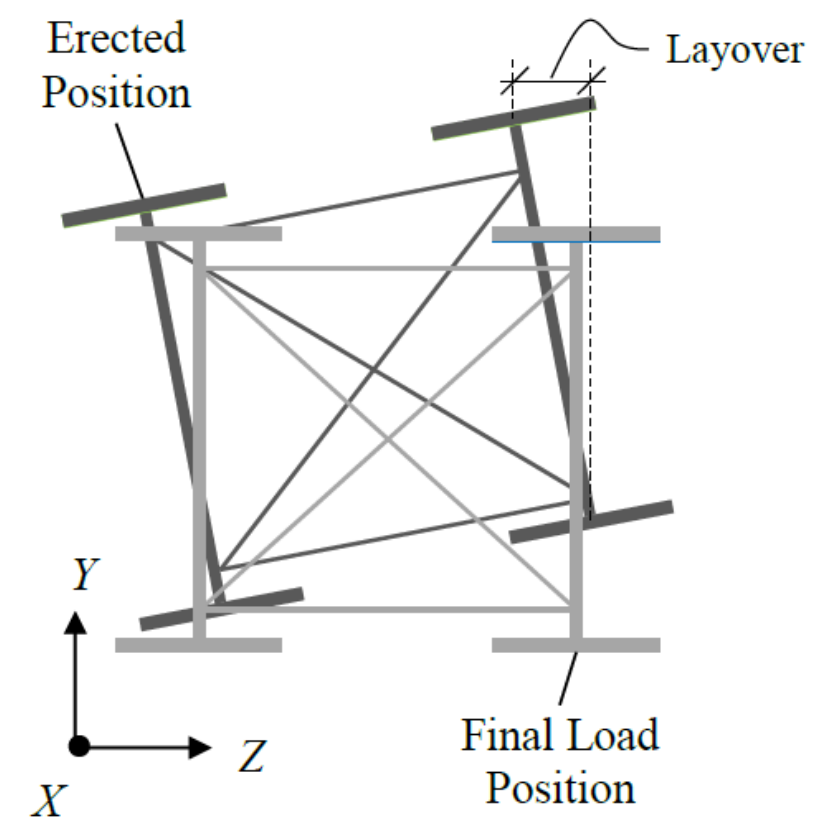

\section{Figure 4.1: Final Fit detailing methods}

In the Final Fit detailing method, cross-frames are fabricated to fit between their connections to girders at the TDL stage and therefore, these cross-frames do not fit 
between their connections to girders at the SDL stage. In order to connect the crossframes to girders at the SDL stage, erector needs to apply a force, generally referred as fit-up force, to connect or fit the cross-frame between their connections to girders. It is important to know the magnitude of the fit-up force, required to fit the cross-frame between its connections to girders, beforehand to make arrangements for the application of the fit-up force.

Before discussing the fit-up forces in detail, it is important to understand the lackof-fit of cross-frame, detailed with the Final Fit detailing method, between their connections to girders during the erection of skewed bridges.

\subsection{Lack-Of-Fit in Skewed Bridges}

Lack-of-fit refers to the fact that cross-frames do not fit between their connections to girders at a particular loading stage in skewed bridges. Lack-of-fit occurs at the Steel Dead Load (SDL) stage if the Final Fit detailing method is used and at the Total Dead Load (TDL) stage if the Erected Fit detailing method is used.

Figure 4.2 explains the lack-of-fit of cross-frames between their connections to girders for the Final Fit detailing method during the erection. It shows the framing plan of a skewed bridge and the concrete dead load cambers associated with each girder. The intermediate cross-frames in skewed bridges are typically perpendicular to the girder web, and connect the two adjacent girders at different elevations due to camber as shown in Figure 4.2.

It should be noted that the difference in the elevation of girders can come from both cross slope and girder cambers. However, the difference in elevation due to cross 
slope remains same at different loading stages and therefore does not contribute to lackof-fit. However, the difference in girders' elevations due to camber does contribute to lack-of-fit and is illustrated in Figure 4.2. The figure shows some of the detailing complexities that the differential camber produces with respect to the cross-frame detailing.

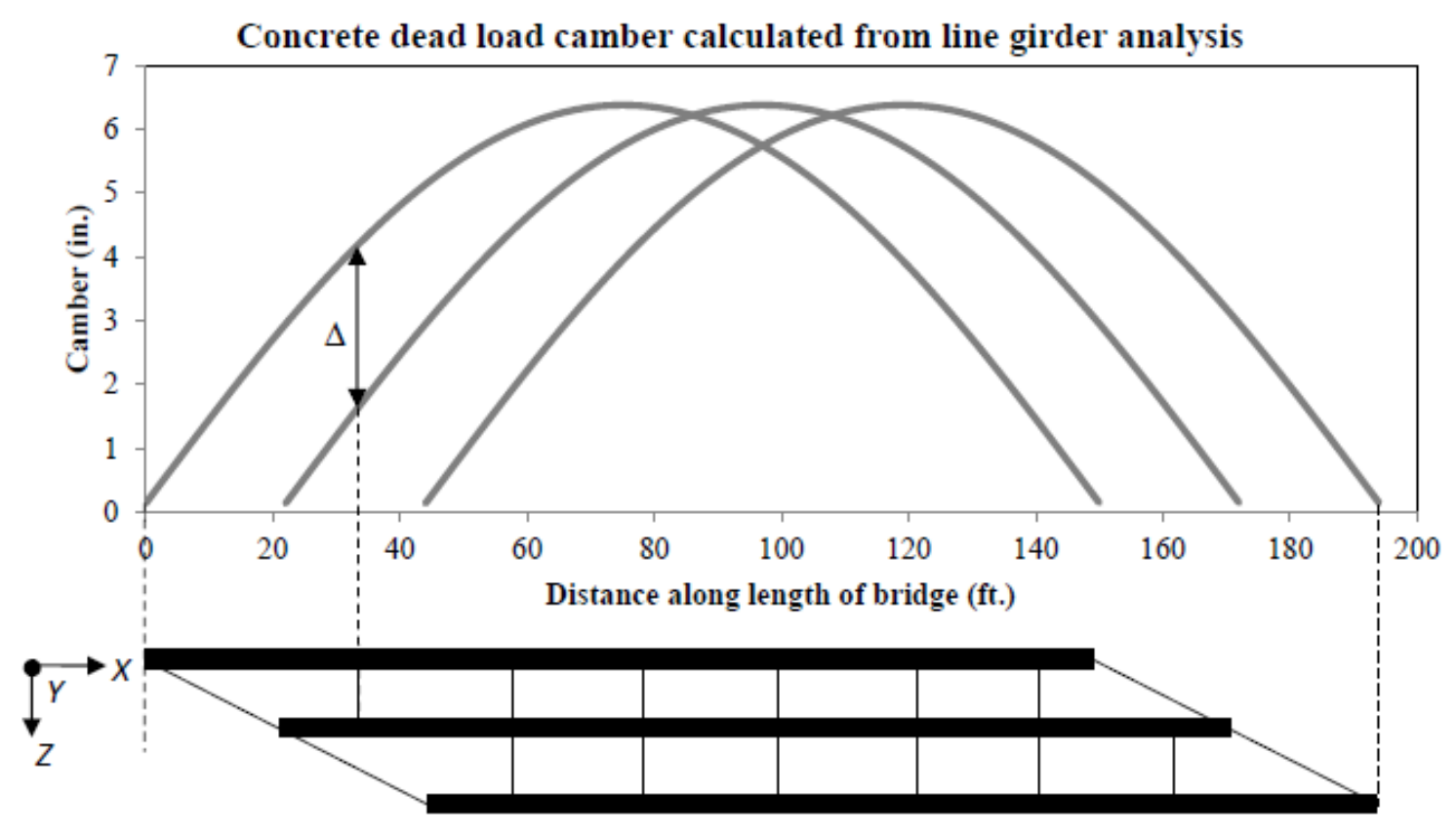

Figure 4.2: Differential camber in a skewed bridge

\subsection{Fit-Up Forces}

As explained earlier, in the Final Fit detailing method, the cross-frames are detailed to fit between their connections to girders at the total dead load (TDL) stage. Therefore, these cross-frames do not fit between the girders at the steel dead load (SDL) stage or during the erection of the steel frame. In order to fit the cross-frames, detailed with the Final Fit detailing method, between their connections to girders at the SDL stage 
or during the erection, a force is required to move the girders into a position where crossframes can be attached. The girders are both twisted and moved in vertical direction to fit the cross-frames between their connections to girders. This is accomplished by application of horizontal and vertical forces at the top and the bottom of the girders, henceforth called as fit-up forces. In theory, four fit-up forces are required to move a girder for attaching cross-frame; two vertical forces acting on the top and the bottom of girder $\left(F_{y}{ }^{T}\right.$ and $\left.F_{y}{ }^{B}\right)$ and two lateral forces acting on the top and the bottom of girder $\left(F_{z}{ }^{T}\right.$ and $F_{z}{ }^{B}$ ) as shown in Figure 4.3.

Knowledge of the fit-up forces will allow the bridge steel erector to make arrangements for application of the fit-up force. High fit-up forces are not desirable because these high forces can slow down the construction of skewed bridges.

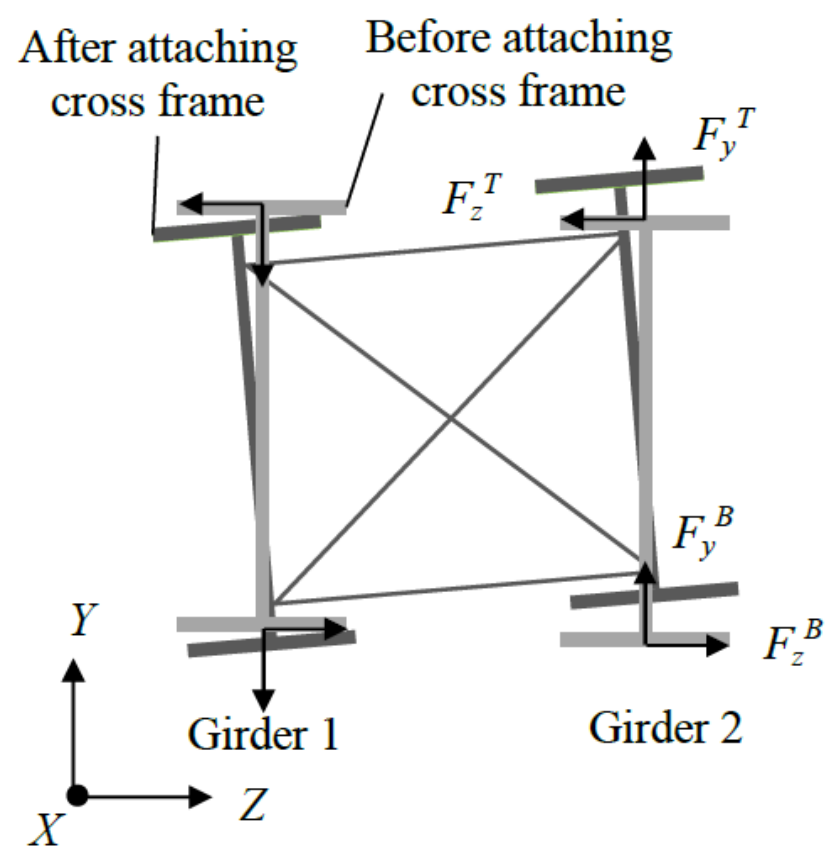

Figure 4.3: Fit-up forces required to attach the cross-frames to the girders 


\subsection{Proposed Methods of Calculating Fit-Up Forces}

Two methods are proposed to calculate the fit-up forces required to fit the crossframes, detailed with the Final Fit detailing method, between their connection girders during the erection. The followings are the two methods proposed for estimating the fitup forces:

- Cross-frame forces method

- 3D erection simulation method

Cross-frame forces method requires less computational effort and is less accurate compared to $3 \mathrm{D}$ erection simulation method that is more accurate but requires more computational effort.

The following sections provide the detail of each method.

\subsubsection{Cross-frame Forces Method}

Cross-frame forces at the SDL stage for the Final Fit detailing method are indicative of the fit-up forces. This is because the cross-frames are holding the girders into twisted positions or are responsible for lack-of-fit effects. Numerical studies carried out in chapter 2 of this dissertation has shown that cross-frame forces for the Final Fit detailing method at the SDL stage are equal and opposite to the cross-frame forces for the Erected Fit detailing method at the TDL stage. Therefore, cross-frame forces for the Final Fit detailing method at the SDL stage can be obtained by reversing the sign of the crossframe forces for the Erected Fit detailing method at the TDL stage as shown in chapter 3. Cross-frame forces for the Erected Fit detailing method at the TDL stage can be obtained from the improved 2D grid analysis, thereby avoiding the use of 3D FEM analysis. 
Cross-frame forces obtained from improved 2D GA they are resolved into vertical and lateral components at the connection points as shown in Figure 4.4 to get fit-up forces. The fit-up forces in vertical direction $\left(F_{y}\right)$ and lateral direction $\left(F_{z}\right)$ at a connection point can be calculated by resolving the cross-frame forces into vertical and lateral components at the connection. For example, following equations can be used to calculate fit-up forces at the top of Girder 2.

$$
\begin{gathered}
F_{y}{ }^{T}=-F_{D 1} \sin \theta \\
F_{z}{ }^{T}=-F_{T C}-F_{D 1} \cos \theta
\end{gathered}
$$

Where, $F_{B C}, F_{T C}, F_{D 1}$ and $F_{D 2}$ are forces in bottom chord, top chord, diagonal 1 and diagonal 2 members of the cross-frames. Although, erectors would be interested in only the magnitude of the maximum fit-up force, nonetheless, it is important to remember the local sign convention used for the cross-frame forces while calculating the direction of a fit-up force. Generally, the negative sign is used for the compression in a cross-frame member (force toward the connection) and the positive sign is used for the tension in a cross-frame member (force away from the connection). Therefore, if fit-up forces are calculated in the global direction, the signs in Eq. (4.1) and Eq. (4.2) need to be adjusted for calculation of fit-up forces at connection points to Girder 1 shown in Figure 4.4. 


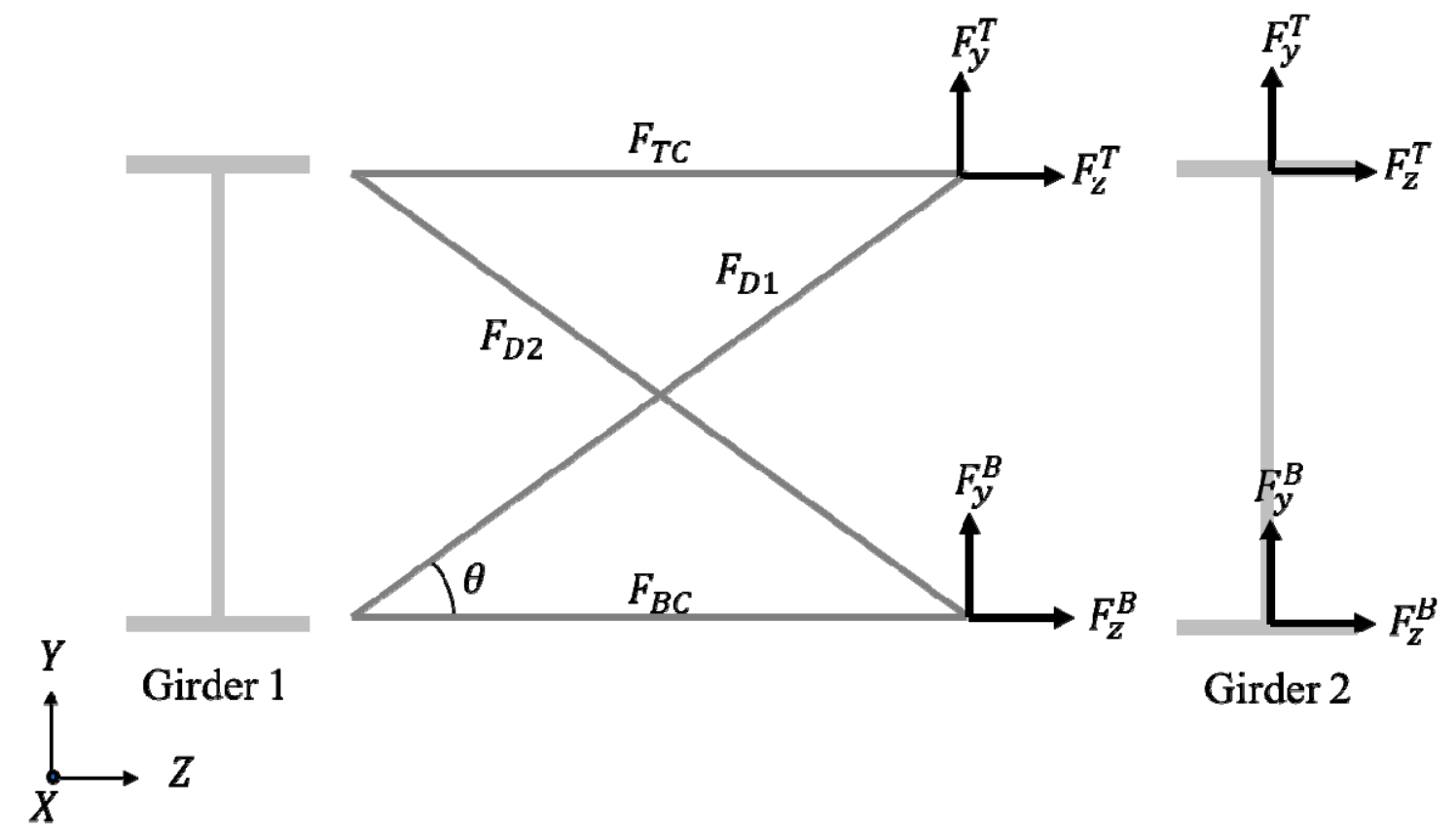

Figure 4.4: Fit-up forces by resolving cross-frame forces at connection points

\subsubsection{D Erection Simulation Method}

The 3D erection simulation attempts to mimic the erection of steel framing in the practice. In this simulation, cross-frames are erected one by one following a particular erection sequence similar to the erection of cross-frames of a real bridge. In practice, the girders are twisted or displaced using a come-along to fit the cross-frame between its connections to girder. Therefore, it is important to know the magnitude of twist or displacement of top and bottom of girders in order for attaching a cross-frame.

Erection simulation has two parts; part A and part B. In part A, the displacements of girders required to fit a cross-frame between its connections to girders are estimated. In part B, these displacements are applied to girders to move them in a position where the cross-frame fit between its connections to girders. Once the girder move into the position, 
cross-frame elements are created between their connections to girders to simulate erection of a cross-frame. A step by step procedure for both parts of the erection simulation is described below.

Part A of the erection simulation consists of two steps for each cross-frame after completing the FEM model of girders being cambered using line girder analysis/isolated girder analysis.

Step 1. In step 1, initial strains are calculated based on the lengths of cross-frame members detailed with the Final Fit detailing method and the distance between the cross-frame and its connections to girders. Each cross-frame member is assigned a particular value of initial strain as shown in Figure 4.5(step 1 of part A). After assigning initial strains to the cross-frame member, the static analysis is run to get the deflected shape of the girders after attaching the cross-frame.

Step 2. From the deflected shape obtained in step 1, the displacements in both vertical and horizontal direction at the top and the bottom of each girder are obtained at the location of the cross-frame erection. The geometry of the structure is updated to be used for calculating initial strain of next cross frame.

Step 1 and Step 2 are repeated for every single cross-frame in the bridge and the displacements are stored. These displacements are used in part B of the erection simulation described as follows.

Part B of the erection simulation consist of three steps for each cross-frame after completing the FEM model of girders being cambered using the line girder analysis or the isolated girder analysis.

Step 1. In step 1, displacements of the connection points calculated from part A of the erection simulation for the cross-frame to be erected are applied on girders as 
shown in Figure 4.5 (step 1 part B). The static analysis is run to get the deformed shape.

Step 2. Once the static analysis is complete, reactions develop at the displacement application points. These reactions are the fit-up forces as shown in Figure 4.5(step 2 part B).

Step 3. Geometry of the structure is updated to the new position to be used for application of displacements and erection of next cross-frame. Cross-frame elements are created at the location of applied displacement as shown in Figure 4.5 (step 3 part B) to simulate the erection of cross-frame.

These steps are repeated for every single cross-frame in the bridge and fit-up forces are obtained for the erection of each cross-frame. It is important to follow same sequence of erection in both Part A and Part B of erection simulation in order to be consistent in using updated bridge geometry. Different erection sequences can be used to erect the cross-frames in the erection simulation. A detailed discussion on different erection sequences is provided in section 4.5.1. 
Bay1 Cross Frame 2

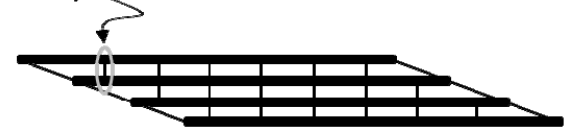

Part A: Estimation of displacements using initial strain (INSTRN) approach
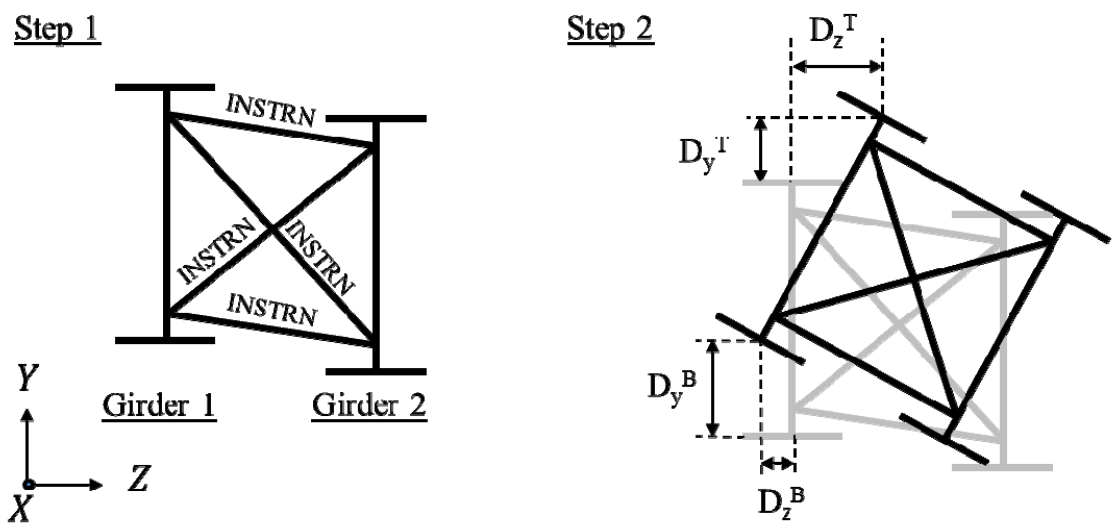

Part B: Application of displacements and calculation of fit-up forces

Step 1

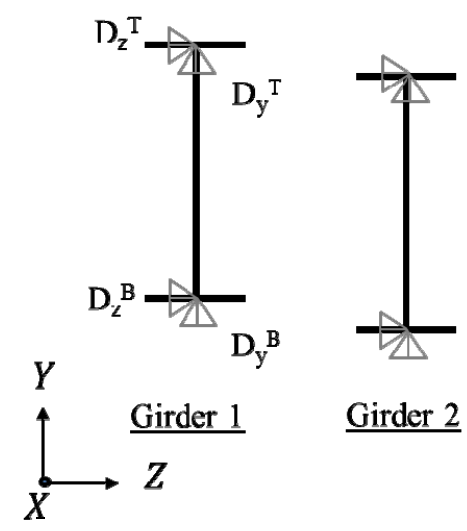

$\underline{\text { Step } 2}$

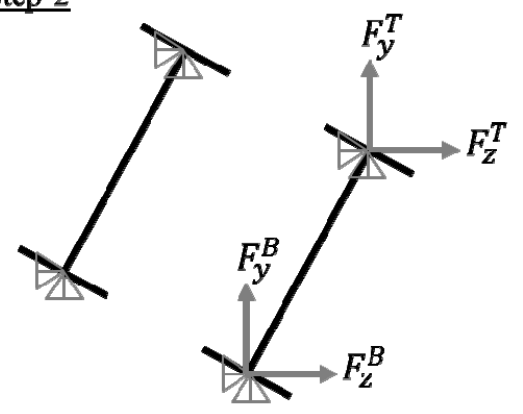

$\underline{\text { Step } 3}$

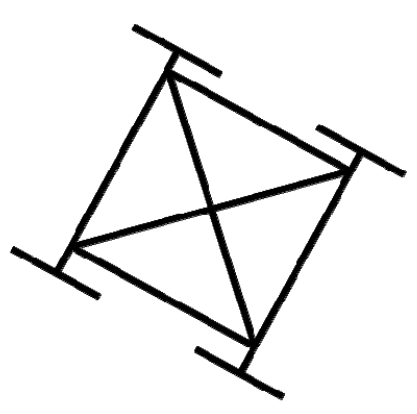

Figure 4.5: Steps followed to calculate fit-up forces in erection simulation.

Bridge girders are generally elastic during the erection of the steel frame of a bridge. The final deflected geometry of elastic girders is not affected by attaching crossframes (CFs) one by one or attaching all cross-frames at once. Therefore, the geometry of girders at the end of the erection simulation (attaching CFs one by one) should be same as the geometry of girders after attaching all cross-frames all at once. Comparison of the geometry of girders (girder layovers and girder elevations) at the end of erection 
simulation (attaching CFs one by one) to geometry of girders after attaching all CFs all at once is done in Figure 4.6 for Girder 4 of Bridge C. As indicated by the Figure 4.6 either attaching the cross-frames all at once or attaching them one by one result in identical final geometry (girder layovers and girder elevations) of Girder 4 of Bridge C. Results shown in Figure 4.6 provides evidence that the erection simulation is working properly. Similar results were obtained for other girders of the Bridge $\mathrm{C}$ as well as Bridge $\mathrm{A}$ and Bridge B.
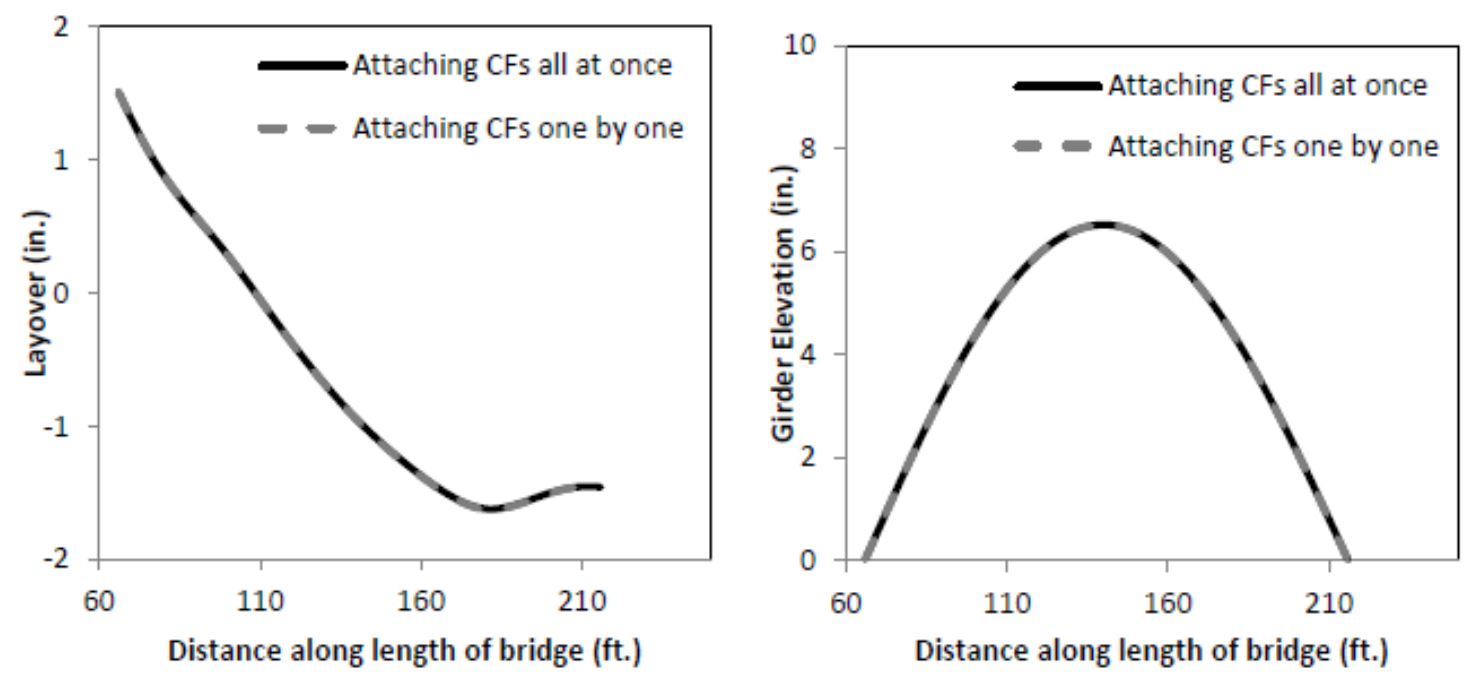

Figure 4.6: Geometry of Girder 4 of Bridge $C$ after completion of erection.

It is important to note that the erection simulation described here has one significant difference to the state of bridge of framing during the erection. In the erection simulation, the geometry of the structure is updated after the erection of each cross-frame that results in the loss of stress developed in the structure due to the erection of previous cross-frame. Therefore, each stage in erection simulation analyzes an unstressed structure with zero forces in the cross-frames already erected. This is different from the state of a 
real bridge during the erection in which the structure is stressed and the erected crossframes have forces in them. However, this difference in the state of the structure in the erection simulation might not effect estimation of fit-up forces significantly. This is because, the steel bridges are not considered as the stress stiffening structures and therefore, absence or presence of a force or a stress in different components of the bridge does not affect the stiffness of the bridge significantly. The change in stiffness of bridge frame during the erection is due to addition of cross-frames. The other factor that significantly affects fit-up forces is the change in geometry of the bridge and accompanied change in the distance between the cross-frames and their connections to the girders. These factors are modeled correctly in erection simulation and match with the real practice of erecting the bridge's steel frame.

\subsection{Discussion and Comparison of Fit-Up Forces}

As discussed earlier, erection of a cross-frame require both lateral and vertical forces at the top and the bottom (FzT, FzB, FyT, FyB) to move the girders into a position where connections can be made between the cross-frame and girders. Comparison of these fit-up forces obtained from the cross-frame force method and the erection simulation method is shown in Figure 4.7 and Figure 4.8 for Girder 3 of Bridge $C$ for erecting cross-frames in Bay 3. The results of the erection simulation method discussed in this section are obtained following Erection Sequence 1. Following observation can be made from by inspecting the data presented in Figure 4.7 and Figure 4.8.

- Lateral fit-up forces at the top and the bottom are in opposite direction indicating that the girder is required to be twisted to make the connections between a crossframe and girders. 
- Vertical fit-up forces for the top and the bottom are generally in the same direction indicating that the girder needs to be moved up or down to make connections between a cross-frame and girders.

- Both lateral and vertical fit-up forces are relatively high for the first intermediate cross-frame because; a) the distance between the cross-frame and connection point is highest for this cross-frame and b) bridge's frame have large stiffness near the obtuse corners and require more force for fitting the cross-frame.

- The highest fit-up force calculated from the cross-frame force method is in good agreement with the highest fit-up force calculated from the 3D erection simulation method.

(Note: Figure 4.7 and Figure 4.8 use CF Force Method to connote cross-frame force method, and Erect. Simul. Method to connote Erection Simulation Method)
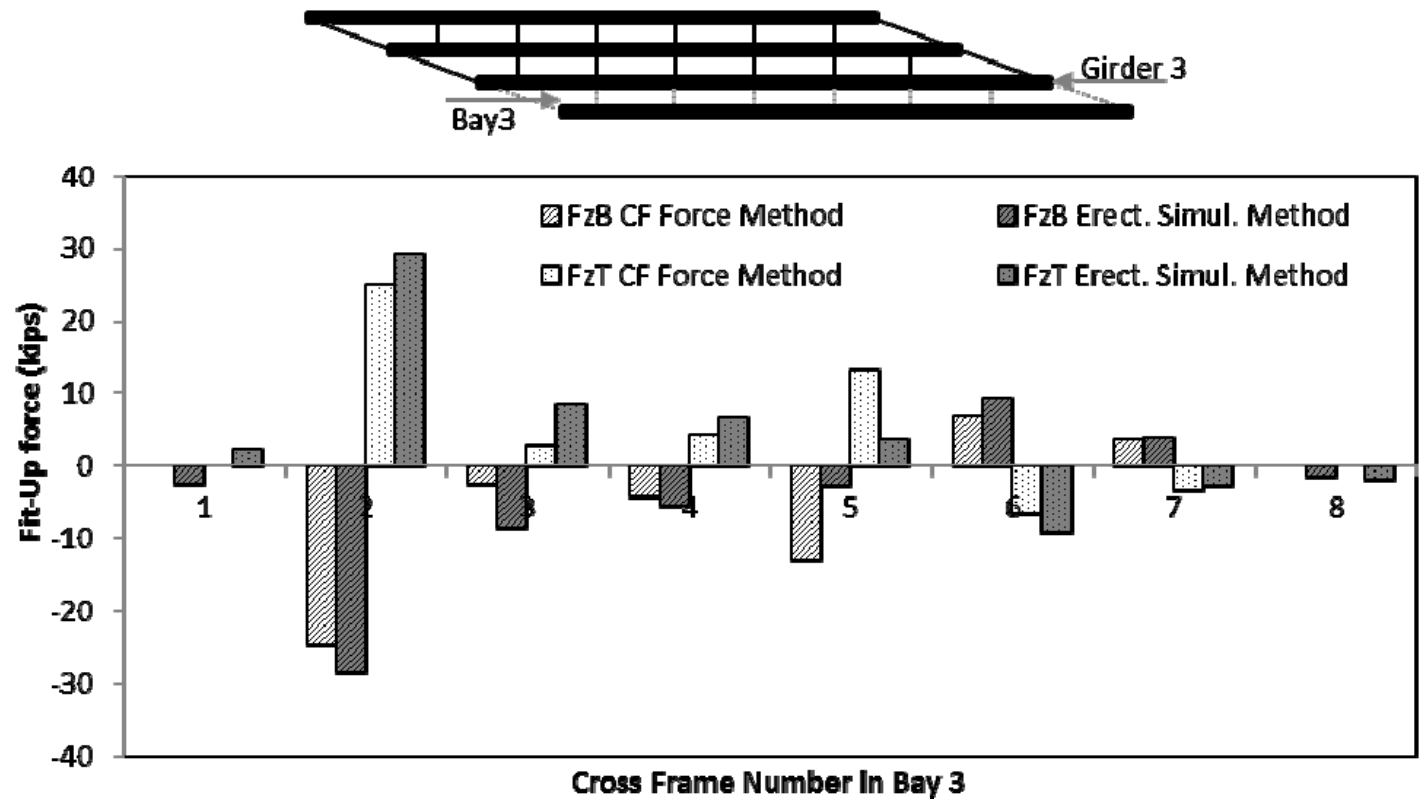

Figure 4.7: Lateral Fit-up forces applied on Girder 3 of Bridge $C$ for erecting crossframes in Bay 3 




Figure 4.8: Vertical Fit-up forces applied on Girder 3 of Bridge $C$ for erecting crossframes in Bay 3

Generally, the erector is interested in knowing the maximum level of fit-up force required to fit the cross-frames between its connections to girders during the erection of a steel bridge. Therefore, absolute maximum fit-up force in both vertical and lateral direction is obtained from different methods of analysis for Bridge A, Bridge B and Bridge $\mathrm{C}$ as shown in Table 4.1. It can be noticed that fit-up forces calculated from crossframe forces are in reasonable agreement with the fit-up forces obtained from the erection simulation except for Bridge A. For Bridge A, cross-frame forces method overestimates the fit-up forces because the cross-frame forces are overestimated by the improved 2D grid analysis for this bridge. This overestimation of cross-frame forces by the improved 2D grid analysis for Bridge $\mathrm{A}$ is discussed in detail in section 3.2.5. In summary, crossframes forces obtained from the improved 2D grid analysis can be used to estimate fit-up forces required for the Final Fit detailing method at the erection stage. 
Table 4.1: Absolute Maximum Fit-Up force from different methods

Absolute Maximum Fit-Up force (kips) Lateral

\begin{tabular}{cccc}
\hline & Lateral & & Vertical \\
\hline Cross-Frame & Erection & Cross-Frame & Erection \\
Forces Method & Simulation & Forces Method & Simulation \\
\hline 230 & 180 & 104 & 131 \\
41 & 38 & 28 & 30 \\
25 & 29 & 7 & 10 \\
\hline
\end{tabular}

It is important to mention here that cross-frame forces are function of cross-frame stiffness or cross section area of cross-frame members as shown in section 5.4.1. Whereas, the fit-up forces may or may not be a function of cross-frame stiffness depending on whether or not, the girder to be moved is attached with cross-frames. Since, the maximum fit-up force is generally required to move the girder that is attached with the cross-frames, the effect of cross-frame stiffness is portrayed in the fit-up forces similar to the cross-frame forces.

In order to further investigate the good agreement between the fit-up forces calculated from the erection simulation and cross-frame forces, the erection simulation is used to monitor the change in cross-frame forces during the erection of a skewed bridge. The force in the top chord and the bottom chord of $1^{\text {st }}$ cross-frame in bay 1 of Bridge $\mathrm{C}$ during different stages of the erection is shown in Figure 4.9. Results shown in Figure 4.9 are obtained from the erection simulation using Erection Sequence 1 (erecting crossframe starting from left end of a bay and moving toward right end of the bay). This way the first cross-frame in bay 1 is erected at the start of the erection and last cross-frame in bay 3 is erected at the end of the erection. The force in the top chord and the bottom chord keep on changing with the erection of other cross-frames. The cross-frame force in the first cross-frame is affected significantly only when a cross-frame in line with this 
first cross-frame is erected. The cross-frames in the last bay are erected at the very end of the erection and therefore, the forces in these cross-frames do not change significantly. Fortunately, the fit-up forces are highest for the erection of cross-frames in the last bay. Therefore, the magnitude of the maximum fit-up force estimated from the cross-frame forces is in good agreement with the magnitude of the maximum fit-up force estimated from the erection simulation.

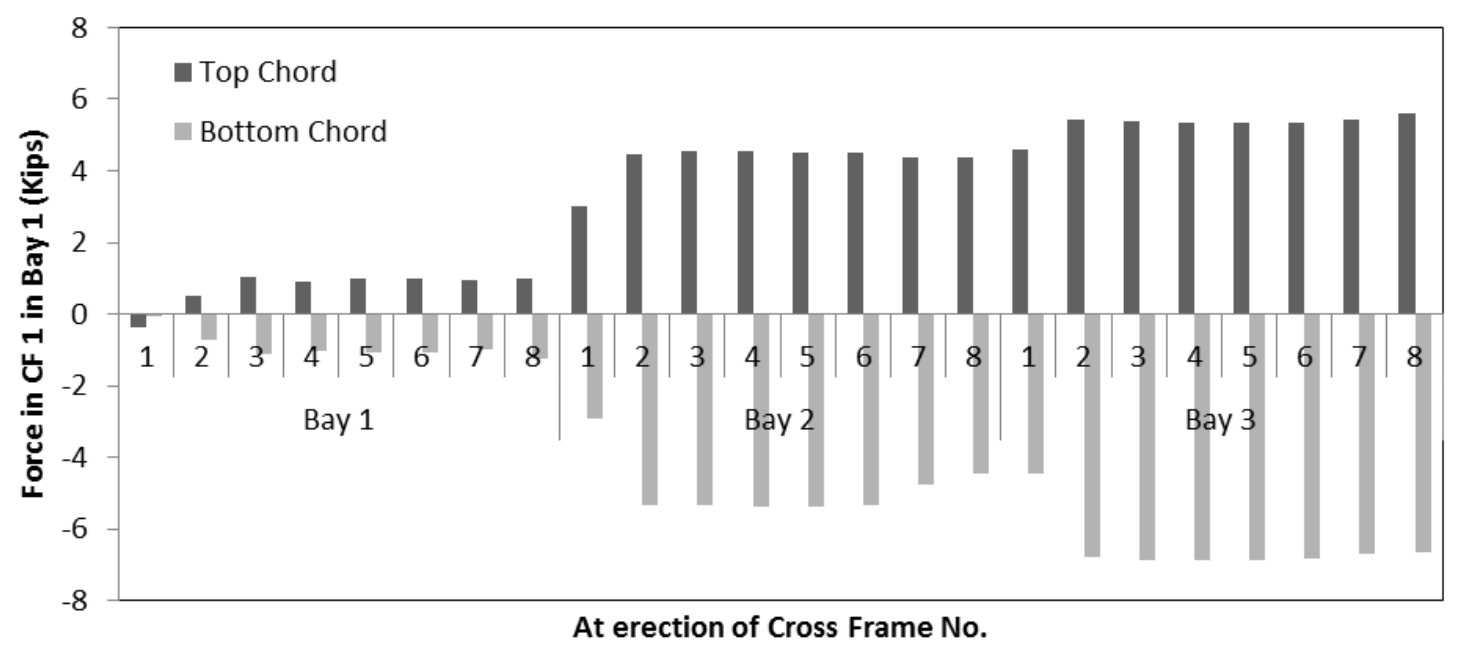

Figure 4.9: Cross-frame forces in $1^{\text {st }}$ cross-frame of Bay 1 of Bridge $C$ during the erection

\subsection{Effect of Different Practices on Fit-Up Forces}

Different construction and detailing practices also affect the fit-up forces. These construction and detailing practices include the followings:

- Different erection sequences

- Distance of the first intermediate cross-frame from the support 
These effects are evaluated by carrying out the erections simulation and the $3 \mathrm{D}$ FEM analysis. Detailed discussion on the effect of each construction and detailing practice on the fit-up forces is provided in the following sections.

\subsubsection{Different Erection Sequences}

Three different erection sequences that can be followed for attaching the crossframe to their connections to girders are shown in Figure 4.10. In erection sequence 1, cross-frames are attached starting from one end of a bay and moving toward the other end of the bay. In erection sequence 2, the cross-frames are attached starting from the two ends of a bay and moving toward the middle of the bay. In erection sequence 3 , crossframes are attached starting from the middle of a bay and moving outward toward the ends of the bay.

\section{Erection Sequence 1}

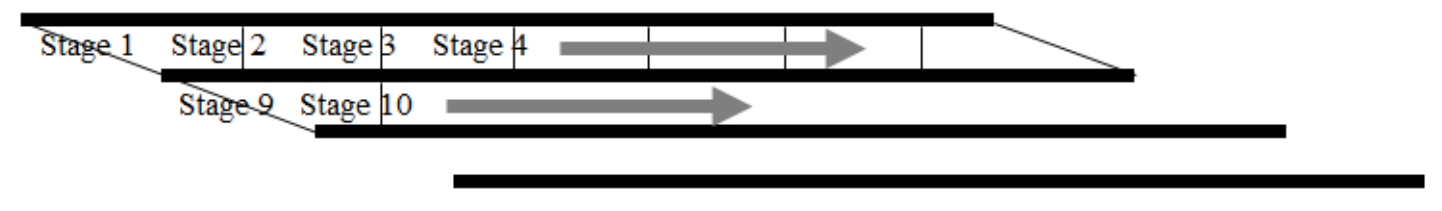

Erection Sequence 2

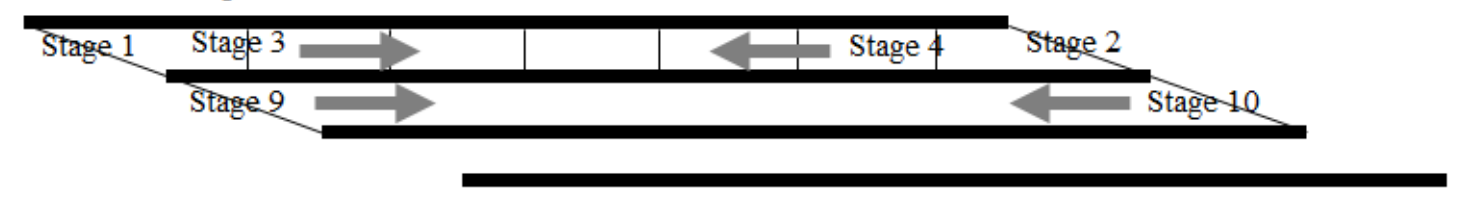

Erection Sequence 3

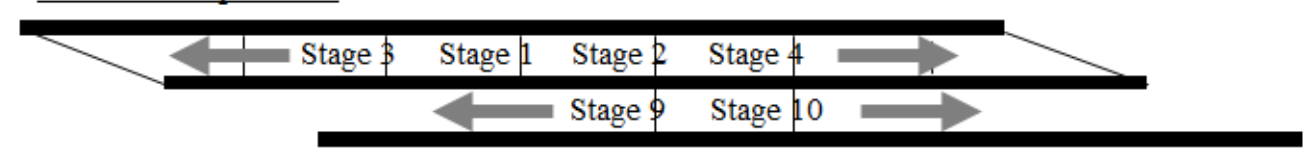

Figure 4.10: Erection sequences for attaching cross-frames to girders 
Fit-up forces are evaluated from 3D erection simulation following the three erection sequences to find out the erection sequence that requires minimum fit-up force. These fit-up forces for Girder 8 of Bridge A for erection of cross-frame in Bay 8 are compared in Figure 4.11. Following observations can be made by inspecting the data presented in Figure 4.11:

- Large fit-up forces are required to erect the cross the cross-frames near the obtuse corner of the bridge.

- Less maximum fit-up force is required if erection sequence 3 is followed compared to the maximum fit-up force required by following erections sequences 1 and 2.

- Fit-up forces are more evenly distributed for erection sequence 3 compared to the distribution of fit-up forces in erection sequences 1 and 2.

These observations can be partly explained by the following discussion. Fit-up forces for the erection of a particular cross-frame depend on the distance between the cross-frame and its connection points, and the vertical and the torsional stiffness of girders in a bay. As shown in Figure 2.3, the distance between the cross-frames and their connection points is small for the cross-frames in the middle of a bay compared to the cross-frames at the ends of the bay. This distance between cross-frame and their connection points change during the erection of cross-frames because erection of each cross-frame deflect the girders into a new position. Generally, this distance between the cross-frames and their connection points decreases with the increase in the number of cross-frames attached to the girder during the erection. The torsional and the vertical stiffness of the girders do not change significantly during the erection of cross-frames in a bay. 
In erection sequence 3, the cross-frame that has less displacement between their connections to girders are attached first and thereby gradually deflecting the girders and decreasing the distance between the connections for the end cross-frames. Therefore, the maximum fit-up force is less in erection sequence 3 compared to the maximum fit-up force in erection sequences 1 and 2. In erection sequences 1 and 2 the cross-frame near the end of the bay are erected first and girders need to be displaced through a large distance to make the connections thereby requiring relatively larger forces.

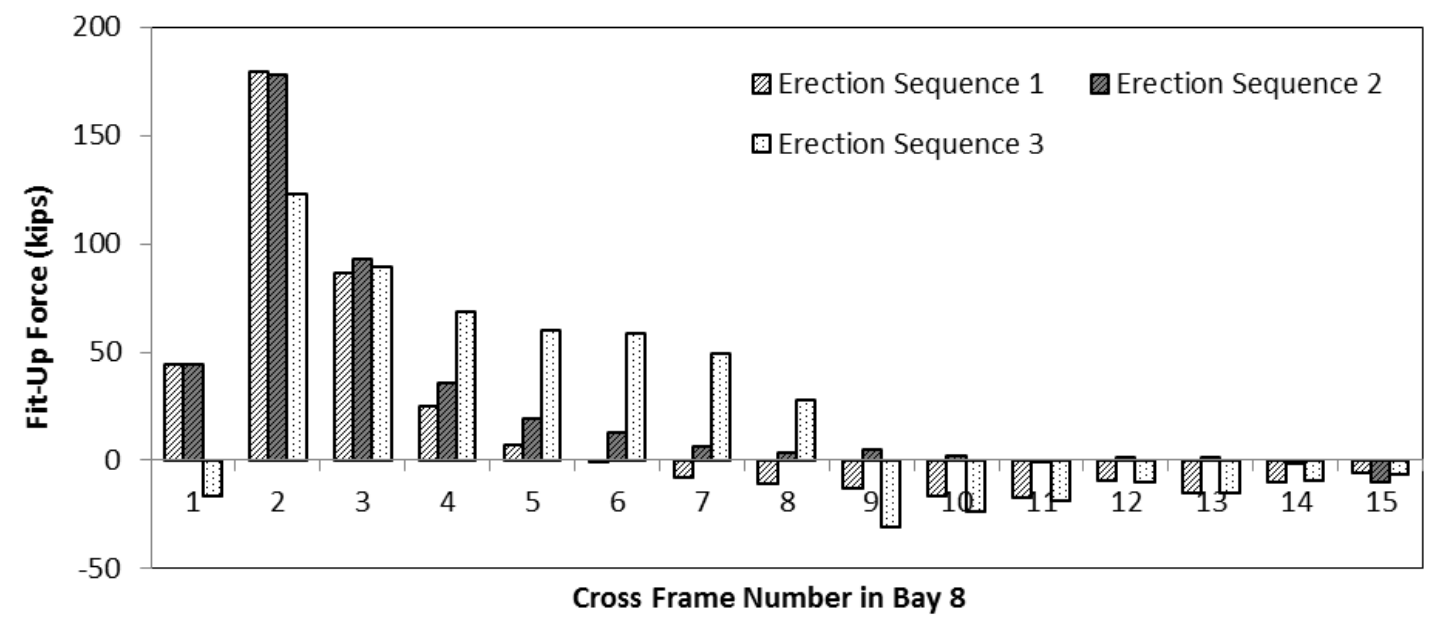

Figure 4.11: Fit-up force at the top of Girder 8 of Bridge A for erecting the crossframes in Bay 8

Also note that the stiffness of the bridge's frame is large near the obtuse corners compared to the stiffness at the acute corners and therefore, the cross-frame near the obtuse end of a bay require more fit-up force compared to fit-up force required to erect the cross-frame near the acute end as shown in Figure 4.11. 
In summary less maximum fit-up force is required if cross-frames are attached using erection sequence 3 that is attaching the cross-frames in the middle of a bay first and proceeding toward the end of the bay.

In order to verify the conclusion drawn from Figure 4.11 the maximum fit-up force is calculated for Bridge A, Bridge B and Bridge $\mathrm{C}$ following the three different erection sequences and is shown in Table 4.2. As shown in Table 4.2 the maximum fitup force is less following erection sequence 3 for the three bridges in both vertical and lateral direction.

Table 4.2: Absolute Maximum Fit-Up force from different erection sequences Absolute Maximum Fit-Up force (kips)

\begin{tabular}{|c|c|c|c|c|c|}
\hline \multicolumn{3}{|c|}{ Lateral } & \multicolumn{3}{|c|}{ Vertical } \\
\hline $\begin{array}{c}\text { Erection } \\
\text { Sequence } 1\end{array}$ & $\begin{array}{c}\text { Erection } \\
\text { Sequence } 2\end{array}$ & $\begin{array}{c}\text { Erection } \\
\text { Sequence } 3\end{array}$ & $\begin{array}{c}\text { Erection } \\
\text { Sequence } \\
1\end{array}$ & $\begin{array}{c}\text { Erection } \\
\text { Sequence } \\
2\end{array}$ & $\begin{array}{c}\text { Erection } \\
\text { Sequence } \\
3\end{array}$ \\
\hline 180 & 178 & 123 & 131 & 131 & 89 \\
\hline 38 & 40 & 32 & 31 & 32 & 25 \\
\hline 29 & 30 & 20 & 10 & 11 & 8 \\
\hline
\end{tabular}

\subsubsection{Distance of the First Intermediate Cross-frame from Support}

The distance of the first intermediate cross-frame from the support also affects the fit-up forces. In section 4.4, it has been shown that the fit-up forces can be calculated from the cross-frame forces. Effect of the distance of the first intermediate cross-frame from the support on the cross-frame forces is evaluated in a parametric study shown in section 5.4.2. The cross-frame forces decreases with the increase in the distance of the intermediate cross-frame from the support; however, the decrease in the cross-frame forces is not very significant. Therefore, it is expected that maximum fit-up forces 
decrease with the increase in the distance of the first intermediate cross-frame from the support; however, not significantly.

\subsection{Summary}

This chapter discusses the fit-up forces that are required to fit cross-frames, detailed with the Final Fit detailing method, between their connections to the girders during the erection or the steel dead load stage. This chapter introduces the $2 \mathrm{D}$ grid analysis method and the 3D finite element method to calculate the fit-up forces. The main conclusion of this chapter is that cross-frame forces estimated from the improved 2D grid analysis can be used to calculate the maximum fit-up force. The erection simulation has shown that erecting the cross-frame staring from the middle of a bay and moving toward end of the bay (erection sequence 3) require less maximum fit-up force compared to other erection sequences.

Chapter 2, 3 and 4 discuss different issues related to framing of skewed bridges and provide elements to develop a complete and simplified design approach that can be used in practice. The following chapter develops a simplified and coherent design approach by evaluating the importance of different framing layouts, structural responses

associated with detailing methods, and recommending methods of calculating cambers in skewed bridges. 


\section{DESIGN PROVISIONS}

Different structural responses related to different detailing methods have been compared for different skewed bridges in previous studies [1] [2] [3][6] [7] [8] and in the previous chapters of this dissertation. These studies provide the foundation and elements that are needed to develop a comprehensive and coherent design, fabrication, and construction approach for straight and skewed steel bridges.

The main objective of this chapter is to simplify the design and construction of steel bridges with skewed supports by introducing a complete and coherent design approach. This objective is achieved by the following:

1) Recommending the method of analysis for the calculation of the camber for different detailing methods,

2) Identifying important structural responses and recommendation of methods of analyses for obtaining these responses,

3) Evaluating the effect of different framing options on different structural responses of the bridges, and

4) Developing a flow chart that can help designers to choose the appropriate detailing method for detailing the cross-frames.

\subsection{Recommendation on Calculation of Cambers}

The cambers need to be estimated correctly in skewed bridges, because there is no conservative side in the estimation of cambers. Incorrect estimation of cambers either above or below the correct values results in potential lack-of-fit effects and change in bridge cross slopes. These problems can potentially lead to expensive retrofits, delays in construction, claims, and litigations. 
Different methods of analysis can result in different cambers in skewed bridges. For example, cambers calculated from the line girder analysis are different from the cambers calculated from the 2D grid analysis. This is because in skewed bridges, girder's vertical deflections at different loading stages are affected by the lack-of-fit depending on the detailing method. Lack-of-fit effects include a component of the vertical deflection due to the lack-of-fit of cross-frames between their connections to girders as discussed in detail in section 2.5.2. This component of the vertical deflection due to the lack-of-fit might be permanent or temporary depending on the detailing method used. Therefore, depending on the detailing method used, cambers can be correctly estimated by either the line girder analysis or the grid analysis or a combination of the line girder and the grid analysis.

For the Erected Fit detailing method, the SDL cambers should be estimated by the line girder analysis because there is no component of the vertical deflection due to lackof-fit in girders at the SDL stage. The concrete dead load (CDL) cambers should be calculated by the 2D grid analysis or the 3D FEM analysis modeling all the girders and cross-frames connected together. This is because the CDL move the girders into a position where cross-frame don't fit between their connections to girders and result in lack-of-fit. This lack-of-fit and accompanied component of the vertical deflection is permanent and therefore should be included into the camber calculation.

For the Final Fit detailing method both SDL and CDL cambers need to be calculated by the line girder analysis. This is because in the Final Fit detailing method cross-frames are detailed to fit between the girders at the TDL stage. Therefore, there is no lack-of-fit and associated component of the vertical deflection in the girders at the 
TDL stage. It should be noted that in the Final Fit detailing method lack-of-fit and component of the vertical deflection appears at the SDL stage. However, this lack-of-fit and accompanied component of the vertical deflection is temporary and goes away once the CDL is applied. Further, if cambers from the grid analysis are used for the Final Fit detailing method the cross-frame do not fit between the girders at the TDL stage and therefore result in lack-of-fit at the TDL stage as shown in previous studies [7] [8] [9].

Table 5.1: Method of calculation of camber for different detailing methods

Detailing Method

\section{Method of calculation of camber for}

\begin{tabular}{llc}
\cline { 2 - 3 } & Steel Dead Load & Concrete Dead Load \\
\hline Erected Fit & Line Girder Analysis & 2D Grid Analysis \\
Final Fit & Line Girder Analysis & Line Girder Analysis \\
\hline
\end{tabular}

\subsubsection{Verification of Recommendation Using Numerical Models}

In order to verify the recommendation on camber calculation, the cambers calculated from the recommended analyses are incorporated in the 3D FEM analysis followed by application of dead load in the construction sequence; placing girders on supports, attaching the cross-frame, and applying the dead loads. For the Final Fit detailing method, there is a lack-of-fit between cross-frames and their connections to girders at the SDL simulated by initial strains calculated from the camber diagram see 3.4.2 for details, whereas for the Erected Fit detailing method cross-frames are attached to the girders without initial strains at the SDL stage. 
Three simply supported I-girder bridges, Bridge A, Bridge B and Bridge C, having different levels of skew are selected for consideration in this study. All three bridges have their girders and cross-frames designed with Grade 50 steel having a modulus of elasticity of 29,000 ksi. Detailed geometry, framing plan and sizes of different components for these bridges are shown in Appendix A.

Following discussion explains the numerical analysis results of the bridges described above and provides the evidence that recommendations regarding camber calculation, shown in Table 5.1, are correct. Figure 5.1 to Figure 5.6 show the vertical deflections calculated from the recommended method of analysis and the verification of camber recommendation by the 3D FEM analysis for Bridge A, Bridge B and Bridge C. For example, Figure 5.1(a) shows the TDL vertical deflection of the girder 1 of Bridge A calculated by a combination of the line girder analysis (1D LGA) and the improved 2D grid analysis (2D GA) as per the recommendation for the Erected Fit detailing method. Figure 5.1(b) shows the camber in Girder 1 in the 3D FEM model of Bridge A at different loading stages. At the no load (NL) Stage the camber in the girder is equal to the TDL vertical deflection calculated as per recommendation. The line corresponding to the SDL stage in Figure 5.1(b) shows the camber in Girder 1 after application of the steel dead load. Since, for the Erected Fit detailing method, cross-frames are detailed to fit between their connections to girders at the SDL stage, attaching cross-frames to girders at the SDL stage does not cause a component of the vertical deflection due to lack-of-fit. Therefore, cambers at the SDL stage for the Erected Fit detailing method are same before and after attaching the cross-frame. The line corresponding to the TDL stage in Figure 5.1(b) shows the remaining cambers in Girder 1 of the bridge's 3D FEM model after 
application of the TDL. If the camber recommendations are correct the girder should be flat with zero camber at the TDL stage.

Similarly Figure 5.2 (a) shows the TDL vertical deflection of Girder 1 of the bridge calculated by line girder analysis (1D LGA) only, as per recommendation for the Final Fit detailing method. Figure 5.2(b) shows the camber in Girder 1 of the bridge's 3D FEM model at different loading stages. At the no load (NL) stage the camber in the girder is equal to the TDL vertical deflection calculated as per recommendation. There are two lines for the SDL stage in Figure 5.2(b). The dashed line shows the camber in girder 1 after application of the steel dead load (SDL) before attaching the cross-frames. The dotted line shows the camber in Girder 1 after application of the steel dead load (SDL) after attaching the cross-frames. This is because the cross-frames, detailed with the Final Fit detailing method, do not fit between the girders at the SDL stage. Therefore, attaching the cross-frames, detailed with Final Fit detailing method, at the SDL stage is accompanied by a component of the vertical deflection due lack-of-fit of cross-frames between their connections to girders. Due to this component of the vertical deflection, cambers are different before and after attaching the cross-frame for the Final Fit detailing method as shown in Figure 5.2(b). The line corresponding to the TDL stage in Figure 5.2(b) shows the remaining cambers in Girder 1 of the bridge's 3D FEM model after application of the TDL. Again, if the camber recommendations are correct the girder should be flat with zero camber at the TDL stage.

It is worth mentioning that in the case of Bridge A and Bridge B the vertical deflection due to the weight of steel girders is close to the vertical deflection due to the concrete dead weight, because of the girder sizes and girder spacing. In most usual cases, 
one would expect to have a SDL vertical deflection to be about $30 \%$ of the total dead load vertical deflection. This is the case for Bridge $\mathrm{C}$.

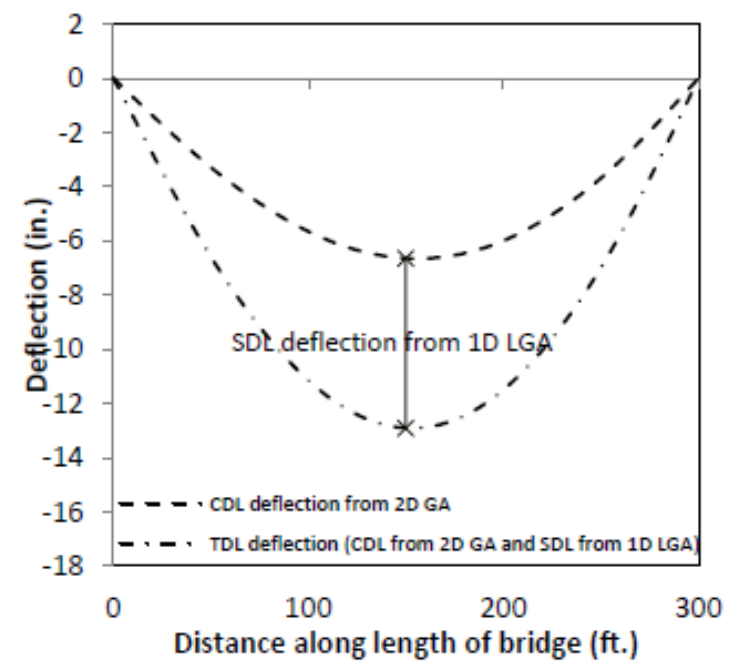

a) Calculation of camber from 1D LGA and 2D GA as per recommendation

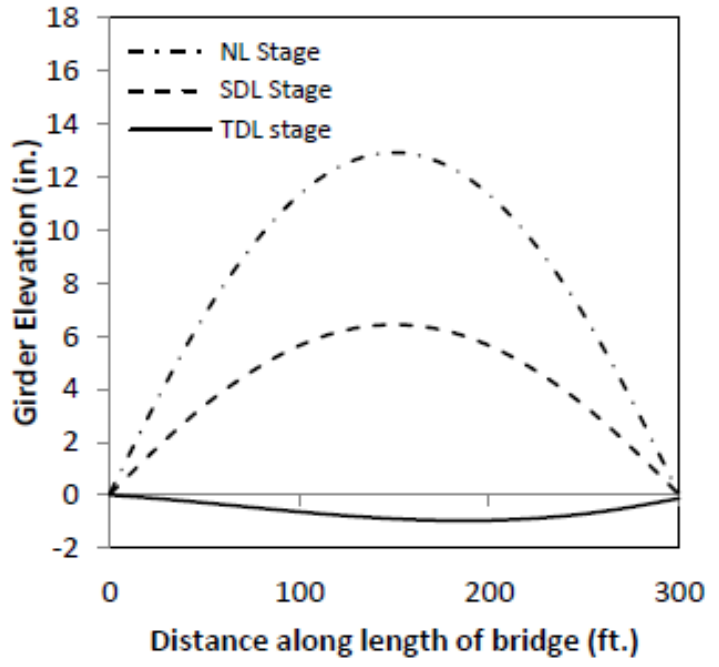

b) Verification of recommendation by $3 \mathrm{D}$ FEM analysis

Figure 5.1: Verification of camber recommendation for the Erected Fit detailing method-Bridge A Girder 1

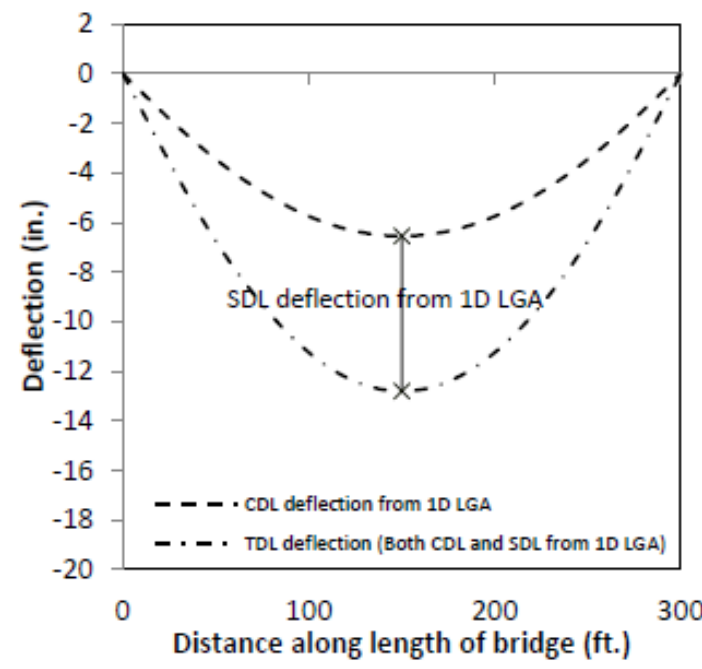

a) Calculation of camber from 1D LGA as per recommendation

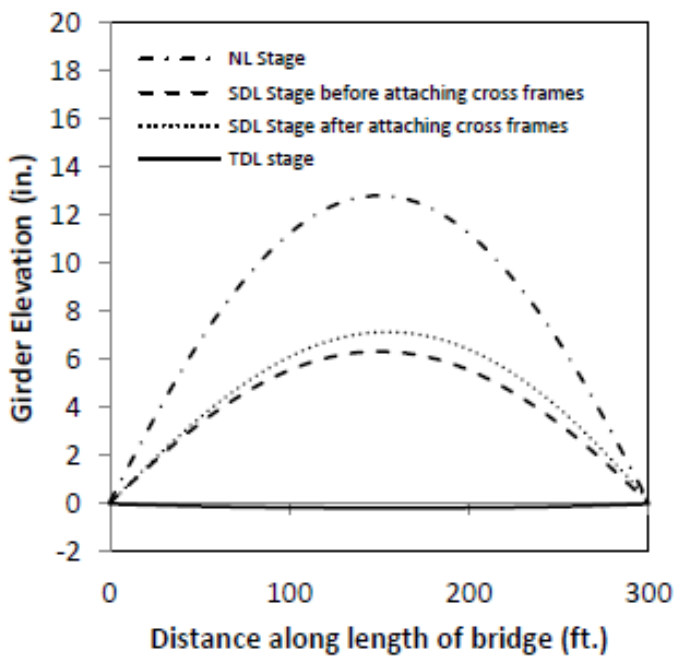

b) Verification of recommendation by 3D FEM analysis

Figure 5.2: Verification of camber recommendation for the Final Fit detailing method-Bridge A Girder 1 


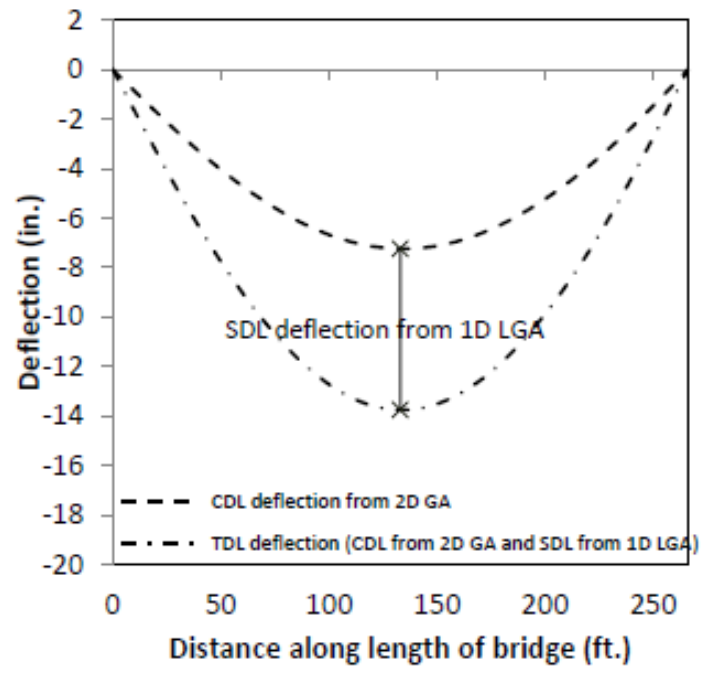

a) Calculation of camber from 1D LGA and 2D

GA as per recommendation

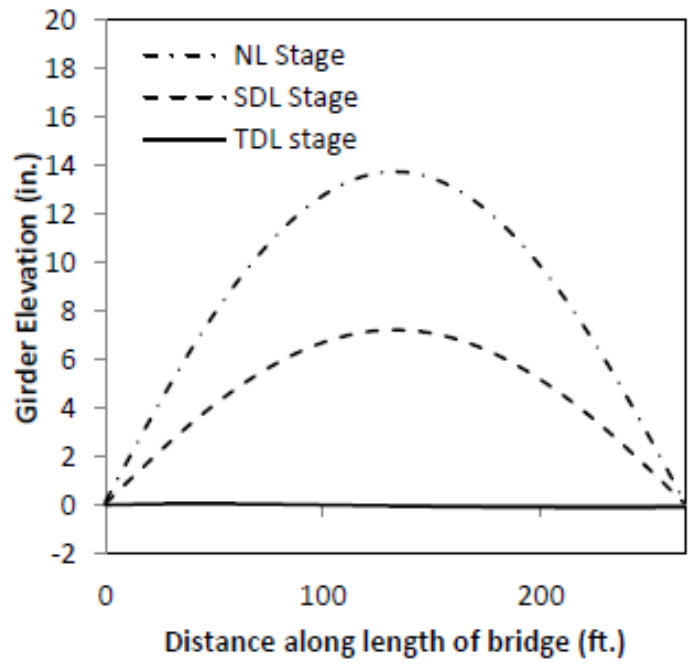

b) Verification of recommendation by 3D FEM analysis

Figure 5.3: Verification of camber recommendation for the Erected Fit detailing method-Bridge B Girder 1

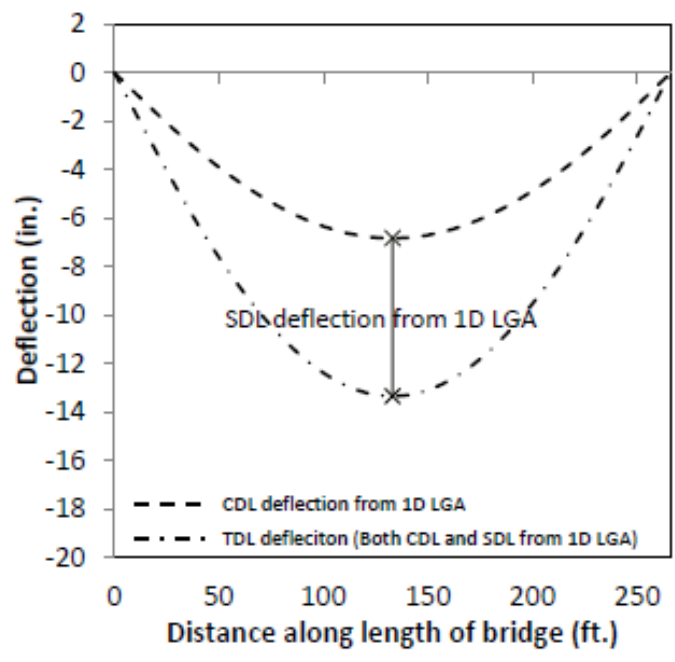

a) Calculation of camber from 1D LGA as per recommendation

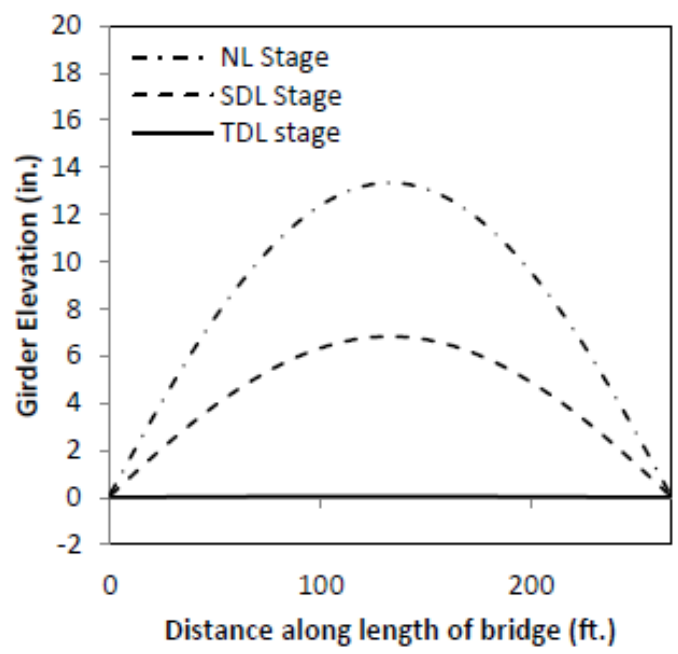

b) Verification of recommendation by 3D FEM analysis

Figure 5.4: Verification of camber recommendation for the Final Fit detailing method-Bridge B Girder 1 


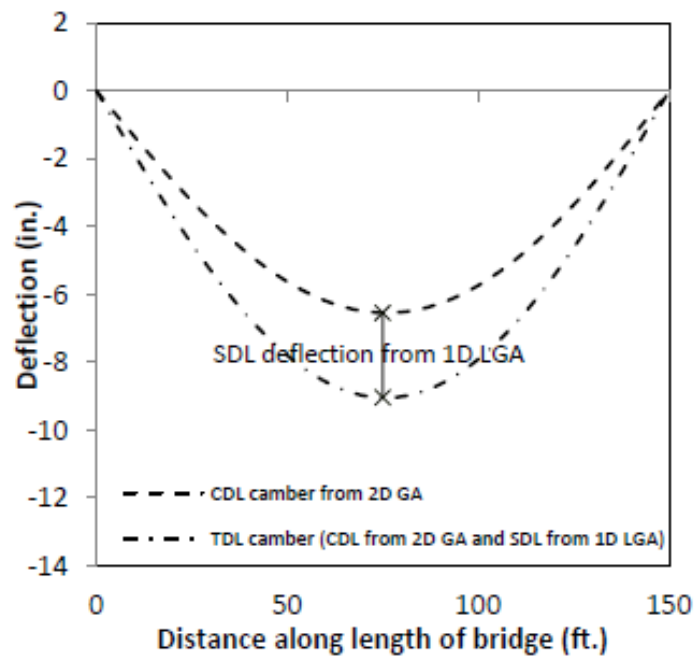

a) Calculation of camber from 1D LGA and 2D GA as per recommendation

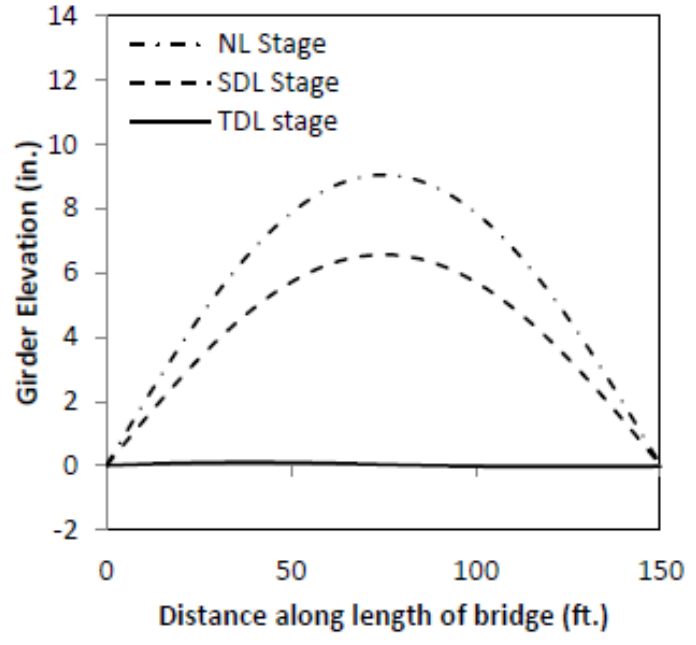

b) Verification of recommendation by 3D FEM analysis

Figure 5.5: Verification of camber recommendation for the Erected Fit detailing method-Bridge $C$ Girder 1

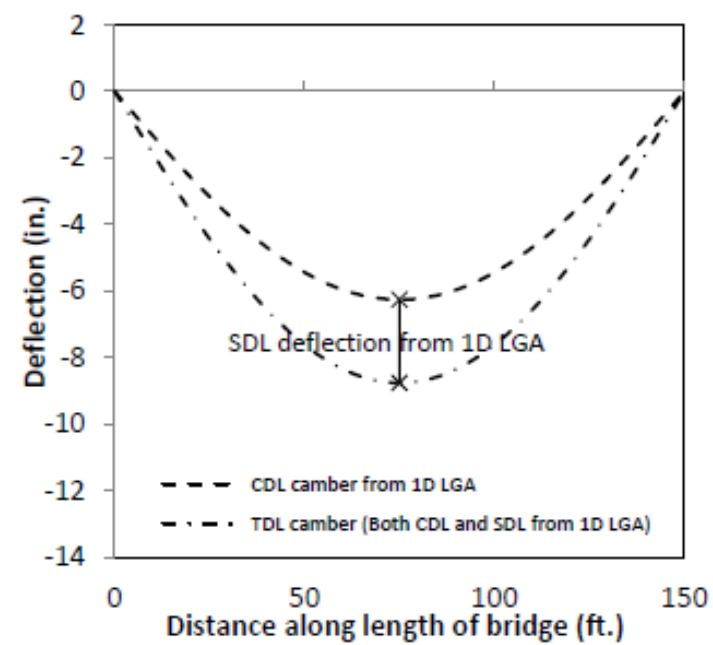

a) Calculation of camber from 1D LGA as per recommendation

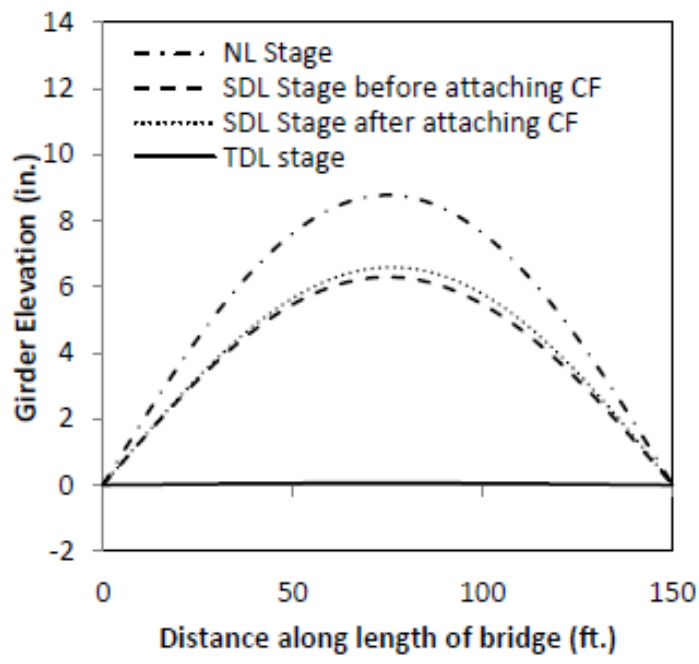

b) Verification of recommendation by 3D FEM analysis

Figure 5.6: Verification of camber recommendation for the Final Fit detailing method-Bridge C Girder 1 


\subsubsection{Summary and Discussion on Numerical Analysis}

Summary of results obtained from the numerical analysis done in section 5.1.1 is shown in Table 5.2for the three bridges. Table 5.2 shows the maximum difference from flat or zero line at the TDL stage considering all the girders in the bridge for both Erected Fit and Final Fit detailing method. It can be observed from Table 5.2 that maximum difference from zero line at the TDL stage is less than $2 \%$ for all the bridges for both Erected Fit and Final Fit detailing method except for the Erected Fit detailing method for Bridge A. It is also worth noting that magnitude of error (difference from the zero line at the TDL stage) is less in the Final Fit detailing method compared to the Erected Fit detailing method. This is because the $2 \mathrm{D}$ grid analysis used in calculation of the concrete dead load vertical deflections in case of the Erected Fit detailing. In the 2D grid analysis the torsional stiffness of the girders is estimated by using an equivalent torsional constant $\left(J_{e q}\right)$. This torsional constant approximates the torsional stiffness of girders and has an effect on the concrete dead load (CDL) vertical deflections of girders. In most of the bridges, $J_{e q}$ does a good job in approximating the torsional stiffness of the girder; however, when bridge has staggered cross-frames with very small staggered distance (Bridge A), the unbraced length $\left(L_{b}\right)$ gets very small and $J_{e q}$ gets very large. High $J_{e q}$ value gives an artificially high torsional stiffness to the girders decreasing their vertical deflections. Therefore, the CDL vertical deflections obtained from the 2D grid analysis are relatively low compared to the CDL vertical deflection obtained from 3D FEM analysis. 
Other sources of errors correspond to differences in boundary conditions (3D FEM Vs. 1D LGA and 2D GA) and the difficultly in building exact cambers in the 3D FEM models.

Table 5.2: Summary of camber analysis

\begin{tabular}{lcccc}
\hline & \multicolumn{3}{c}{ Maximum difference from zero line at the TDL stage } \\
\cline { 2 - 5 } & inches & \%age of TDL camber & inches & \%oage of TDL camber \\
\hline Bridge A & -1.01 & $-9.4 \%$ & -0.21 & $-1.6 \%$ \\
Bridge B & -0.09 & $-0.7 \%$ & 0.06 & $0.5 \%$ \\
Bridge C & 0.14 & $1.8 \%$ & 0.06 & $0.7 \%$ \\
\hline
\end{tabular}

NOTE: A negative difference shows that girders are deflected below the zero line at the TDL stage

\subsection{Structural Responses of the Skewed Bridges Affected By Detailing Methods}

Different structural responses of skewed bridges affected by detailing methods are referred as lack-of-fit effects and are discussed at length in chapter 2. Table 5.3summarizes the lack-of-fit effects associated with Erected Fit and Final Fit detailing methods presented in chapter 2 .

As indicated in Table 5.3, different lack-of-fit effects associated with Erected Fit and Final Fit methods appear at different stages of the construction. Lack-of-fit effects for the Erected Fit detailing method are zero at the SDL stage and are significant at the TDL stage. Converse is true for the Final Fit detailing method. In the discussion to follow, importance of each lack-of-fit effect is discussed to make design recommendations.

Girder layovers are not of any structural consequence, as long as the load levels are less than a small fraction of the critical elastic buckling load at the factored strength load levels, i.e., they do not have any significant impact on the strength of the structural system as discussed in section 2.5.1. 
The component of vertical reaction due to lack-of-fit $\left(\mathrm{R}_{\mathrm{Y} 2}\right)$ can be downward (positive) or upward (negative) depending on the location of the bearing in the bridge support. If the magnitude of the upward $\mathrm{R}_{\mathrm{Y} 2}$ at a particular bearing exceeds the magnitude of the downward dead load vertical reaction at the bearing, uplift is observed at that bearing. This uplift is more common in case of the Final Fit detailing method since, $\mathrm{R}_{\mathrm{Y} 2}$ appear at the SDL loading stage at which the downward dead load vertical reaction is only from the SDL. However, the SDL stage is temporary stage and the $\mathrm{R}_{\mathrm{Y} 2}$ is zero at the TDL stage. For the Erected Fit detailing method, $\mathrm{R}_{\mathrm{Y} 2}$ appear at the TDL stage, however, the negative vertical reaction is not observed, because of the high positive dead load vertical reaction at the TDL stage.

The component of the vertical deflection due to lack-of-fit $\left(\mathrm{D}_{\mathrm{Y} 2}\right)$ for the Final Fit detailing method appears at the temporary SDL stage and is zero at the TDL stage. Therefore, it should not be included in calculation of cambers for the Final Fit detailing method. For the Erected Fit detailing method, $\mathrm{D}_{\mathrm{Y} 2}$ appear at the permanent TDL stage and therefore should be included in the camber. Further, it should be noted that the magnitude of $\mathrm{D}_{\mathrm{Y} 2}$ varies from bridge to bridge. Maximum value of $\mathrm{D}_{\mathrm{Y} 2}$ for Bridge $\mathrm{A}$ is 2.1 inch, for Bridge B is 0.6 inch and for Bridge $C$ is 1.0 inch. These values can be considered as significant given the fact that skewed bridges face difficulty in meeting tolerance limits. Therefore, it is recommended to include the $\mathrm{D}_{\mathrm{Y} 2}$ in to the design of skewed bridge by following the recommendations on camber calculation.

The flange lateral bending stress $\left(f_{l}\right)$ is important for stability of girder flanges. For the Final Fit detailing method, $f_{l}$ appears at the SDL stage due to the lack-of-fit of cross-frames between their connections to girders. At the SDL stage, $f_{l}$ can also appear 
from the wind load regardless of the detailing method. However, at the SDL stage, girder's major axis bending stress is at low level being caused by only the SDL. For the Erected Fit detailing method, $f_{l}$ appears at the TDL stage. The lateral load from knee braces is another source of $f_{l}$ at the TDL stage. At the TDL stage, girder's major axis bending stresses are high due to the presence of both SDL and CDL on the structure. Therefore, $f_{l}$ can be critical for both Erected Fit and Final Fit detailing methods and needs to be checked to satisfy AASHTO requirements.

Excessive cross-frame forces during the construction of steel bridge might result in the buckling of cross-frames members, however, to author's knowledge no such problems have been reported. Further, the forces in the cross-frame can also be reduced by reducing the stiffness of cross-frames or by using lean-on bracing concept that involves strategic elimination of diagonal members from different cross-frames [25].

For the Final Fit detailing method, the lack-of-fit between the cross-frames and their connections to girders appear at the erection stage. Therefore, fit-up forces are required to fit the cross-frames between their connections to girders. Calculation of the fit-up forces is explained in detail in chapter 4. Knowledge of the fit-up force is helpful regarding making arrangements for its application and the selection of a detailing method. 
Table 5.3: Structural issues related to the Erected Fit and the Final Fit detailing methods

\begin{tabular}{|c|c|c|c|}
\hline & Erected Fit & Final Fit & Comments \\
\hline Girder layovers & $\begin{array}{l}\text { Close to zero at SDL } \\
\text { stage } \\
\text { Can be significant at the } \\
\text { TDL stage }\end{array}$ & $\begin{array}{l}\text { Can be significant at the } \\
\text { SDL stage } \\
\text { Close to zero at the TDL } \\
\text { stage }\end{array}$ & $\begin{array}{l}\text { Girder layovers do not } \\
\text { have significant impact } \\
\text { on strength of the } \\
\text { structural system }\end{array}$ \\
\hline $\begin{array}{l}\text { Component of vertical } \\
\text { reaction due to lack-of- } \\
\text { fit }\left(R_{\mathrm{Y} 2}\right)\end{array}$ & $\begin{array}{l}\text { Close to zero at the SDL } \\
\text { stage } \\
\text { can be significant at the } \\
\text { TDL stage; however, no } \\
\text { uplift case is observed }\end{array}$ & $\begin{array}{l}\text { Can be significant at the } \\
\text { SDL stage and can } \\
\text { result in potential uplift } \\
\text { Close to zero at the TDL } \\
\text { stage }\end{array}$ & $\begin{array}{l}\text { Uplift observed for the } \\
\text { Final Fit at the SDL } \\
\text { stage } \\
\text { No uplift observed for } \\
\text { the Erected Fit at the } \\
\text { TDL stage }\end{array}$ \\
\hline $\begin{array}{l}\text { Component of the } \\
\text { vertical deflection due } \\
\text { to lack-of-fit }\left(D_{\mathrm{Y} 2}\right)\end{array}$ & $\begin{array}{l}\text { Permanent for the } \\
\text { Erected Fit detailing } \\
\text { method }\end{array}$ & $\begin{array}{l}\text { Temporary for the Final } \\
\text { Fit detailing method }\end{array}$ & $\begin{array}{l}\text { Taken care by } \\
\text { recommendation on } \\
\text { camber calculation }\end{array}$ \\
\hline $\begin{array}{l}\text { Flange lateral bending } \\
\text { stress }\left(f_{l}\right)\end{array}$ & $\begin{array}{l}\text { Small at the SDL stage, } \\
\text { Can be significant when } \\
\text { wet concrete is placed } \\
\text { over girders }\end{array}$ & $\begin{array}{l}\text { Can be significant at the } \\
\text { SDL stage due to pull } \\
\text { and push of flanges and } \\
\text { before casting deck } \\
\text { Close to zero after } \\
\text { completing casting the } \\
\text { deck }\end{array}$ & $\begin{array}{l}\text { Need calculation and } \\
\text { comparison to } \\
\text { AASHTO limits for } \\
\text { both detailing methods } \\
\text { however, using different } \\
\text { load combinations }\end{array}$ \\
\hline Cross-frame Forces & $\begin{array}{l}\text { Small at the SDL stage } \\
\text { Can be significant when } \\
\text { wet concrete is placed } \\
\text { over girders }\end{array}$ & $\begin{array}{l}\text { Can be significant at the } \\
\text { SDL stage due to the } \\
\text { lack-of-fit } \\
\text { Small after completing } \\
\text { casting the deck }\end{array}$ & $\begin{array}{l}\text { No field problems } \\
\text { reported for the cross- } \\
\text { frames during the } \\
\text { construction of skewed } \\
\text { bridges }\end{array}$ \\
\hline Fit-Up Forces & $\begin{array}{l}\text { Close to zero when } \\
\text { girders are under their } \\
\text { own weight (SDL stage) }\end{array}$ & $\begin{array}{l}\text { Can be significant at the } \\
\text { SDL stage because } \\
\text { cross-frames are } \\
\text { detailed to fit between } \\
\text { their connections at the } \\
\text { TDL stage and therefore } \\
\text { do not fit between their } \\
\text { connections at the SDL } \\
\text { stage }\end{array}$ & $\begin{array}{l}\text { See chapter } 4 \text { for more } \\
\text { detail description and } \\
\text { procedure for estimation }\end{array}$ \\
\hline
\end{tabular}




\subsection{Other Considerations}

There are other considerations that must be taken into account when selecting the detailing methods for skewed steel bridges. These considerations include; erection, detailing and fabrication work, inspection during the construction, and deck casting sequence.

The choice of detailing method could affect different fabricators in different ways. In the Erected Fit method the cross-frames are detailed to fit between their connections to girders, before casting the deck and after application of the steel dead load. In this case there are two alternatives: a) detail and fabricate each cross-frame differently and b) vary the location of bolt holes within stiffeners used to attach the cross-frames to girders. Some fabricators choose to fabricate each cross-frame differently and mark them appropriately for identification and installation in the field. At a first glance, this appears to increase the amount of fabrication and detailing work significantly. However, some fabricators feel this is not the case because they have automated the process. On the other hand for the Final Fit, the cross-frames are detailed to fit between their connections to girders at the TDL stage and therefore many of them are the same. Therefore, in the Final Fit method the amount of detailing and fabrication appears to be lower. However, the significance of these issues, considering the automation processes in place, depends largely on detailers and fabricator's capabilities.

With respect to erection, the Erected Fit method is simpler as compared to the Final Fit method. This is because for the Erected Fit method, cross-frames are detailed to fit between their connections to girders at the SDL stage, while fit-up forces are required to fit the cross-frames between their connections to girders in the case of the Final Fit 
method. In extreme cases, the level of fit-up forces could be significant for the Final Fit method [2] [3].

Another consideration that deserves attention is the sequence of deck casting. There are two separate "deck casting" sequence considerations. First related to phased construction, where the bridge is constructed in "phases" and different phases are connected together using closure pours and cross-frames. The choice of the detailing method in this case requires specific analysis and no general statement can be made. The second case is when the deck casting sequence is used to minimize the cracking of the deck during the casting. In multi-span bridges, the positive moment sections are generally casted simultaneously, followed by casting the remaining negative moment sections. The recommendation is to ensure that all cross-frames are first attached, prior to doing any deck casting, which is the practice norm.

Some contractors have raised a concern about the inspection. The webs of the girders are out of plumb for the Final Fit method at the SDL stage and at the TDL stage for the Erected Fit detailing method. This behavior needs to be communicated with the owner, erector and contractor to avoid miscommunications. It should also be noted that even in the case of the Final Fit detailing method the web could be somewhat out of plumb at the TDL stage and should not be a point of concern, as the web out of plumpness does not affect the structural response of skewed steel bridges, significantly.

The problems discussed above are related to the construction of skewed bridges. Very few problems are reported in skewed bridges once the construction is complete. One such problem is fatigue cracks from large stress concentrations in the girder due to 
cross-frame and diaphragm forces induced by the truck traffic in bridges with skewed supports. These problems are addressed by improving the bracing details [11] [13].

\subsection{Parametric Studies}

Parametric studies are carried to evaluate different options a designer might consider for the framing of a straight skewed I-girder bridge. These parametric studies include the followings:

- Effect of the cross-frame stiffness.

- Effect of the distance of first intermediate cross-frame from the support.

- Effect of the cross-frame orientation.

\subsubsection{Effect of Cross-frame Stiffness}

Cross-frame forces are affected by equivalent areas of cross-frame members $\left(A_{b}\right)$ considered in the FEM modeling of the bridge. Typical single-angle or structural Tee cross-frame members are subjected to additional bending deformations due to the eccentricity of their end connections normal to the connection and/or gusset plates. The corresponding bending deformations reduce the stiffness of the cross-frame members,

and hence the overall stiffness of the cross-frames [31]. In this research, the effect of reducing the equivalent area of the cross-frame members on cross-frame forces is studied. Cross-frame forces in the top chord of cross-frames in Bay 7 of Bridge A for different areas of cross-frame members for the Erected Fit detailing method at the TDL stage are shown in Figure 5.7. The results shown in Figure 5.7are obtained from the 3D FEM analysis with flanges modeled with shell elements; however, similar results are obtained from other methods of analysis. From Figure 5.7following observations are made 
- The cross-frame forces are reduced by reducing the cross section area of crossframe members.

- The reduction in force varies significantly between different cross-frame members because redistribution of the force occurs by reducing the cross-frame area.

Similar observations were observed for other bridges analyzed as part of this study.

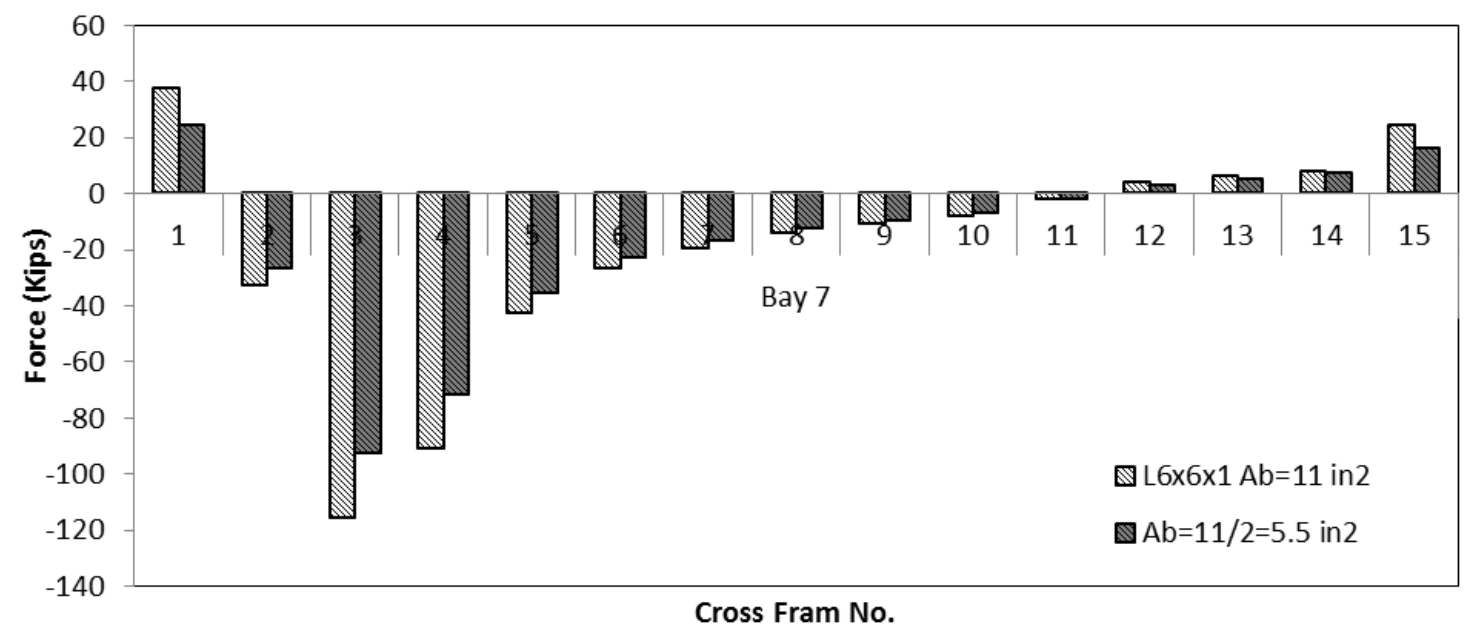

Figure 5.7: Effect of reducing the area of cross member on cross-frame forces in Bridge A-Erected Fit at the TDL stage

Table 5.4 provides summary of the reduction in the maximum cross-frame force by reducing the area of cross-frame members by half for Bridge A, Bridge B and Bridge C. As shown in Table 5.4 the maximum cross-frame force does not reduce significantly by reducing the area of cross-frame member to half for bridges studied.

Table 5.4: Summary of effect area of cross-frame members on cross-frame forces

\begin{tabular}{lrrrrrr}
\hline & \multicolumn{3}{c}{ Bridge A } & \multicolumn{2}{c}{ Bridge B } & \multicolumn{2}{c}{ Bridge C } \\
\cline { 2 - 6 } Area of cross-frame members $\left(\right.$ in $\left.^{2}\right)$ & 11 & 5.5 & 5.75 & 2.875 & 2.87 & 1.435 \\
Maximum Absolute cross-frame force (kips) & 115.6 & 92.9 & 38.5 & 24.4 & 20.3 & 14.5 \\
\hline
\end{tabular}




\subsubsection{Effect of the Distance of the First Intermediate Cross-frame from Support}

Field observations indicate that the distance between support and the first intermediate cross-frame has significant influence on vertical reactions and cross frame forces during the erection of skewed steel I-girder bridges. To study the this parameter, framing plan of Bridge A was changed to create several framing plans that are identical, except the distance between the support and the first intermediate cross-frame, as shown in Figure 5.8. This is achieved by varying the distance between support and first crossframe by $6 \mathrm{ft}$. increment, while keeping the spacing between other cross-frames at constant $26 \mathrm{ft}$.

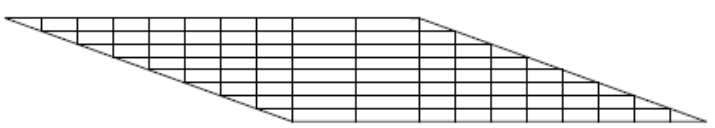

$0 \mathrm{ft}$. from support

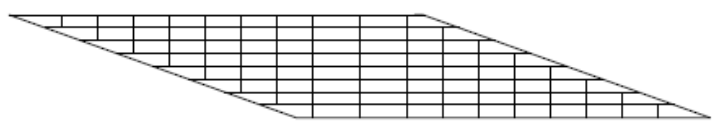

$12 \mathrm{ft}$. from support

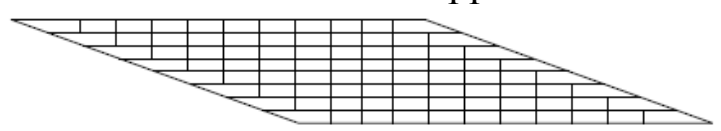

$24 \mathrm{ft}$. from support

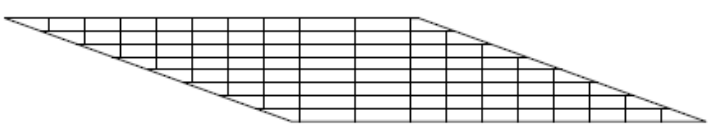

$6 \mathrm{ft}$. from support

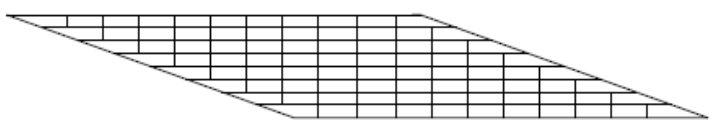

$18 \mathrm{ft}$. from support

Figure 5.8: Framing plans to study effects of the location of the first intermediate cross-frame from the support in Bridge A

Different structural responses, such as girder layovers, cross-frame forces, and reactions were evaluated through the 3D FEM analysis for the Erected Fit detailing method at the TDL stage for framing plans shown in Figure 5.8. Out of different structural responses, cross-frame forces appear to be most affected by varying the distance between the support and the first cross-frame as shown in Figure 5.8. Crossframe forces decrease with the increase in the distance of the first intermediate cross- 
frame from the support. However, the total change in cross-frame force is about $25 \%$ for changing the location of the first intermediate cross-frame from the support from $0 \mathrm{ft}$ to 24ft. The distance between the support and the first cross-frame does not appear to significantly influence the vertical reactions, as shown in Figure 5.9. Similarly, the results of this study also show that girder layovers are not affected significantly by the distance between support and the first cross-frame.
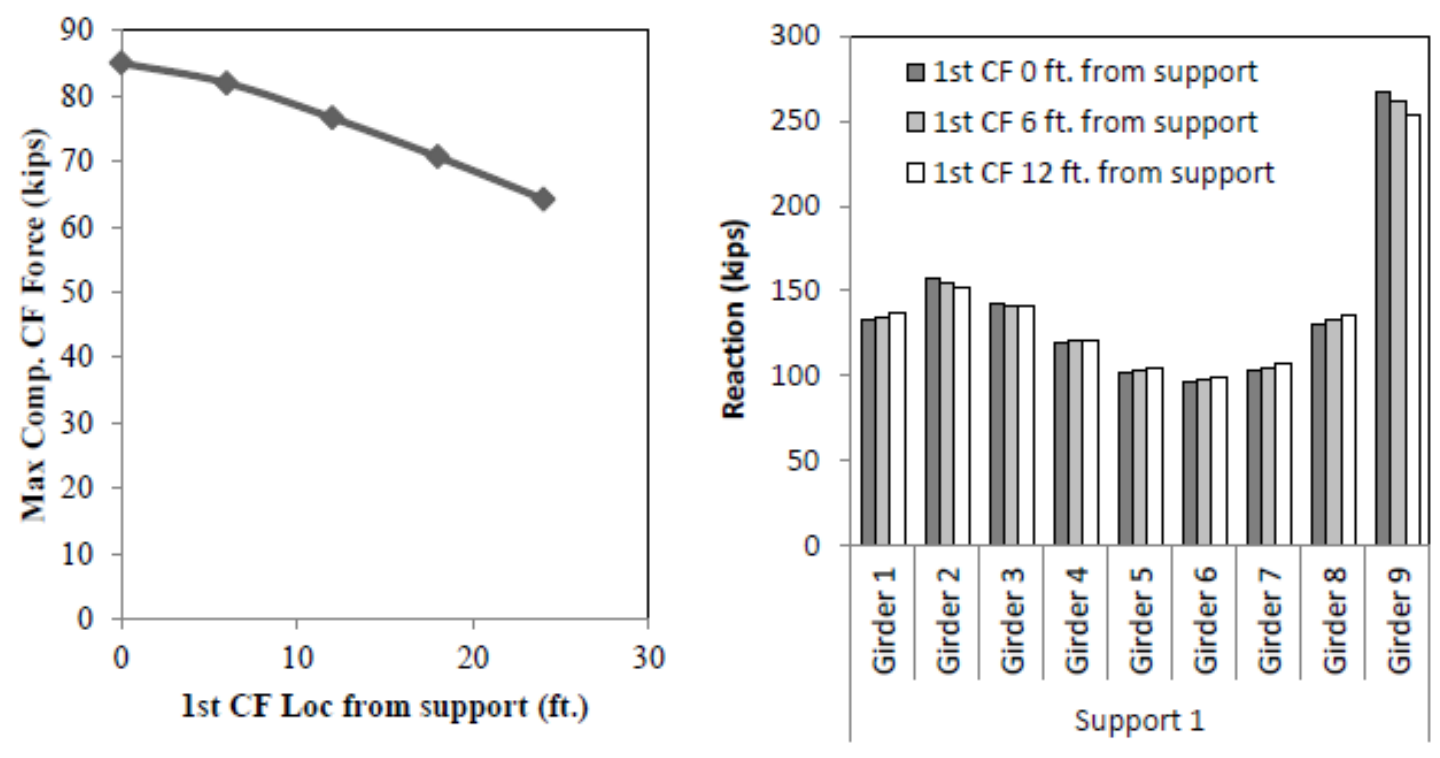

Figure 5.9: Variation of structural responses by changing the location of first intermediate cross-frame from the support in Bridge A- Erected Fit detailing under the TDL

Effects of the distance between the support and the first intermediate cross-frame need to be evaluated for a continuous bridge because the cross-frames on the opposite sides of the continuous support in a continuous bridge twist girders in opposite directions and that can magnify certain structural responses. 
A typical two span (150 ft. and $140 \mathrm{ft}$.) continuous bridge with a skew angle of $60^{\circ}$ at all supports is selected to study the effects of distance between support and first intermediate cross-frame. The bridge has six 54 inch deep girders spaced at $8.2 \mathrm{ft} . \mathrm{c} / \mathrm{c}$. Top and bottom flanges are 18 inch wide with varying thickness along the length of the girder $(1$ inch thick from $0 \mathrm{ft}$. to $88 \mathrm{ft}$., 1.75 inch thick from $89 \mathrm{ft}$. to $118 \mathrm{ft}$., 2.75 inch thick from $119 \mathrm{ft}$. to $176 \mathrm{ft}$., 1.75 inch thick from $177 \mathrm{ft}$. to $206 \mathrm{ft}$., and 1 inch thick from 207ft. to 290ft.).The girders of the bridge are braced with X-type cross-frames containing L4 $x 4 \times 3 / 8$ angles. The bridge uses cross-frames at spacing of $14 \mathrm{ft}$. near the support. Framing planes of the bridge used for this study are shown in Figure 5.10.

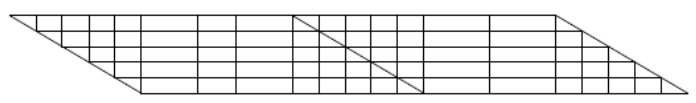

$0 \mathrm{ft}$. from support

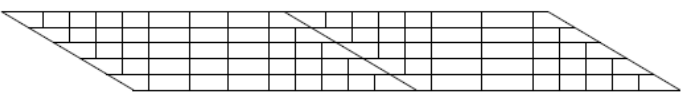

$8 \mathrm{ft}$. from support

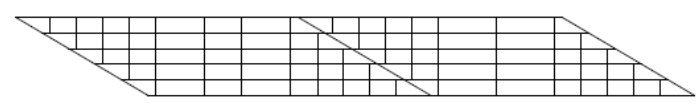

$4 \mathrm{ft}$. from support

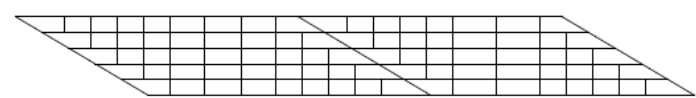

$12 \mathrm{ft}$. from support

Figure 5.10: Framing planes to study effects of location of $1^{\text {st }}$ intermediate crossframe from the support in a continuous bridge

Different structural responses such as, girder layovers, cross-frame forces, and vertical reactions are evaluated through the 3D FEM analysis for the Erected Fit detailing method at the TDL stage for the framing plans shown in Figure 5.10.

Out of the different structural responses, cross-frame forces appear to be the most affected by varying the distance between the support and the first cross-frame, as shown in Figure 5.11. Cross-frame forces decrease with increase in the distance between the first intermediate cross-frame and the support. However, total change in cross-frame force is 
about $30 \%$ for changing the location of the first intermediate cross-frame from the support from $0 \mathrm{ft}$. to $12 \mathrm{ft}$. The influence of distance between support and first cross-frame does not appear to significantly influence the vertical reactions, as shown in Figure 5.12. Similarly, results of this study also shows that girder layovers are not affected significantly by distance between support and first cross-frame.

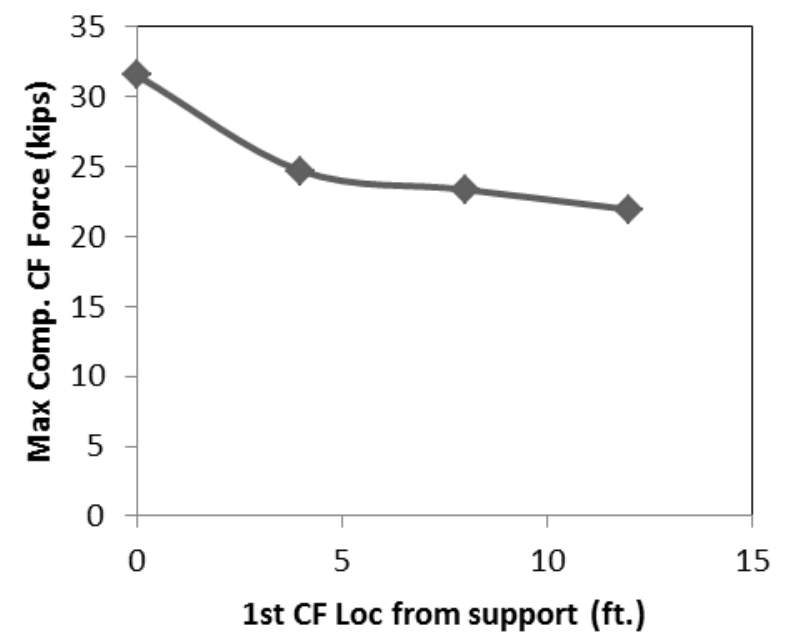

Figure 5.11: Variation of cross-frame forces by changing location of the first intermediate cross-frame from the support-Erected Fit at the TDL stage

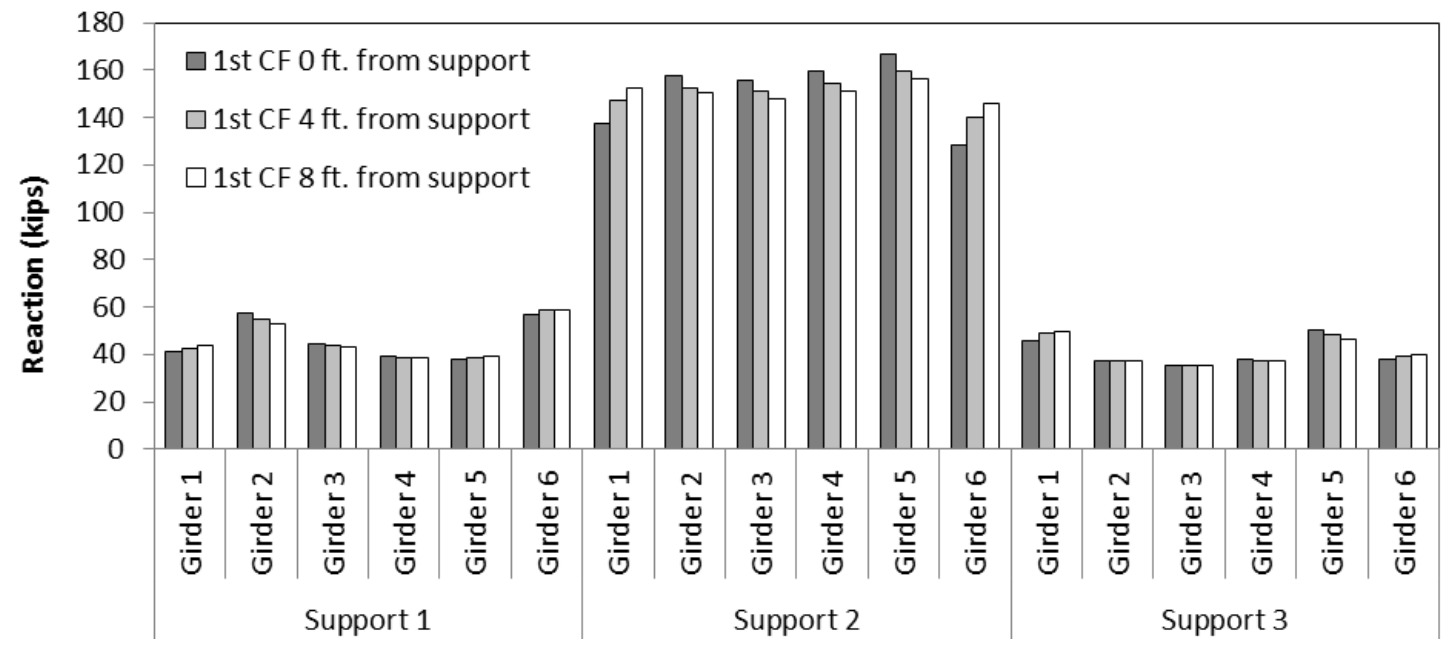

Figure 5.12: Variation of vertical reactions by changing the location of the first intermediate cross-frame from the support-Erected Fit at the TDL stage 


\subsubsection{Effect of Cross-frame Orientation}

Different cross-frame options are normally used to mitigate girder layovers in straight skewed I-girder bridges. Comparison of two such framing options is done here for Bridge A. In framing plan 1, cross-frame are attached perpendicular to the girder web and are staggered along the length of the bridge as shown in Figure 5.13(a). The framing plane 2 has cross-frames placed parallel to skewed supports with typical cross-frame spacing of 20ft as shown in Figure 5.13(b).

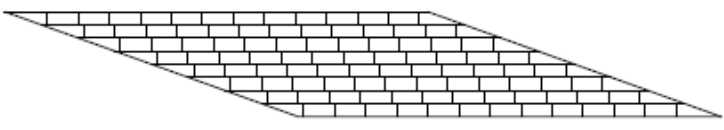

(a) Framing Plan 1

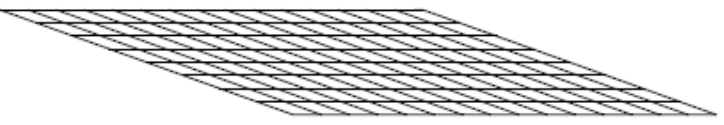

(b) Framing Plan 2

\section{Figure 5.13: Different cross-frame orientations}

As discussed in chapter 2 there are two major sources of the twist in the straight skewed I-girder bridges and these are the differential deflection of the points attached by cross-frames perpendicular to the web and the rotation of cross-frames parallel to the skew. In framing plan 1 , girder layovers appear due to both the differential deflection and the rotation of cross-frames parallel to skew, whereas in framing plan 2, girder layovers appear only from the rotation of cross-frames parallel to skew. For these cases girder layovers are compared for the Erected Fit detailing method at the TDL stage.

Comparison of girder layovers obtained for different framing options are shown for Girder 1 and Girder 5 of Bridge A in Figure 5.14. In Girder 1 girder layovers are higher for framing plan 1 compared to girder layovers obtained for framing plan 2. For Girder 5 girder layovers are less for framing plane 1 compared to framing plane 2. In both case the difference in girder layovers is not that significant. Results of the study 
indicate that orienting the cross-frame parallel to skew does not significantly reduce girder layovers. When cross-frames are parallel to skew, they still cause twist in the girder; because, the axis of rotation of these cross-frames is not parallel to the major axis bending axis of rotation of girders.

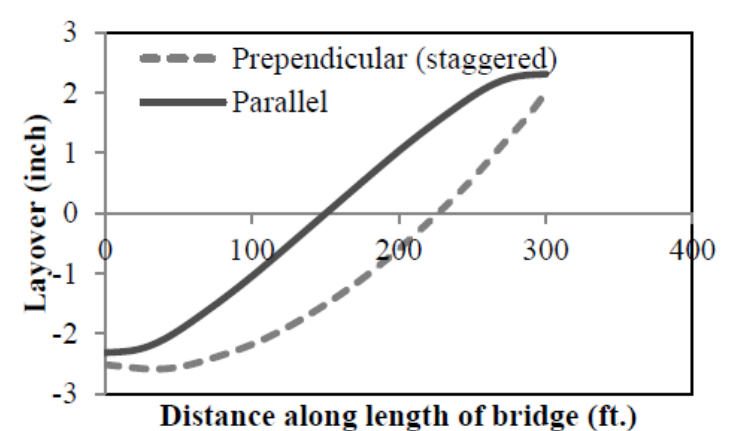

(a) Girder 1

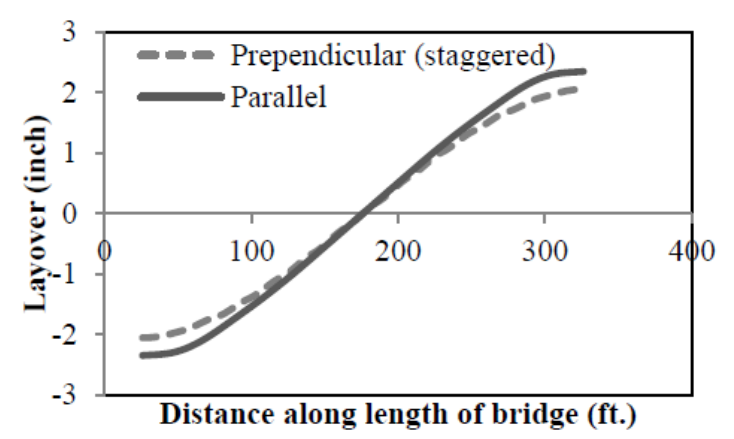

(b) Girder 5

Figure 5.14: Layovers for different cross-frame orientations-Erected Fit at the TDL stage

\subsection{Flow Chart for Design}

Two flow charts, one for each detailing method, are proposed to leave the ultimate choice of detailing method to owners and designers. The selection of the detailing method depends on many factors and the final choice that could be influenced by several factors such as, local practices and the owner, designer, fabricator and erector preferences. However, a flow chart is developed for each detailing method, as shown in Figure 5.15 to facilitate the selection of detailing method.

Flange lateral bending stress $\left(f_{l}\right)$ needs to be checked for both Final Fit and Erected Fit detailing methods to satisfy the AASHTO bridge design requirements. For the Final Fit detailing method, $f_{l}$ at the SDL stage comes from the lack-of-fit and the wind load. For the Erected Fit detailing method $f_{l}$ at the TDL stage comes from the lack-of-fit 
and knee braces. AASHTO LRFD Bridge Design Specifications should be used for appropriate load combinations to check the level of flange lateral bending stresses. There may be a need to increase the flange sizes, which may dictate the choice of the detailing method.

In the Final-Fit method the additional structural response that needs to be calculated is the maximum fit-up force required for fitting the cross-frames between their connections to girders during the erection. The knowledge of fit-up forces will allow erector to assess the need for having special equipment for fitting cross-frames between their connections to girders.
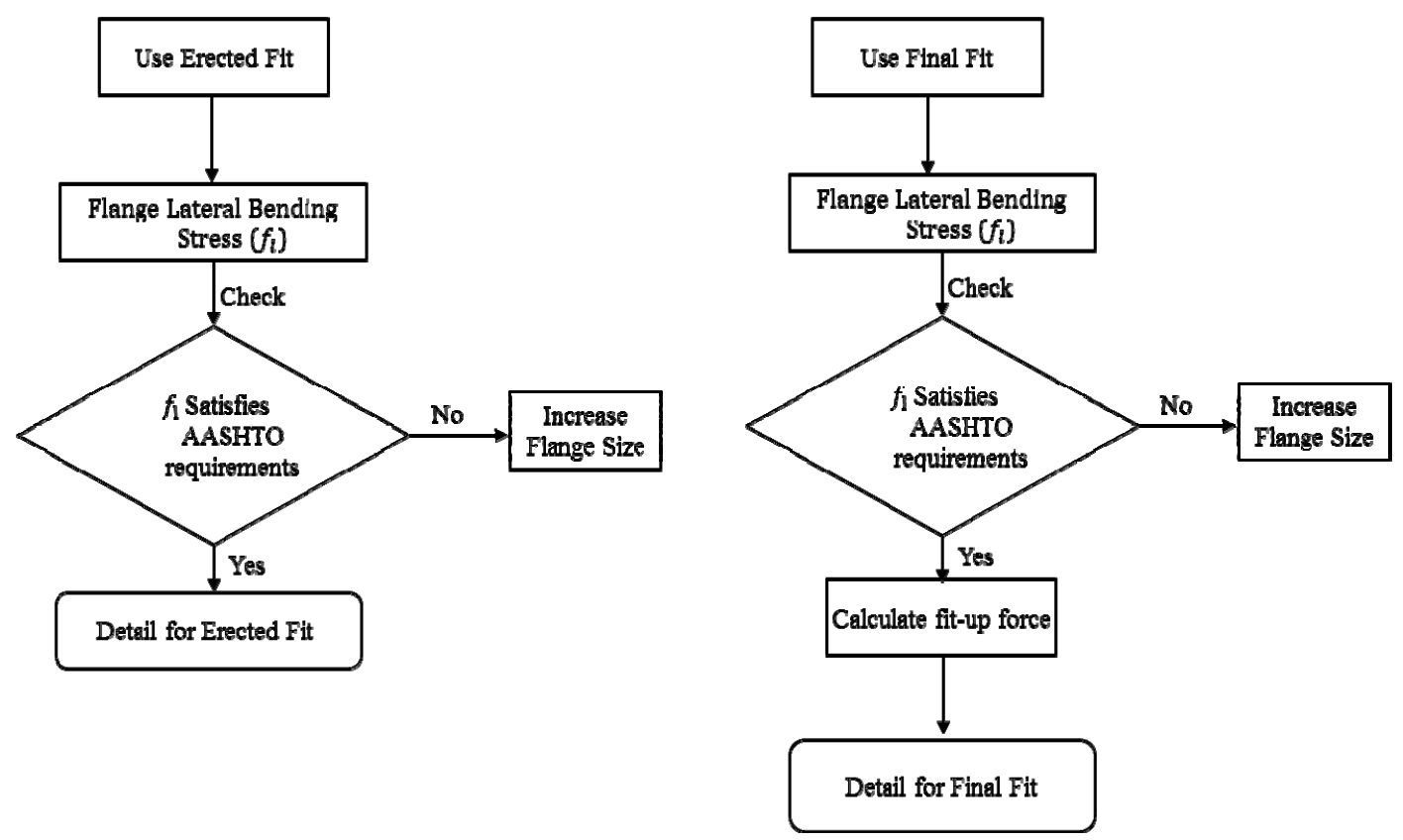

Figure 5.15: Flow chart to guide designer to deal with skewed bridges

\subsection{Summary}

This chapter provides recommendation to calculate the cambers for the erected fit and final fit detailing methods. For the erected fit detailing method, the SDL cambers should be estimated by LGA, and CDL cambers should be calculated by 2D GA or 3D 
FEM analysis modeling all the girders and cross-frames connected together. For the final fit detailing method, both SDL and CDL cambers need to be calculated by LGA.

The following conclusions could be made from these limited analyses:

- The recommended procedure does a good job of predicting the camber for the final fit detailing method.

- When cross-frames are staggered, or for odd cases where one would be suspicious about the accuracy of 2D GA, camber needs to be calculated using refined methods of analysis.

This chapter also provides a summary of lack-of-fit effects in skewed and straight steel girder bridges and related field challenges. Important lack-of-fit effects that need to be checked for a particular detailing method are identified. A summary of other construction issues, such as detailing and fabrication, deck casting sequence, and inspection during erection is also provided. Parametric studies have shown that the distance of the first intermediate cross-frame from the support, and reducing the cross section area of cross-frame members do not have significant effect on cross-frame forces and other lack-of-fit effects. Further, arranging the cross-frames parallel to skewed supports or perpendicular to the girders does not change the maximum value of layover significantly. Finally, a flowchart is developed for each detailing method to facilitate the selection of detailing method and shows the necessary design calculations that need be carried out. 


\section{CONCLUSIONS AND RECOMMENDATIONS}

The most important conclusion of this research is that lack-of-fit effects for the Final Fit detailing method at the steel dead load stage are equal and opposite to the lackof-fit effects for the Erected Fit detailing at the total dead load stage. These lack-of-fit effects include the following:

- Girder layovers

- Flange lateral bending stress

- Cross-frame forces

- Component of vertical deflection due to lack-of-fit

- Component of vertical reaction due to lack-of-fit

- Lateral reactions/movements

Girder layovers at the bearing pads also indicated the additional rotations at the bearing pad. The conclusions and recommendations discussed in this chapter are divided in different topics covered in this dissertation.

\subsection{Detailing Methods}

The major finding on detailing methods is that lack-of-fit effects for the Final Fit detailing method at the SDL stage are equal and opposite to the lack-of-fit effects for the Erected Fit detailing method at the TDL stage.

Vertical reactions and vertical deflections are divided into two components. One component is from the dead loads and can be evaluated from the line or isolated girder analysis. The other component comes from the lack-of-fit of cross-frames between their connections to girders and be estimated by subtracting the dead load component from the total vertical reactions or deflections. 
The mechanism by which lack-of-effects develop in the skewed bridges for a particular detailing method at a particular loading stage is explained. It has been found that dead loads move the girders to different positions and cross-frames fit between the girders at a particular position depending on the detailing method used. Lack-of-fit effects develop after attaching cross-frames to girders at a loading stage at which there is a lack-of-fit (distance between the cross-frames and their connection points before connecting cross-frames). The lack-of-fit effects are proportional to the lack-of-fit for given bridge geometry and member sizes.

\subsection{Method of analysis}

2D grid analysis methods have been used for no load fit detailing method only in the past and 3D FEM analyses with initial strains are required for dead load detailing methods (Erected Fit and Final Fit). The conclusions made in chapter 2 of this dissertation provide the foundation for using 2D grid analysis methods for dead load detailing methods. Different 2D grid analysis methods such as, the traditional 2D grid analysis and improved 2D grid analysis, are evaluated for calculating lack-of-fit effects for the Erected Fit and Final Fit detailing method. It has been found that performance of improved and traditional 2D grid analysis is different for staggered cross-frames and contiguous cross-frames.

- For bridges with contiguous cross-frames, the traditional 2D GA gives reasonable estimates of all lack-of-fit effects except for the flange lateral bending stress and the improved 2D GA gives reasonable estimates of all lack-of-fit effects.

- For bridges with staggered cross-frame, the traditional 2D GA gives erroneous estimates of all lack-of-fit effects and the improved 2D GA gives reasonable estimates of all lack-of-fit effects. However, when the stagger distance is small, 
$J_{e q}$ in the improved 2D GA gets very high value resulting in the overestimation of lack-of-fit effects.

For the Final Fit detailing method, two new methods of analysis are introduced for calculating lack-of-fit effects at the SDL stage. These methods are: a) using the 3D FEM with dead and live cross-frame elements and b) reversing the improved 2D grid analysis results for the Erected Fit detailing method at the TDL stage.

Lack-of-fit effects for the Final Fit detailing method at the SDL stage obtained from the method of initial strains shows a very good agreement with the lack-of-fit effects obtained from the method of Dead and Live cross-frames elements. Reversing the improved 2D grid analysis results for the Erected Fit detailing method at the TDL stage also give reasonable estimates of lack-of-fit effects for the Final Fit detailing method at the SDL stage.

The main conclusion on methods of analysis is that the improved 2D GA can be used to estimate the lack-of-fit effects for both Final Fit and Erected Fit detailing method.

\subsection{Fit-up forces}

Fit-up forces are required for attaching the cross-frames during the erection in the case of Final Fit detailing method. The knowledge of fit-up forces will allow erectors to mobilize the required equipment on site to avoid job delays.

Two different methods are introduced to evaluate the maximum fit-up force. These methods are the cross-frame forces method and the 3D erection simulation method. It has been shown that cross-frame forces evaluated from the improved 2D grid analysis can be used to estimate the maximum fit-up force required to fit the cross-frames between their connections to girders during the erection of a steel bridge. 
Three different erection sequences have been used in the erection simulation method to find out the most efficient sequence of erection. It has been found that the maximum fit-up force required to erect the cross-frames is relatively less if cross-frames are erected starting from the middle of a bay and moving toward the ends of the bay (erection sequence 3 ).

\subsection{Design Recommendation}

It is recommended to calculate the cambers for the Erected Fit and the Final Fit detailing methods using the line girder analysis or the combination the line girder analysis and the 2D grid analysis. For the Erected Fit detailing method, the SDL cambers should be estimated by the line girder analysis and the concrete dead load (CDL) cambers should be calculated by the 2D grid analysis or the 3D FEM analysis modeling all the girders and cross-frames connected together. For the Final Fit detailing method both the SDL and CDL cambers need to be calculated by the line girder analysis.

From the limited numerical analysis following conclusions could be made:

1- The recommended procedure does a good job of predicting the camber for the Erected Fit and Final Fit detailing method.

2- When cross-frames are staggered or for odd cases, where one would be suspicious about accuracy of 2D grid analysis, camber needs to be calculated using refined methods of analysis.

Lack-of-fit effects in straight skewed steel I-girder bridges and related field challenges are summarized. Important lack-of-fit effects that need to be checked for a particular detailing method are identified. Summary of other construction issues such as, 
the detailing and fabrication, deck casting sequences, and inspection during the erection is also provided.

Parametric studies are carried out to evaluate different design options related to skewed bridges. These studies have shown that the distance between the first intermediate cross-frame and the support, and reducing the cross section area of crossframe members do not have significant effect on the cross-frame forces and other lack-offit effects. Further, arranging the cross-frames parallel to skewed supports or perpendicular to the girder webs do not change the maximum value of layovers significantly. Finally, a flowchart is developed for each detailing method to facilitate the selection of the detailing method and carrying the necessary design calculations.

\subsection{Future Research}

This dissertation provides a comprehensive study of detailing methods used for detailing the cross-frame and girders in straight skewed steel I-girder bridges. Steel bridges are designed to be in linear constitutive range during different stages of their construction. The finite element analysis generally gives very good estimate of structural responses when materials have linear constitutive relations.

There have been few field studies conducted to measure limited structural responses; however, a detailed field study to comprehend the detailing methods is not yet carried out. One problem with the field study is that in real bridges cross-frames are detailed by using one particular detailing method, therefore comparisons cannot be made for the same bridge using the other detailing methods. Further, once concrete dead load is 
applied by pouring the deck, it cannot be removed to see bridge configuration at the SDL stage.

This problem can be solved by carrying out different test on a small scale steel bridge specimen having different sets of cross-frames detailed with the different detailing methods. A load, equivalent to the load of fresh concrete in deck, can be applied and removed by using sand or other material. This will enable to compare different detailing methods at different loading stages. The tests can be used to complement the numerical studies carried out to comprehend the behavior of cross-frame in steel skewed bridges. 


\section{REFERENCES}

[1] Turco, G. (2009). Field Retrofitting a Curved Girder Bridge to Increase Torsional Stiffness. Presentation at Proceeding of National Steel Bridge Alliance 2009 World Steel BridgeSymposium, San Antonio, Texas, November 17-20 .

[2] Beckmann, F. R., \& Mertz, D. R. (2005). Steel Bridge Erection Practices. Washington, D.C.: Transportation Research Board.

[3] AASHTO. (2012). AASHTO LRFD Bridge Design Specifications. Washington, DC: American Association of State Highway and Transportation Officials.

[4] Morera, F. J. (2010). Lateral Flange Bending in Heavily Skewed Steel Bridges. Doctoral Dissertation . Raleigh, NC: North Carolina State University.

[5] Ahmadi, A. K., \& Henney, R. (2005). Lessons Learned from the Construction of a Sharply Skewed, Two-span, Steel MultI-girder Bridge. International Bridge Conference (p. 8). Hilton Pittsburgh: International Bridge Conference IBC.

[6] Beckmann, \& Medlock. (2005). Skewed Bridges and Girder Movements Due to Rotations and Differential Deflections. Proceeding 2005 World Steel Bridge. Symposium and Workshops. November 29 - December 2.

[7] Grunauer, T. A. (2011, December). Influence of bracing systems on the behaviour of curved and skewed steel I-Girder Bridges during construction. PhD thesis . Georgia Institute of Technology.

[8] Ozgur, C. (2011, December ). Influence of Cross frame detailing on curved and skewed steel I-Girder Bridges. PhD Thesis . Georgia Institute of Technology.

[9] White, D. W., Coletti, D., Chavel, B. W., Sanchez, A., Ozgur, C., Chong, J. M., et al. (2012). NCHRP 725: Guidelines for Analysis Methods and Construction Engineering of Curved and Skewed Steel Girder Bridges. NCHRP and NSBA.

[10] AASHTO/NSBA, C. S. (2011). G 13.1 Guidlines for steel girder bridge analysis. AASHTO/NSBA Steel Bridge Collaboration.

[11] Linzell, D., Chen, C., Sharafbayani, M., Seo, J., Nevling, D., Jaissa-Ard, T., et al. (2010). Guidelines for Analyzing Curved and Skewed Bridges and Designing them for Construction. Harrisburge, PA: Pennsylvania Department of Transportation.

[12] Linzell, D., Abner Chen, M. S., Seo, J., Nevling, D., Jaissa-Ard, T., \& Ashour, O. (2010). Guidelines for Analyzing Curved and Skewed Bridges and Designing Them for Construction. Harrisburg: Pennsylvania Department of Transportation. 
[13] Fisher, S. (2006). Development of a Simplified Procedure to Predict Dead Load Deflections of Skewed and Non-Skewed Steel Plate Girder Bridge. M.S. Thesis . School of Civil Engineering, North Carolina State University, Raleigh, NC.

[14] Whisenhunt, T. (2004). Measurements and Finite Element Modeling of the NonComposite Deflections of Steel Plate Girder Bridges. M.S. Thesis. School of Civil Engineering, North Carolina State University, Raleigh, NC.

[15] Coletti, D., \& Yadlosky, J. (2008). Analysis of Steel Girder Bridges: New Challenges. Journal of the Transportation Research Board, 67-77.

[16] Changa, C.-J., \& White, D. W. (2008). An assessment of modeling strategies for composite curved steel I-girder bridges. Engineering Structures, Volume 30, Issue 11 , 2991-3002.

[17] Ahmed, M., \& Weisgerber, F. (1996). Torsion Constant for Matrix Analysis of Structures Including Warping Effect. International Journal of Solids and Structures, Elsevier 33(3), 361-374.

[18] Chavel, B. W. (2008). Construction and Detailing Methods of Horizontally Curved Steel I-Girder Bridges. Pittsburgh, PA: Doctoral Dissertation, University of Pittsburgh.

[19] Bell, B., \& Linzell, D. (2007). Erection Procedure Effects on Deformations and Stresses in a Large-Radius, Horizontally Curved, I-Girder Bridge. Journal of Bridge Engineering, 12(4), 467-476.

[20] Chang, C. (2006). Construction Simulation of Curved Steel I-Girder Bridges. PhD thesis . Georgia Institute of Technology, Atlanta, GA.

[21] Chang, C., \& White, D. (2006). Construction Simulation of Curved I-Girder Bridge Systems. Annual Proceedings, Structural Stability Research Council, San Antonio, TX, 93-114.

[22] Chavel, B. W., \& Earls, C. J. (2006). Construction of a Horizontally Curved Steel IGirder Bridge. Part I: Erection Sequence. Journal of Bridge Engineering , 81-90.

[23] Wang, W. (2013). A Study of Stiffness of Steel Bridge Cross Frames. Doctoral Dissertation. The University of Texas at Austin.

[24] Battistini, A. D., Donahue, S., Wang, W. H., Helwig, T. A., Engelhardt, M. D., \& Frank, K. H. (2013). Brace Stiffness and Forces of X Type, K-Type, and Z-Type Cross Frames in Steel I-Girder Bridge System. Proceedings of Structural Stability Research Council/North American Steel Construction Conference, St. Louis, MO, April 16-20, 2013.

[25] Battistini, A. D., Wang, W. H., Helwig, T. A., Engelhardt, M. D., \& Frank, K. H. (2012). Comparison of the Stiffness Properties for Various Cross Frame Members and 
Connections. Proceedings of Structural Stability Research Council/North American Steel Construction Conference, Grapevine, TX, April 17-21, 2012.

[26] Liqun, W., \& A., H. T. (2008). Stability Bracing Requirements for Steel Bridge Girders with Skewed Supports. SCE Journal of Bridge Engineering, Vol. 13, No. 2 , 149157.

[27] Quadrato, C. E., Battistini, A. D., Helwig, T. A., Engelhardt, M. D., \& Frank, K. H. (2009). The Impact of Connection Flexibility on the Brace Stiffness Requirements of Skewed Steel Bridges. Proceedings of the Structural Stability Research Council Annual Stability Conference April 1-4, 2009, (pp. 365-384). Phoenix, AZ.

[28] Zhou, C. (2006). Utilizing Lean-on Cross-Frame Bracing for Steel Bridges. MS Thesis . University of Houston.

[29] Herman, R. S., Helwig, T. A., \& Zhou, C. (2007). Use of Lean-On Cross-Frame Bracing in Steel Girder Bridges. New Horizons and Better Practices (pp. 1-6). ASCE.

[30] ANSYS. (2009). ANSYS Release 12.1 UP20091102. Analysis Software . Copyright 2009 SAS IP, ANSYS Inc.

[31] MathWorks. (2012, 11 11). MATLAB R2012b version 8.0.

[32] Norton, E., Linzell, D., \& Laman, J. (2003). Examination of the Response of a Skewed Steel Bridge Superstructure During Deck Placement. Journal of the Transportation Research Board No. 1845 , 66-75.

[33] Ozgur, C., \& White, D. (2007). Behavior and Analysis of a Curved Steel I-Girder Bridge. Proceedings World Steel Bridge Symposium . Chicago, IL: National Steel Bridge Alliance, $18 \mathrm{pp}$.

[34] Chavel, B. W., \& Earls, C. J. (2006). Construction of a horizontally curved steel Igirder bridge: Inconsistent detailing. Journal of Bridge Engineering, Vol. 11, No. 1, 9198.

[35] AASHTO/NSBA. (2007). Steel Bridge Erection Guide Specification. AASHTO/NSBA Steel Bridge Collaboration, American Association of State Highway and Transportation Officials, Washington, DC and National Steel Bridge Alliance, Chicago, IL.

[36] White, D., \& Jung, S.-K. (2007). Effect of Web Distortion on the Buckling Strength of Noncomposite Discretely-Braced I-Beams. Engineering Structures, Elsevier, 29, 1872-1888.

[37] ANSYS Inc. (2005, August). ANSYS Advanced Analysis Techniques Guide. ANSYS Inc. 
[38] ANSYS Inc. (2012, October). ANSYS Mechanical APDL Verification Manual. ANSYS, Inc. .

[39] Romage, M. L. (2008). Field Measurements on Lean-On-Bracing for Steel Girder Bridges with Skewed Supports. Masters Thesis . University of Texas at Austin, Austin, TX.

[40] Helwig, T. A., \& Wang, L. (2003). Cross-Frame and Diaphragm Behavior for Steel Bridges with Skewed Supports. Houston, TX: University of Houston.

[41] Berglund, E., \& Schultz, A. (2006). Girder Differential Deflection and DistortionInduced Fatigue in Skewed Steel Bridges. Journal of Bridge Engineering, ASCE,11(2), $169-177$.

[42] Wang, W. H., Battistini, A. D., Helwig, T. A., Engelhardt, M. D., \& Frank, K. H. (2012). Cross Frame Stiffness Study by Using Full Size Laboratory Test and Computer Models. Proceedings of Structural Stability Research Council/North American Steel Construction Conference, Grapevine, TX, April 17-21, 2012. 
APPENDICES 


\section{APPENDIX A DESCRIPTION OF BRIDGES AND LOADING}

\section{A.1 Detail of Bridges}

Three straight skewed, simply supported I-girder bridges, having different levels of skew, are selected for consideration in this study. All three bridges have their girders and cross frames designed with Grade 50 steel having a modulus of elasticity of 29,000 ksi.

Bridge ' $A$ ' is an extreme case of straight skew bridges and is used to show extreme skew effects in previous studies [1] [2] [3]. Bridge A has $300 \mathrm{ft}$. long 144 inches deep girders simply supported on $70.4^{\circ}$ skewed supports. The girders of Bridge A are braced with X-type cross frames containing L6 x 6 x 1 angles. The bridge uses staggered cross frames at spacing of $22 \mathrm{ft}$. between 9 girders at $9.25 \mathrm{ft}$. c/c spacing. Framing plans and sizes of the web and flanges of the bridges studied are shown in Figure A.1.

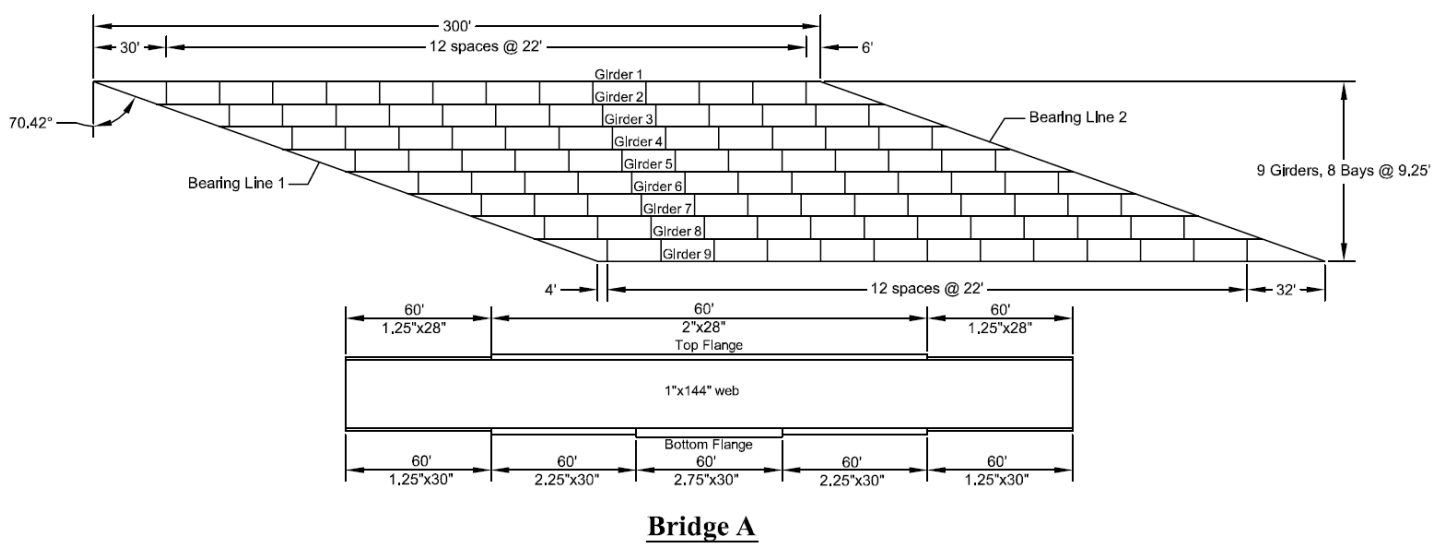

Figure A.1: Framing plans and girder sizes of the Bridge A

Bridge ' $\mathrm{B}$ ' is another highly skewed bridge, however skewed effect in Bridge $\mathrm{B}$ are smaller compared to Bridge 'A'. Bridge 'B' has $266 \mathrm{ft}$. long 120.5 inches deep girders 
simply supported on $62.6^{\circ}$ skewed supports. The girders of the Bridge B are braced with $\mathrm{X}$-type cross frames containing L6 $66 \times 1 / 2$ angles. The bridge uses cross frames at spacing of $16 \mathrm{ft}$. between 8 girders@7.26 ft. c/c spacing. Framing plans and sizes of the web and flanges of the bridges studied are shown in Figure A.2.

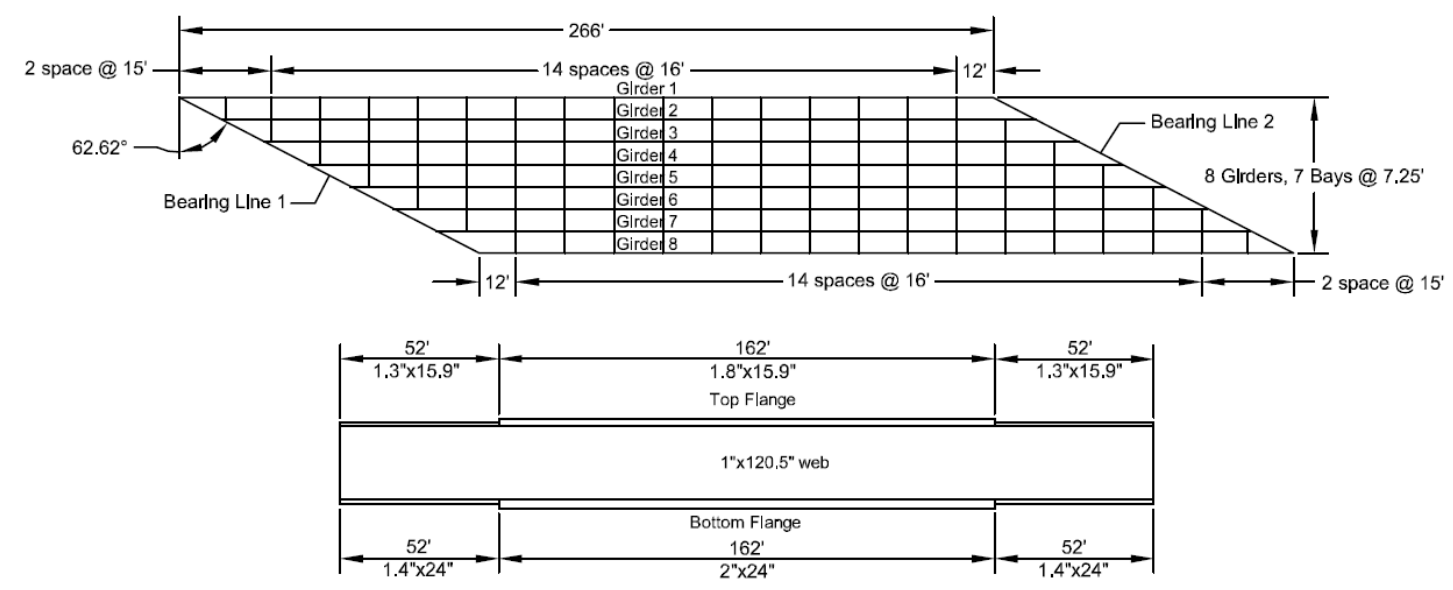

Bridge B

Figure A.2: Framing plans and girder sizes of the Bridge B

Bridge ' $\mathrm{C}$ ' has $150 \mathrm{ft}$. long 56.1 inches deep girders simply supported on $70.0^{\circ}$ skewed supports. The girders of the Bridge $\mathrm{C}$ are braced with X-type cross frames containing L6 x $31 / 2 \times 5 / 16$ angles. The bridge uses cross frames at spacing of $21 \mathrm{ft}$. between 4 girders@8ft. c/c spacing. Framing plans and sizes of the web and flanges of the bridges studied are shown in Figure A.3. 


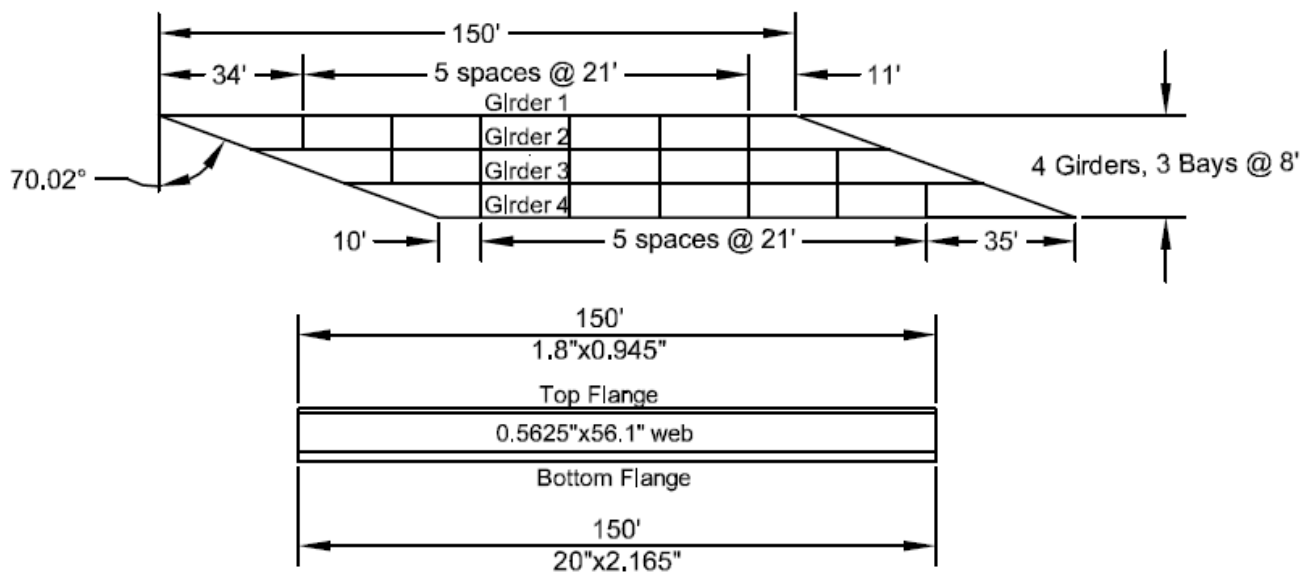

Bridge C

Figure A.3: Framing plans and girder sizes of the Bridge $C$

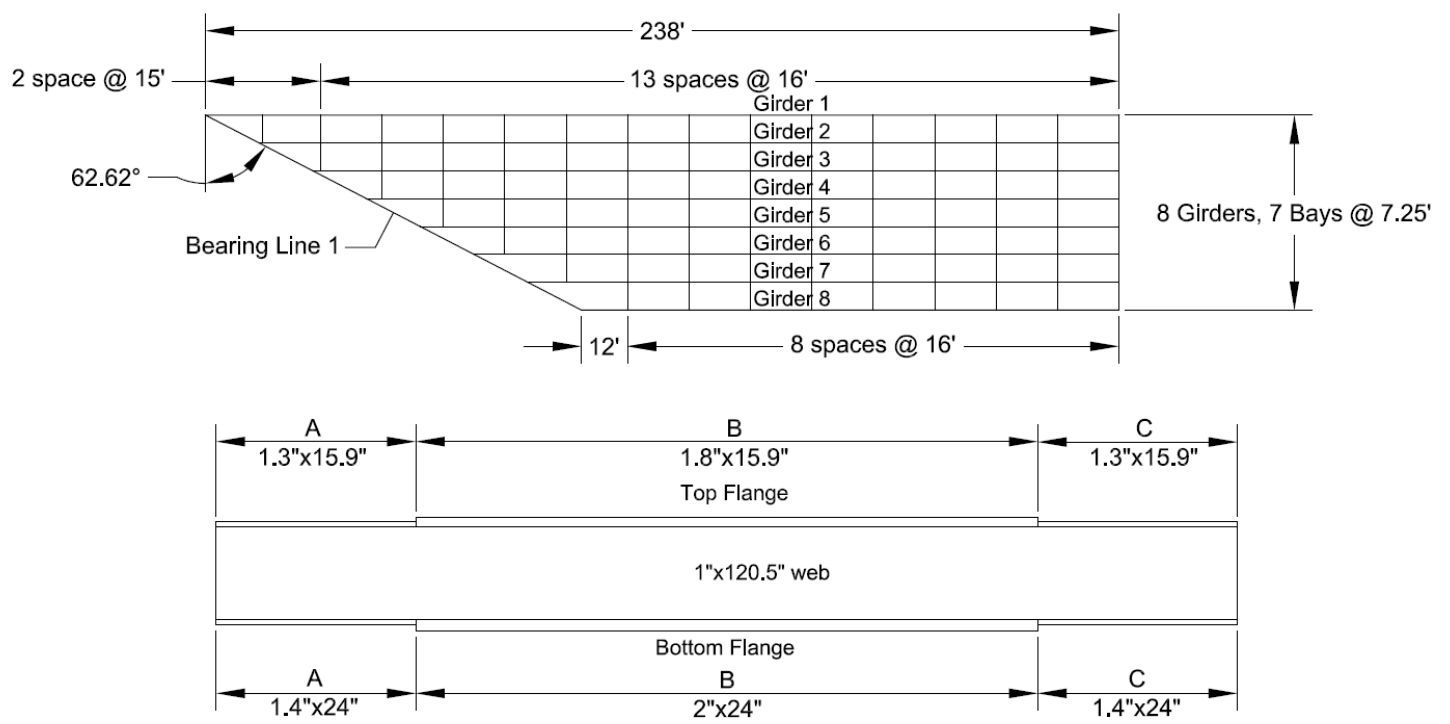

Bridge B2 


\begin{tabular}{|c|c|c|c|c|}
\hline & A (ft.) & B (ft.) & C (ft.) & L (ft.) \\
\hline Girder 1 & 79 & 79 & 80 & 238 \\
\hline Girder 2 & 75 & 75 & 74 & 224 \\
\hline Girder 3 & 70 & 70 & 70 & 210 \\
\hline Girder 4 & 65 & 65 & 66 & 196 \\
\hline Girder 5 & 61 & 61 & 60 & 182 \\
\hline Girder 6 & 56 & 56 & 56 & 168 \\
\hline Girder 7 & 51 & 51 & 52 & 154 \\
\hline Girder 8 & 47 & 47 & 46 & 140 \\
\hline
\end{tabular}

\section{A.2 Loading}

For the steel dead load (SDL) self-weight of girders, stiffeners, and cross-frames is considered. Stiffeners are provided at every cross frame location. The density of steel is taken as 490pcf. The concrete dead load is applied on the top flanges of the girder by considering equivalent width in all the analyses. An overhang equal to half of girder spacing is considered in each bridge providing a uniform line load for all girders. The density of concrete is considered as 150pcf. Thickness of deck is taken as 8inch in analysis of all the bridges. No lateral load from wind or knee brace is applied in order to distinguish the component of structural responses from the lack-of-fit. 


\section{APPENDIX B COMPARISON OF DETAILING METHODS FOR BRIDGE}

\section{B AND BRIDGE C}

\section{B.1 Concrete Dead Load Deflections}

B.1.1 Bridge A
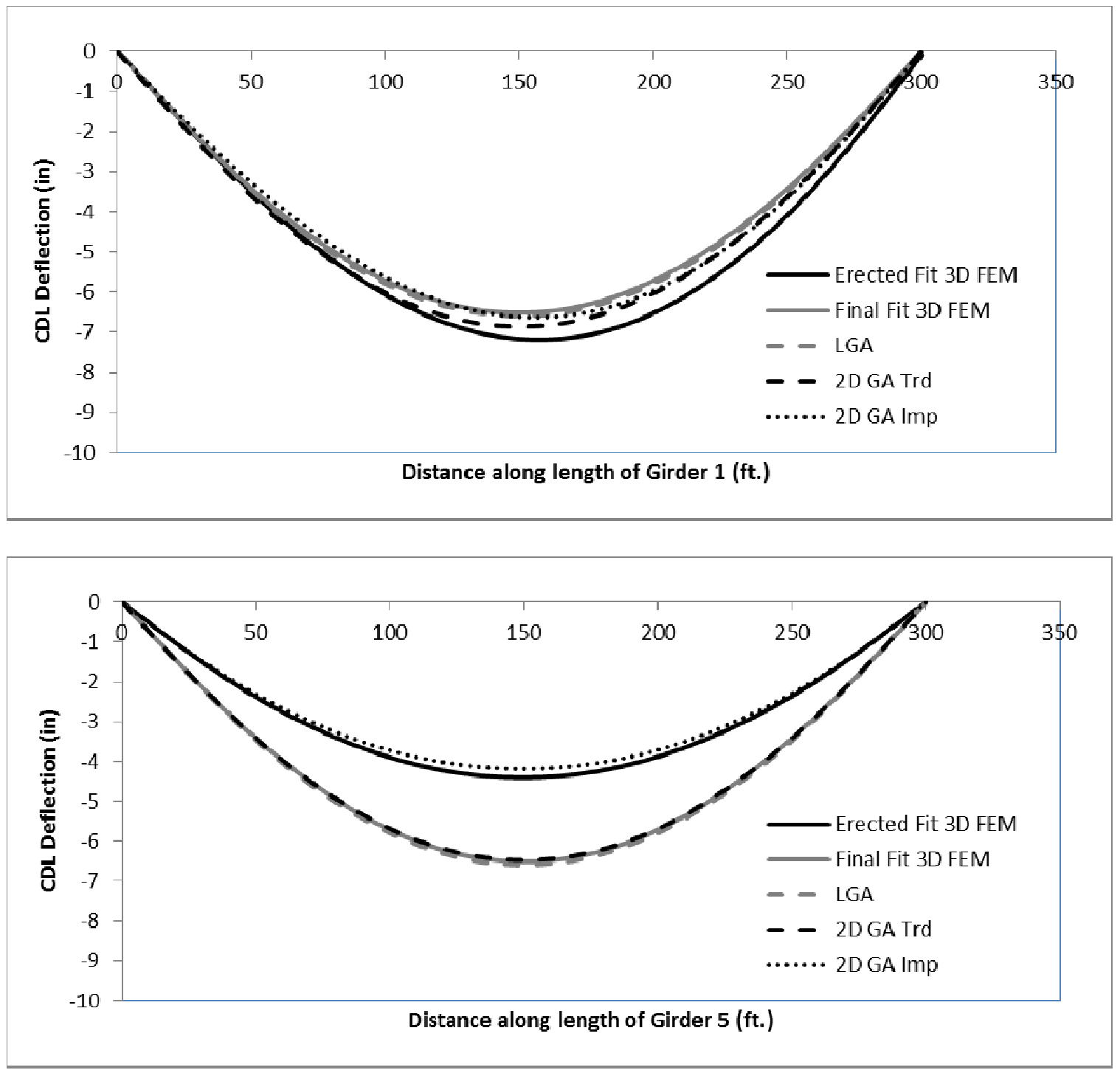


\section{B.1.2 Bridge $B$}

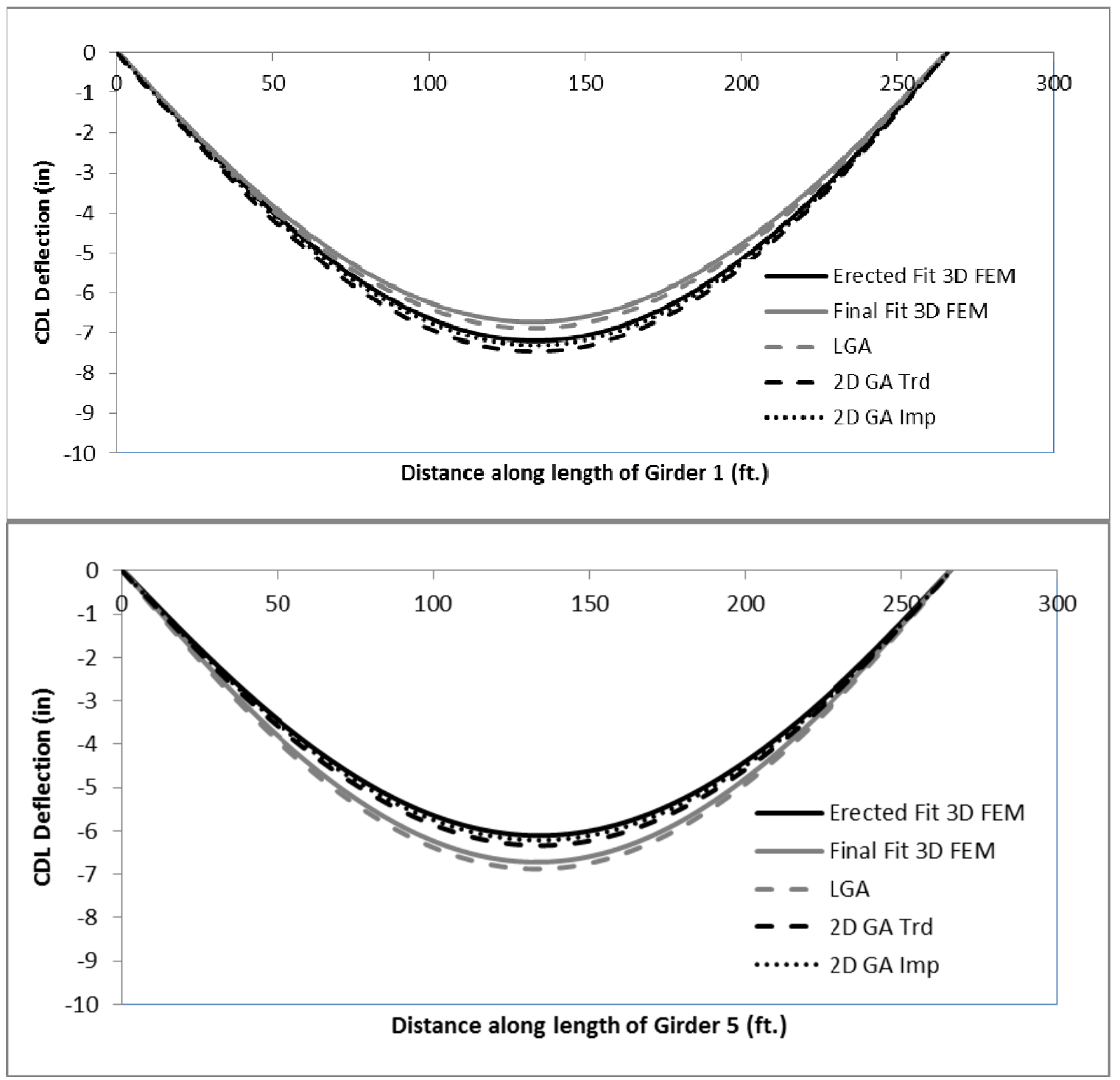




\section{B.1.3 Bridge C}

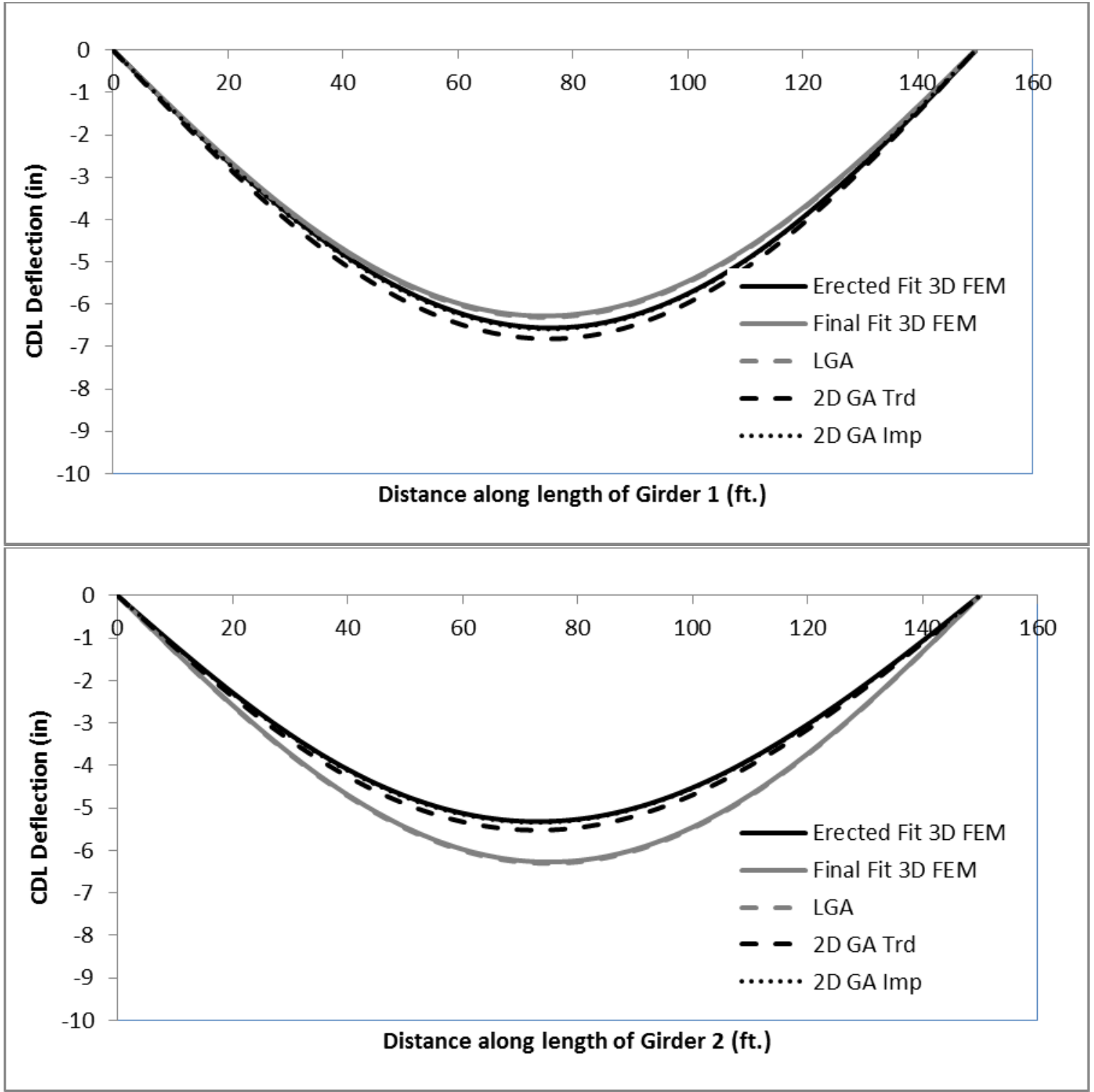




\section{B.1.4 Bridge B2}
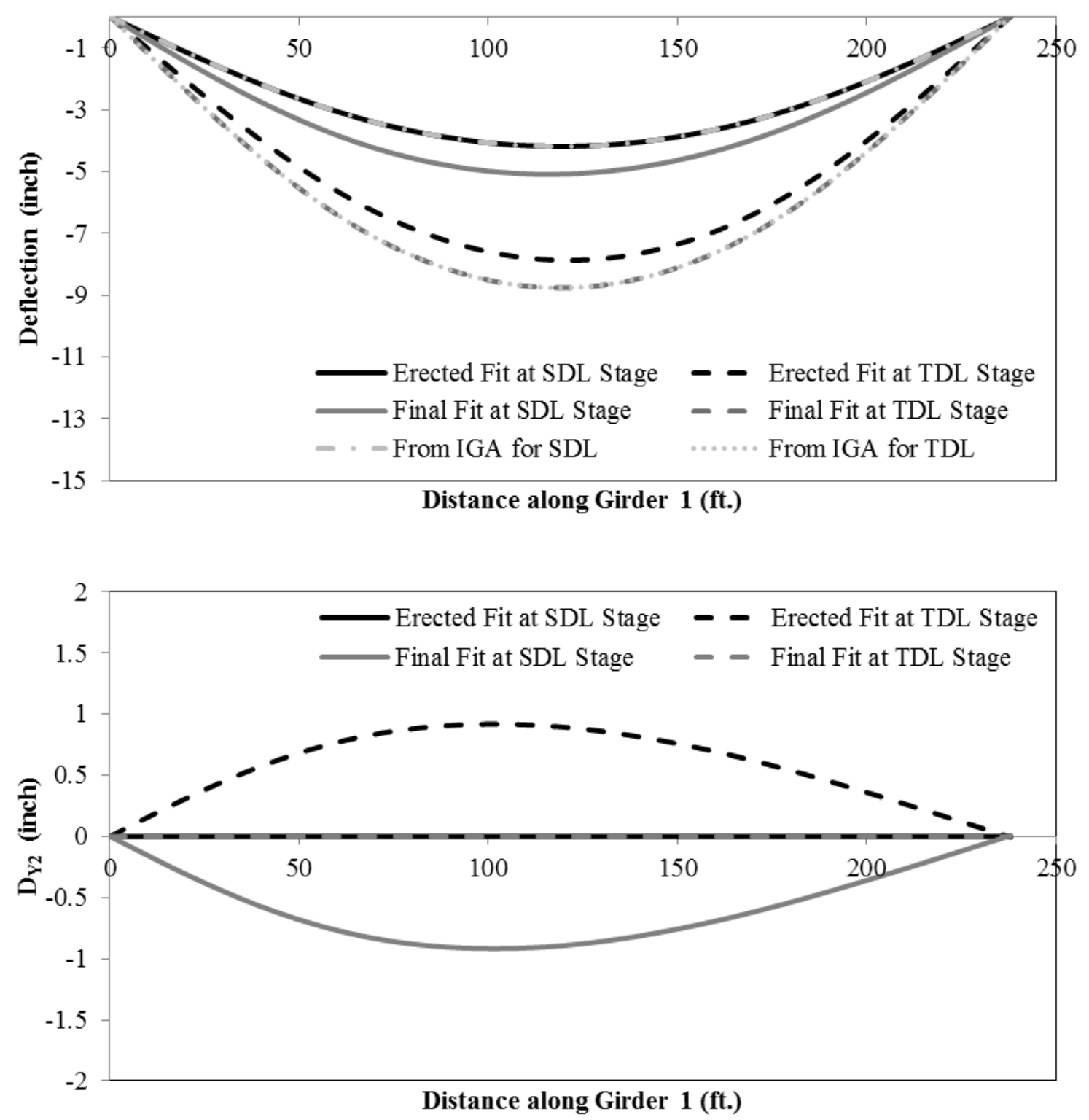


\section{B.2 Layovers}

\section{B.2.1 Bridge A}
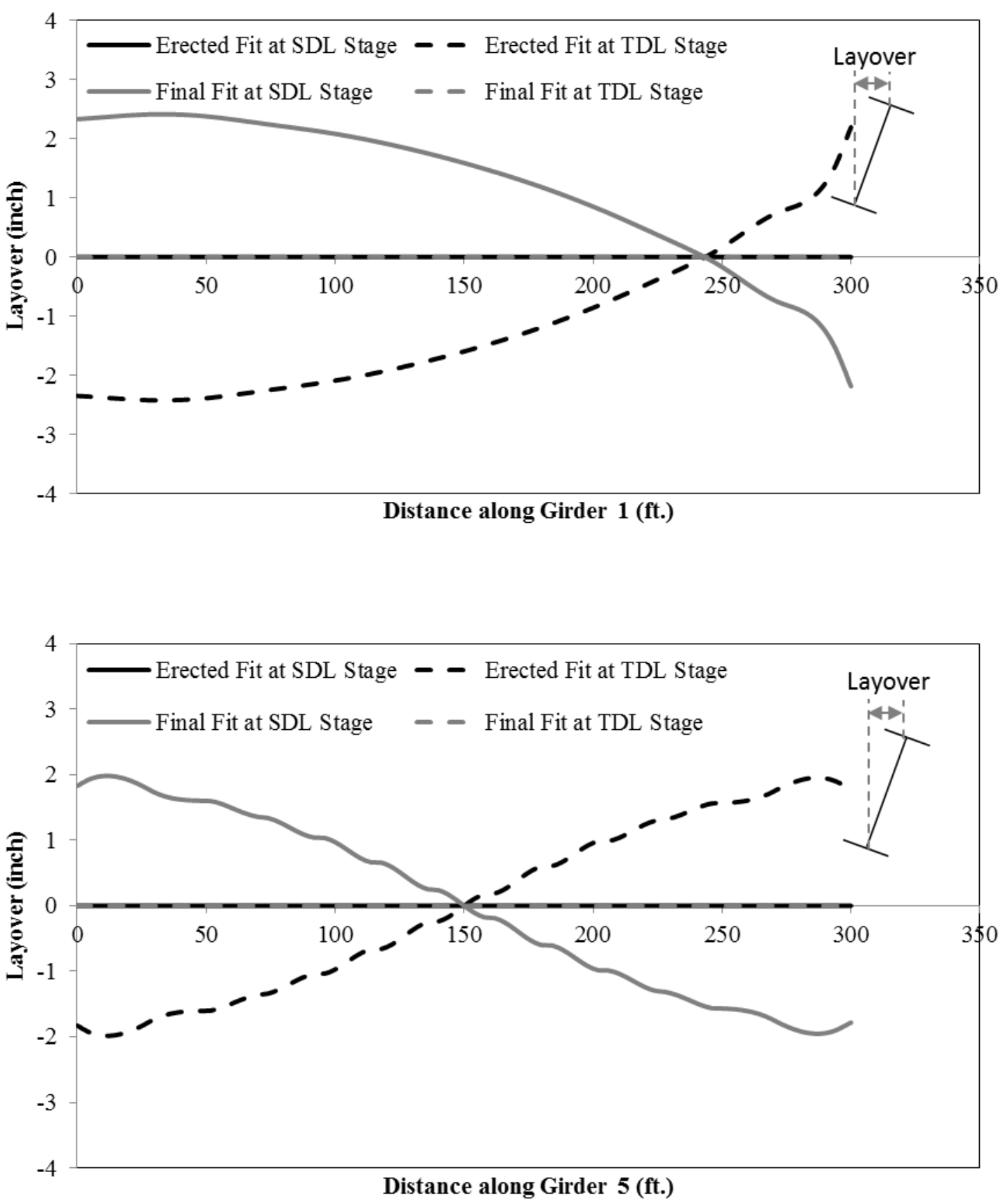


\section{B.2.2 Bridge B}
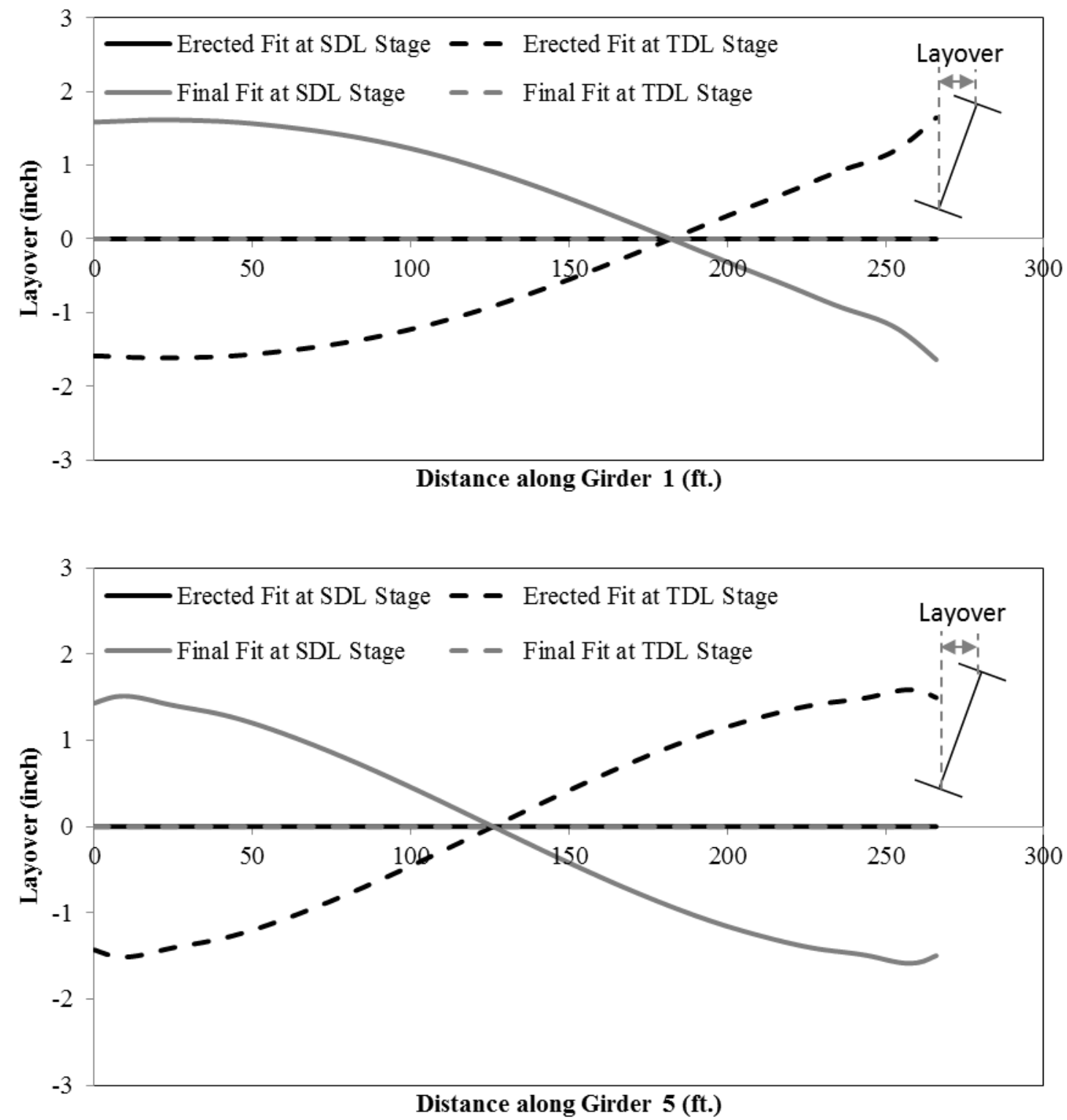


\section{B.2.3 Bridge C}
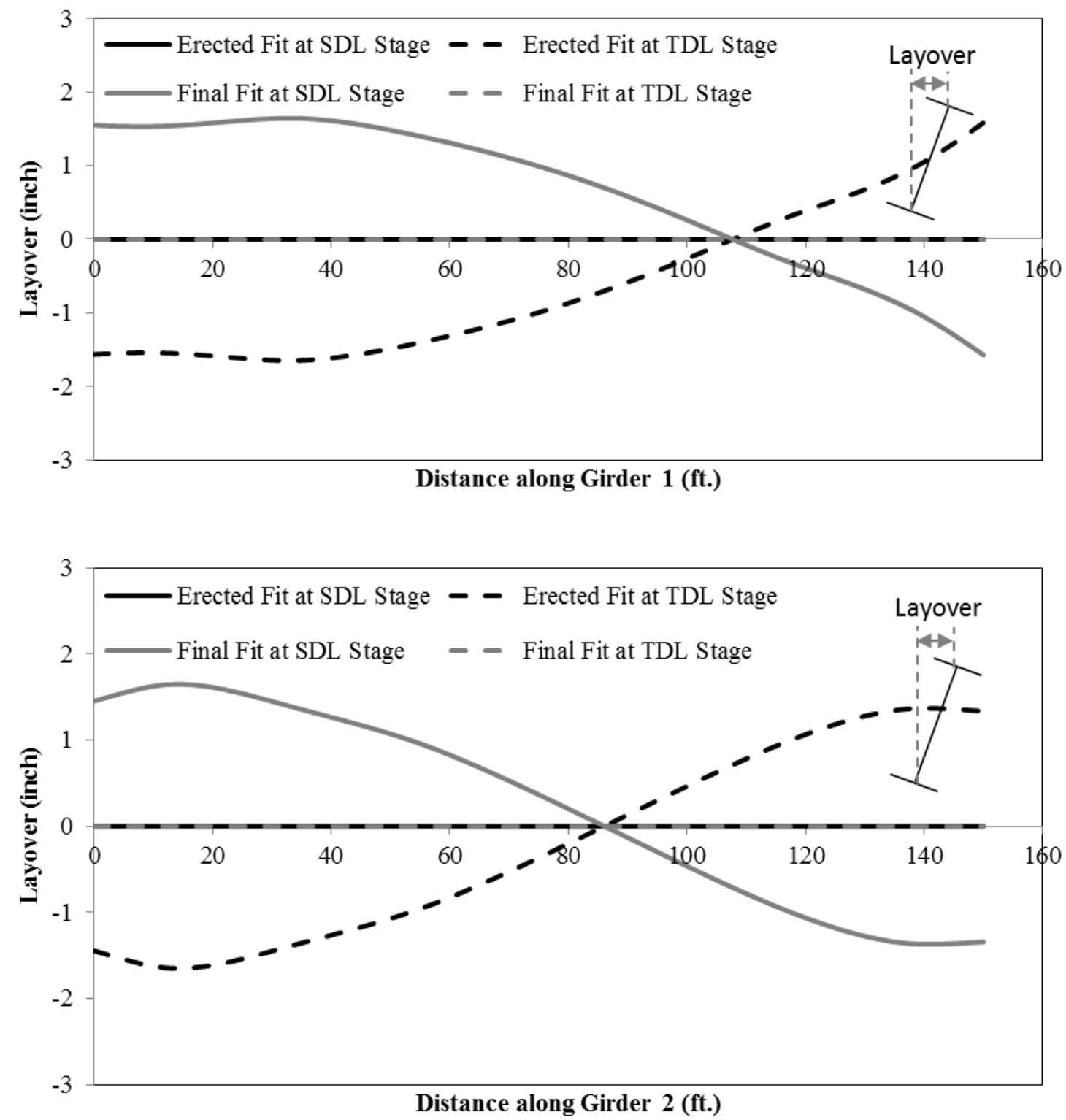


\section{B.2.4 Bridge B2}

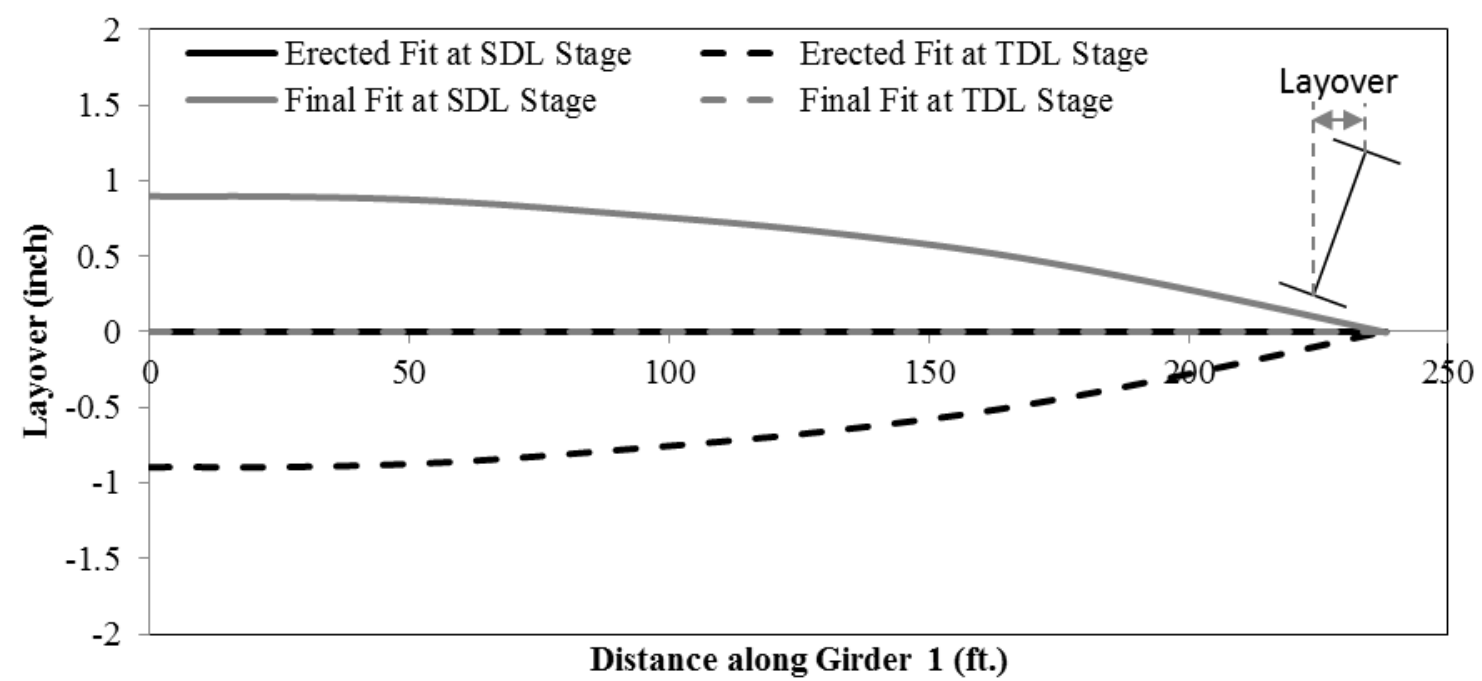




\section{B.3 Flange Lateral Bending Stress}

\section{B.3.1 Bridge A}
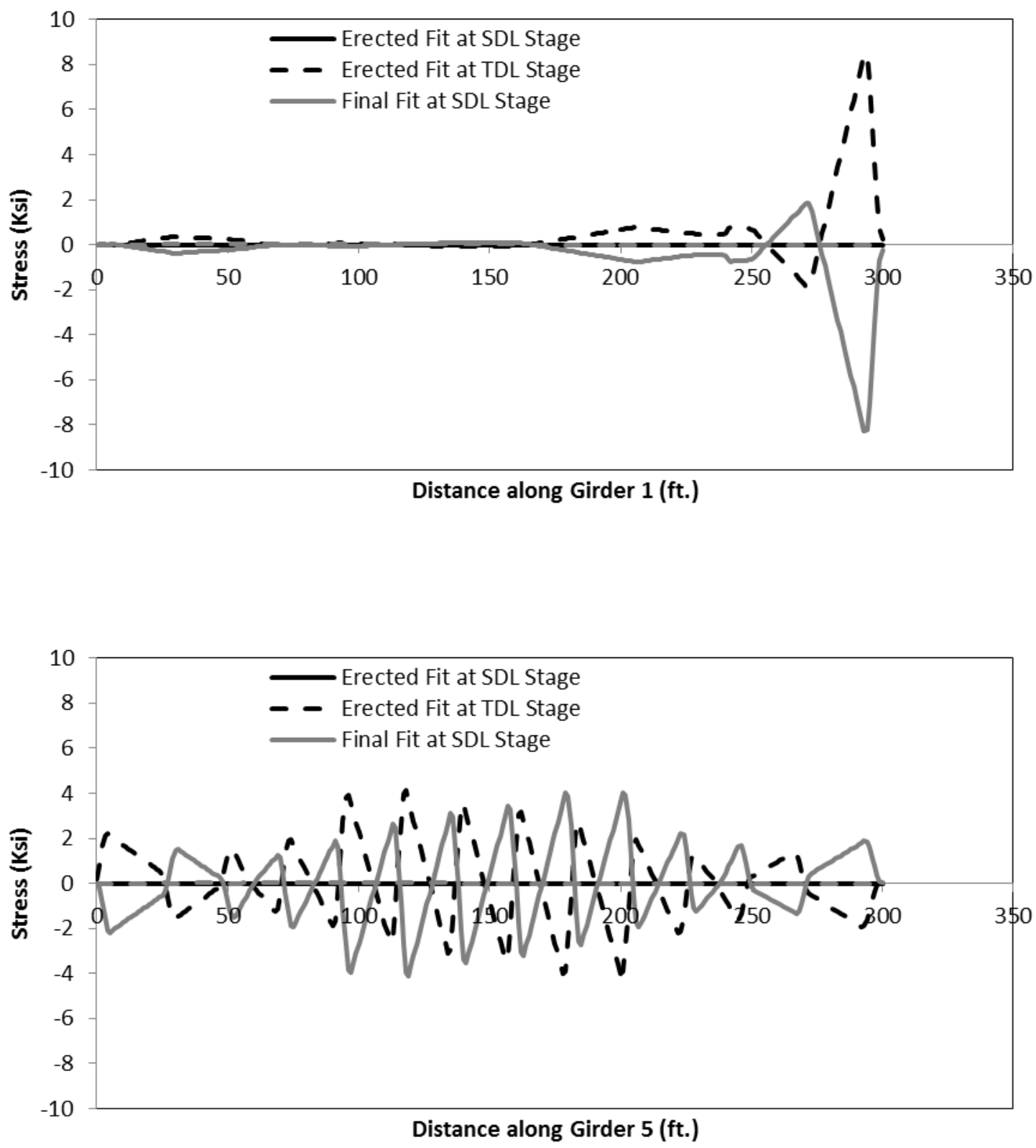


\section{B.3.2 Bridge B}
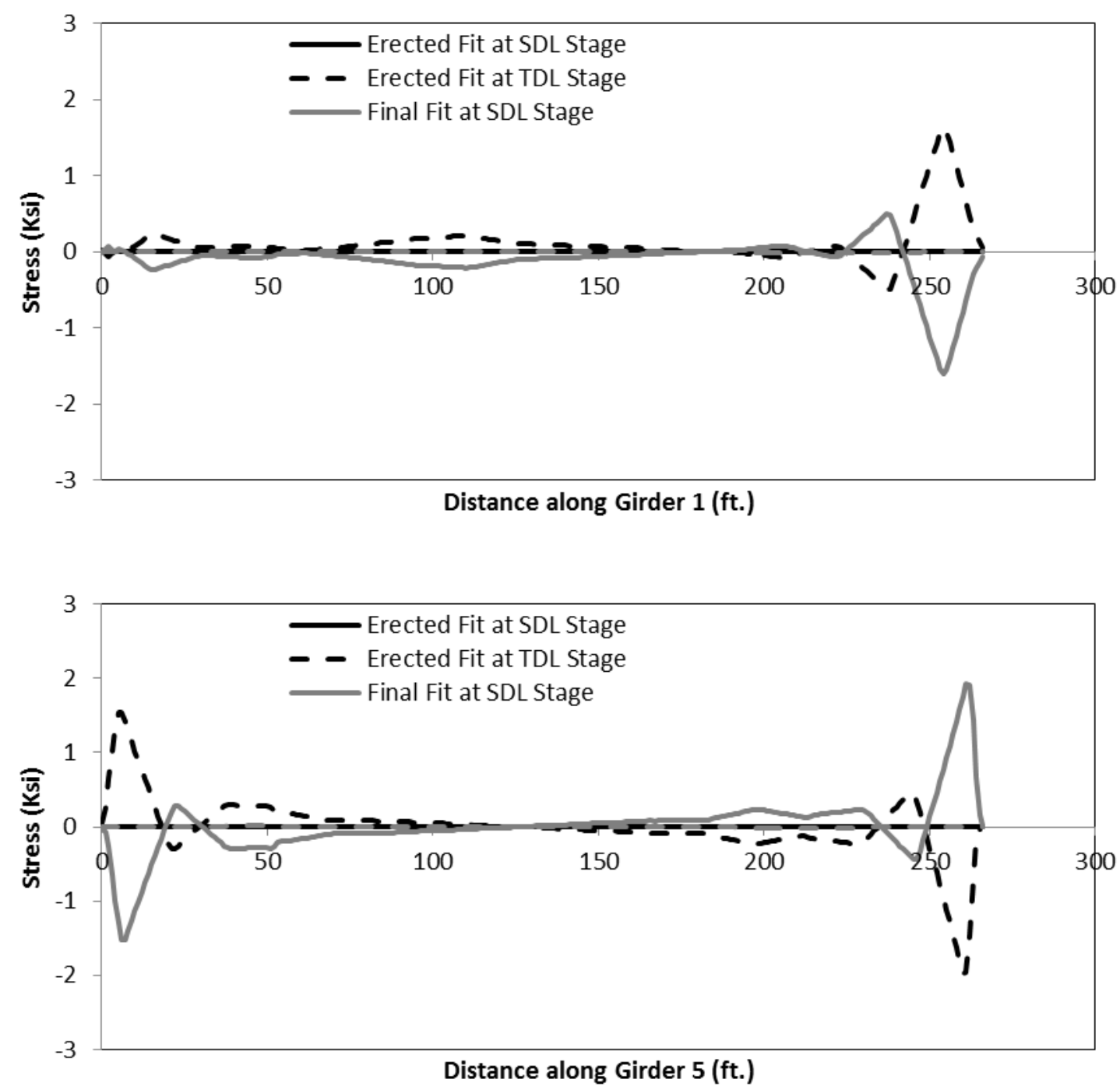


\section{B.3.3 Bridge C}
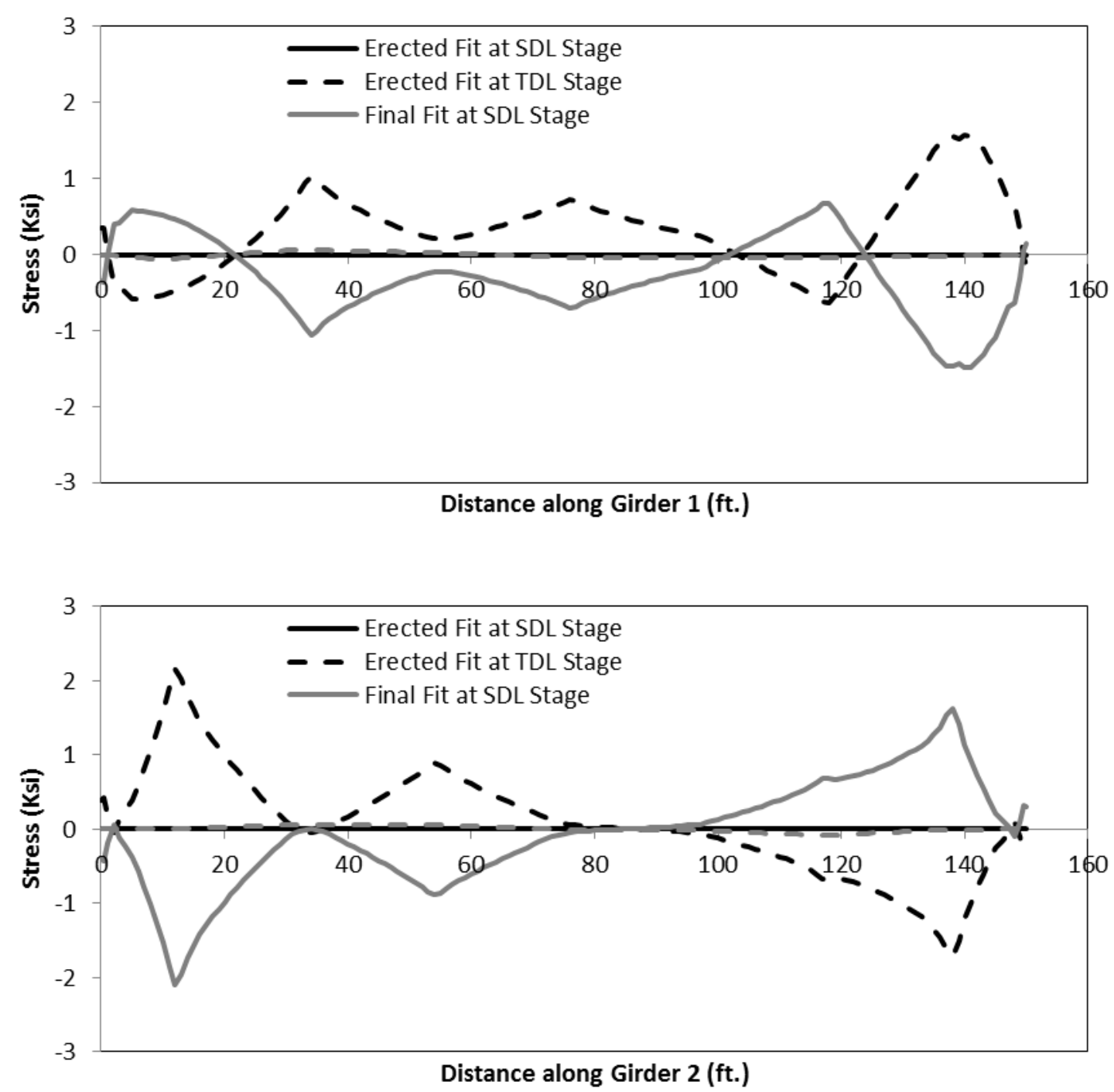


\section{B.3.4 Bridge B2}

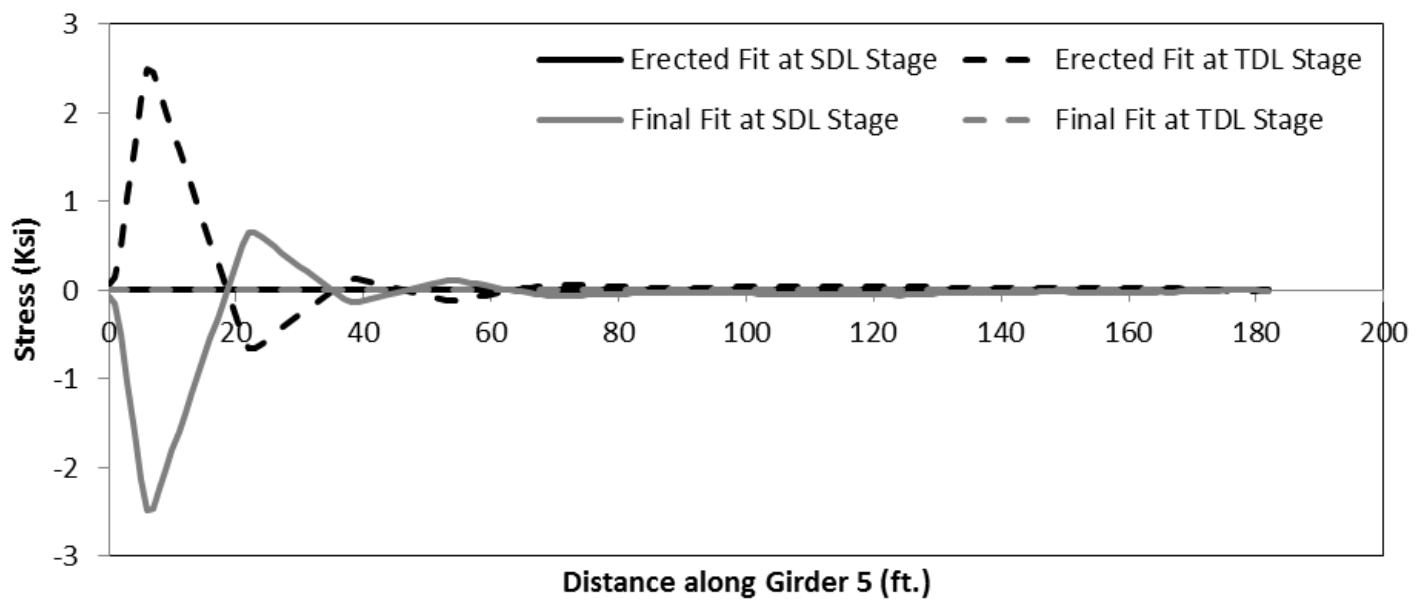




\section{B.4 Reactions}

\section{B.4.1 Bridge A}

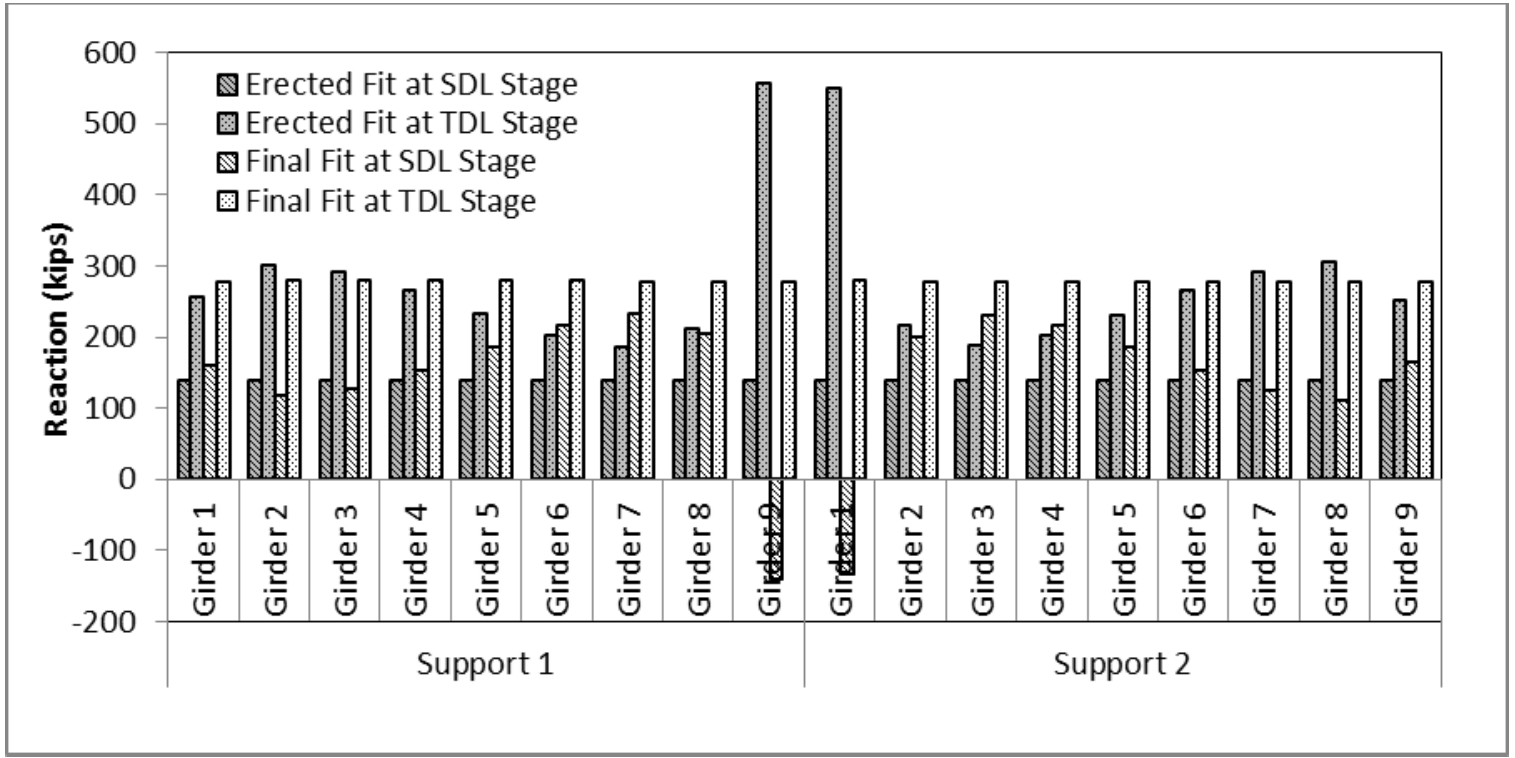

\section{B.4.2 Bridge B}

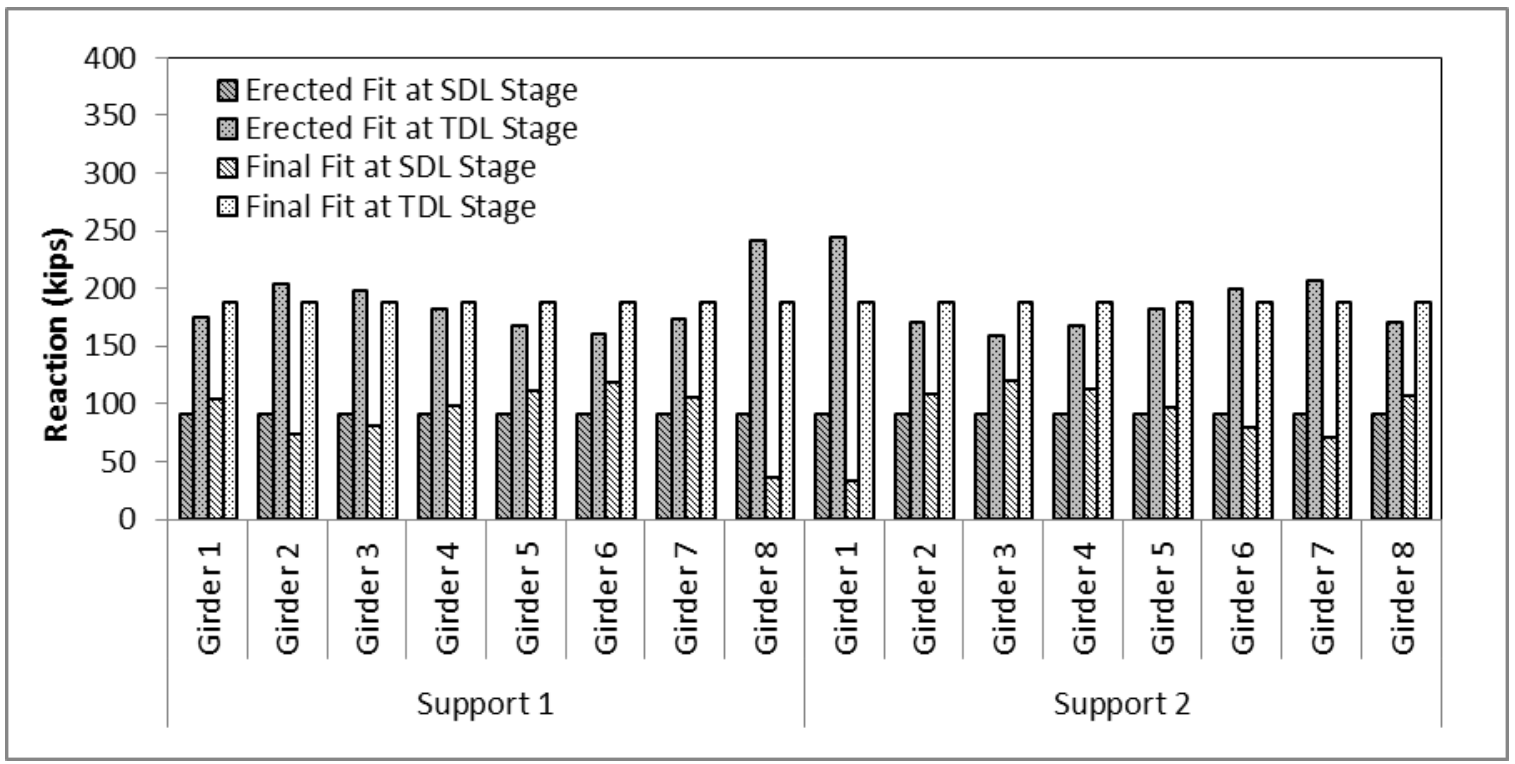




\section{B.4.3 Bridge $C$}

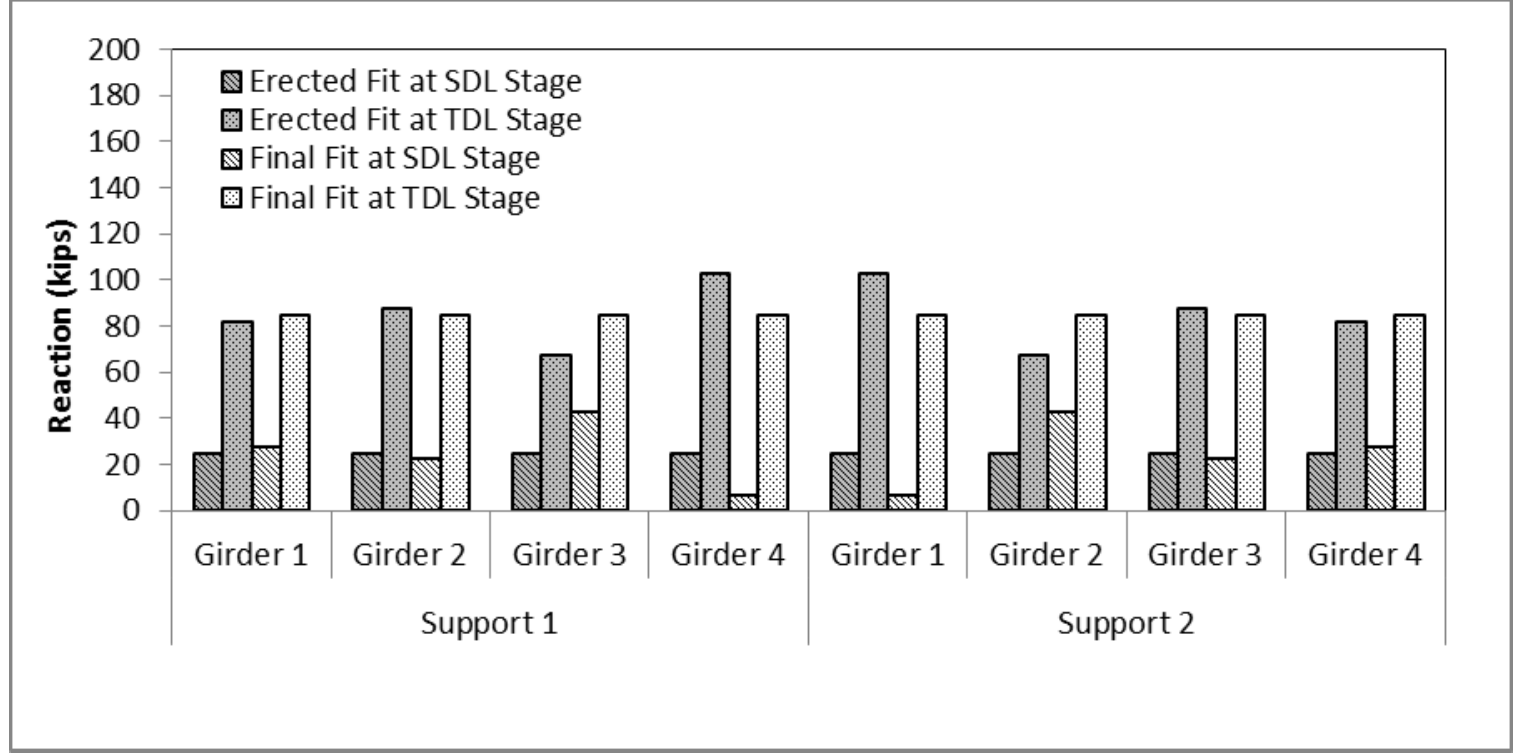

\section{B.4.4 Bridge B2}

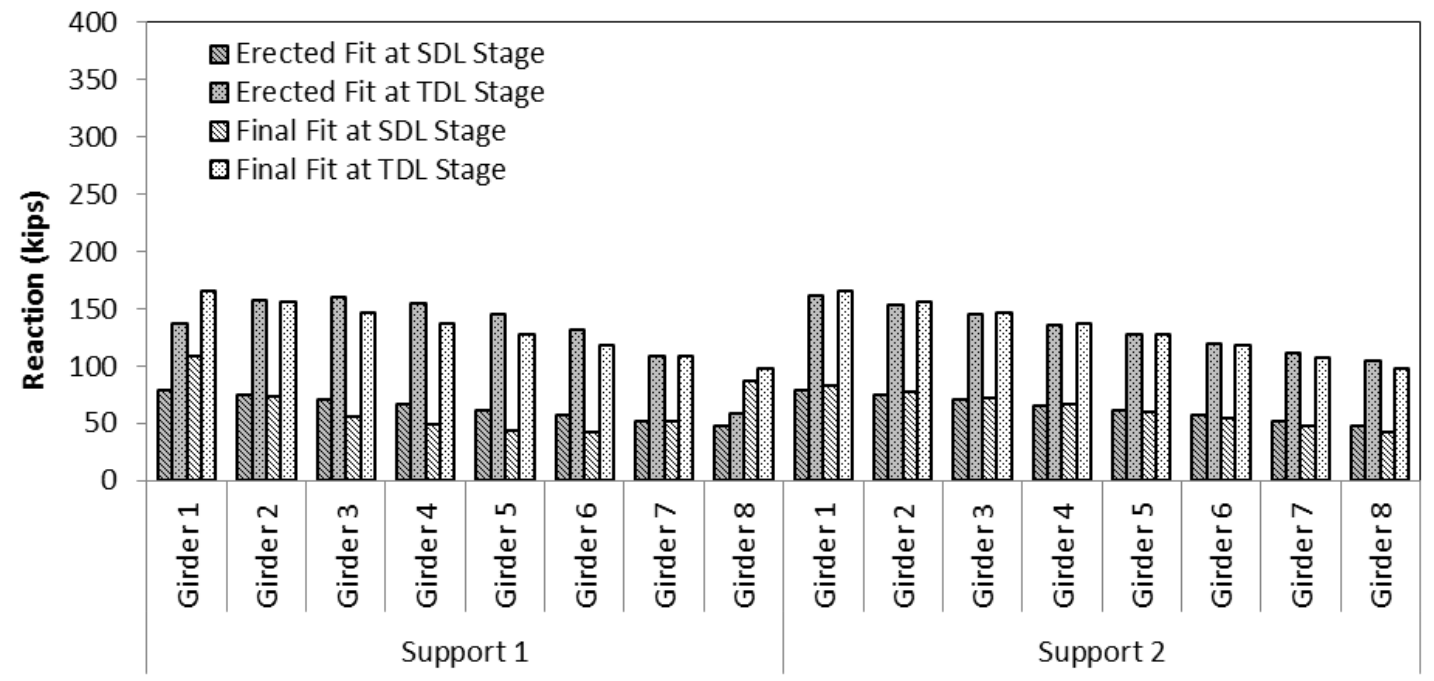




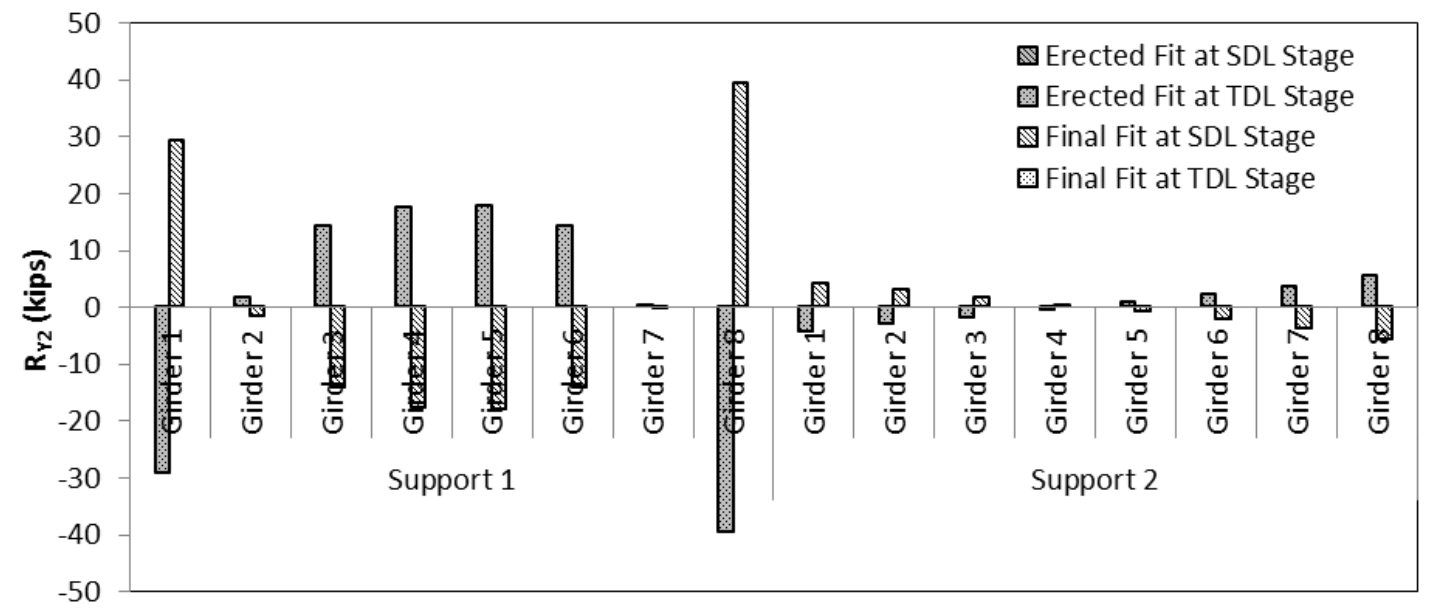




\section{B.5 Cross Frame Forces}

\section{B.5.1 Bridge A}
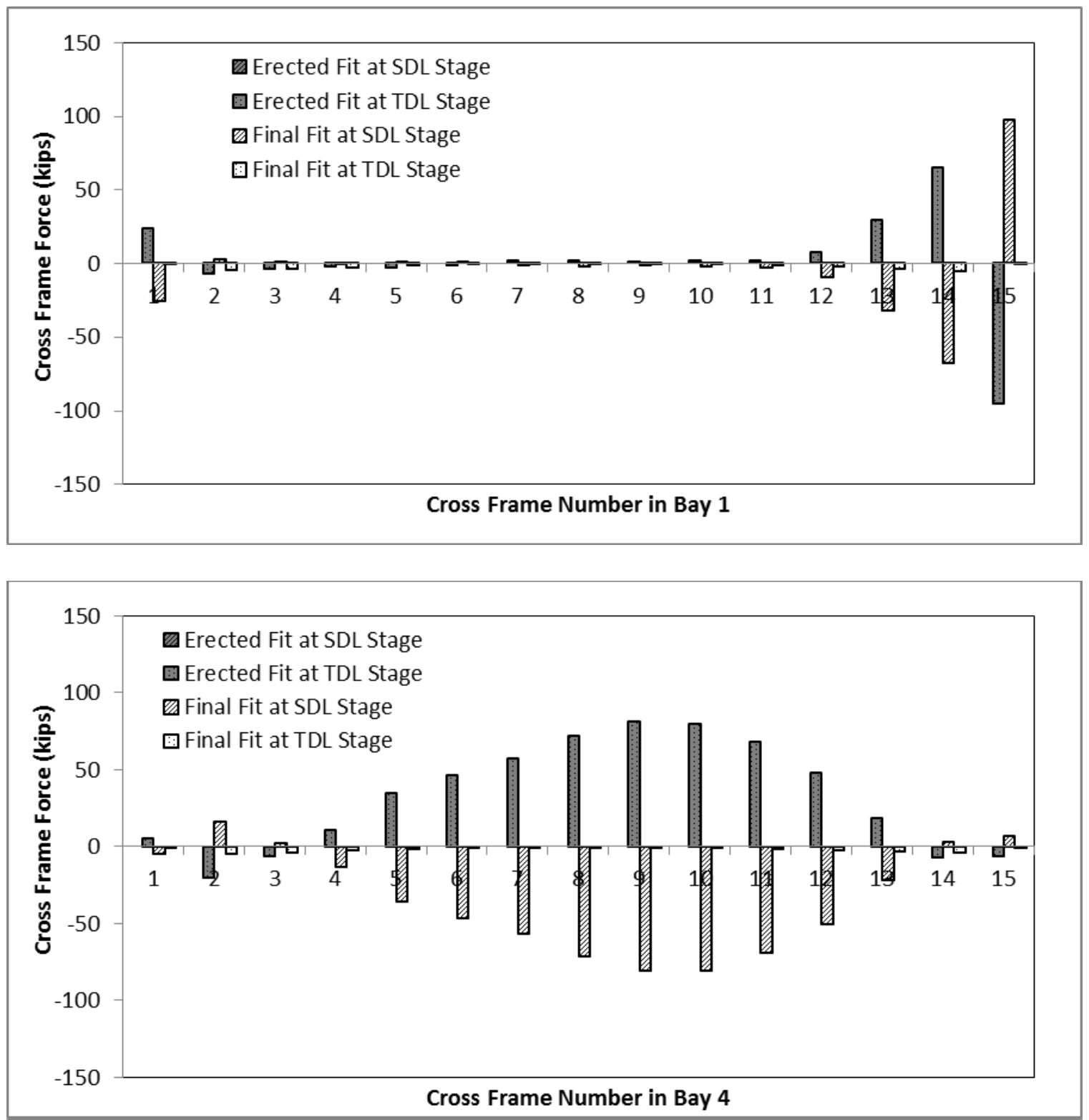


\section{B.5.2 Bridge $B$}

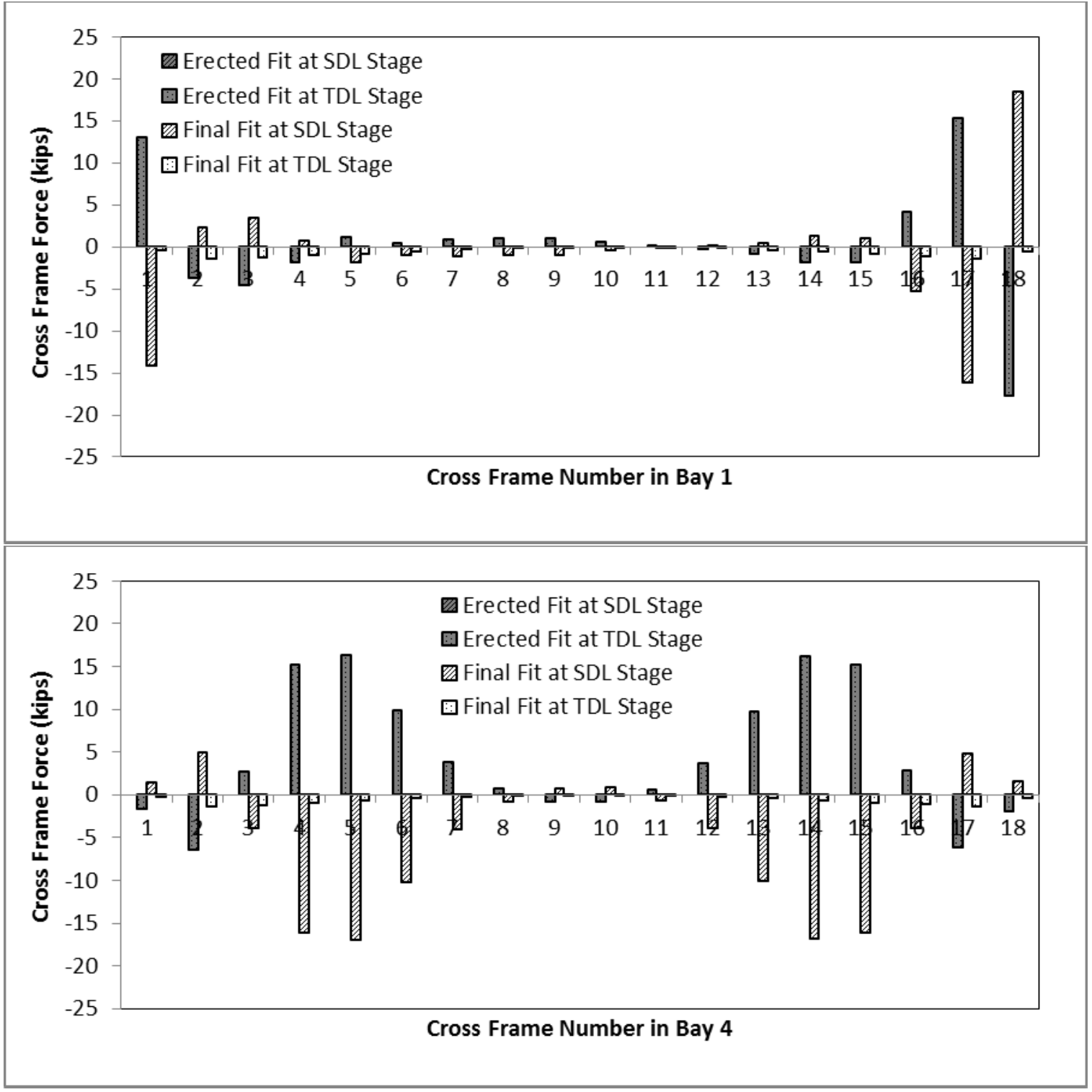




\section{B.5.3 Bridge $C$}
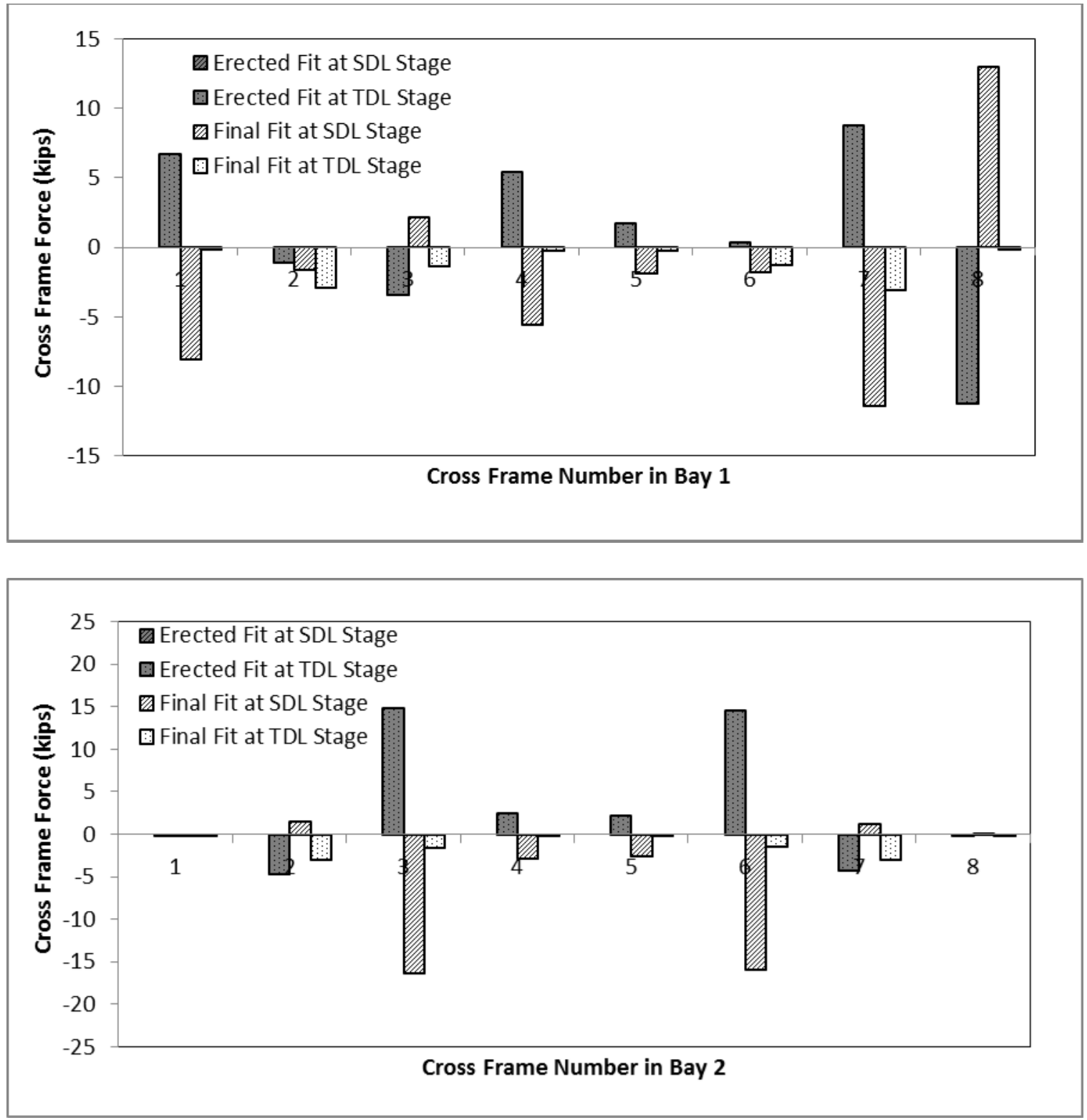


\section{B.5.4 Bridge B2}

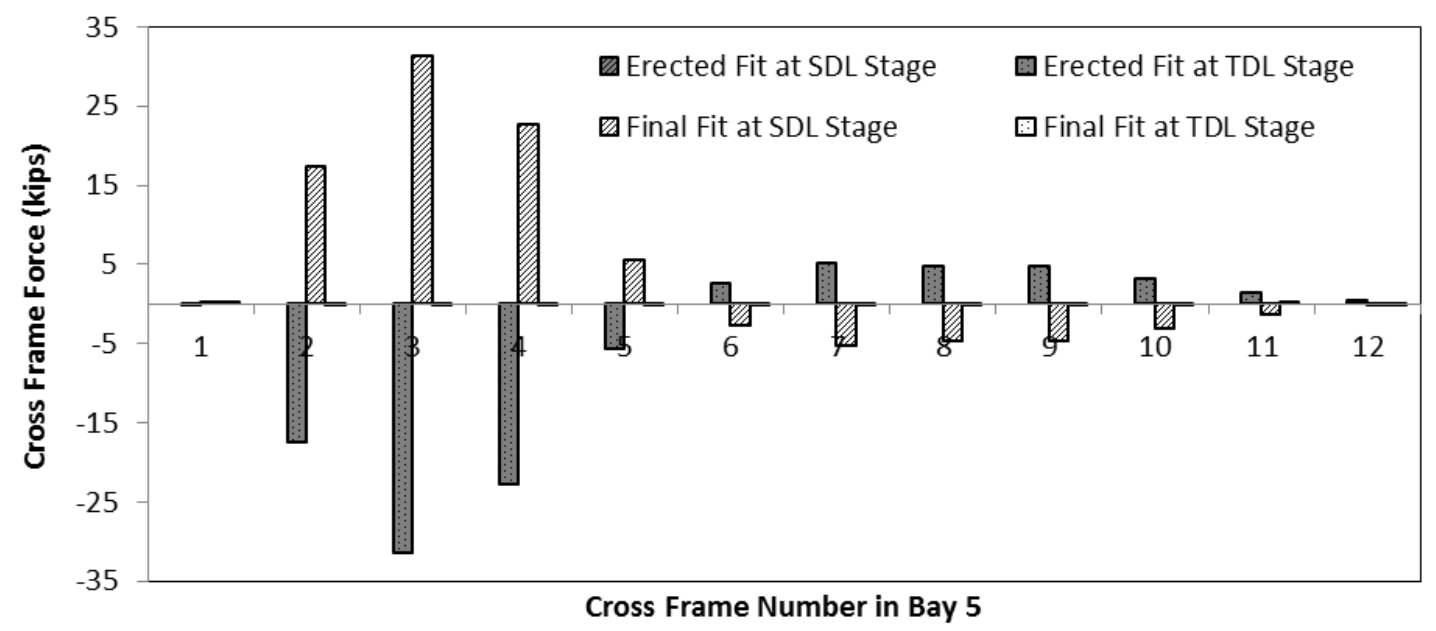




\section{APPENDIX C METHODS OF ANALYSIS-ERECTED FIT}

\section{C.1 Girder Stiffness Matrix}

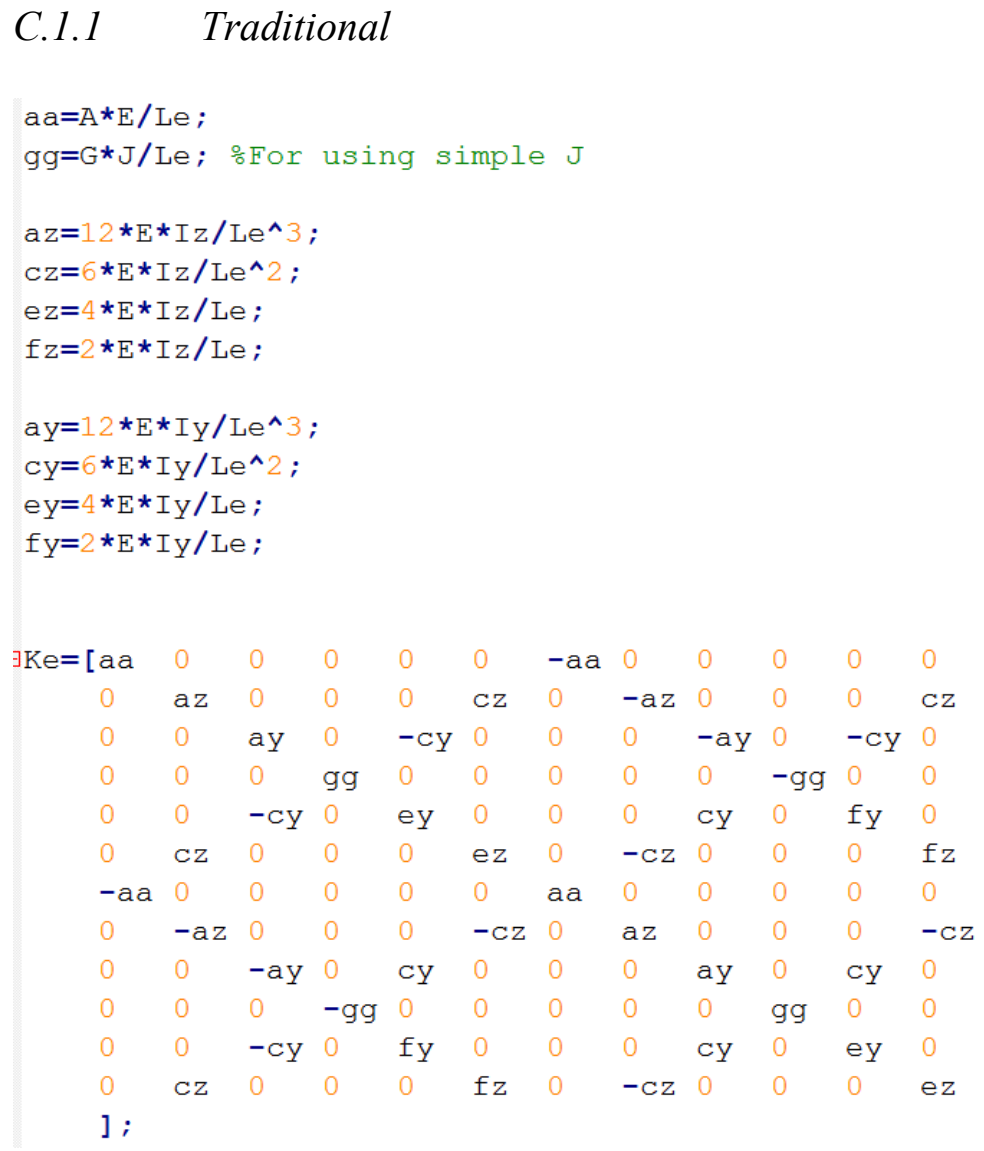

Le is the length of the element, Iy is moment of inertia about minor axis, Iz is moment of inertia about major axis, and $\mathrm{E}$ is modulus of elasticity of steel. 
This matrix uses Jeq calculated as follows:

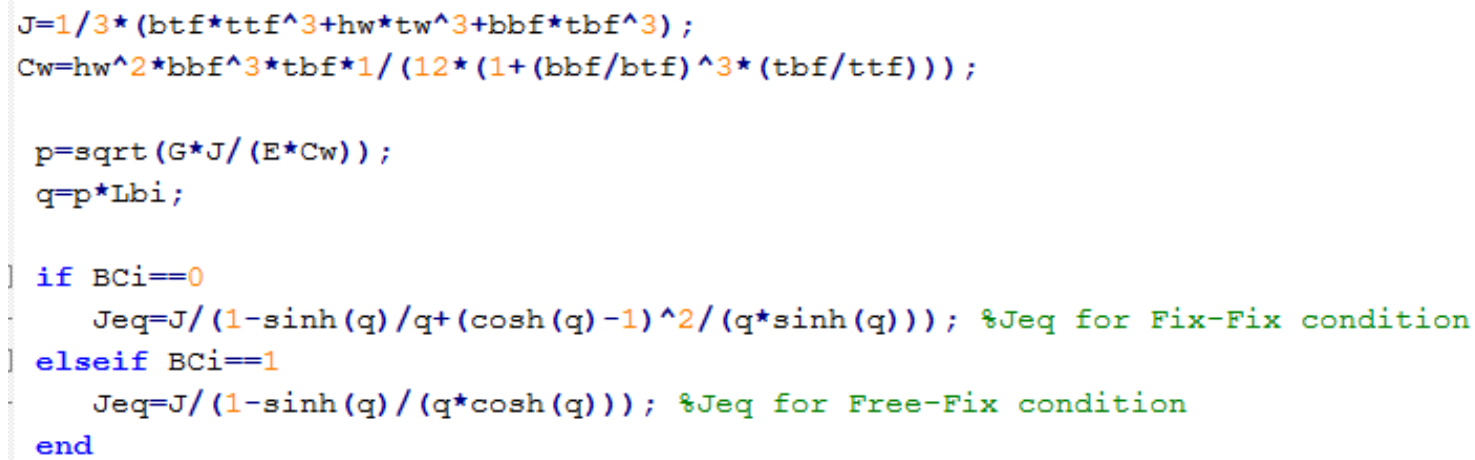

Lbi is the unbrace length of the particular element. All the elements between two consecutive cross have same un-braced length equal to distance between the two consecutive cross frames. Rest of the calculations is same as in traditional matrix for girders. 


\section{C.2 Cross-frame Stiffness Matrix}

\section{C.2.1 Traditional}

The following matrix is for X-type cross-frame.

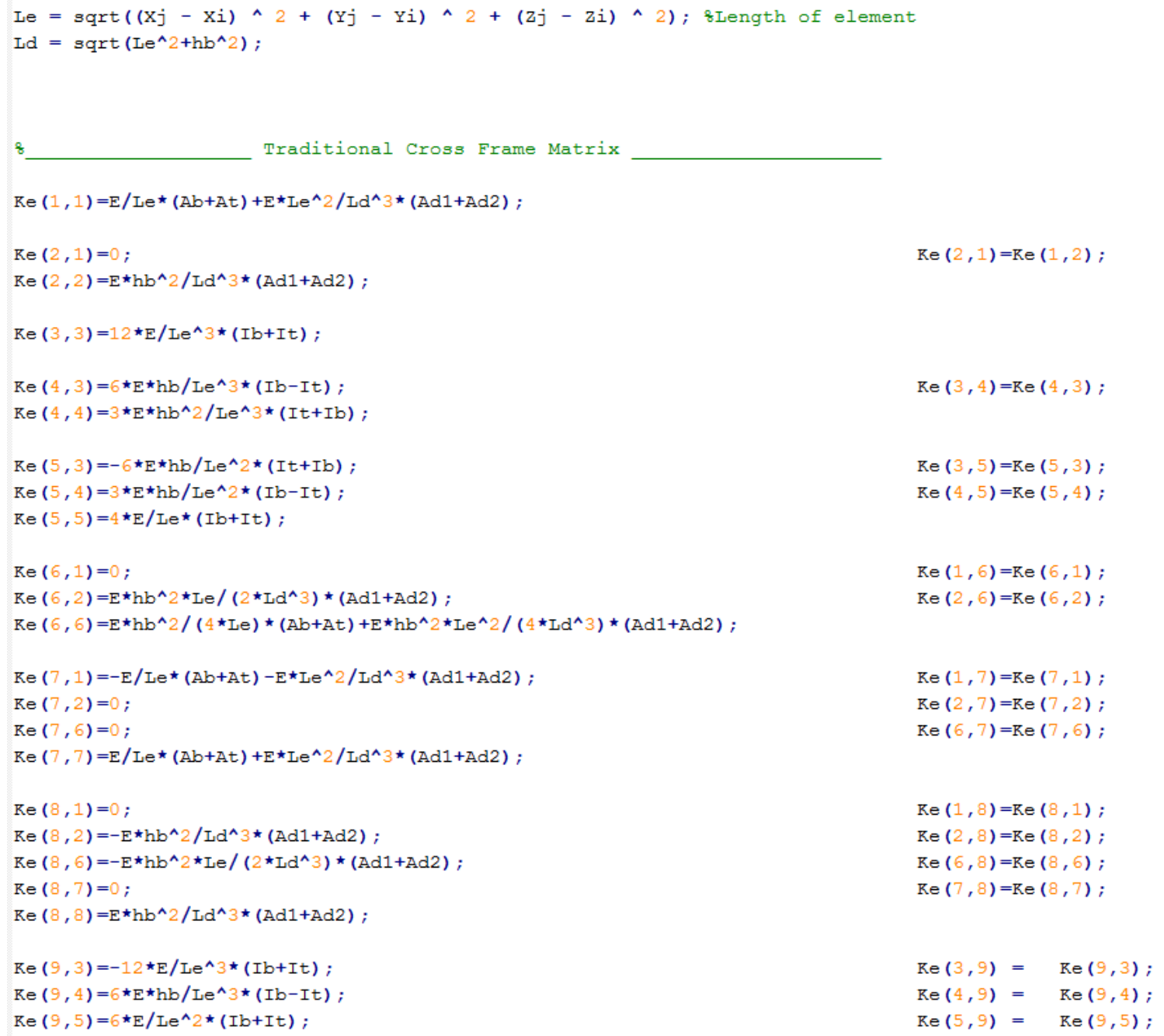


$\operatorname{Ke}(10,3)=6 \star \mathrm{E}^{\star} \mathrm{hb} / \mathrm{Le} e^{\wedge} 3(\mathrm{Ib}-\mathrm{It}) ;$

$\operatorname{Ke}(10,4)=-3 \star \mathrm{E}^{\star} \mathrm{hb} \mathrm{b}^{\wedge} 2 / \mathrm{Le} \mathrm{e}^{\wedge} 3{ }^{\star}(\mathrm{It}+\mathrm{Ib}) ;$

$\operatorname{Ke}(10,5)=3 \star E^{\star} \mathrm{hb} / \mathrm{Le}^{\wedge} 2 \star($ It $-\mathrm{Ib}) ;$

$\operatorname{Ke}(10,9)=6{ }^{\star} \mathrm{Hb}^{\star} \mathrm{hb} \mathrm{Le}^{\wedge \star}(\mathrm{It}-\mathrm{Ib}) ;$

$\operatorname{Re}(10,10)=3 \star E^{\star} h b^{\wedge} 2 / L e^{\wedge} 3 \star(I b+I t) ;$

$\operatorname{Re}(11,3)=-6 \star E / L e^{\wedge} 2 \star(I t+I b) ;$

$\operatorname{Ke}(11,4)=3 \star E^{\star} \mathrm{hb} / \mathrm{Le}^{\wedge} 2 \star(\mathrm{Ib}-\mathrm{It})$;

$\operatorname{Ke}(11,5)=2 \star E / L e^{\star}(I b+I t) ;$

$\operatorname{Ke}(11,9)=6 \star E / L e^{\wedge} 2 \star(I t+I b)$;

$\operatorname{Ke}(11,10)=3 \star \mathrm{E}^{\star} \mathrm{hb} / \mathrm{Le} \mathrm{e}^{\wedge} 2$ (It-Ib) ;

$\operatorname{Re}(11,11)=2 \star \mathrm{E} / \mathrm{Le}{ }^{\star}(\mathrm{It}+\mathrm{Ib})$;

$\operatorname{Ke}(12,1)=0$;

$\operatorname{Ke}(12,2)=\mathrm{E}^{\star} \mathrm{hb} \mathrm{b}^{\wedge} 2 \star \mathrm{Le} /\left(2 \star \mathrm{Ld}^{\wedge} 3\right) \star(\operatorname{Ad} 1+\mathrm{Ad} 2) ;$

$\operatorname{Ke}(12,6)=-E^{\star} h b^{\wedge} 2 /(4 \star L e) \star(A b+A t)+E^{\star} h b^{\wedge} 2 \star L e^{\wedge} 2 /\left(4 \star L_{d}^{\wedge} 3\right) \star(A d 1+A d 2) ;$

$\operatorname{Ke}(12,7)=0$;

$\operatorname{Ke}(12,8)=-\mathrm{E}^{\star} \mathrm{hb}^{\wedge} 2 \star \operatorname{Le} /\left(2 \star \mathrm{Ld}^{\wedge} 3\right) \star(\operatorname{Ad} 1+\mathrm{Ad} 2) ;$

$\operatorname{Ke}(12,12)=\mathrm{E}^{\star} \mathrm{hb}^{\wedge} 2 /(4 \star \mathrm{Le}) \star(\mathrm{Ab}+\mathrm{At})+\mathrm{E}^{\star} \mathrm{h} \mathrm{b}^{\wedge} 2 \star \mathrm{Le} \mathrm{e}^{\wedge} 2 /\left(4 \star \mathrm{Ld}^{\wedge} 3\right) \star(\operatorname{Ad} 1+\mathrm{Ad} 2)$;

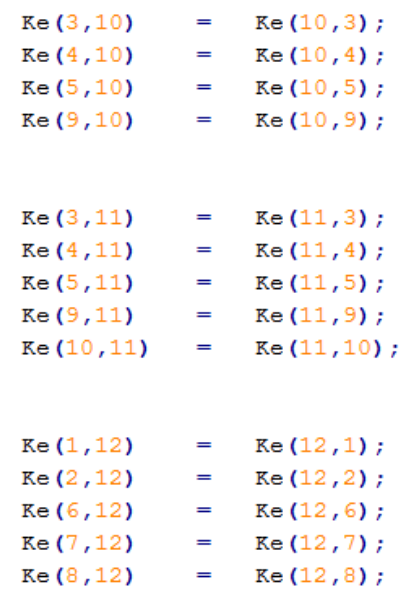

Le is length of cross frame element equal to spacing between the girders, $\mathrm{Ld}$ is length of diagonal members in $\mathrm{X}$-type cross frame, $\mathrm{Ab}$ is cross section area of bottom chord, At is cross section area of top chord, Ad1 is cross section area of diagonal 1 and $\mathrm{Ad} 2$ is cross section area of diagonal 2 , hb is height of bracing or cross frame, $\mathrm{Ib}$ is moment of inertia of bottom chord about an axis parallel to height of cross-frame, It is moment of inertia of top chord about an axis parallel to height of cross-frame.

\section{C.2.2 Improved}

Add following to the traditional matrix

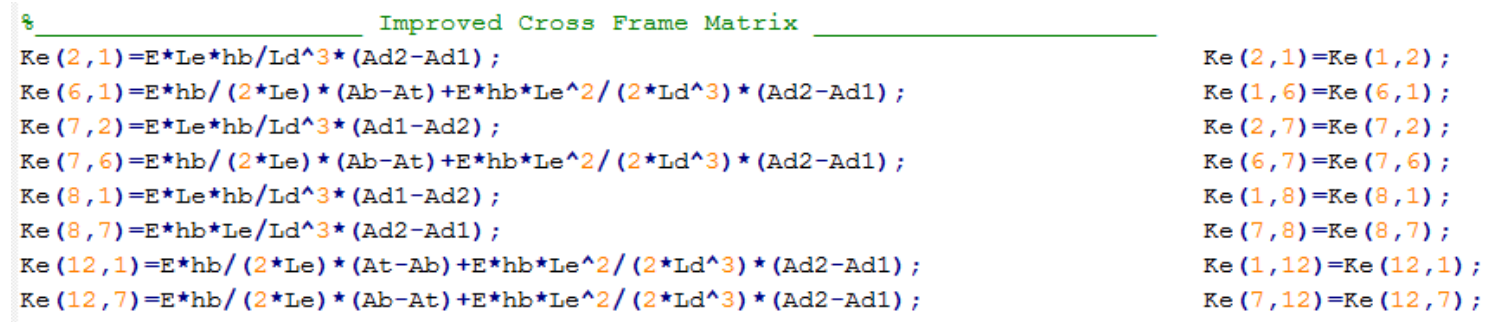

$\operatorname{Ke}(2,1)=\operatorname{Ke}(1,2) ;$

$\operatorname{Ke}(1,6)=\operatorname{Ke}(6,1)$;

$\operatorname{Ke}(2,7)=\operatorname{Ke}(7,2)$;

$\operatorname{Ke}(6,7)=\operatorname{Ke}(7,6) ;$

$\operatorname{Ke}(1,8)=\operatorname{Ke}(8,1)$;

$\operatorname{Ke}(7,8)=\operatorname{Ke}(8,7)$;

$\operatorname{Ke}(1,12)=\operatorname{Ke}(12,1)$;

$\operatorname{Ke}(7,12)=\operatorname{Ke}(12,7)$; 


\section{C.3 Comparison of results using improved and traditional cross frame matrix}

In order to evaluate improvement made by improved cross frame matrix, flange lateral bending stress and cross frames forces are compared. It has been found that both responses have almost the same value for both improved and traditional cross frame matrix. The improved cross frame matrix do not significantly improved the result.

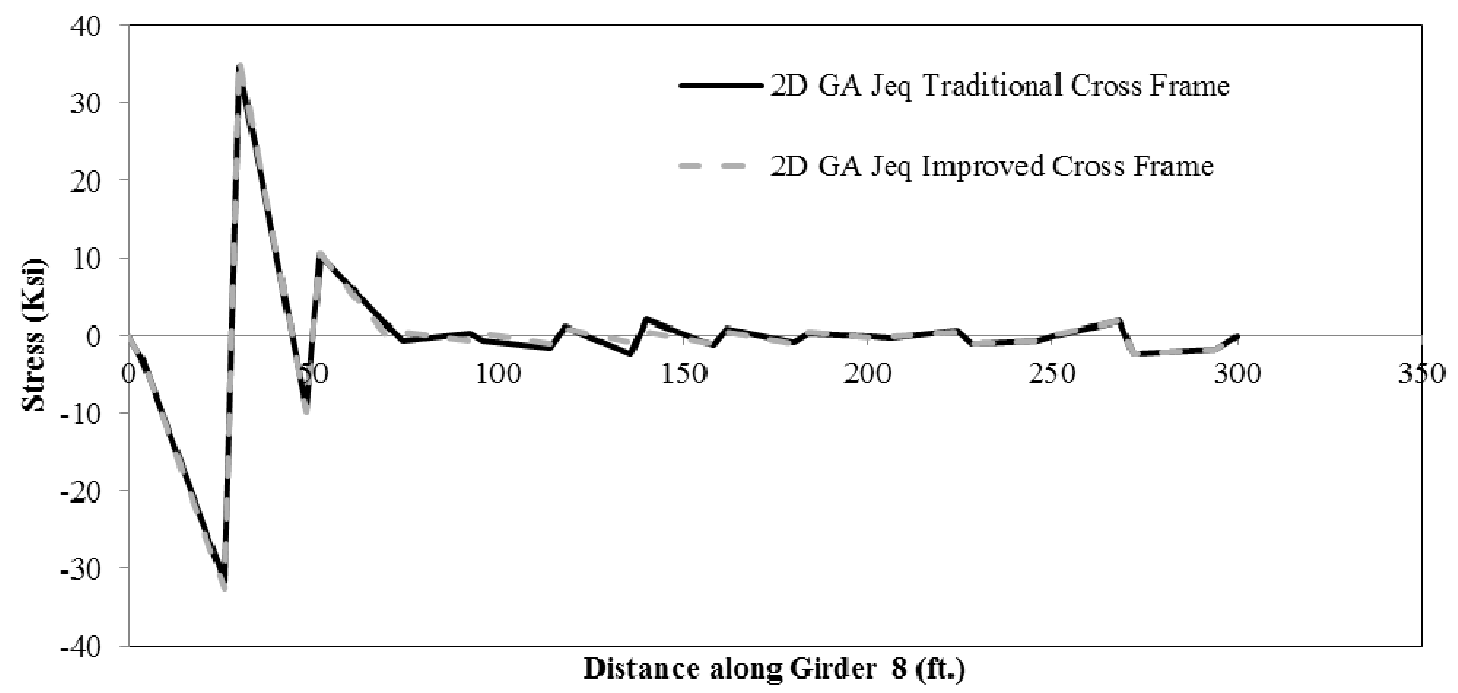

Flange lateral bending stress along length of girder 8 of Bridge A

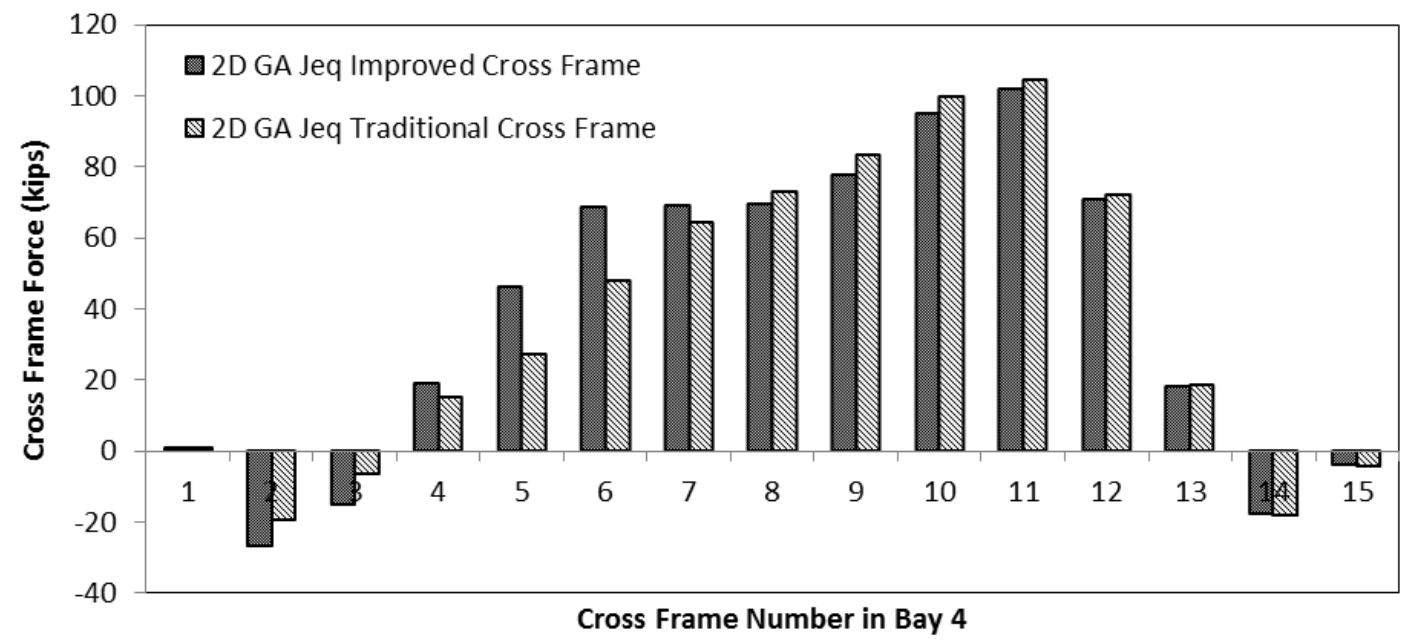

Cross frames forces in bottom chord of cross frames in bay 4 of Bridge A 


\section{C.4 Concrete Dead Load Deflections}

\section{C.4.1 Bridge A}
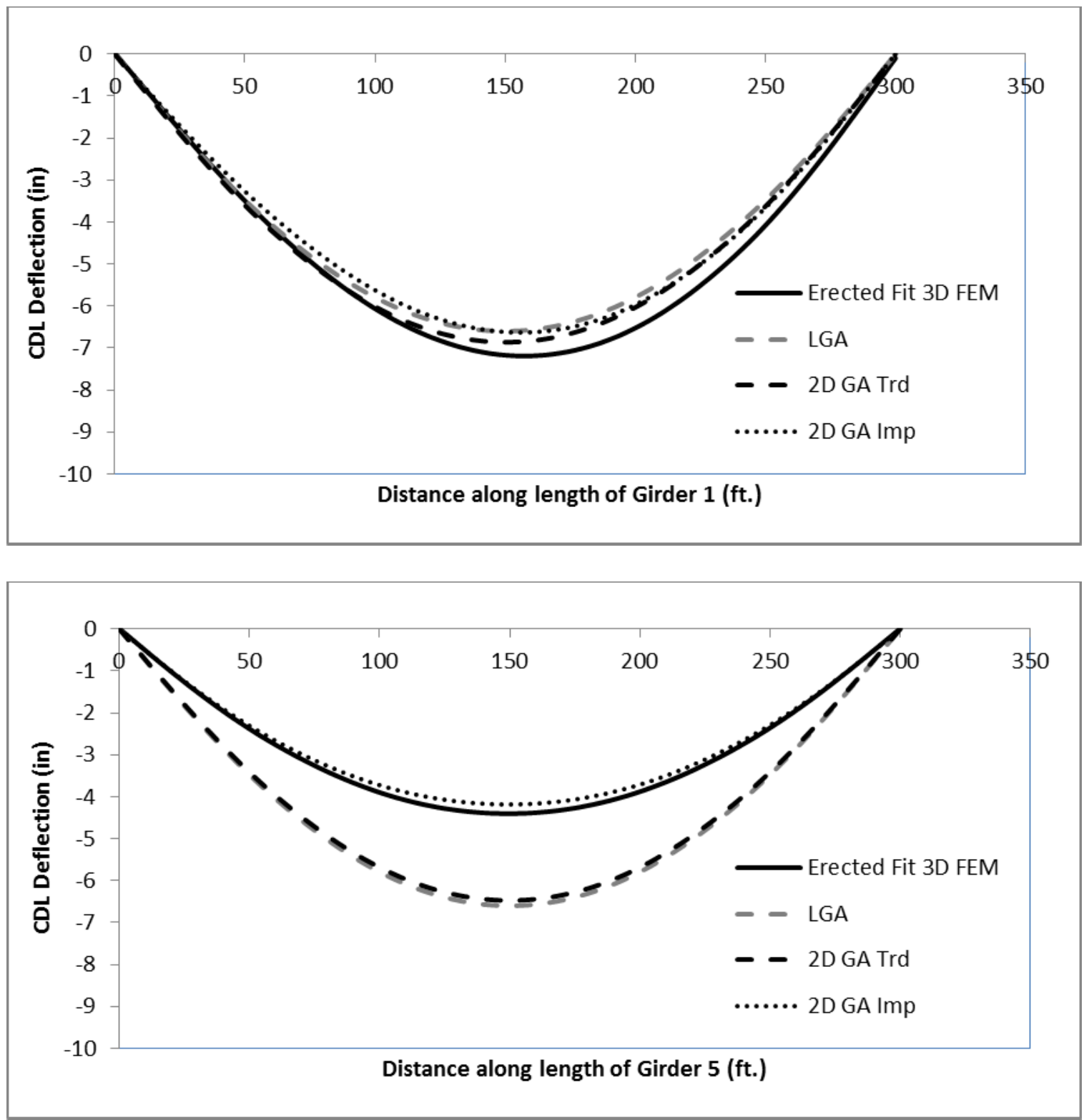


\section{C.4.2 Bridge B}

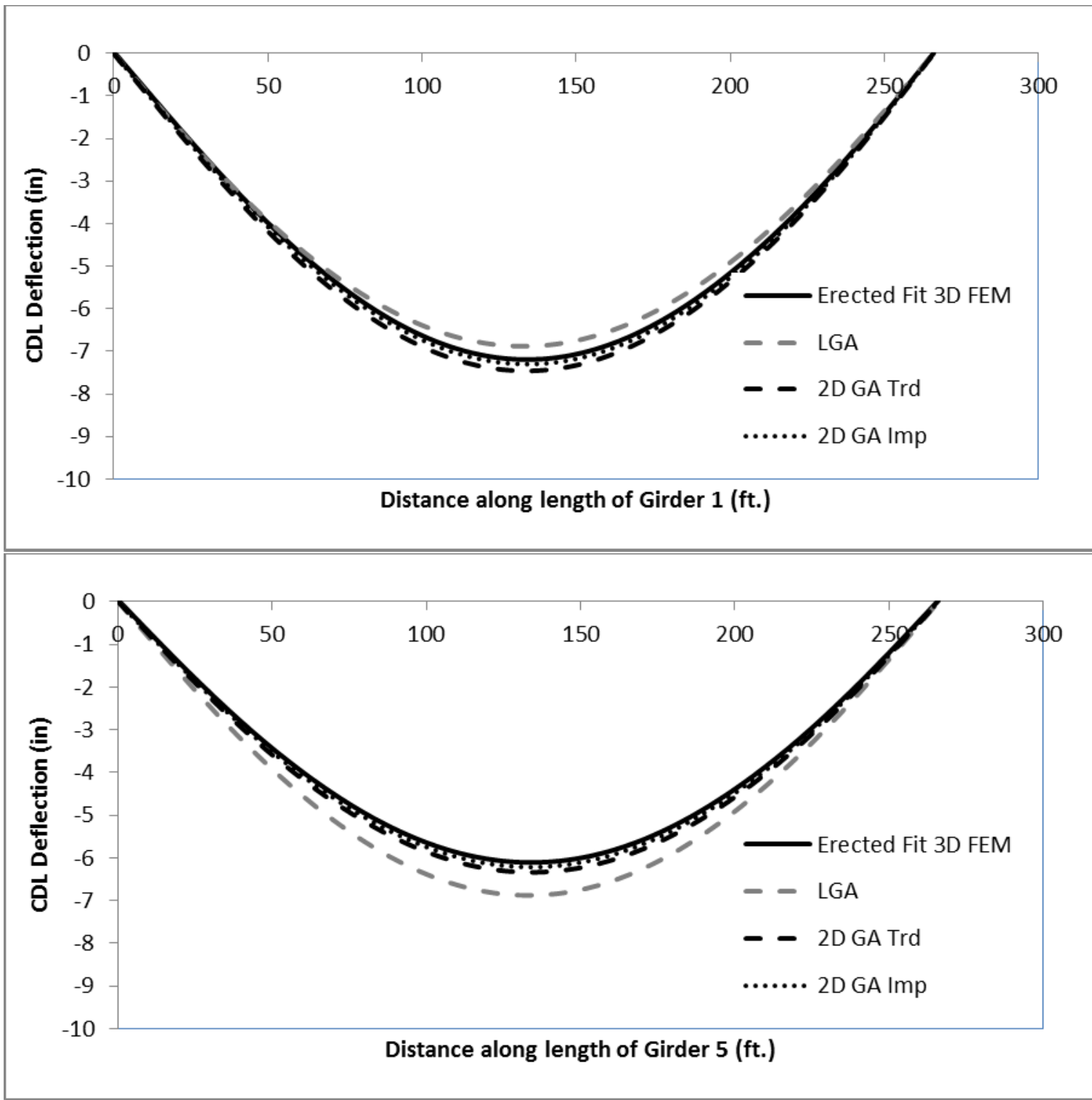




\section{C.4.3 Bridge C}
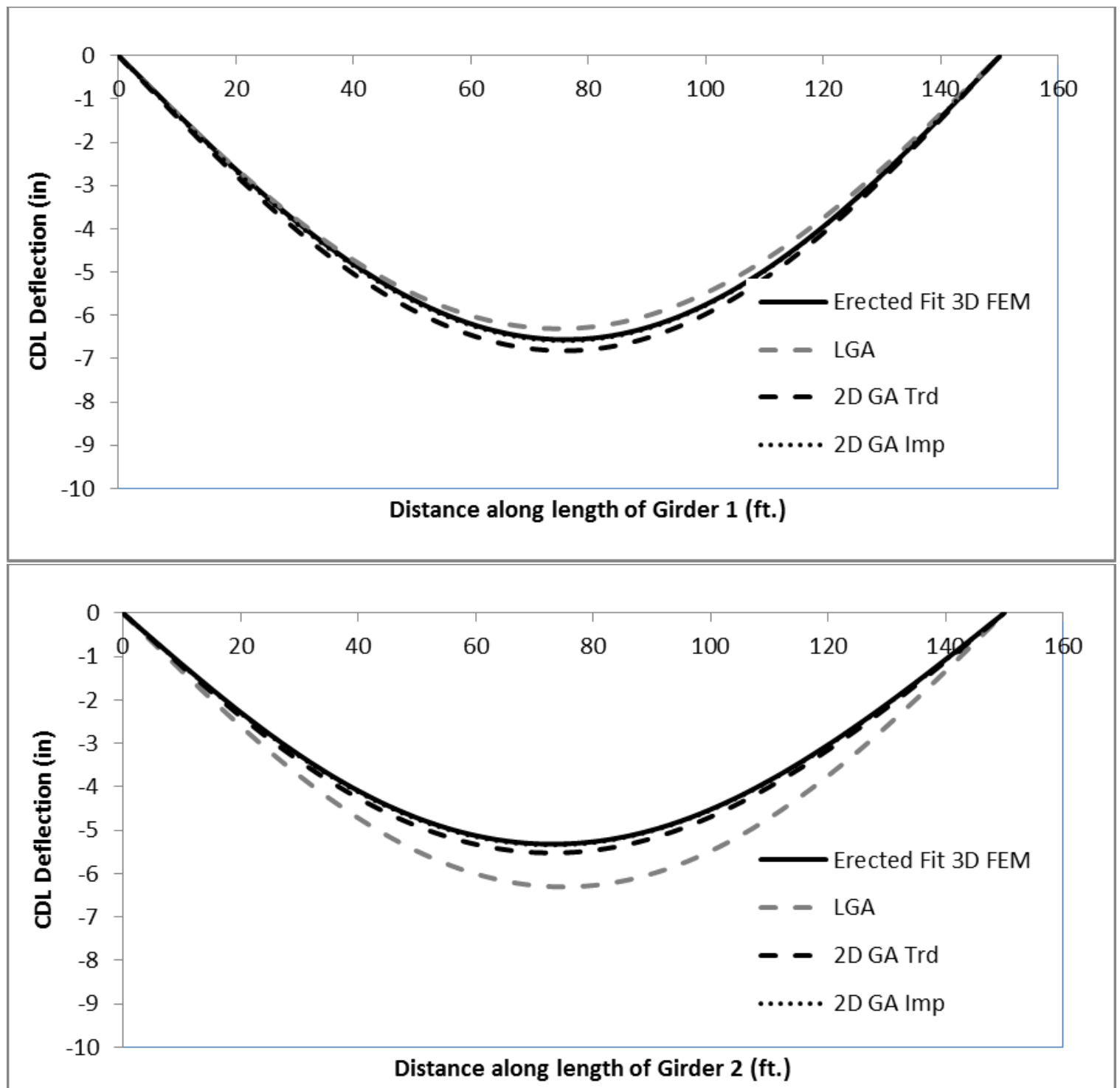


\section{C.5 Layovers}

\section{C.5.1 Bridge A}
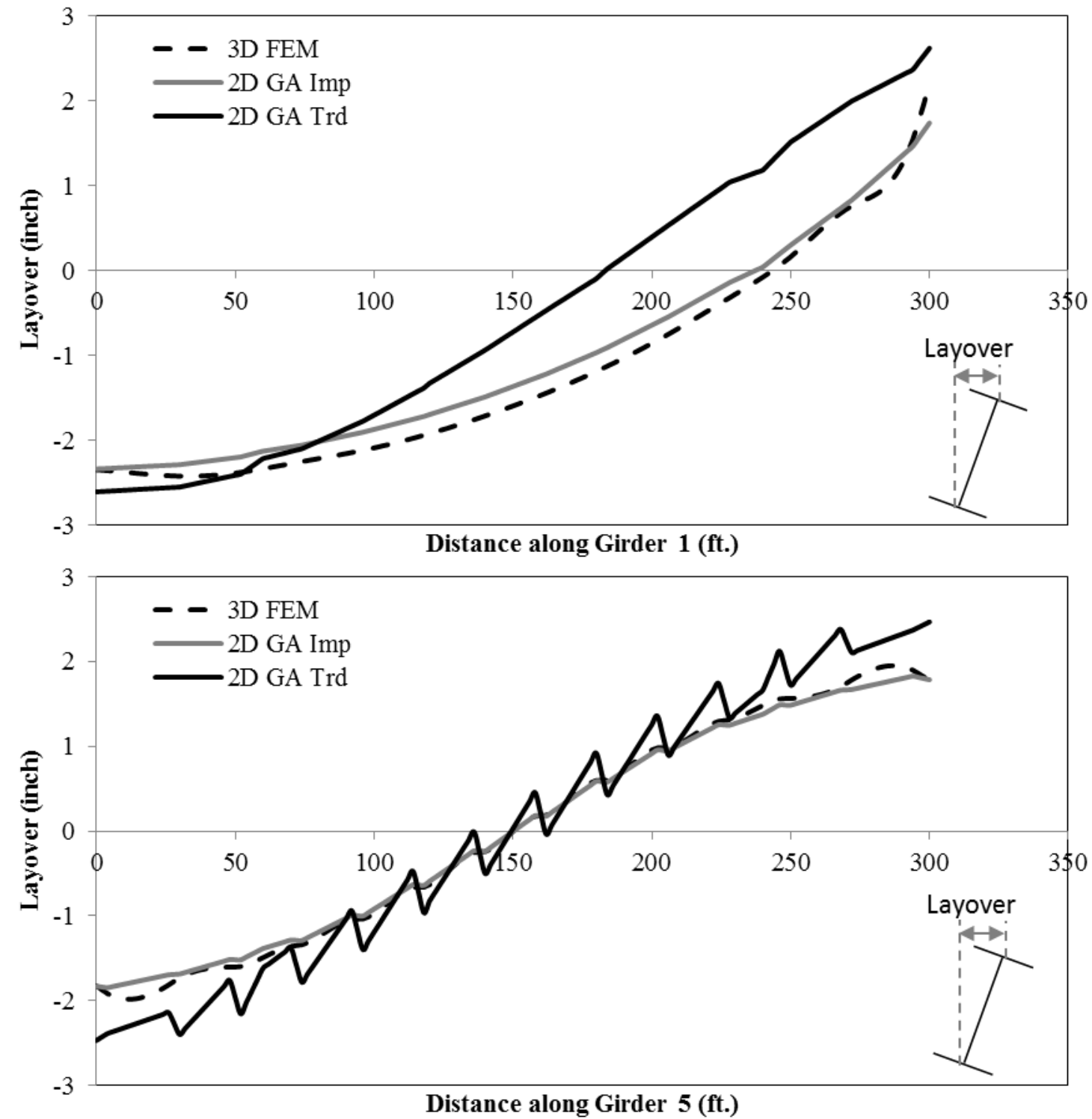


\section{C.5.2 Bridge B}
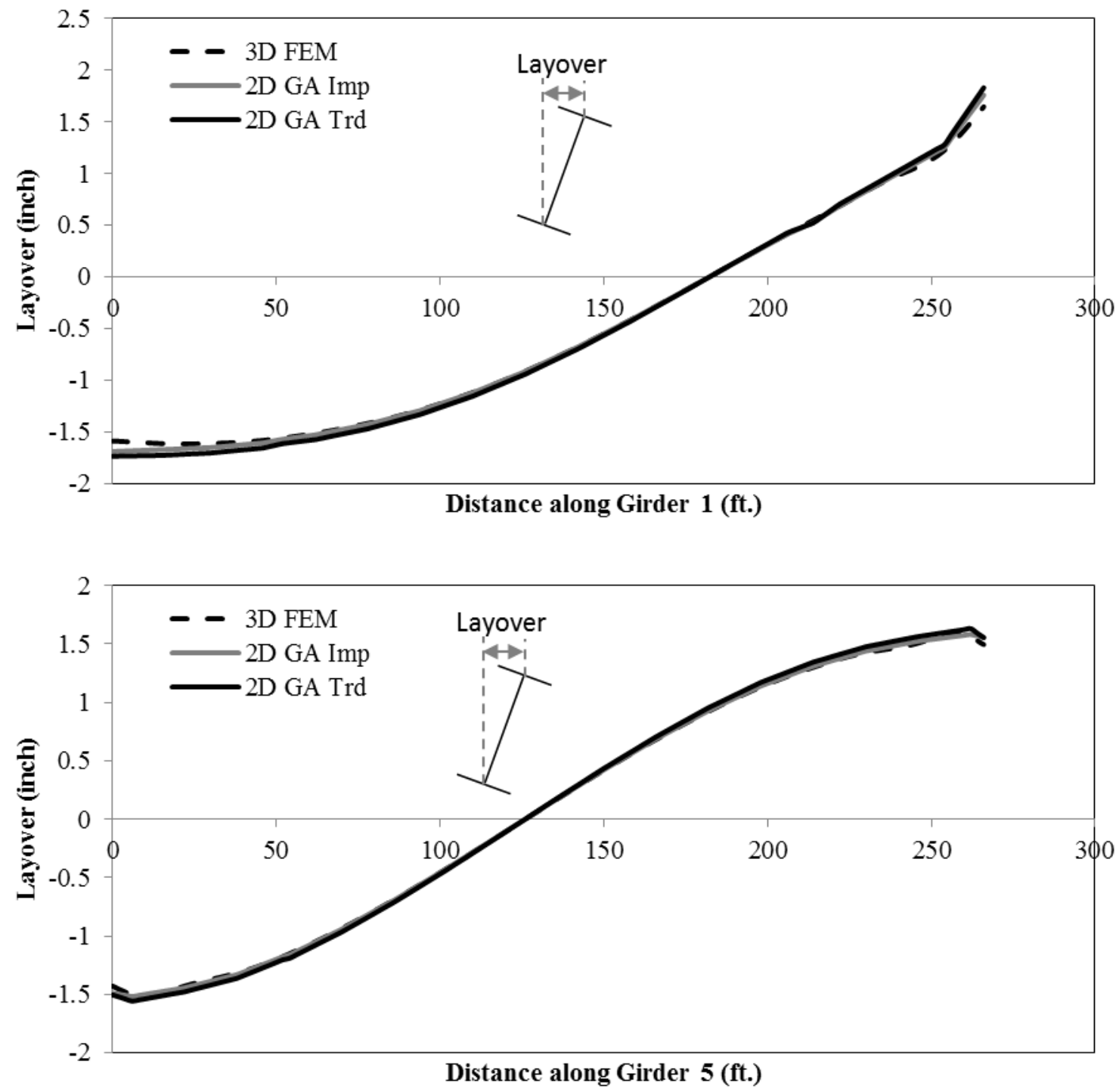


\section{C.5.3 Bridge C}
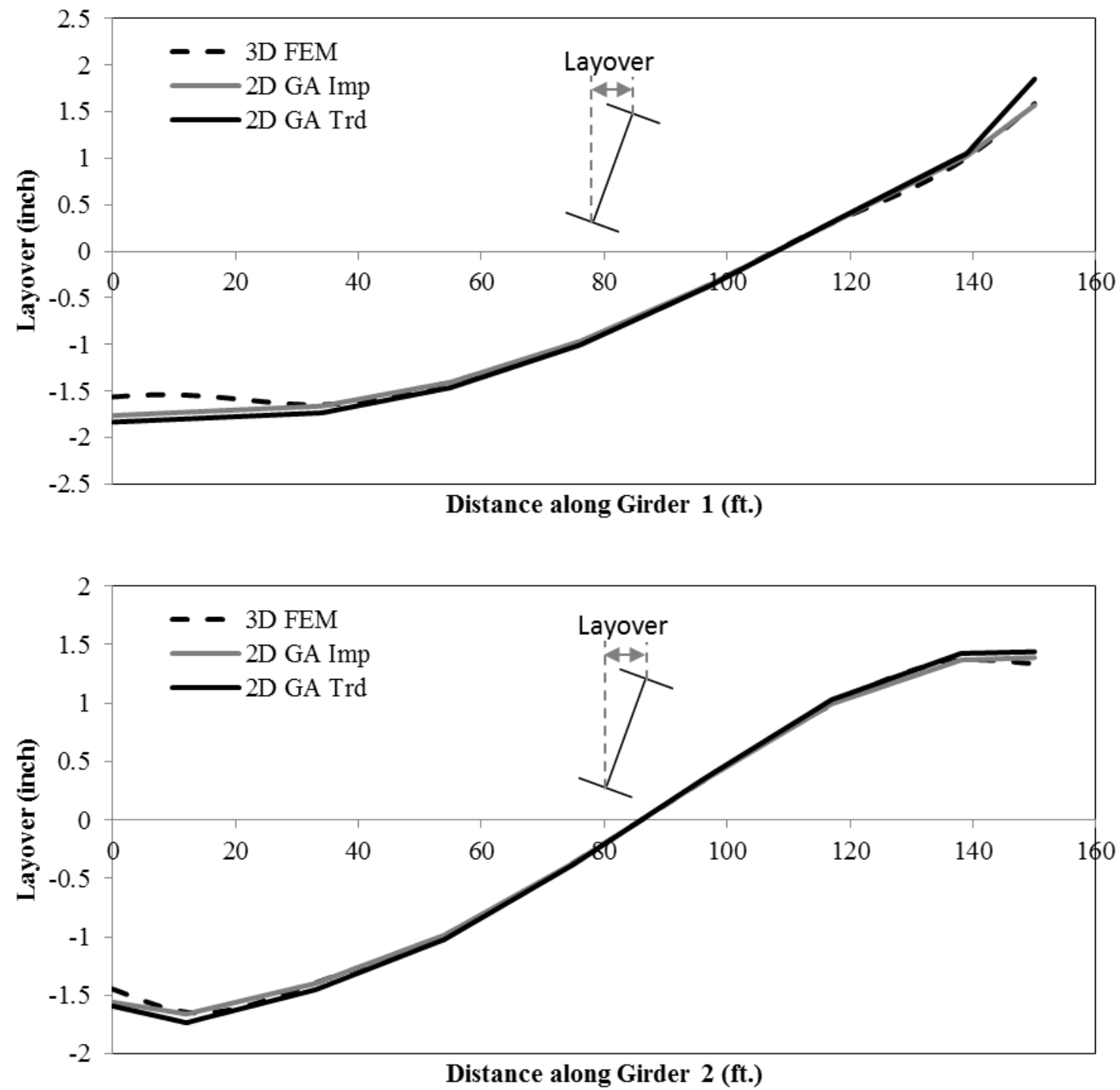


\section{C.6 Flange Lateral Bending Stress}

C.6.1 Bridge A
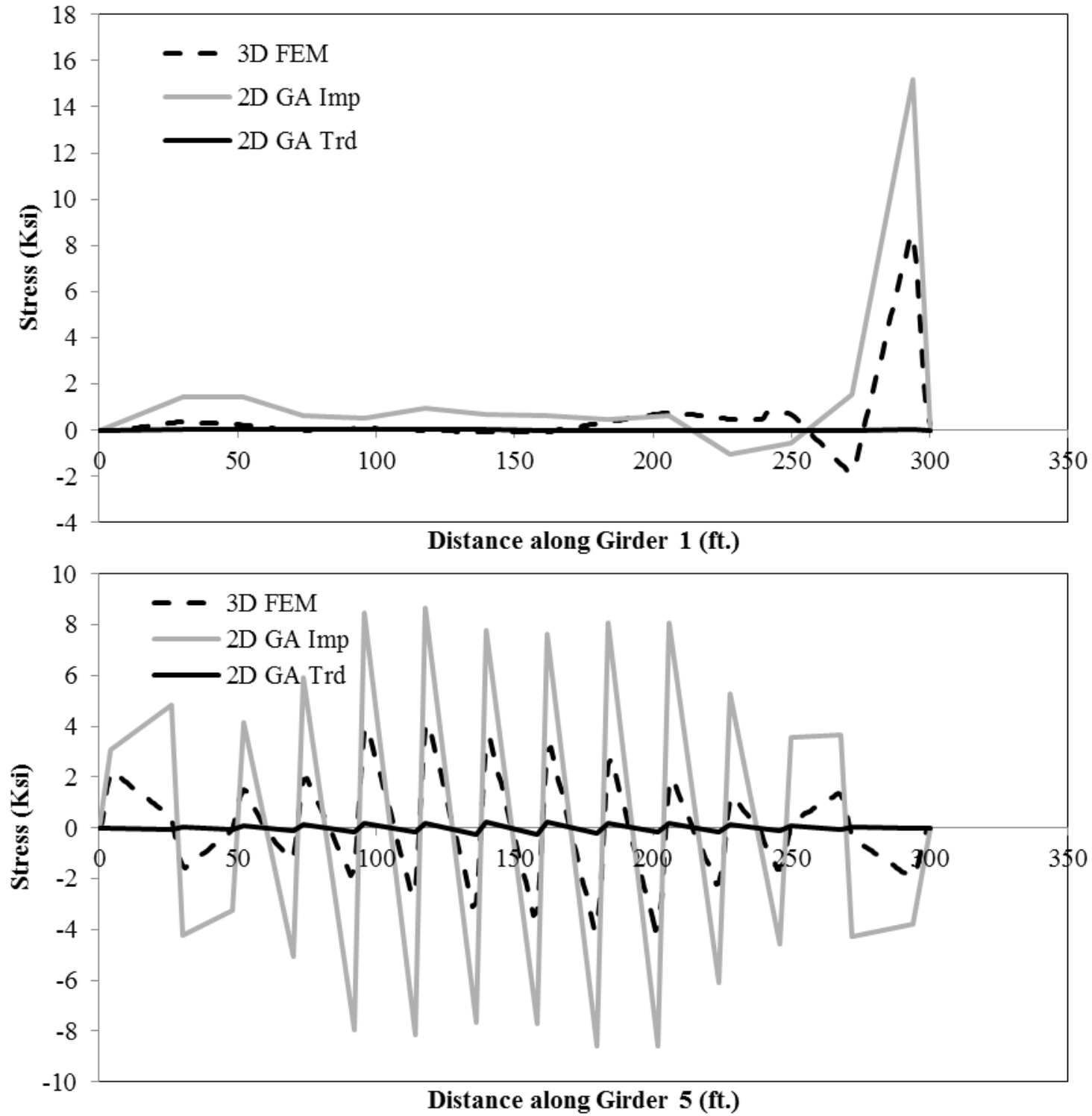
C.6.2 Bridge B
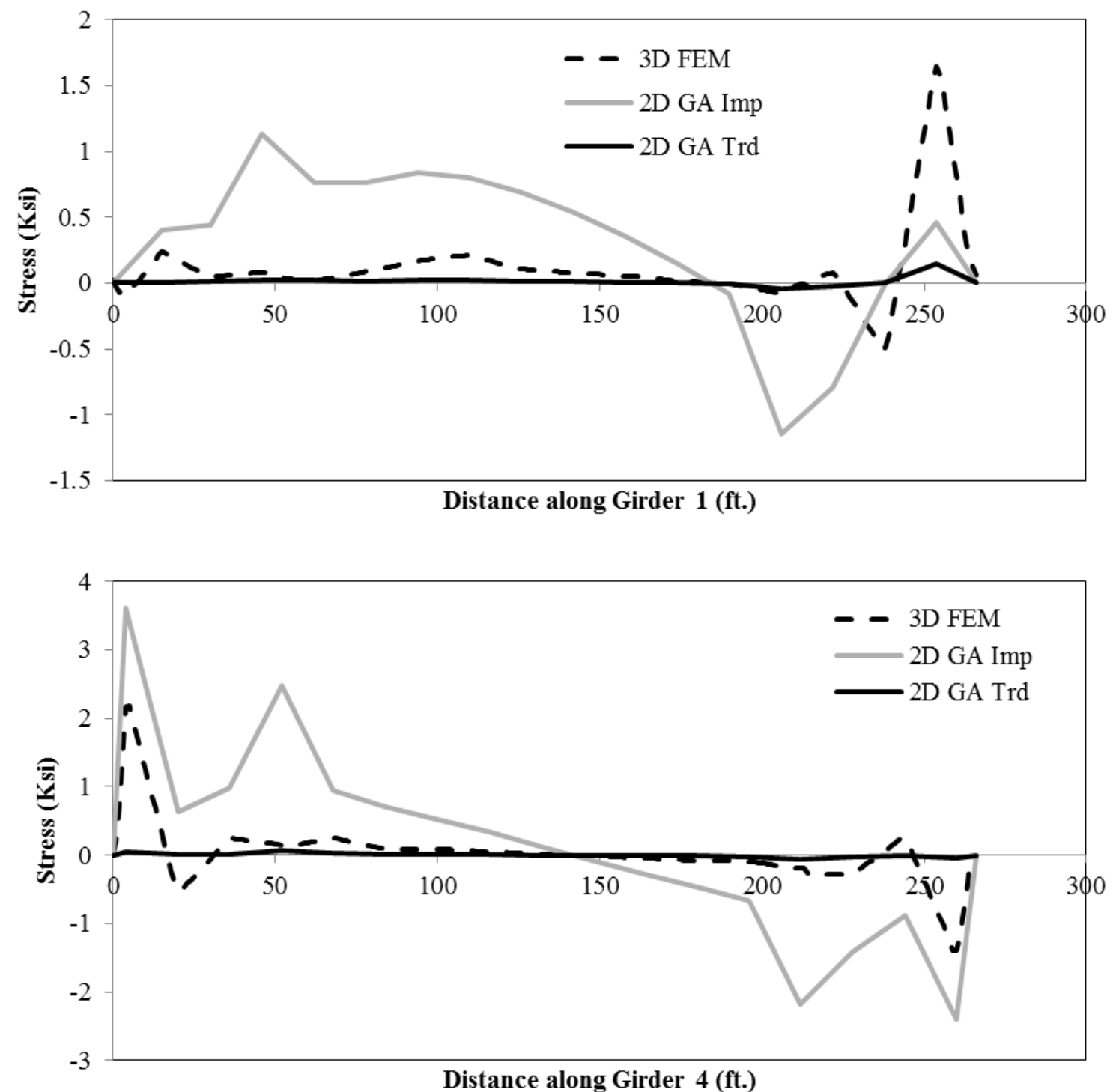


\section{C.6.3 Bridge C}
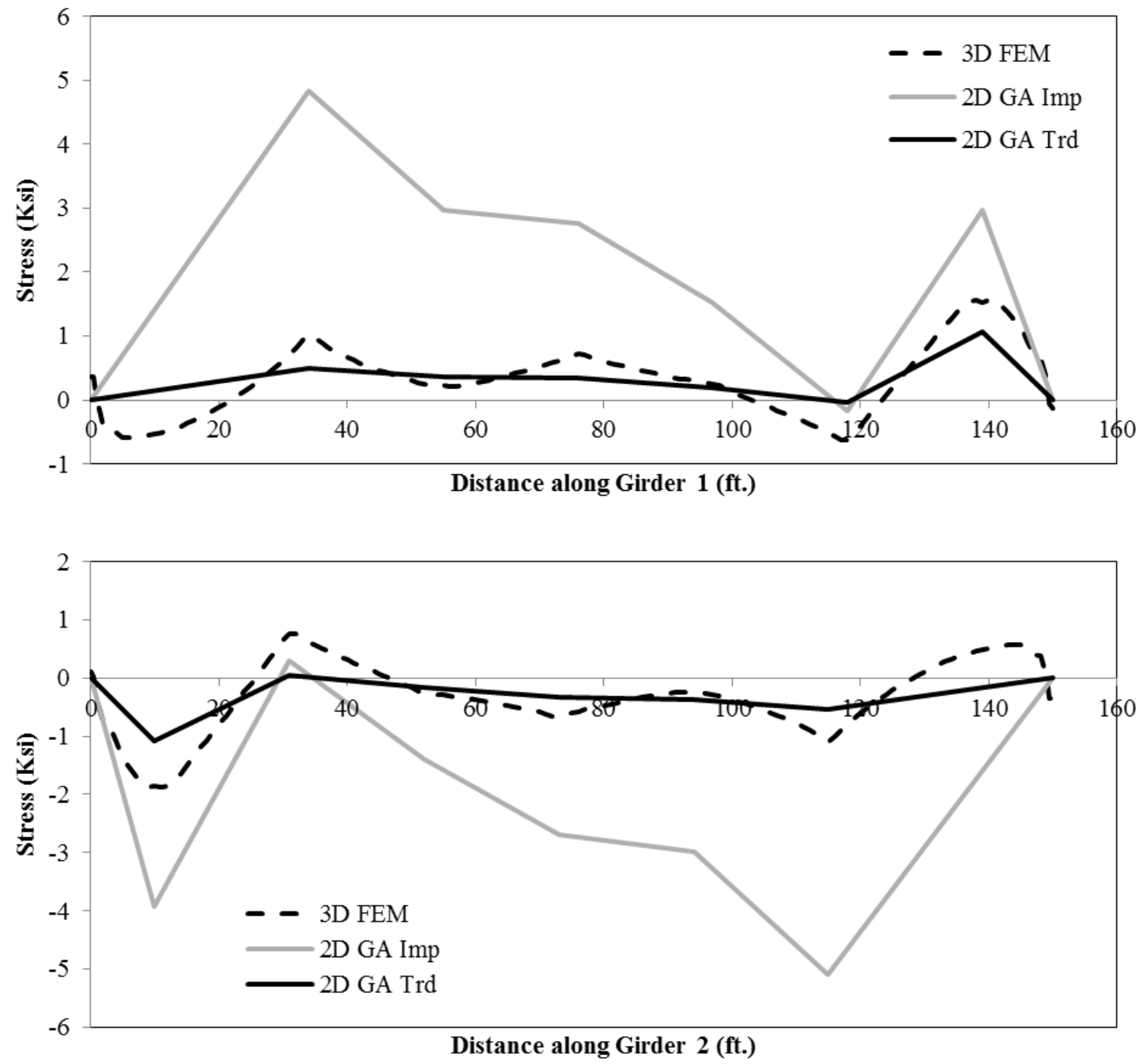


\section{C.7 Reactions}

\section{C.7.1 Bridge A}



\section{C.7.2 Bridge B}

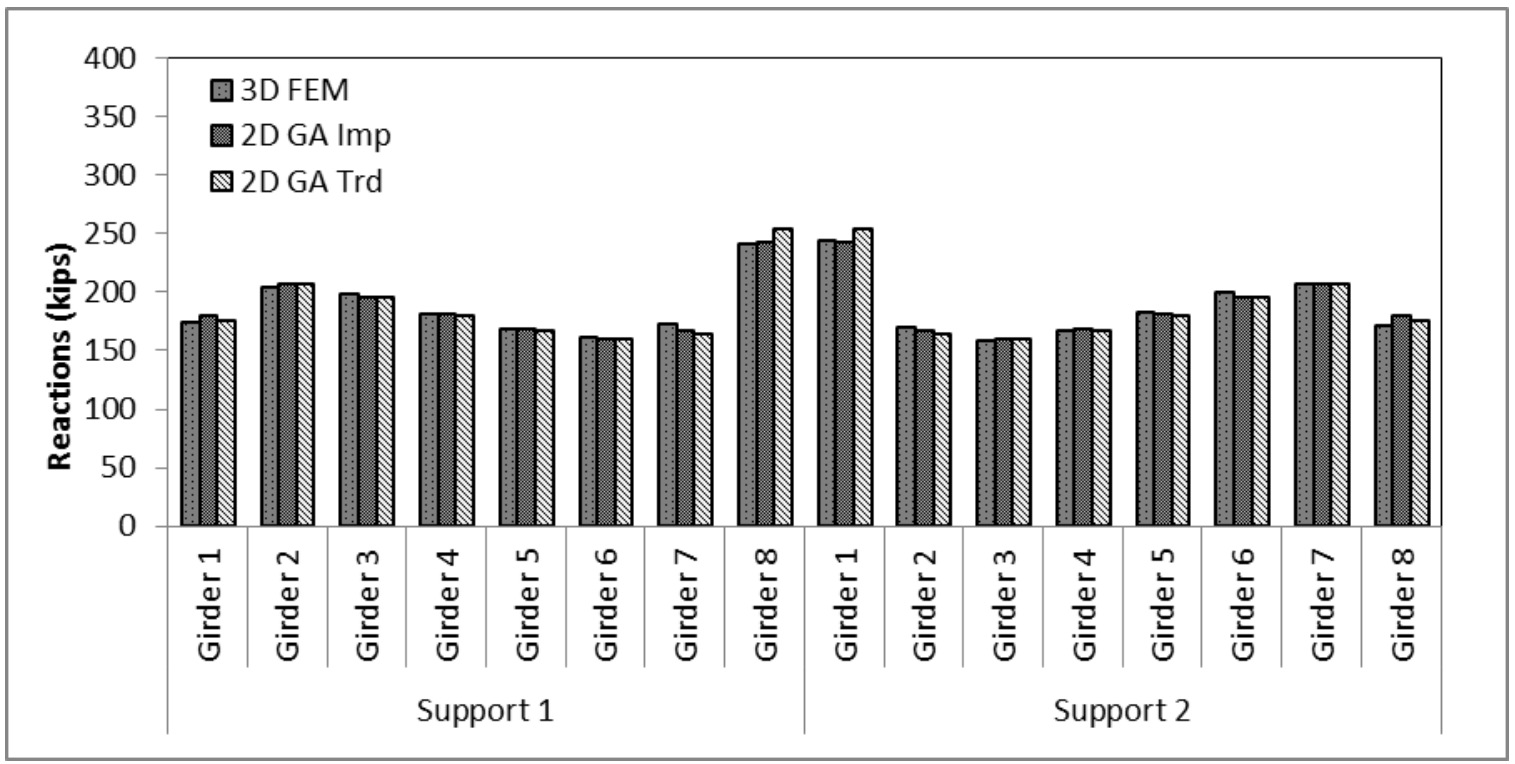




\section{C.7.3 Bridge $C$}

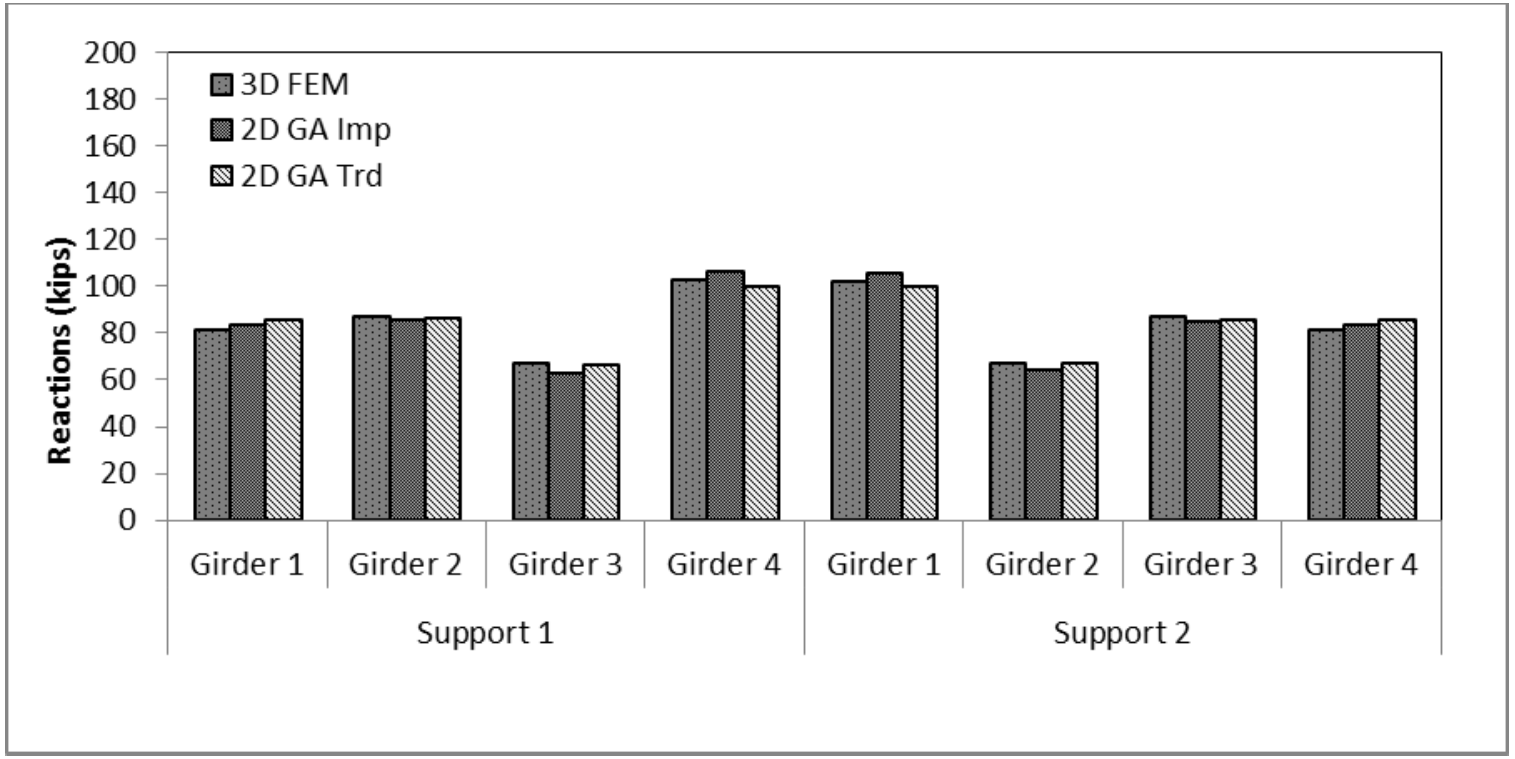




\section{C.8 Cross frame forces}

\section{C.8.1 Bridge A}
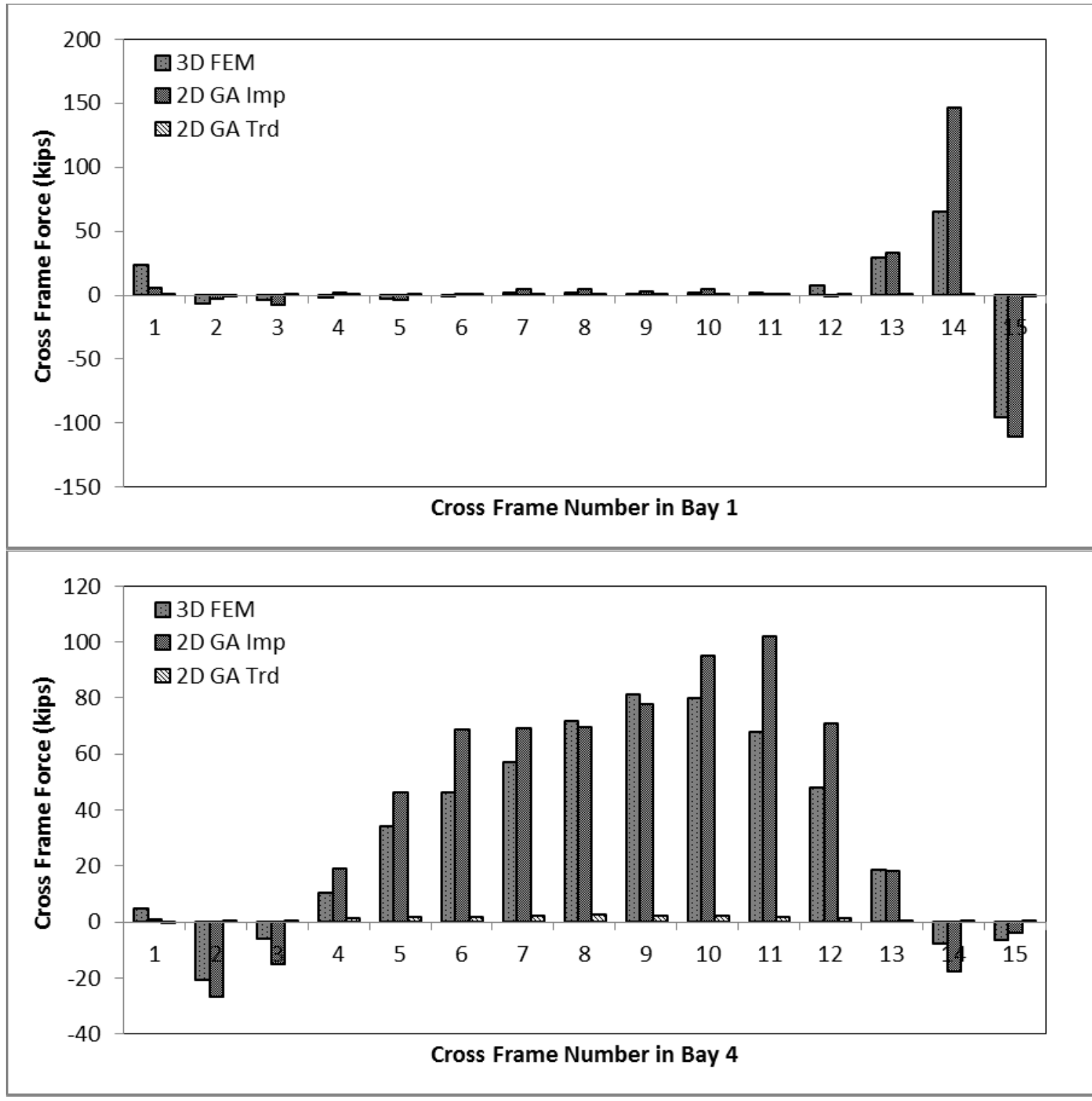
C.8.2 Bridge B

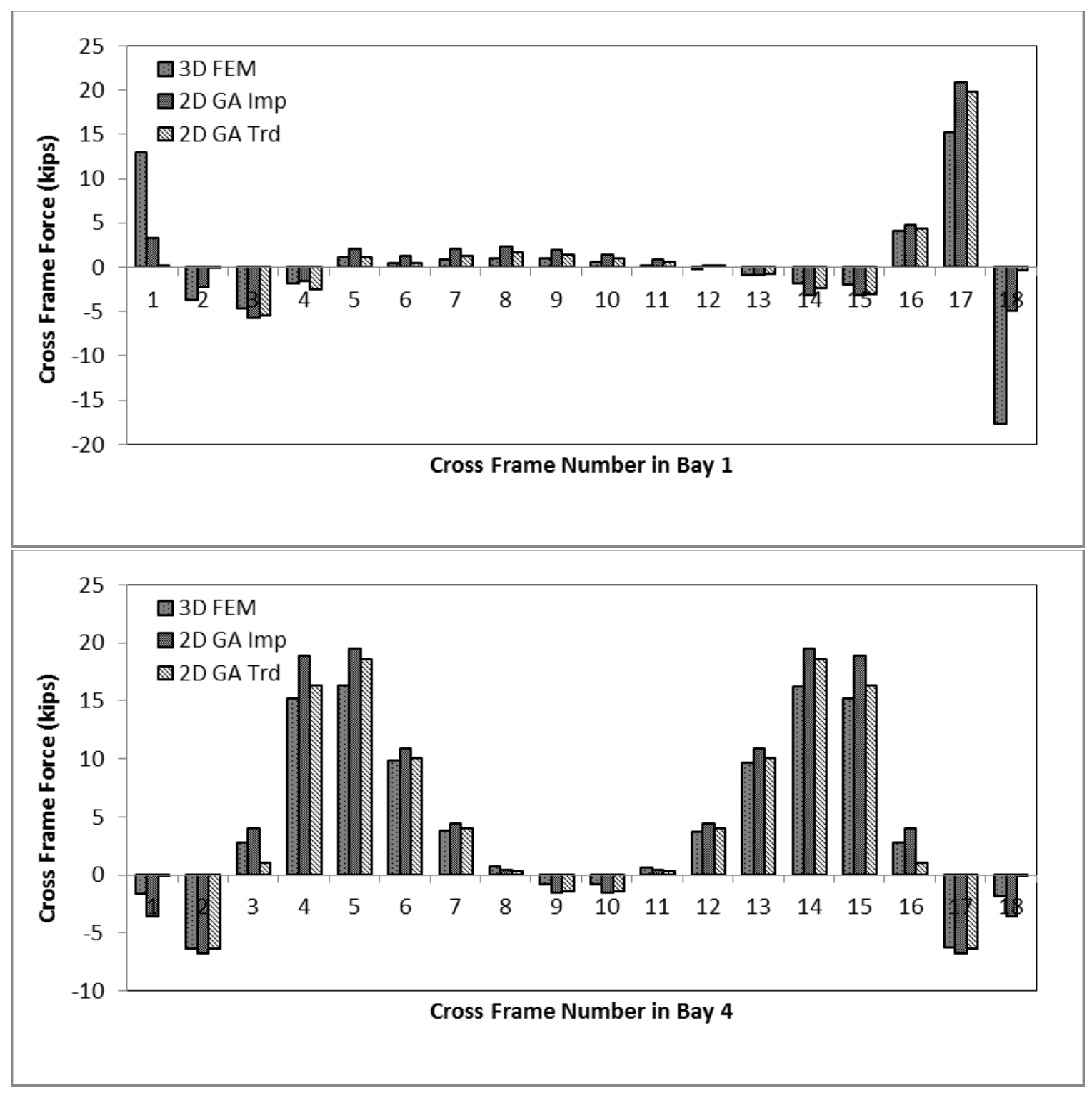




\section{C.8.3 Bridge C}

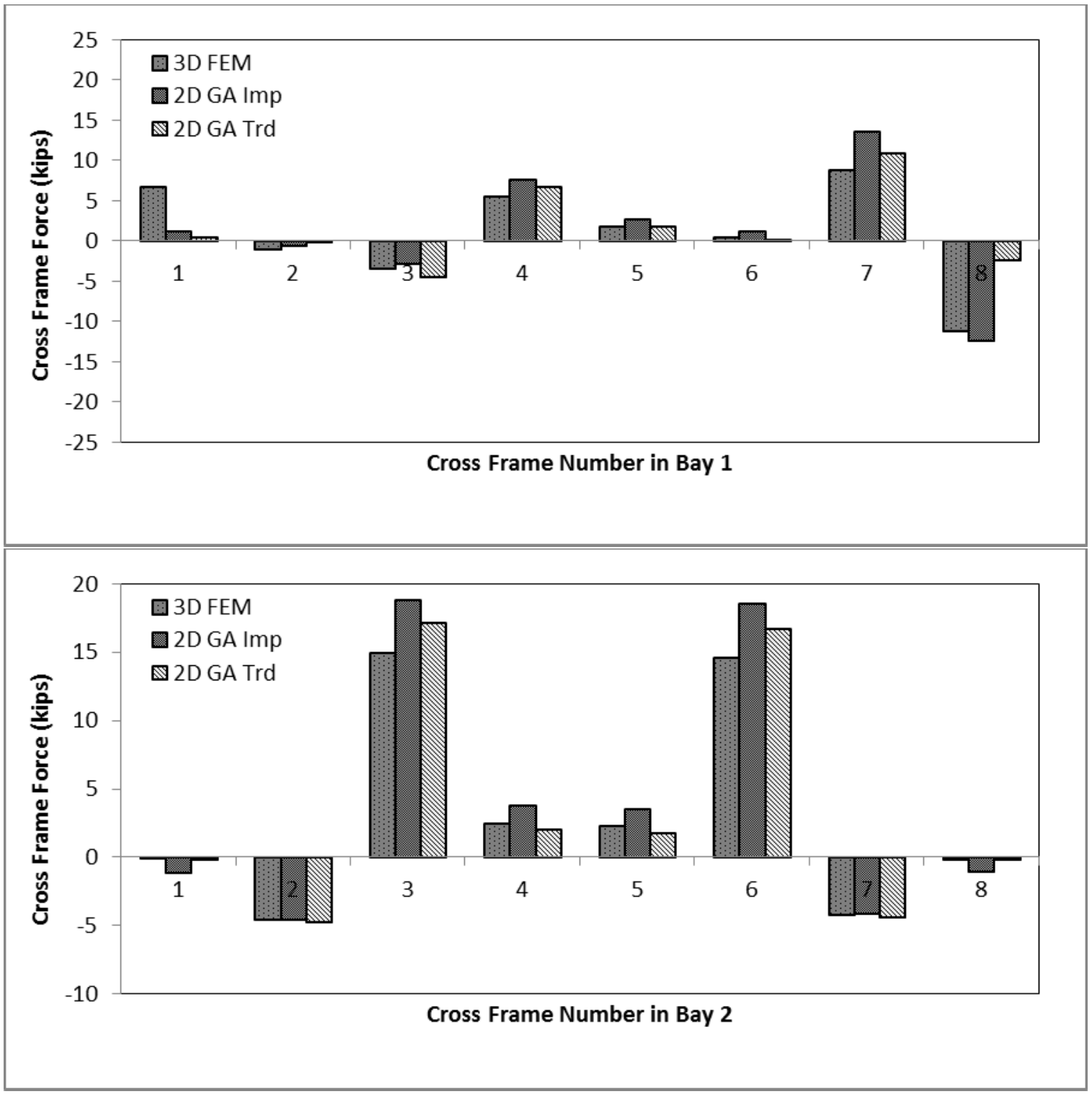




\section{APPENDIX D METHODS OF ANALYSIS- FINAL FIT}

\section{D.1 Change in Elevation Due to Lack of Fit}

D.1.1 Bridge C

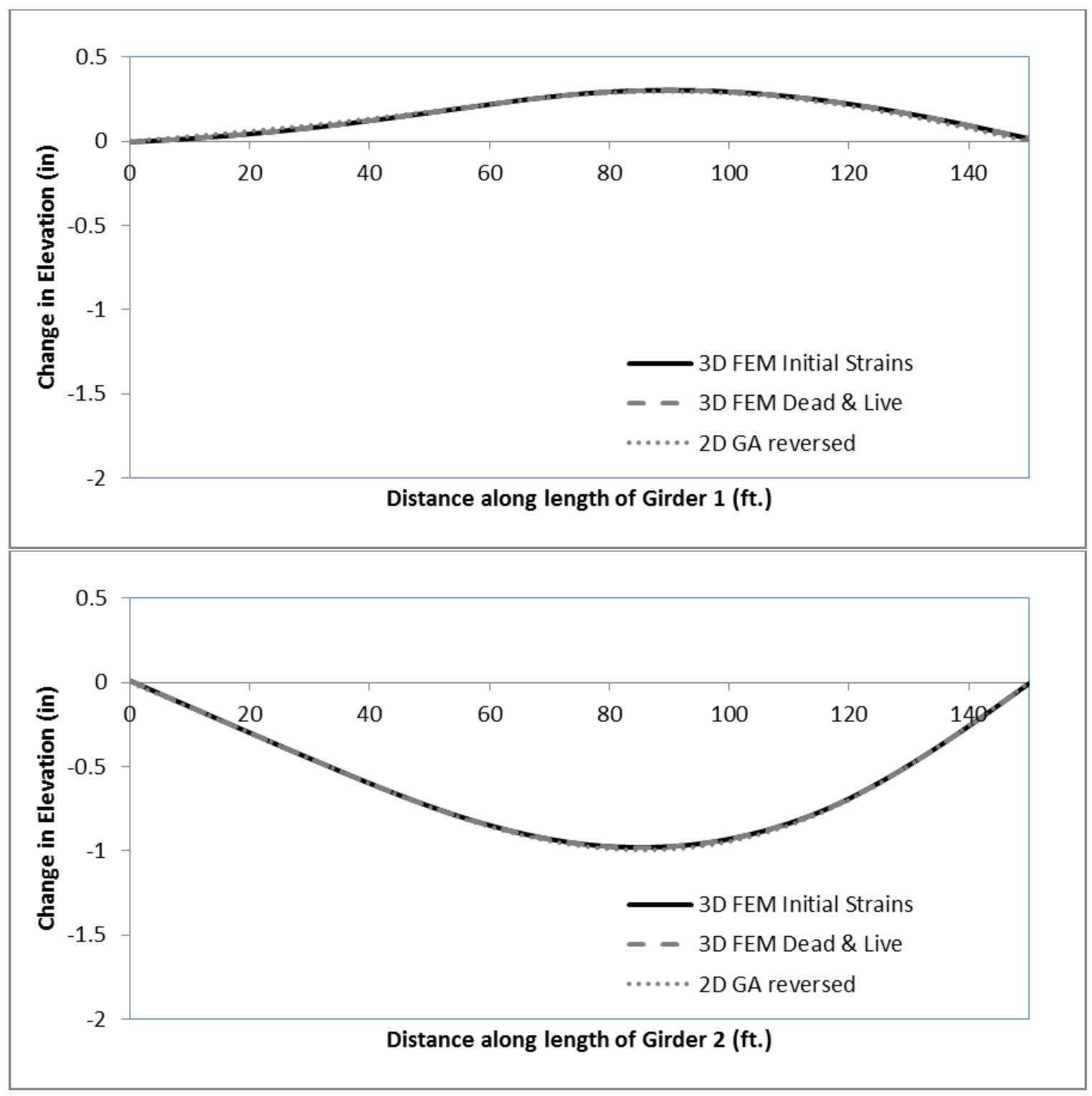




\section{D.2 Layovers}

D.2.1 Bridge C

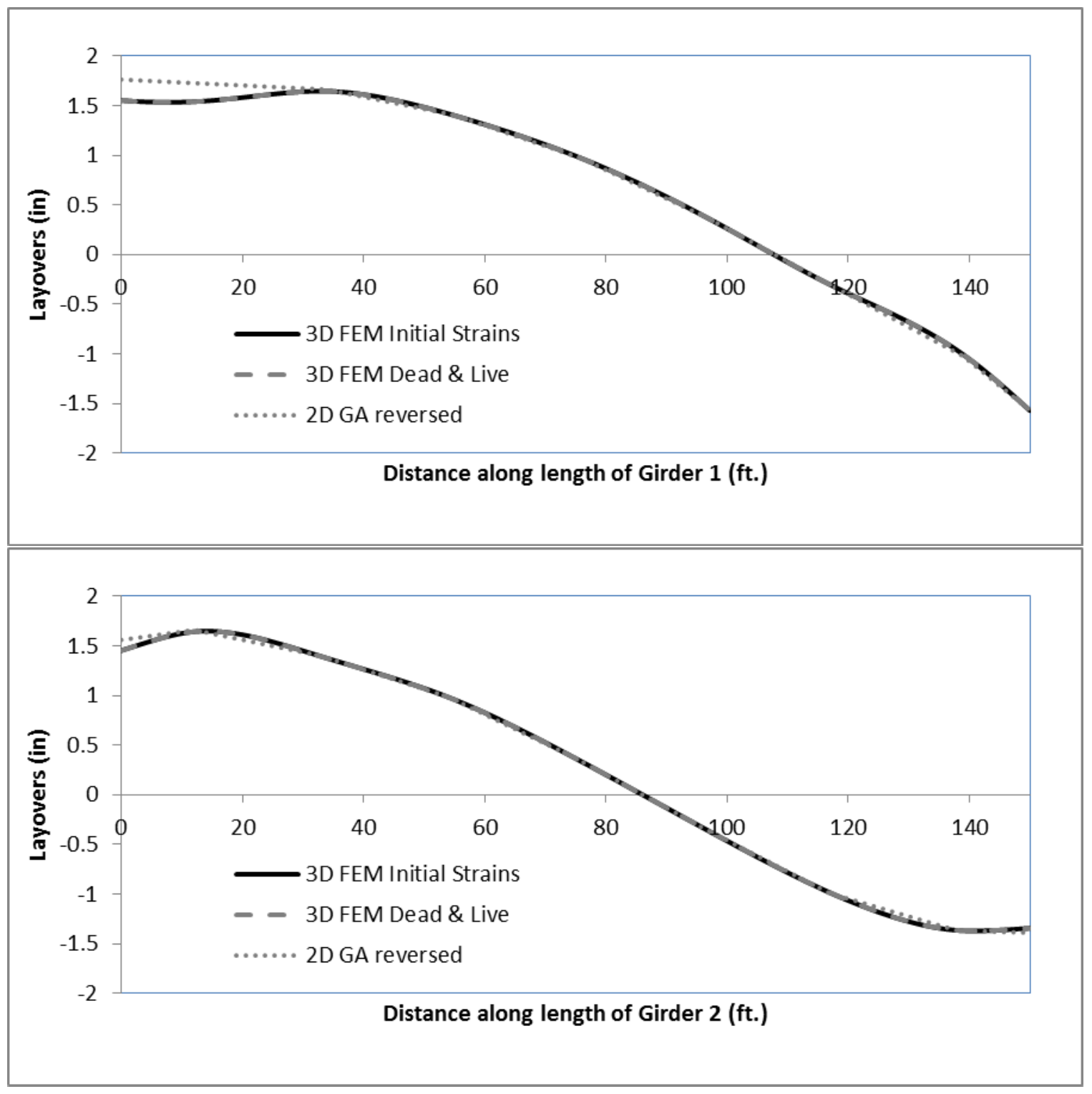




\section{D.3 Flange Lateral Bending Stress}

D.3.1 Bridge C

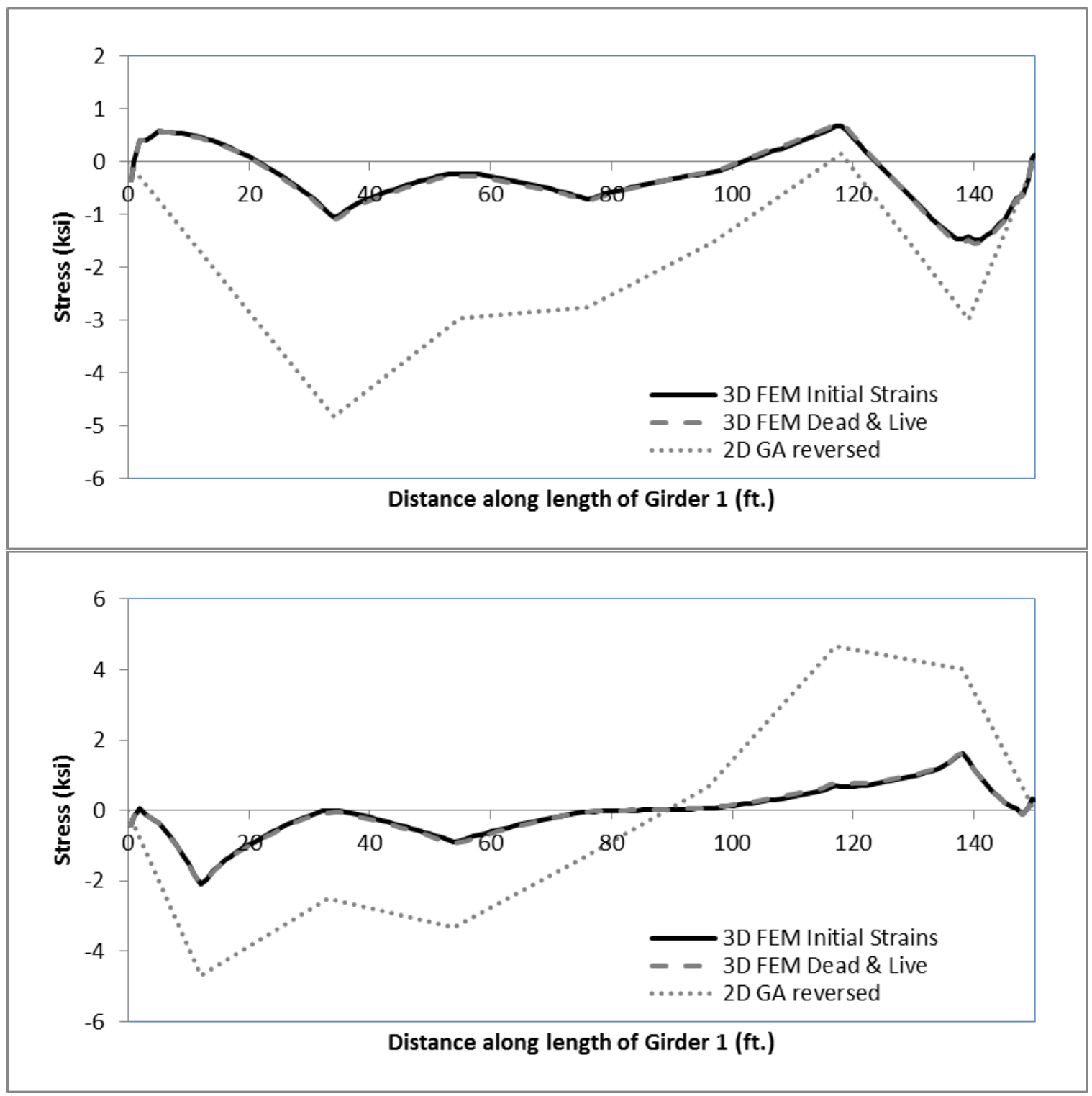




\section{D.4 Change in Reactions}

D.4.1 Bridge C

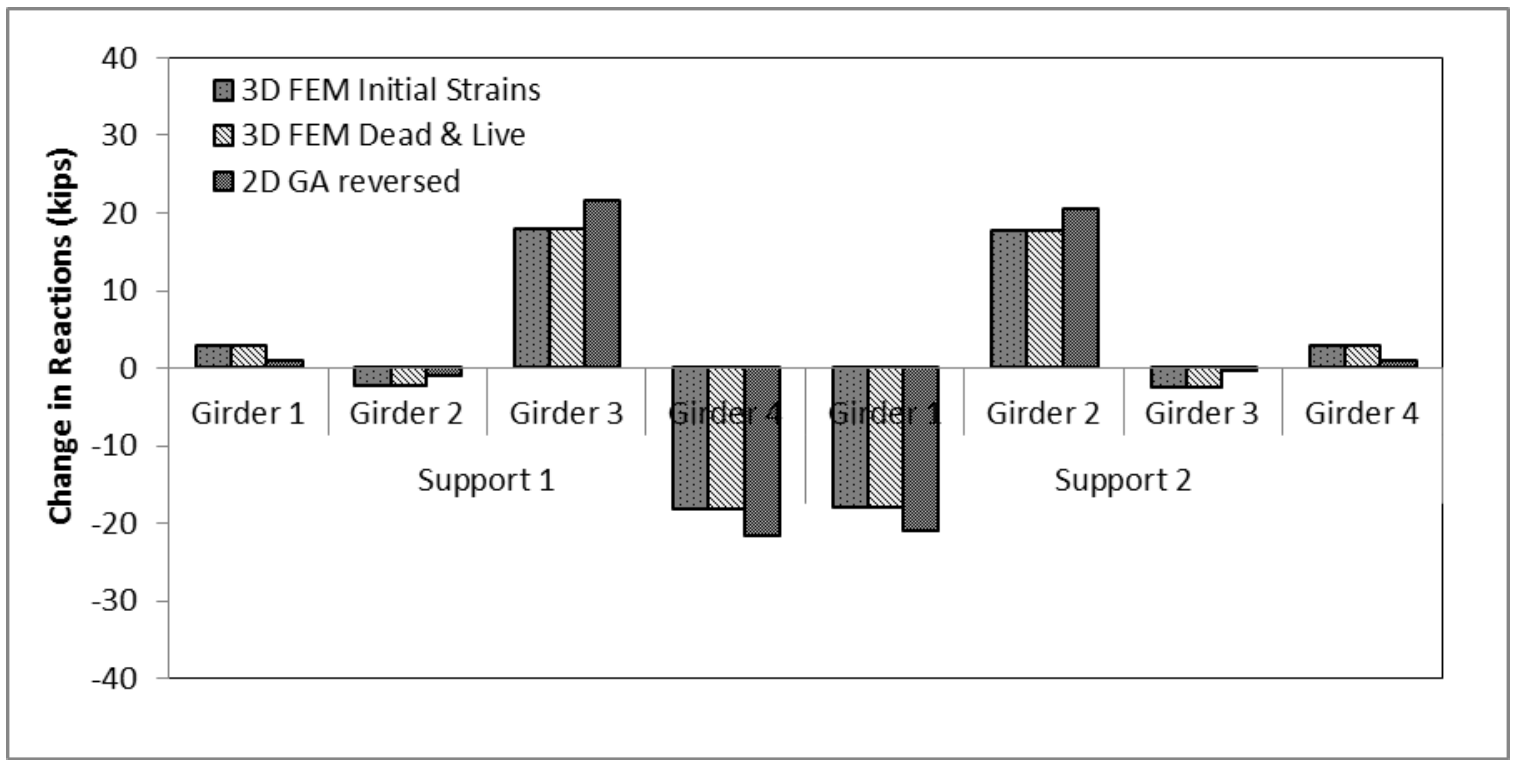




\section{D.5 Cross frame forces}

\section{D.5.1 Bridge C}
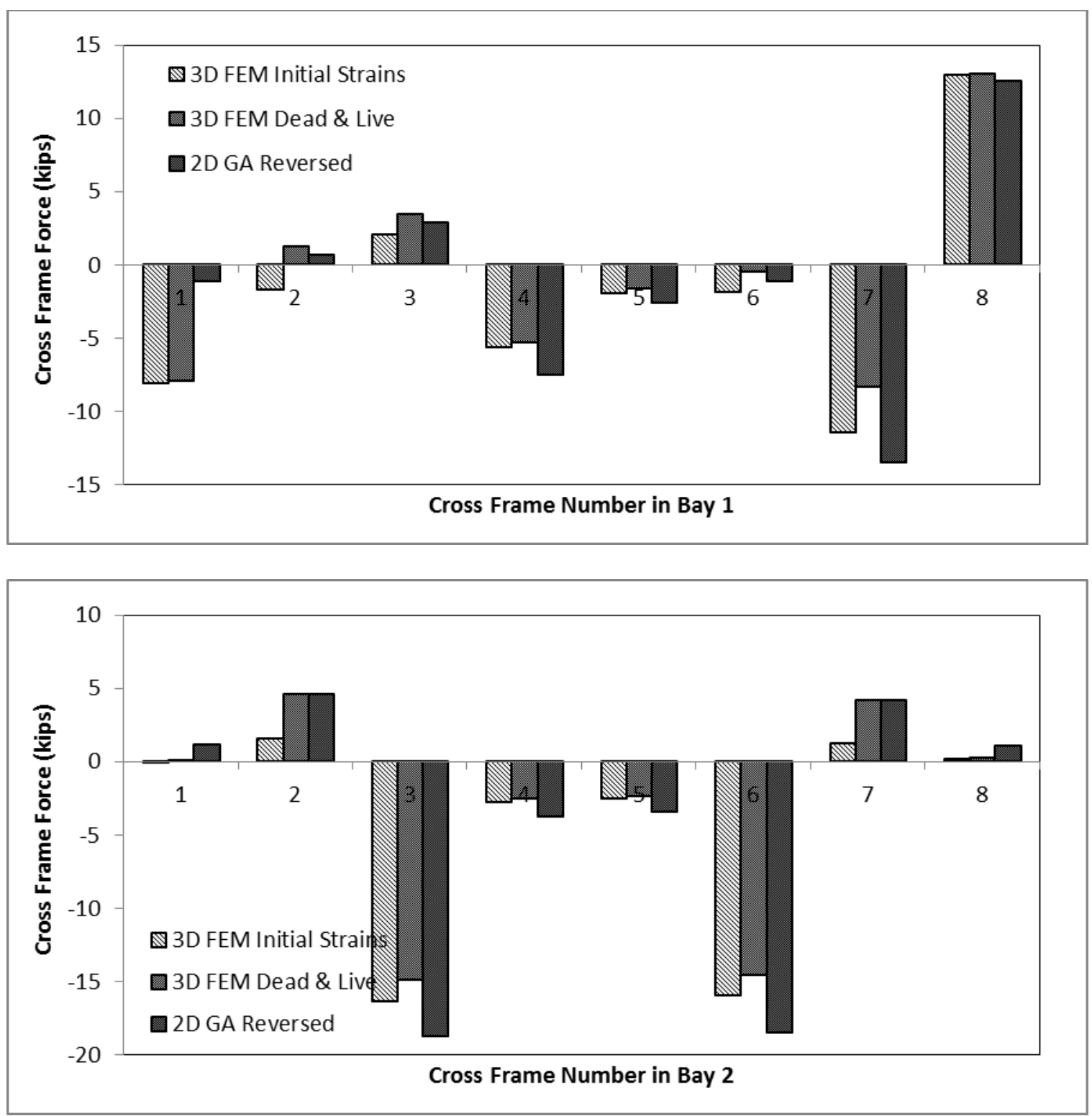


\section{APPENDIX E CONCEPTUAL EXAMPLE ON LACK-OF-FIT}

\section{E.1 Problem Description}

Two cantilever steel beams $10 \mathrm{ft}$. long are $10 \mathrm{ft}$. apart center to center from each. Free ends of these beams are required to be connected by $9.5 \mathrm{ft}$. long column that does not fit between its connections to free ends of the beams as shown in Figure E.1. Find out the force required to connect the column to beams. Find the stress in the column and beams after connection is made.

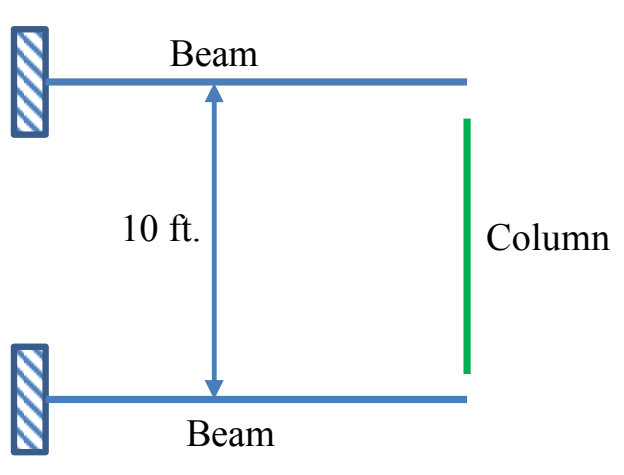

Length of both Beams $=10 \mathrm{ft}$.

Length of column=9.5 ft.

Modulus of Elasticity of Beams and Columns $=29,000 \mathrm{ksi}$

NOTE: Everything remains elastic at every stage

\section{Figure E.1: Conceptual problem describing lack-of-fit}

Simple square cross section is assumed for both beams and the column as shown

in Figure E.1. Both beams and the column are made of steel with modulus of elasticity of $29000 \mathrm{ksi}$. It is assumed that everything remains elastic, connections are pin and centerline of column is connected to centerline of beams. 


\section{E.2 Analytical Solution}

A two-step procedure is assumed to find out the force required to make the connection (Fit-Up force) and stress in the column and beams.

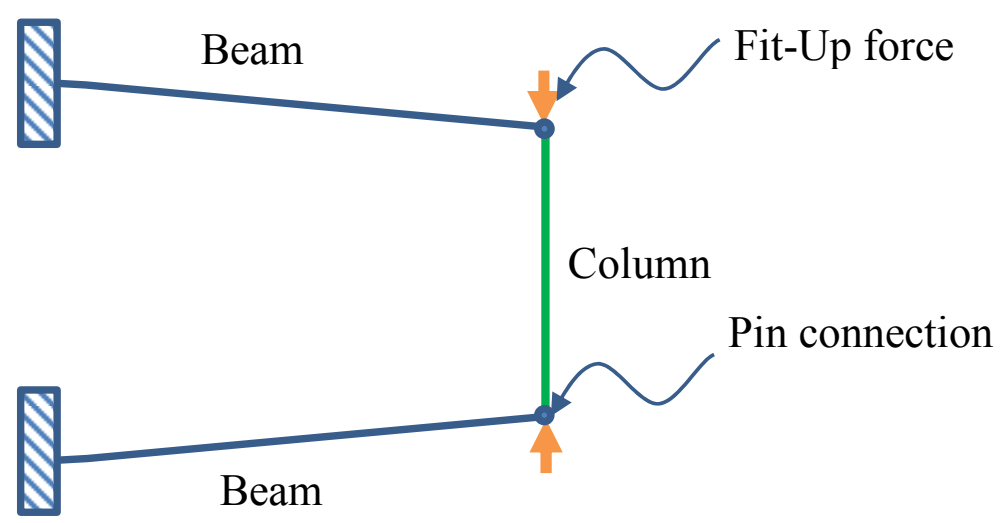

Figure E.2: Fit-Up force required to make the connections

Fit-Up force required to make the connection can be calculated by the beam deflection formula.

$$
P=\frac{3 E I \delta_{\text {beam }}}{L_{\text {beam }}{ }^{3}}=\frac{3 \times 29,000 \times 6.75 \times 3}{120^{3}}=1.02 \mathrm{kip}
$$




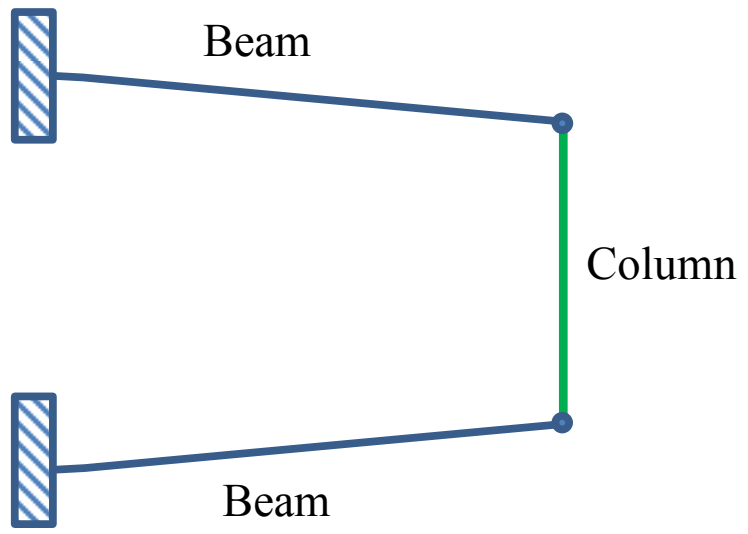

Figure E.3: Stress in beams and column once connections are made and Fit-Up force is removed

Once the connection is established the come along force is removed and the beams try to go back to their original position. Due to this fact the beams apply a force to the column that is equal and opposite to the come force. This force deform the column axially and decrease the $\delta_{\text {beam }}$.

$$
\delta_{\text {column }}=\frac{P L_{\text {column }}}{A E}=\frac{1.02 \times 114}{1 \times 29,00}=0.004 \mathrm{in} .
$$

Axial deformation of column is very small so it shall not change the deflection of beam ( 3 in.) by large amount and so forth the force. Therefore, the final stresses can be calculated as follows.

$$
\begin{gathered}
\sigma_{\text {beam }}=\frac{M C}{I}=\frac{1.02 \times 120 \times 1.5}{6.75}=27.2 \mathrm{ksi} \\
\sigma_{\text {col }}=\frac{P}{A}=\frac{1.02}{1}=1.02 \mathrm{ksi}
\end{gathered}
$$




\section{E.3 FEM Solutions}

\section{E.3.1 Using Initial Strains (ANSYS)}

In the FEM world column can be stretched to make the connection. The initial strain is put into the column equal to the amount of stretch to make the connections. This stretched state of the column is an imaginary high-energy state and the system is not in equilibrium. Once static analysis is run, column shrink back to its original length and equilibrium is established.



\section{Figure E.4: Initial strain in column to model lack-of-fit}

Notice that Final stretched length of the column is used to calculate the initial strain. This because the software multiply the initial strain with the modeled length of the column $(120 \mathrm{inch})$ to find out the stretch $(6 \mathrm{inch})$ in the column $(0.05 \times 120 \mathrm{inch}=6 \mathrm{inch})$.

Once the model is complete with appropriate initial strain in the column, the static analysis is run without any load applied to the system. Once static analysis is complete, the column shrink back to its original length and system establish the equilibrium attaining lowest energy state. 


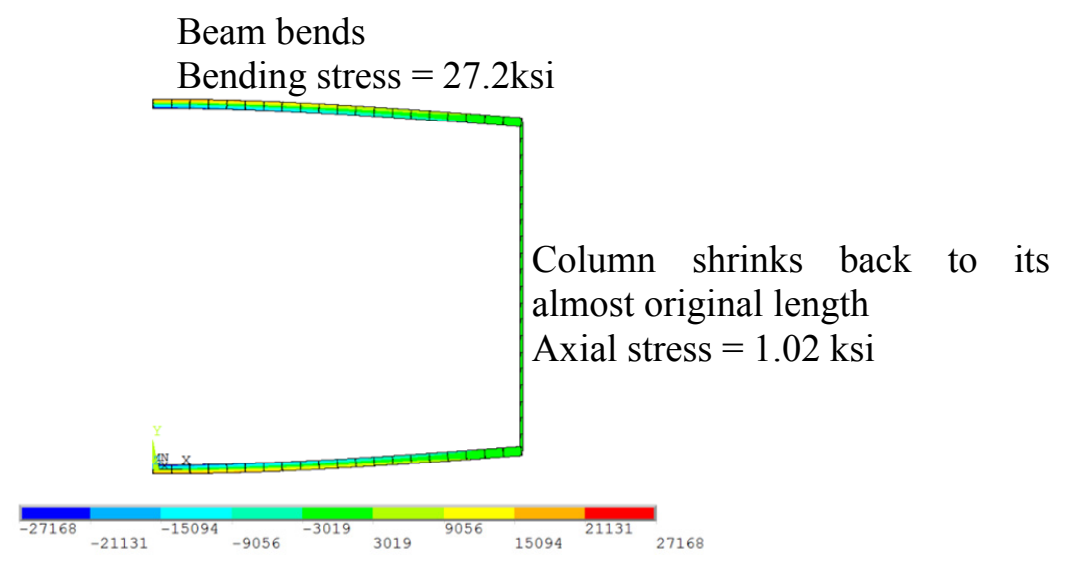

\section{Figure E.5: Equilibrated system after static analysis}

The stresses in columns and beams can be directly obtained by viewing the postprocessors. The fit-up force can be assumed as the force in column for this case. So the Fit-up force is $1.02 \mathrm{ksi} \times 1 \mathrm{in}^{2}=1.02$ kips.

\section{E.3.2 ANSYS code}




REAL,1

MAT,1

$\mathrm{K}, 1,0,0,0$

$\mathrm{K}, 2,120,0,0$

$\mathrm{L}, 1,2$

$\mathrm{K}, 3,0,120,0$

$\mathrm{K}, 4,120,120,0$

L,3,4

LSEL,S,LENGTH „120

LESIZE,ALL,,20

LMESH,ALL

LSEL,ALL

ET,2,LINK8

$\mathrm{R}, 2,1,0.05$

MP,EX,2, 29000000

MP,PRXY, 2, 0.3

TYPE,2

REAL, 2

MAT,2

$\mathrm{K}, 5,120,0,0$

$\mathrm{K}, 6,120,120,0$

L, 5,6

LSEL,S,LENGTH,,120

LESIZE,ALL,,,20

LMESH,ALL

LSEL,ALL

NUMMRG, Node, 0.001

NSEL,R,LOC,X,0

D,ALL,ALL,0

/SOLU

ANTYPE,0

NSEL,ALL

ESEL,ALL

SOLVE

FINISH

/POST1

PLDISP,2

PLNSOL,S,X,0,1.0 


\section{E.3.3 Using Dead and Live Element for column (ANSYS)}

Using dead and live element option with column require defining two load steps as follows:

\section{$\underline{\text { Load Step } 1}$}

In load step 1, two fit-up forces or two fit-up displacements are applied the free ends of the beams. The column is killed or made dead in this load step. In case displacements are applied, reactions developed at displacement application points are the Fit-Up forces. Otherwise the applied forces are the fit-up forces.

\section{$\underline{\text { Load step } 2}$}

In load step 2, the fit-up forces or displacements are removed or made zero followed by making the column alive. At the completion of load step 2 stress in the column and beam can be obtained as shown in Figure E.6.

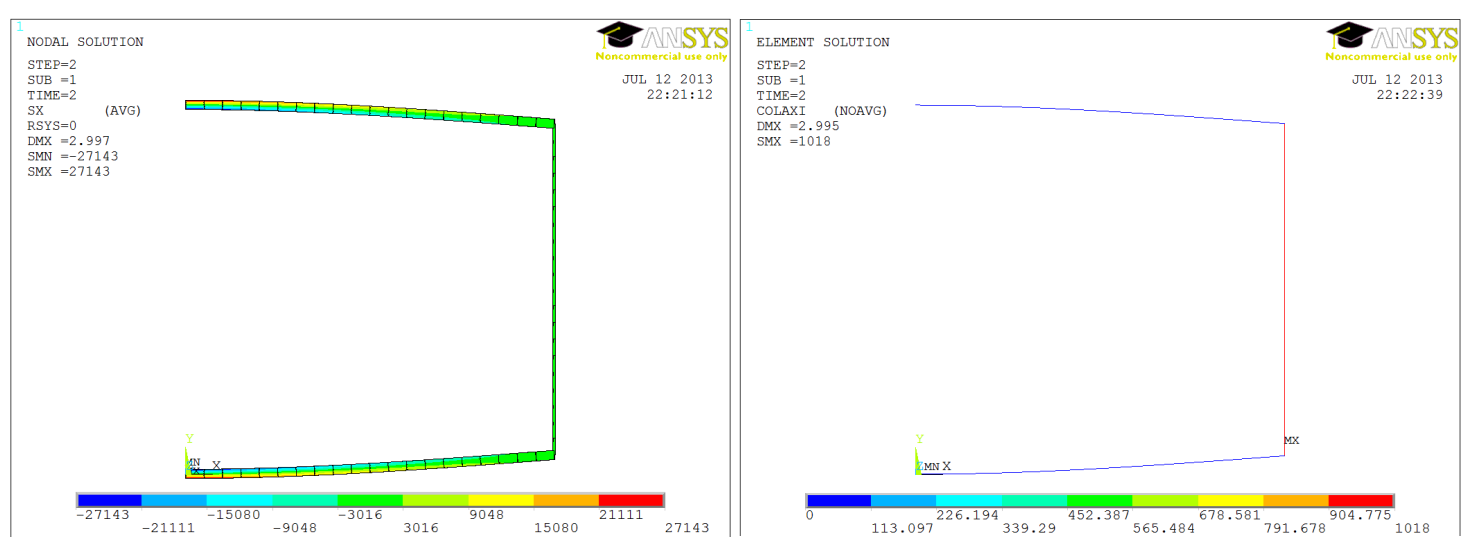

Figure E.6: Stress in the column and beams (psi) at completion of load step of the analysis 


\section{E.3.4 ANSYS code}

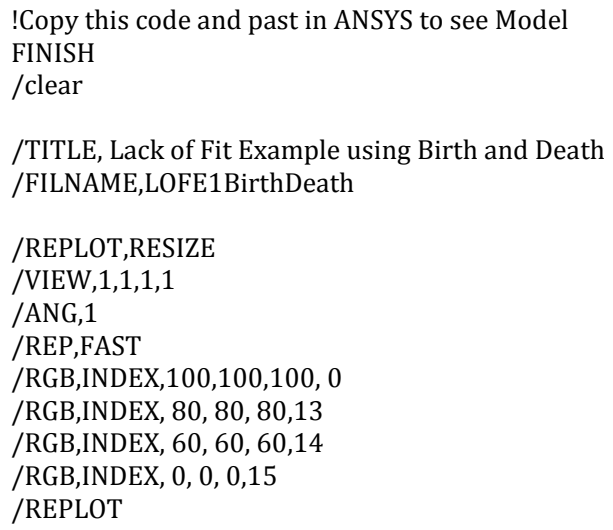

!Meshing

LSEL,S,LOC,Y,120/2

LATT, $2,2,2$

LSEL,ALL 


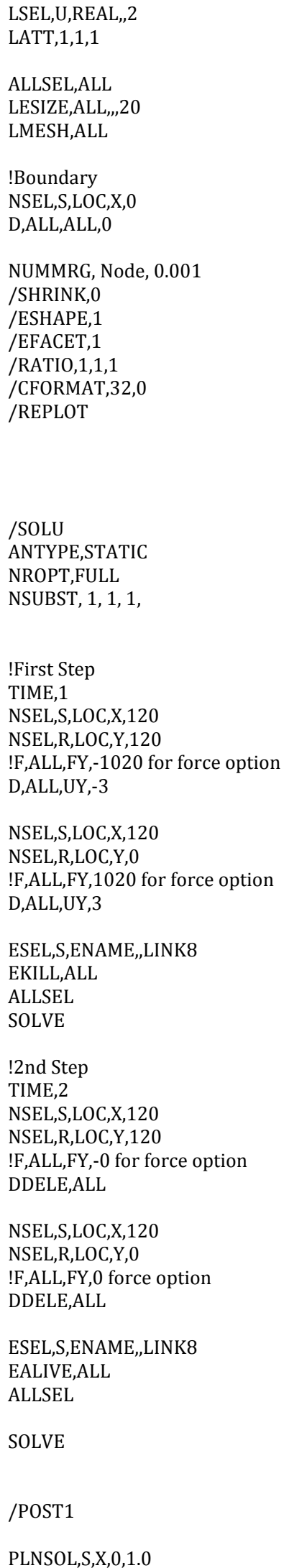

PLNSOL,S,X,0,1.0 
VITA

\section{JAWAD H. GULL}

Born, Multan, Pakistan

2002-2005

B.Sc. Civil Engineering University of Engineering and Technology Lahore Lahore, Pakistan

2006 $3^{\text {rd }}$ position out of 200 students in B.Sc. Civil Engineering

2008-2010

M.Sc. Civil and Environmental Engineering Saitama University

Saitama, Japan

Lecturer

University of Engineering and Technology Lahore

Lahore, Pakistan

2011-2014

Graduate Assistant

Florida International University

Miami, Florida

2012-2014

Doctoral Candidate

Florida International University

Miami, Florida

\section{PUBLICATIONS AND PRESENTATIONS}

Hung, P.V., H., Yamaguchi, Isozaki M., Gull, J. H. 2014. Large amplitude vibrations of long-span transmission lines with bundled conductors in gusty wind. Journal of Wind Engineering and Industrial Aerodynamic 126 pp. 48-59.

Gull, J.H., Azizinamini, A. 2013. Steel Framing Strategies for Highly Skewed Bridges to Reduce/Eliminate Distortion near Skewed Supports. Poster presented at Graduate Research Day, February 22, 2013.

Gull, J.H., Azizinamini, A. 2013. Steel Plate Girder Diaphragm and Cross Bracing Loads. Poster presented at Graduate Research Day, February 22, 2013.

Gull, J.H., Azizinamini, A. 2013. Improved Inspection Techniques for Steel Prestressing/Post Tensioning Stands. Poster presented at Graduate Research Day, February 22, 2013.

Yakel, A., Gull, J.H., Mohammadi, A., Chunn, B., Azizinamini, 2013. A. Managing Florida's Fracture Critical Bridges. Poster presented at Graduate Research Day, February 22, 2013.

Azizinamini, A., Gull, J.H., 2012. Improved Inspection Techniques for Steel Prestressing/Posttensioning Strand. Final Report Volume I, Florida International University, 111 pp. 
Azizinamini, A., Gull, J.H. 2012."FDOT Protocol for Condition Assessment of Steel Strands in Posttensioned Segmental Concrete Bridges." Final Report Volume II, Florida International University, $25 \mathrm{pp}$.

Gull, J.H., Yamaguchi, H., Kumagai, K., Hung, P.V., 2011. Interpretation of field measured Vibrations in transmission lines by gust response analysis. In: Proceedings of $9^{\text {th }}$ International Symposium on Cable Dynamics, Shanghai, China, pp. 281-286.

Gull, J. H. 2010. Interpretation of large field-observed vibrations in transmission line systems based on numerical and measured data analyses. Master Thesis. Saitama University, Saitama, Japan.

Jawad H. Gull, Atorod Azizinamini. 2012. Steel Framing- Straight I-girders with skew. Presentation to Florida Department of Transportation structure design engineers, Sep. 2012, Tallahassee, FL.

Jawad H. Gull, Atorod Azizinamini. 2012. Improved 2D Grid Analysis. Presentation Florida Department of Transportation structure design engineers, Sep. 2012, Tallahassee, FL.

Jawad H. Gull. 2012. Framing of Straight Skewed Steel I-Girder Bridges. Presentation at AASHTO/NSBA Steel Bridge Collaboration Meeting, Nov. 2012, Austin, TX 